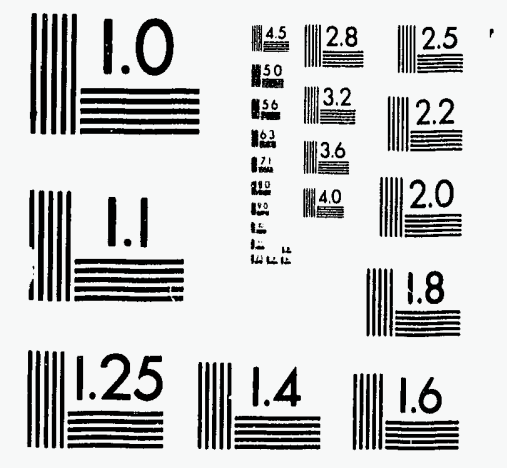



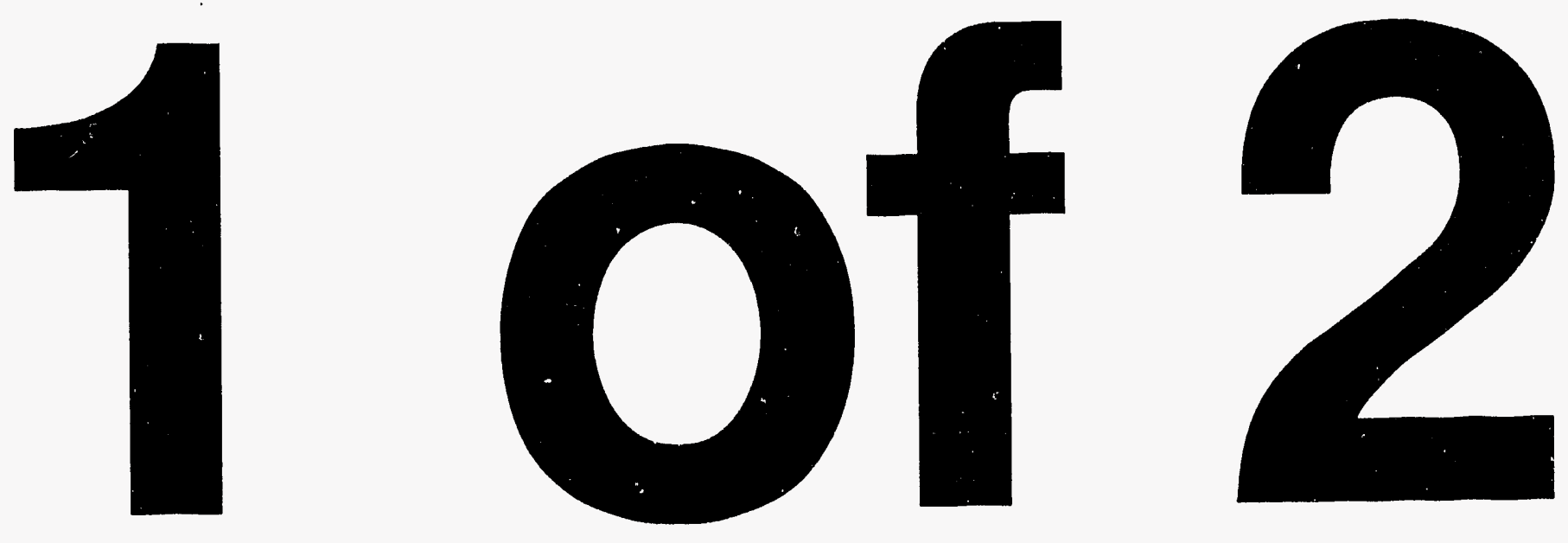

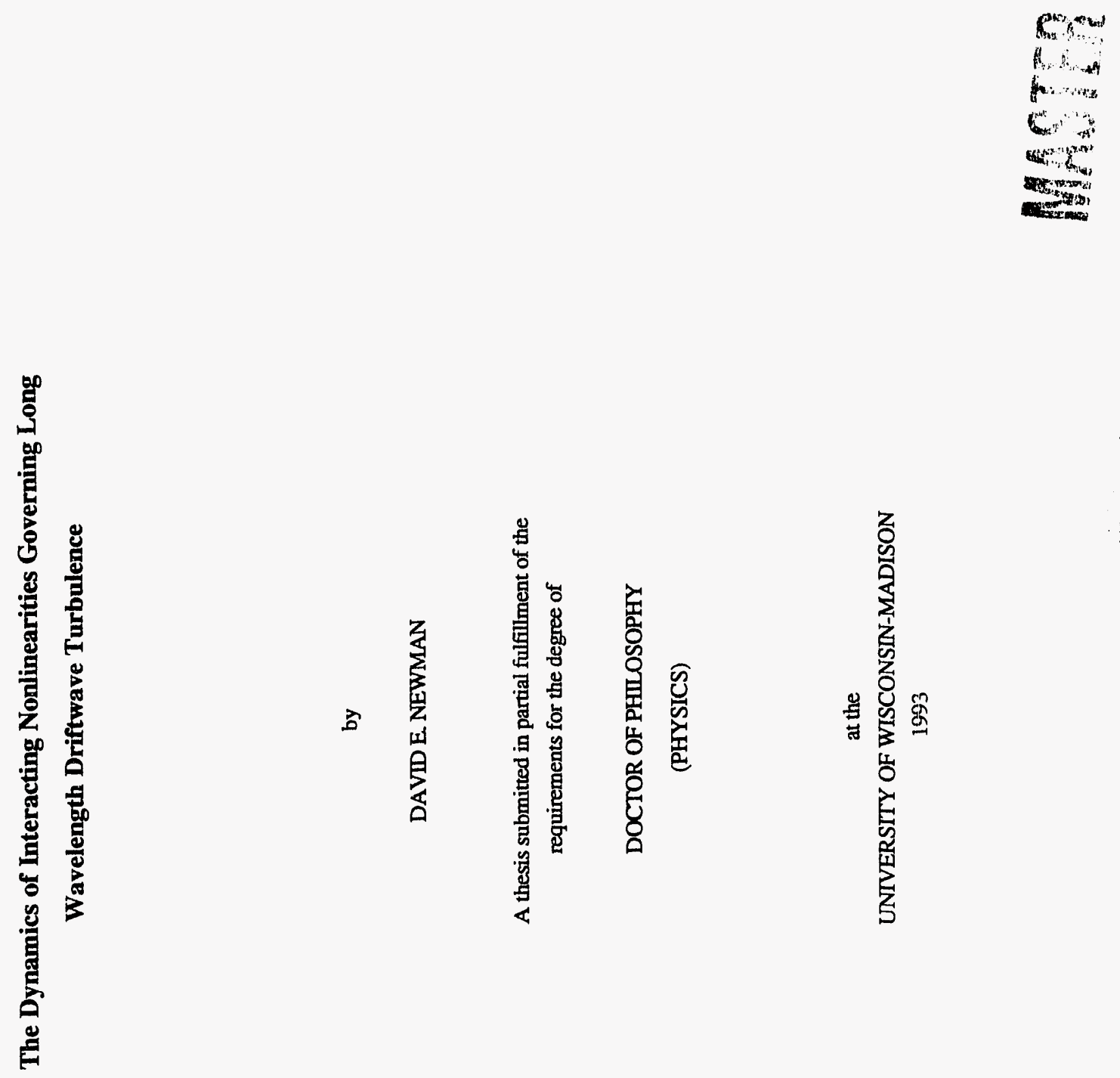

a $\quad 1 \cdots$

(1).

fr.

(4) ?

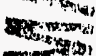

On:

axiremea 
The Dynamics of Interacting Nonlinearities Governing Long

Wavelength Drift Wave Turbulence

\section{David E. Newman}

Under the supervision of Associate Professor Paul W. Terry

at the University of Wisconsin - Madison

\section{Abstract}

Because of the ubiquitous nature of turbulence and the vast array of different systems which have turbulent solutions, the study of turbulence is an area of active research. Much present day understanding of turbulence is rooted in the well established properties of homogeneous Navier-Stokes turbulence, which, due to its relative simplicity, allows for approximate analytic solutions. This work examines a group of turbulent systems with marked differences from Navier-Stokes turbulence, and attempts to quantify some of their properties. This group of systems represents a variety of drift wave fluctuations believed to be of fundamental importance in laboratory fusion devices. From extensive simulation of simple local fluid models of long wavelength drift wave turbulence in tokamaks, a reasonably complete picture of the basic properties of spectral transfer and saturation has emerged. These studies indicate that many conventional notions concerning directions of cascades, locality and isotropy of transfer, frequencies of fluctuations, and stationarity of saturation are not valid for moderate to long wavelengths $\left(\mathrm{k} \rho_{\mathrm{s}} \leq 1\right)$. In particular, spectral energy transfer at long wavelengths is dominated by the $\mathrm{E} \times \mathrm{B}$ nonlinearity, which carries energy to short scale (even in 2-D) in a manner that is highly nonlocal and anisotropic. In marked contrast to the canonical self-similar cascade dynamics of Kolmogorov, energy is efficiently passed between modes separated by the entire spectrum range in a correlation time. At short wavelengths, transfer is dominated by the polarization drift nonlinearity. While the standard dual cascade applies in this subrange, it is found that finite spectrum size can produce cascades that are reverse directed (i.e., energy to high $\mathbf{k}$ ) and are nonconservative in enstrophy and energy similarity ranges (but conservative overall). In regions where both nonlinearities are important, cross-coupling between the nonlinearities gives rise to large nonlinear frequency shifts as well as changes in the spectral dynamics. This profoundly affects the dynamics of saturation by modifying the growth rate and nonlinear transfer rates. These modifications can produce a nonstationary saturated state with large amplitude, long period relaxation oscillations in the energy, spectrum shape, and transport rates. 
I would like to express my deep appreciation to a number of people for their help in getting to this point in my education. First of all I would like to thank my parents Sally and Ted Newman for exposing me to the beauties of science and encouraging me to pursue and explore these wonders. Next I would like to thank my officemates Chris Watts, Jim Hollenberg, Matt Stoneking, Gene Riggs, Brett Chapman, Earl Scime and Eduardo Fernandez with whom I have enjoyed many discussions on physics as well as life. I believe that the setup of our office with a mixture of experimentalists and theorists is ideal and should not be changed. Thanks also go to Andrew Ware for many valuable answers to last minute questions.

The person who deserves the most credit for the incredible amount of fun that I have had doing research over the last few years is Prof. Paul Terry. I truly can not imagine a better advisor nor a better working relationship. I give him my deepest thanks and fear that all future working relationships will pale in comparison

I must also thank Geeta Bhatt for help with the typing of this thesis when, predictably, the time got short. In addition, I could not have made it to this point without my boys, Katmai and Kodiak, quite literally standing by me.

Finally my deepest thanks go to my wife and best friend Uma Bhatt whose support has been invaluable and with whom I have been fortunate enough to enjoy daily valuable scientific discussions in addition to life together.

This work was supported by USDOE grant DE-FG02-89ER53291
Table of Contents

Abstract

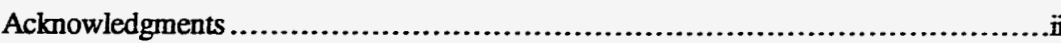

Table of Contents .......................................... iv

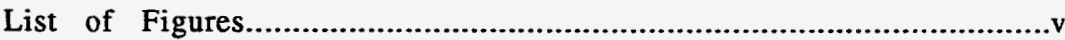

Chapter 1 Introduction......................................................................

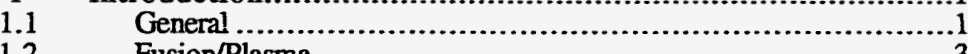

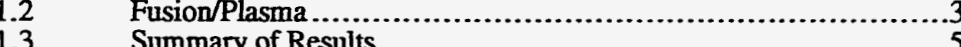

Chapter 2. Dynamics of the ExB Nonlinearity .......................................11 Introduction ........................................................11 Basic Equations and Computational Procedure...................18 Equilibrium and Similarity Range Spectra...........................25 Computional Results............................28

Chapter 3. Improper Spectral flow from Polarization Drift Nonlinearity..................46 Introduction Dimensional Analysis ................................................50 Numerical Analysis ...............................................56 Conclusions and Disclussion .....................................63

Chapter 4. Dynamics of Coupled Nonlinearities .....................................66 66 Basic Model and Properties ... Spectral Transfer ...................................................79 Frequency Spectrum ..........................98

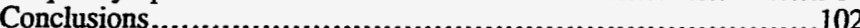

Chapter 5. Multiple Field effects ................................................... Introduction .................................................... 105

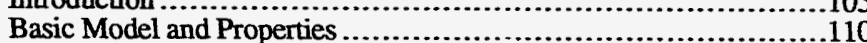
Heuristics of the Cycling .......................................................116 Computational results...............................................118

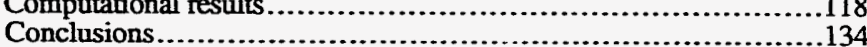

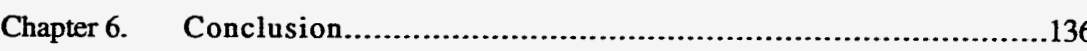

Appendix A Computational Model descriptions........................................141 
List of Figures

Figure

Fig. 2.1. Spectrum of undriven/undamped turbulence at the initial time. The initial phases are random and the initial spectrum fall-off index is $\alpha=-4 \ldots \ldots \ldots \ldots 30$

Fig. 2.2 Spectrum of undriven/undamped turbulence toward the end of the flow regime and just prior to the beginning of the sloshing regime regime and just prior to the beginning of the sloshing regime.
Approximately $5-10$ correlation times have elapsed from the initial

Fig. 2.3 Spectrum of undriven/undamped turbulence in the sloshing regime. The spectrum has been averaged over several correlation times. This spectrum is in the time-asymptotic (relaxed) configuration.

Fig. 2.4 Net energy transfer rates for long and short wavelength bands of constant $k_{y}$ and $k_{x}$. Energy is seen to flow out of the long wavelength bands ( $T_{k}$ negative) and into the short wavelength bands ( $T_{k}$ positive) Distinct flow and sloshing regimes are identifiable

Fig. 2.6 Local and nonlocal transfer rates in the $\mathbf{k}_{\mathbf{y}}$ direction. Transfer is clearly dominated by the nonlocal process, which here is defined as transfer between coupled modes separated by more than half of the wavenumber space.

Fig. 2.7 Local and nonlocal transfer rates in the $k_{x}$ direction. The parity of local and nonlocal transfer rates in the $\mathrm{k}_{\mathrm{x}}$ direction and the disparity of local and nonlocal transfer rates in the $\mathrm{k}_{\mathbf{y}}$ direction indicates a clear anisotropy and nonlocal transfer rates in the $\mathrm{k}_{\mathrm{y}}$ direction indicates a clear anisotropy
in the transfer

Fig. 2.8 Contours of constant density at the initial time in the relaxation of
undriven/undamped turbulence. The peak at low $\mathrm{k}$ in the spectrum ( $\mathrm{Fi}$ undriven/undamped turbulence. The peak at low $\mathrm{k}$ in the spectrum (Fig.
2.1 ) is evident in the large scale structure of the contour plot................37

Fig. 2.9 Contours of constant density in the flow regime. Small scale motion has been excited through the nonlinear transfer process with anisotropy due to the effectiveness of nonlocal transfer in the ky direction. ....................38

Fig. 2.10 Contours of constant density in the sloshing regime (see Fig. 2.3) Sloshing has isotropized the contours.

Fig. 2.11 Spectrum of turbuience driven at long wavelength and damped by a hyperviscosity at short wavelength with an inertial range in the
Fig. 3.1 The magnitude of the normalized seif-similar energy flows in the proper and reverse directions as a function of $\mathrm{l}=\mathrm{kinj} / \mathrm{kmax}$ Normalization is with respect to the total energy carried in seif-similar flows.

Fig. 3.2 The magnitude of the normalized self-similar enstrophy fluws in the proper and reverse directions as a function of $I=k i n j / k m a x$. proper and reverse directions as a function of $1=k$ nin kmax. Normalization is with respect to the total enstrophy carried in self-similar
flows...

Fig. 3.3 Energy transfer rate for bands with $k_{y}=$ constant in numerical solutions of Hasegawa-Mima turbulence. Transfer is from a large perturbative pulse (at $\mathrm{k}_{\mathrm{inj}}=7$ ) applied to a quasi-equilibrium spectrum.

Fig. 3.4 Enstrophy transfer rate for the same case as Fig. 3.3.

Fig. 4.1 Equilibrium spectrum of undriven/undamped turbulence with the only polarization drift nonlinearity. This spectrum is the relaxed state that has evolved from an initial spectrum with a fall-off index of $a=-4$. ............82

Fig. 4.2 Time averaged energy transfer rate during the relaxation leading to the spectrum of Fig. 4.1. The error bars indicate the standard deviation from the mean value plotted in the figure ....................................

Fig. 4.3 Local and nonlocal energy transfer rates from the band with negative transfer (energy outflow) during the relaxation leading to the spectrum of

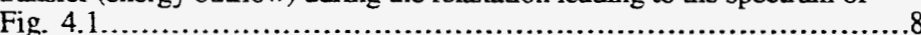

Fig. 4.4 Spectrum of undriven/undamped turbulence with both the $E \times B$ and polarization drift nonlinearities ( $\mathrm{E} \times \mathrm{B}$ nonlinearity dominates weakly). This s the spectrum that occurs several eddy turnover times after the

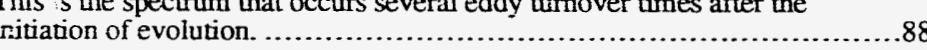

Fig. 4.5 Final (time asymptotic) spectrum for undriven/undamped turbulence with both the $\mathrm{E} \times \mathrm{B}$ and polarization drift nonlinearities $(\mathrm{E} \times \mathrm{B}$ nonlinearity dominates weakly).

Fig. 4.6 Time evolution of the enstrophy for four spectrum configurations..............90

Fig. 4.7 Spectrum of turbulence driven at long wavelength and damped by a hyperviscosity at shor wavelength with an inertial range in the intermediate modes.

Fig. 4.8 Evolution of the spectrum with driving and damping (under the $E \times B$ nonlinearity only) subsequent to a impulse of energy localized to rectangular annulus in $k$ space.

Fig. 4.9 Evolution of the spectrum with driving and damping (under the polarization drift nonlinearity only) subsequent to a impulse of energy ocalized to a rectangular annulus in $\mathrm{k}$ space. ...............................95 
Fig. 4.10 Evolution of the spectrum with driving and damping and both nonlinearities subsequent to a impulse of energy localized to a rectangula annulus at low $k$ in the $E \times B$ subrange.

Fig. 4.11 Evolution of the spectrum with driving and damping and both nonlinearities subsequent to a impulse of energy localized to a rectangula annulus at high $\mathrm{k}$ in the polarization drift subrange.

Fig. 4.12 Frequency spectra of a mode with small wavenumber during relaxation without driving or damping. The spectra correspond to a) $\mathrm{E} \times \mathrm{B}$ nonlinearity only, b) polarization drift nonlinearity only, c) both nonlinearities

Fig. 4.13 Frequency spectra of a mode with large wavenumber during relaxation without driving or damping. The spectra correspond to a) $E \times B$ nonlinearity only, b) polarization drift nonlinearity only, c) both

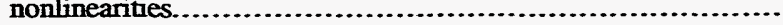

Fig. 5.1 The real part of the dispersion relation.

Fig. 5.2 The imaginary part of the dispersion relation

Fig. 5.3 During a cycling event the total energy is seen to fluctuate by more than $15 \%$. The period is 5-10 "eddy turnover times" as defined by a mode fluctuation time.

Fig. 5.4 The system with the large amplitude cycles has the longer more regular period.

Fig. 5.5 The flux from the 2 more active modes "bursts" coincident with the fluctuation. The burst in the most active mode leads the fluctuation by a small phase.

Fig. 5.6 The total energy evolution with the $\mathrm{E} \times \mathrm{B}$ flow evolution during the fluctuations. The EXB transfer goes to zero at the end of a cycle then begins again.

Fig. 5.7 Note the mean frequency up-shift in the peak phase of a cycle. This would tend to decrease the growth rate relative to the trough phase..................134

Fig. 58 This $\mathrm{k}$ spectrum shows the energy dominantly in the middle $\mathrm{k}$ modes.

This spectrum is at the begining of a cycle.

Fig. 5.9 The $\mathrm{k}$ spectrum is in the middle of a cycle with energy moving from the early dominant modes to lower $\mathrm{k}_{\mathrm{y}}$ modes.

Fig. 5.10 This $k$ spectrum is at the end of a cycle, showing the energy being transfered out to high $\mathrm{k}_{\mathrm{x}}$ with some spreading in $\mathrm{k}_{\mathrm{y}}$
Fig. 5.11 Real space isodensity contours early in a cycle. The structures are relatively isotropic and homogeneous.

Fig. 5.12 Real space isodensity contours midway through a cycle showing elongation in the $\mathrm{x}$ direction.

Fig. 5.13 Isocontours at the end of a cycles. The structures are seen to be breaking up...........................................133 


\section{Chapter 1}

Introduction

\subsection{General}

The study of turbulence is primarily the study of the dynamics of the nonlinearities governing the turbulence. This area of study has a long and distinguished kistory spanning over one hundred years of experimentation, analysis and modeling. Despiie successes based on the scaling analysis begun with Reynolds and continued by Kolmogorov ${ }^{1}$ and others: on analytic closures, physical modeling, computation, and detailed observation, there is still much in turbulence that remains a mystery. Studying the dynamics of multiple turbulent nonlinearities, their interplay with each other and with sources and sinks in their domain, yields a wide variety of fascinating results. Some results and methods of approaching problems are found to be inexplicably general in their applicability while other accepted properties of turbulcist systems are found to be limited in their validity.

As a starting place in investigating turbulence it is valuable to use as simple a system as possible in order to fully understand the dynamics without additional complications. One step in this simplification is the use of 2 dimensional models. The validity of such models tends to be much greater than one might expect in a 3 dimensional world. Many situations exist which can cause the reduction of a system from 3-D to 2-D2. In neutral fluids, rotation causes a separation of 2 and $3 \mathrm{D}$ dynamics for imbedded turbulence with the correct spatial dimensions and energy. This allows much of the large scale ocean and atmospheric dynamics to be well modeled by 2-D quasi-geostropic models ${ }^{3}$. Stratification can also cause a division of the two types of dynamics with all turbulent fluctuations having a characteristic scale length (le) greater than the stratification scale $\eta_{S}$ ) being governed by 2-D dyramics within the plane of stratification. Similarly, most surface physics is explicitly 2-D and as such is governed by 2-D dynamics. The introduction of a magnetic field makes the dynamics of charged particles along the field intrinsically different from the dynamics perpendicular to the field. This often allows the perpendicular dynamics to be treated as 2-D with little loss of precision. The research described here is primarily focused on plasma turbulence whose 2 dimensional nature is due to the magnetic field which is perpendicular to the plane of the dynamics. Nevertheless much of the insight gained from these studies should be applicable to other (non-plasma) systems.

In addition to serving as a paradigm for a variety of turbulent systems, the models described here strongly suggest the existence of a class of interesting dynamics in many systems that may often be overlooked. This class of dynamics comes from the direct interaction between multiple nonlinearities. A common feature of turbulent systems is the existence of multiple nonlinearities of differing order. The ordering could be based on amplitude, as in a quadratic nonlinearity vs. a cubic nonlinearity, or on scales, as in the nonlinearity having three derivatives vs. one having four derivatives, etc. In general the system is simplified by ignoring the subdominant nonlinearity. While this may be valid in some cases, and is usually a reasonable first step, it may also be ignoring important dynamics in the cross-coupling. These cross-coupling dynamics may not be as subdominant as the dynamics of the neglected nonlinearity and as such may be important to the overall system dynamics.

One of the most cherished concepts in turbulence research is the inertial range. This is a region in $\mathbf{k}$-space where the nonlinear term ( $\mathbf{v} \cdot \nabla \mathbf{v}$ for Navier-stokes turbulence) is much larger then any linear terms ( $v \nabla^{2} v$ for Navier-stokes). Using the existence of such a region the cascade dynamics can be studied both analytically and computationally ${ }^{4}$. Much of the analytic work also assumes that the sources and sinks of energy (i.e., the stirring and viscous damping) are separated by an inertial range of infinite extent. While these concepts are useful for the purpose of getting results, they can sometimes be misleading. In general 
if an inertial range exists it is limited in extent and has sources and sinks arranged in a fashion that is less than optimal from the perspective of a theorist. In any physically realistic model the energy will go from the source to the sink irrespective of the preferred dynamics of the nonlinearity governing the cascade. For example a system can be constructed with the characteristic that the nonlinearity prefers to transfer energy to high $\mathrm{k}$ from low $\mathrm{k}$. This system can also be arranged so the source is at high $\mathrm{k}$ and the sink is at low $\mathbf{k}$. If the system is physically realistic a steep gradient in energy will be set up from source to sink with much more energy in the high $\mathbf{k}$ driven modes than in the low $\mathbf{k}$ damped modes. The system will, however, still saturate by transferring the energy down the gradient to the sink. The saturation level is likely to be higher then in the rpposite case with driving at low $\mathbf{k}$, damping at high $\mathbf{k}$ and a preferred nonlinear transfer direction being to high $\mathbf{k}$.

In many systems, including most turbulent plasma models, in addition to the relatively small region (in $\mathbf{k}$ space) in which the model is valid, the sources and sinks are distributed. This leads to a small or non-existent true inertial range, adding another level of complexity in the path of understanding the dynamics of the system.

\subsection{Fusion/Plasma}

In the pursuit of fusion as a viable source of energy a number of hurdles must be crossed. One of the most serious of these is the problem of confinement of energy. In order for a self-sustaining nuclear fusion reaction to occur the triple product of density (n), temperature $(T)$, and energy confinement time $(\tau), \mathrm{nT} \tau$, must be sufficiently large 5 . This means the density must get large enough, the temperature must get high enough, and the energy confinement time must be long enough for an acceptable value of the triple product. Preventing the extension of the confinement time are a number of processes which can be roughly broken down by scale size. At the smallest size scale is classical molecular (or atomic) diffusion. Classical diffusion is a very slow process even at the densities and temperature in the atmosphere. In a typical fusion device such processes would not have a great effect on the confinement time. At the large scale end of the spectrum are global disruptions. These are usually catastrophic magnetic events which essentially dump all or most of the energy onto the containment shell in a very short amount of time. At the time of a disruption $\tau$ goes to near zero implying no confinement and complete suppression of any fusion reactions. As $\mathrm{n}$ and $\mathrm{T}$ get larger the stored energy increases and these major disruptions become increasingly serious due to the potential for damage to the shell. Fortunately, regimes of operation have been found in which these disruptions do not seem to occur, thus, removing the problem. This leaves the intermediate scales as the source of the anomalous transport which degrades the confinement 6 . This transport probably does not have the same source everywhere in the device. At some radial locations the transport maybe due to electrostatic fluctuations while at other places it may be due to interacting magnetic islands 7 . Which of the processes is the limiting one is not yet clear and it may be that they all work in concert. Nevertheless it does seem clear that fluctuation driven turbulent transport is important in the system and must be understood.

Large scale (long wavelength) turbulence is an area of intense current research from both the experimental and theoretical perspectives. This research has beea particularly focused on the core fluctuations as these fluctuations are thought to be responsible for the transport of energy from the hot core to the outer regions of the plasma. One of the models considered as a viable candidate for the core fluctuations is the dissipative trapped ion convective cell turbulence (DTICC) model. In the late $70^{\text {'s }} \mathrm{s}$ this model was considered ${ }^{8}$ and rejected when the catastrophic Bohm-like transport that had been predicted was not realized when successful operation of the Princeton Large Torus in the 4 kev range was achieved. More recent theoretical work ${ }^{9}$ suggests that this mode should not give catastrophic transport and is therefore a valid candidate for core fluctuations. Much recent experimental work including beam emission spectroscopy ${ }^{10}$ and correlation reflectometry ${ }^{11}$ give an indication that long wavelength fluctuations do exist in the core and may be important to transport. 
In addition to being useful as a possible candidate to explain core fluctuations, the DTICC model is useful as a starting paradigm for drift wave turbulence. Within drift wave turbulence models two of the most prevalent nonlinearities, and therefore two of the most important, are the EXB and polarization drift nonlinearities. Because DTICC turbulence is easily represented by a 2-D one-field fluid model (Kadomtsev-Pogutse ${ }^{12}$ ), it readily allows the exploration of the dynamics of the ExB nonlinearity. With straightforward extension the polarization drift nonlinearity (like that in the Hasagawa-Mima equation ${ }^{13}$ ) can be added. This extends the model to be generic enough in its nonlinear behavior so as to cover most drift wave turbulence. As an example, the Terry-Horton equation ${ }^{14}$ has both the EXB and the polarization drift nonlinearities in a one-field model for trapped electron and universal modes. These models easily allow the investigation of the different aspects of these nonlinearities and their interactions. Furthermore they may provide insight into the behavior of turbulent nonlinearities in general

\subsection{Summary of Results}

This work consists of a series of studies concentrating on understanding the dynamics of two of the nonlinearities which govern drift wave turbulence. With a progression of models each nonlinearity is investigated individually then they are combined to allow the study of interacting nonlinearities. Finally the models are extended to two fields to allow the interplay between the nonlinearities to feed back on the system. This allow the cross-coupling to have a direct effect on the linear growth and transport. The four models are each realiy just an extension of the previous ones. The first is a one-field Dissipative Trapped Ion Convective Cell (DTICC) model with just the EXB nonlinearity. Next is a model used to study the polarization drift nonlinearity (or the Navier-Stokes nonlinearity). This model is the one-field Hasagawa - Mima (H-M) model. The model combining the two nonlinearities is the Dissipative Trapped Electron mode (DTEM1) model. This is then extended to a iwo-field Dissipative Trapped Electron mode (DTEM2) model to allow the self-consistent feedback. In all of these models a variety of diagnostics are used in a range of different simulations to probe the dynamics of the systems. The diagnostics used include time histories of total energy, flux, and enstrophy, time histories of individual mode energy enstrophy, and flux as well as frequency and $\mathbf{k}$ spectrum evolutions. A diagnostic was developed to track the nonlinear transfer of energy and enstrophy between modes (or bands of modes). This has proved to be invaluable in deciphering the dynamics over the full range of $\mathbf{k}$ space. In the different models a sequence of types of simulations are performed. Some simulations are nun with no driving or damping to allow the nonlinearities to relax the system, thereby simulating pure inertial range transfer. Other cases have driven/damped systems run to saturation in which some set of modes are undriven and undamped and which therefore comprise an inertial range. In at other set of cases the driven/damped system is allowed to reach saturation at which time a localized energy pulse is inserted. The pulse is allowed to relax and the relaxation is followed to discern the dynamics involved. Finally, scans of parameter space are performed in order to investigate any anomalous behavior. A summary of the main results follows.

The basis for the Kolmogorov spectrum is the assumption that inertial ranges are dominated by a local self-similar transfer or cascade. Using a combination of the Kolmogorov spectrum and equilibrium statistical mechanics it is possible to infer the preferred direction of flow for a given nonlinearity. The dynamics of most turbulent systems are governed by nonlinearities which are themselves isotropic so in the absence of anisotropic driving, damping or geometry the turbulence is also expected to be isotropic. The DTICC model is used to study the dynamics of an anisotropic nonstandard nonlinearity. It is found that this nonlinearity transfers energy nonlocally from low ky to high ky. This leads to a saturated spectrum much flatter in the ky direction than that predicted by 
Kolmogorov theory. This should not be surprising as the lack of a local transfer induced similarity range precludes the use of Kolmogorov theory.

Going hand in hand with the concept of local transfer in an inertial range is the idea of conservative cascades. The nonlinearity of the DTICC $n$,del (EXB nonlinearity), $\nabla$ $(\partial \mathrm{n} / \partial \mathrm{y}) \times \mathrm{z} \cdot \nabla \mathrm{n}$, has only cne quadratic invariant (energy) and consequently has only a direct cascade. The H-M model is used to study the dynamics of the polarization drift nonlinearity ( $\nabla \phi \times z \cdot \nabla \nabla{ }^{2}$ ) which has 2 quadratic invariants, energy and enstrophy. These 2 invariants give rise to the same dual cascade that is familiar from 2-D Navier-Stokes turbulence. In this cascade energy cascades conservatively to low $\mathbf{k}$ and enstrophy cascades conservatively to high $\mathbf{k}$. The early work of Kraichnan (1964) on these cascades used the infinite extent of the cascade to show that energy is the only quantity cascaded in the low $k$ region and enstrophy is the only quantity cascaded in the high $\mathrm{k}$ region. It is found here that if the spectrum is not infinite in extent there is some "improper" transfer. In the energy transfer range some enstrophy is transferred and in the enstrophy transfer range some energy is transferred. The amount of "improper" transfer depends on both the extent of the inerial range and the position, within that range, of the source of free energy (the injection range). It is quite possible to arrange a situation in which more energy or enstrophy flows in the improper direction then in the proper direction. This is important to keep in mind when inferring dynamical properties from the conservation laws of the system.

Nonlinearities are by definition not additive, yet it is assumed that it 2 nonlinearities have different regions of dominance in $\mathrm{k}$ spule, in the region dominated by one nonlinearity the other nonlinearity can be ignored. The DTEM1 model is used to investigate the effects of two nonlinearities interacting in one system. It is found that at the extremes in which one dominates the other by more then an order of magnitude, it is justifiable to drop the subdominant nonlinearity. However in the often large region where the relative strength of the nonlinearities are within an order of magnitude of each other, new dynamics occur which are not explainable by a linear super-position of the 2 nonlinearities. The "cross-coupling" dynamics are qualitatively different and include both a change in the transfer dynamics and a nonlinear frequency shift. These cross-couplirg dynamics occur dominantly in the "crossover" region in which the two nonlinearities are of comparable magnitude. The frequency shift can be as large as a few times $\omega^{*}$ and is found to be proportional to ky. The shift observed from simulation qualitatively agrees with the shift predicted by closure theory. Such shifts could help explain frequencies observed in computation which seem anomalously shifted away from the frequency expected from linear dispersion relations. The influence of the crossover region creates 5 effective regimes: 1 ) at the low $k$ extreme, transfer dynamics are governed solely by the $\mathrm{E} \times \mathrm{B}$ nonlinearity; 2) at $\mathrm{k}$ values between the crossover region and the region of $\mathrm{E} \times \mathrm{B}$ dominance both the $\mathrm{E} \times \mathrm{B}$ and the cross-coupling dynamics are important; 3 ) in the crossover region, the characteristic dynamics are dominated by the interaction of the two nonlinearities, these dynamics are largely independent of the EXB and polarization drift dynamics; 4) in the region above the crossover region but below the polarization drift dominated region, both the cross-coupling and the polarization drift dynamics are important; and 5) at the highest $k$ values the polarization drift dynamics dominate. Before ignoring a subdominant nonlinearity it may be important to assure oneself that the nonlinearity of interest is sufficiently dominant so as to dominate the cross-coupling dynamics as well as the subdominant nonlinearity.

In order to self-consistently include the feedback effect of the frequency shift on linear growth and transport fluxes, it is necessary to extend the model beyond a one-field model to a two-field model. The DTEM2 model is used to study these interaction feedbacks. The most striking result from the two-field studies is the lack of a stationary saturated state. As with most driven/damped turbulent systems this system does reach a saturated state dependent on the various parameters. These parameters include growth, damping and the ratio of $E \times B$ to polarization drift nonlinearity strength but, for fully 
developed turbulence, do not include initial conditions. In this system the saturated state is found to be non-stationary, instead exhibiting oscillations (cycles) with amplitude of order $50 \%$ and oscillation period on the order of $10 \tau_{\mathrm{e}}$ (eddy turnover times). During these cycles most of the relevant quantities also undergo large fluctuations. These include transfer rates and flux as well as frequency and $\mathrm{k}$ spectra. These cycles may be understood in terms of a simple heuristic relaxation oscillation model. It is suggestive of an intriguing pessibility that this type of oscillation may in fact exist in experiment , but may effectively be "washed out" by averaging over a few cycles in the measurement process. Since these models are all local, it is also possible that spatial measurmental averaging could wash out the fluctuations. If they do exist, it might suggest that transport is a much more locally intermittent event then generally believed.

The organization of the thesis is as follows: chapter 2 contains work on the Dissipative Trapped Ion Convective Cell turbulence model. This work focuses on the novel features of the EXB nonlinearity. Chapter 3 contains analytical and computational results dealing with the effect of finite spectrum size on the polarization drift nonlinearity. This is presented in the context of a Hasagawa - Mima type model which is nearly isomorphic to the Navier - Stokes equation. Chapter 4 deals with the one - field Dissipative Trapped Electron mode turbulence model. This model is used to investigate the interaction between the two nonlinearities $(\mathrm{E} \times \mathrm{B}$ and polarization drift). In chapter 5 the dissipative trapped electron mode model is extended to two fields in order to explore the feedback of the nonlinear cross-coupling. Brief conclusions are presented in chapter 6 followed by Appendix A which contains some information on the numerics and a commented listing of the main part of the two-field codes. Finally, in Appendix B some calculations are presented on extending Kolmogorov analysis to nonlocal (non self-similar) transfer. It should be noted that each of these chapters is fairly self contained with introductions which elaborate on the brief exposition of concepts given in this introduction.

\section{References}

A. N. Kolmogorov, J. Fluid Mech., 12. pp 82-85 (1962)

M. Lesieur. "Turbulence in Fluids", (Kluwer Academic Publishers, Boston, 1990)

J. Pedlosky, "Geophysical Fluid Dynamics", (Springer Verlag, 1979,

R.H. Kraichnan, Phys. Fluids 10, 1417 (1967)

J. Hugill, Nucl. Fusion 25, 331 (1983)

J.D. Callen, Phys. Fluids B 2, 2869 (1990).

C.C. Hegna and J.D. Callen, Phys. Fluids B 4, 1855 (1992).

R. Saison, H.K. Wimmel, and F. Sardei, Plasma Physics 20, 1 (1978)

P.H. Diamond and H. Biglari, Phys. Rev. Lett. 65, 2865 (1990)

10 R.J. Fonck, S.F. Paul, D.R. Roberts, Y.J. Kim, N. Bretz, D. Johnson, R. Nazikian and $G$ Taylor, 18th European Conference of Controlled Fusion and Plasma Physics (European Physical Society, Vol. 15C, Part I, 1991) p. I-269.

11 P. Cripwell and A.E. Costley, 18th European Conference of Controlled Fusion and

Plasra Physics (European Physical Society, Vol. 15C, Part I 1991) I-17.

12 B.B. Kadomtsev and O.P. Pogutse, Reviews of Plasma Physics Vol 5, edited by

M. Leontovich (Consultants Bureou, New York, 1970), p. 387.

13 A. Hasegawa and K. Mima. Phys. Rev. Lett. 39, 205 (1977).

14 P.W. Terry and W. Horton, Phys. Fluids 25, 491 (1982). 


\section{Chapter 2 \\ Dynamics of the $\mathbb{E} \times \mathbf{B}$ Nonlinearity}

\subsection{Introduction}

The possibility that turbulence driven by unstable trapped ion modes plays a role in core fluctuations and transport in tokamaks has generally been discounted throughout the past decade. At one time however, trapped ion modes were thought to represent a potentially serious confinement problem for auxiliary heated (multi-kilovolt) plasmas 1 . Indeed, catastrophic Bohm-iike transport was predicted. This prediction was premised on a presumed inverse cascade of energy from the already long wavelengths of the unstable fiuctuations, and the fact that fluctuation levels would be large, given the long radial correlation length of the turbulence. The successful operation of the Princeton Large Torus device at ion temperatures in excess of $4 \mathrm{Kev}$ without catastrophic confinement problems was generally taken as an indication that trapped ion mode activity was somehow absent from hot auxiliary heated tokamak discharges. Excepting some early efforts to explain this seeming lack of trapped ion mode turbulence ${ }^{2}$ and some work on low collisionality ion temperature gradient driven turbulence ${ }^{3}$, trapped ion turbulence has generally been ignored.

Recent work on trapped ion mode turbulence based on approximate analytic solution of renormalized Kadomtsev-Pogutse fluid equations ${ }^{4}$ contends that these fluctuations can not be discounted as an important component of core turbulence and may in fact comprise the low frequency large amplitude extreme of experimental spectra. Contrary to the predictions of Bohm-like transport ${ }^{1}$, this work asserts that trapped ion convective cell urbulence drives transport which is not excessively large, but is comparable in magnitude to the transport produced by trapped electron turbulence. This contention is supported by two facts. The first is that the turbulent radial flow associated with trapped ion convective cell turbulence is small, offsetting the large fluctuation level in the quadratic moments which determine the transport fluxes. The second is the prediction that spectral energy transfer is not characterized by an inverse cascade, but rather is directed to short wavelengths. The latter precludes catastrophic condensation of energy at the largest scales of the system.

There is mounting experimental evidence that fluctuations with large radial correlation length are present in the core of tokamak plasmas. Observed spectra from scattering diagnostics have long exhibited an increase of spectral energy toward the smallest resolved wavenumbers, typically with no turnover evident over the range of wavenumbers for which measurement is possible. Recently, new fluctuation diagnostics with the capability of providing spatially resolved local measurements of core turbulence have been developed. Both beam emission spectroscupy 5 and correlation reflectometry 6 find evidence (the former in TFTR, and the latter in JET) for fluctuations inside r/a of 0.7 with radial correlation lengths of several centimeters. In both cases, the frequency of the fluctuations is very low or nearly zero, once the rotation induced Doppler shifts are subtracted. Intriguing links with global confinement are evident. While it has not been possible to associate these fluctuations with any given model, trapped ion convective cell turbulence is clearly a candidate.

This chapter describes a numerical study of dissipative trapped ion convective cell turbulence. Motivated by the issue of the energy transfer direction in wavenumber space for trapped ion turbulence, key facets of the spectral energy transfer process are examined. In order to isolate basic physical processes and enable comparison with analytic theory, a simple 2-D single field model is utilized. This model is based on the fluid responses for trapped ions and electrons first used by Kadomtsev and Pogutse. The link between electrons and ions provided by quasineutrality enables a single field description. Rapid trapped particle bounce motion, re sricting the development of parallel dynamics, provides 
the rationale for a two dimensional treatment. The model incorporates the ExB nonlinearity, the dominant nonlinear transfer mechanism for long wavelength fluid plasma turbulence Consequently, the turbulent transfer properties of dissipative trapped ion convective cell turbulence, as described by the model, apply to a broader class of long wavelength fluctuations, including those resulting from trapped electron modes.

In addition to determining the direction of energy transfer in wavenumber space, an investigation of other properties relating to the spectral transfer process is described in detail. This includes the degree to which transfer in wavenumber space is local, as implicit in Kolmogorov-type similarity arguments which envision an energy transfer through all scales at precisely the same rate, or nonlocal, and therefore at variance with the standard view of cascades. The degree of isotropy or anisotropy in the energy transfer process and in the spectrum itself is also investigated. Finally, the direction of energy transfer is examined in relation to the quadratic invariants of the nonlinearity and the equilibrium spectrum. The latter ties in with statistical mechanics calculations frequently used to infer the direction of cascades 7,8 . Clearly, these properties impact the spectrum, the turbulence level, and the magnitude of spatial transport. At a more fundamental level, they affect the basic characterization of turbulence and turbulent cascades.

Spectral transfer and its characterization in terms of cascades has long been a central part of the conceptualization of turbulence. In Navier-Stokes turbulence, for example, it is well established that the energy transfer can be represented by a self-similar cascade process. In three dimensions, this process conservatively transfers energy to small scales. In two dimensions, the invariance of an additional quantity, enstrophy, or mean square vorticity, precludes the self-similar transfer of energy to small scale. It is possible to conserve both quantities, however, if enstrophy is transferred to small scale and energy to large scale. By analogy with 2-D Navier-Stokes turbulence, it might be inferred (mistakenly, as will become apparent) that dissipative trapped ion convective cell turbulence undergoes an inverse energy cascade (cascade to long wavelength). However, it is the simultaneous invariance of energy and enstrophy which directly underlies the dual cascade of 2-D Navier-Stokes turbulence and not the number of dimensions (except through the number of invariants).

It is often possible to infer the direction of spectral transfer from closure equations. These describe the average transfer consistent with the statistical ansatz invoked to obtain the closure. The statistical hypotheses upon which closures are predicated are, in general, very difficult to validate and are knowa to be violated by fluctuations which are spatially intermittent. Furthermore, a number of other approximations and simplifications typically enter into analytical results obtained from closure equations. In particular, the closure is most often applied to one-point equations. While one-point analyses simplify the determination of a saturation level, they neglect the incoherent transfer process required for energy conservation. For these reasons, other methods for inferring the spectral transfer have been developed.

The most widely used method is based on equilibrium statistical mechanics ${ }^{7,8}$. This method is appealing for its direct use of the dynamical invariants in obtaining equilibrium spectra of the invariant quantities. Its weakness lies in the somewhat tenuous connection between equilibrium quantities and the properties of turbulence, which generally are far from equilibrium. At the minimum, the use of an equilibrium spectrum to infer a spectral transfer direction requires a knowledge of the steady state spectrum set up under forcing and dissipation, and the assumption that nonlinear transfer in the steady state is in the direction which would tend to drive the spectrum towards its equilibrium configuration. An additional weakness arises from the possibility that additional invariants exist which constrain the transfer but are not among those known and included in the calculation of the equilibrium spectrum, thereby compromising the equilibrium prediction. Not withstanding these difficulties, equilibrium statistical mechanics conrectly predicts the direction of spectral 
transfer in 2-D and 3-D Navier-Stokes turbulence ${ }^{7,8}$, and has been used in many other types of turbulence ${ }^{9-10}$.

In the present work, nonlinear transfer in the numerical simulation of the Kadomtsev-Pogutse fluid model is directly measured in distinct regions of wavenumber space. In order to eliminate the transfer imposed by any particular wavenumber space distribution of sources and sinks in favor of the conservative transfer produced by the nonlinearity, transfer is determined for undriven/undamped turbulence starting from a finite amplitude initial state with a given spectrum. These results establish the direction of inertial transfer of energy in a steady state whose spectrum is similar to the spectrum chosen as an initial condition. The initial spectrum relaxes under the inertial transfer, producing timeasymptotically an equilibrium spectrum which can be compared with the spectrum predicted by equilibrium statistical mechanics on the basis of the known dynamical invariants. These studies therefore provide a test of the validity of the methodology of equilibrium statistical mechanics for predicting spectral transfer directions, as well as a check on the predictions of the closure theory4.

The concept of a wavenumber cascade is usually thought of as a local process whereby energy is passed between scales which are adjacent in wavenumber space. The renormalized Kadomtsev-Pogutse equation provides for both local and nonlocal transfer of internal energy $\left.\left|\tilde{n}_{k}\right|\right|_{\text {in }}$ wavenumber space. According to the renormalized equation,

$\frac{1}{2} \frac{\partial\left|\tilde{n}_{k}\right|^{2}}{\partial t}-\gamma_{k}\left|\tilde{n}_{k}\right|^{2}+T_{k}=0$

the evolution of energy in the mode $\mathrm{k}$ is governed, apart from linear driving and damping $\left(\gamma_{k}\right)$, by a transfer rate $T_{k}$ with local and nonlocal components. Specifically, the transfer rate, as given from a standard statistical closure, is
$T_{k}=\sum_{\mathbf{k}^{\prime}}\left|\tilde{n}_{\mathbf{k}}\right|^{2}\left|\tilde{n}_{\mathbf{k}^{\prime}}\right|^{2}\left[A_{N L}\left(k, k^{\prime}\right)+A\left(k, k^{\prime}\right)\right]+\sum_{\mathbf{p}+\mathbf{q}=\mathbf{k}}\left|\tilde{n}_{p}\right|^{2}\left|\tilde{n}_{q}\right|^{2} A(p, q)$

where

$A_{N L}\left(k, k^{\prime}\right)=$ const. $\left(k \times k^{\prime} \cdot z\right)^{2} L_{\mathbf{k}, \mathbf{k}^{\prime}}^{-1}\left(\mathbf{k}^{\prime 2}{ }^{2}-\mathbf{k}^{2}{ }^{2}\right)$,

$A\left(k, k^{\prime}\right)=$ const. $\left(\mathbf{k} \times \mathbf{k}^{\prime} \cdot \mathbf{z}\right)^{2} L_{\mathbf{k}, \mathbf{k}^{\prime}}^{-1} \mathbf{k}_{\mathbf{y}}{ }^{2}$,

are coupling coefficients, and reflection symmetry in $\mathrm{k}_{\mathbf{y}}$ has been assumed for convenience in this discussion. Aside from the $\mathrm{E} \times \mathrm{B}$ geometrical factor $\left(\mathbf{k} \times \mathbf{k}^{\prime} \cdot \mathbf{z}\right)^{2}$ and the nonlinear response time $L_{k}^{-1}, k^{\prime}$ (to be defined in the next section), these coefficients are governed by the factors $\left(k_{y}{ }^{2}-k_{y}{ }^{2}\right)$ and $k_{y}{ }^{2}$. The factor in $A_{N L}$ indicates transfer which is negligible for $\mathrm{k}$ comparable to $\mathrm{k}$ ', but large for disparate values, i.e., transfer which is nonlocal. Also, the sign of $A_{N L}$ is such that energy is depleted from the mode $k$ when $k_{y}{ }^{2}>k_{y}{ }^{2}$, and deposited into that mode when $\mathbf{k}_{\mathbf{y}}{ }^{2}<\mathbf{k}_{\mathbf{y}}{ }^{2}$. This clearly implies transfer to large wavenumber. By contrast, the factor in $A$ is positive definite, indicating transfer which is always out of (rather than into) the mode $k$, and independent of the relative positions of $k$ and $\mathbf{k}^{\prime}$ in wavenumber space. (Note that for this term, nonlocal transfer is not precluded.)

The strong interaction and direct energy exchange between modes of widely disparate wavenumbers suggested by $A_{N L}$ is a fundamental departure from the cascade dynamics implicit in steady state spectra formulated according to the similarity concepts of Kolmogorov. If nonlocal transfer dominates, it is possible that the steady state wavenumber spectrum in an inertial range will strongly deviate from a self-similar spectrum. It is difficult to determine precisely the relative magnitudes of local and nonlocal transfer from renormalized equations without knowing the spectrum. Moreover, nonlocal transfer may be offset by the term A, which is not restricted to purely local triads. In the simulations, nonlocil and local transfer rates are directly measured throughout the relaxation. 
The dominant nonlinearity governing mode coupling at long wavelengths is the ExB nonlinearity. This nonlinearity is anisotropic with respect to the two cross-field directions. The anisotropy carries over to the closure equations, where highly anisotropic transfer, particularly nonlocal, is evident. In contrast, the equilibrium spectrum, derived from a single isotropic invariant (energy) is isotropic in the two cross-field directions. This seeming contradiction is examined in detail from the simulation results in order to determine the degree of anisotropy in the spectrum, and local and nonlocal transfer rates.

The results of this chapter are now summarized. Inertial energy transfer by trapped ion convective cell turbulence has been examined numerically for a 2-D Kadomtsev-Pogutse fluid model. Numerical solution of the equaticns was accomplished with a spectral code containing up to $41 \times 41$ modes. The spectrum evolution and transfer rate time histories of spectra initially peaked at low wavenumber were observed with two distinct regimes of evolution in evidence. In the first, there is a strong transfer of energy to high wavenumber occurring over several eddy turnover times and resulting in the relaxation of the spectrum to a configuration with noticeable peaking at high $\mathrm{k}_{\mathrm{y}}$ ( $\mathrm{y}$ is the cross-field direction perpendicular to the inhomogeneity in density). Nonlocal transfer in $\mathrm{k}_{\mathrm{y}}$ plays an important role in the relaxation process and is responsible for the peaking in $\mathbf{k}_{\mathbf{y}}$. Enstrophy increases throughout this regime. In the second regime the spectrum further relaxes to an approximately equipartitioned state under the action of sloshing in wavenumber space. The final spectrum is roughly consistent with the predictions of equilibrium statistical mechanics based on a single invariant corresponding to the internal energy. A slight peaking in $\mathrm{k}_{\mathrm{y}}$ is apparent in this time-asymptotic spectrum. The peaking represents a minor deviation from the predicted equilibrium spectrum, but one which strengthens, rather than weakens, the equilibrium statistical mechanics prediction that energy transfer is to small scale. For an initial spectrum which is flat, net transfer to small scale in the $\mathrm{k}_{\mathrm{y}}$ direction is again evident, but is weaker than that of the peaked spectrum case. This transfer is highly nonlocal, producing a spectrum which is slightly peaked in $\mathbf{k}_{\mathrm{y}}$ and similar to the spectrum of the sloshing regime when reached from a peaked initial condition. The flat initial spectrum case is important because stationary turbulence driven at long wavelengths, damped at short wavelengths, and having an inertial range in intermediate scales, results in a stationary spectrum which is only weakly peaked at low $k$.

Nonlocal transfer in $\mathrm{k}_{\mathbf{y}}$ derives from the direct coupling of modes which are widely separated in wavenumber space. For initial spectra with indices $\alpha$ less that 3 , where $\left.\ln \right|^{2}=$ $\mathrm{k}_{\mathrm{y}}{ }^{-\alpha}$, the nonlocal transfer in $\mathrm{k}_{\mathrm{y}}$ dominates local transfer, thus invalidating Kolmogorov similarity range arguments for inertial range transfer. For $\alpha>3$, the coupling between disparate scales is strongly reduced by the large amplitude disparity and transfer is dominantly local until the spectrum has relaxed to $\alpha \sim 3$. The local and nonlocal rates are comparable in the $\mathbf{k}_{\mathbf{x}}$ direction. This represents a pronounced anisotropy in the spectral transfer of energy. Because the local transfer tends to be isotropic, anisotropies in the spectrum are less pronounced.

The remainder of this chapter is organized as follows. The basic model, its properties and the basic computational procedure are presented in Sec. II. In Sec. III, the equiliorium spectrum and similarity-range stationary spectrum are derived and the prediction for spectral transfer direction is formulated. The simulation results are detailed in Sec. IV, and conclusions are given in Sec. $\mathrm{V}$.

\subsection{Basic Equations and Computational Procedure}

In order to study nonlinear processes in detail, a simple model is used. This model, based on Kadomtsev-Pogutse equations, treats dissipative trapped ion convective cell turbulence as a turbulent fluid described by a single scalar related to both the fluctuating 
density and the flow stream function (electrostatic potential). This model therefore allows contact with the considerable body of knowledge existing for Navier-Stokes turbulence and similar fluid plasma models such as the Hasegawa-Mima equation ${ }^{11}$ and its dissipative analogs 12 . At the same time, the model is sufficiently complete to capture many essential elements of trapped ion turbulence. The model equation is given by

$\frac{\partial \tilde{n}}{\partial \mathrm{t}}+D \frac{\partial^{2} \tilde{\mathbf{n}}}{\partial \mathrm{y}^{2}}+\frac{V_{\mathrm{D}}}{2} \frac{\partial \tilde{n}}{\partial \mathrm{y}}+v_{\text {eff, }, \overline{\mathrm{n}}}-\frac{4 \mathrm{~L}_{\mathrm{n}} \mathrm{D}}{\varepsilon^{1 / 2}} \nabla \frac{\partial \tilde{\mathrm{n}}}{\partial \mathrm{y}} \times \mathbf{z} \cdot \nabla \tilde{\mathbf{n}}=0$,

where $\overline{\mathbf{n}}=\varepsilon^{1 / 2} \mathrm{n}^{t / n_{0}}$ is the normalized trapped ion density, $V_{D}=\varepsilon^{1 / 2}\left(c T_{i} / e B\right) L_{n}^{-1}$ is the effective diamagnetic drift velocity for trapped ions, $D=V_{D}^{2 / 4 v_{e f f, e}}$ is an inverse diffusivity describing the destabilization of trapped ion modes by electron collisions, $v_{\text {eff, } i}$ is the effective collision frequency of ion-ion collisions, $v_{\text {eff }} j_{j}=v_{j} / \varepsilon, \varepsilon=r / R$ is the inverse aspect ratio parameterizing the fraction of trapped particles, and $L_{n}$ is the density gradient scale length. This equation incorporates the dynamics of both trapped electrons and trapped ions with quasineutrality providing the link between their densities. The turbulent fluid flow is the ExB flow. This flow couples to the density fluctuations through the ExB advection of the mean density gradient.

The electron dynamics incorporates adiabatic (passing) and nonadiabatic (trapped) electrons. As a result of the very low frequency of the fluctuations, the trapped electrons are collisional, i.e., trapped electrons experience multiple collisions over a fluctuation period. Consequently, electron dynamics are governed by collisional scattering as opposed to nonlinear advection. This results in a linear relation between the fluctuation source (given by the $\mathrm{E} \times \mathrm{B}$ advection of the average density) and the trapped electron density. Trapped electrons are therefore laminar, with a density which is proportional to the potential and $90^{\circ}$ out of phase due to the collisions. Eiectron collisions access the density gradient free energy through an inverse damping process and thus provide the basic instability which feeds the turbulence.

The combination of electron and ion densities into a single field using the laminar electron response constitutes an "i $i$ " approximation $\left(\tilde{n}_{e} \sim i \delta \phi\right.$, where $\delta=4 L_{n} D / \varepsilon^{1 / 2}$ is the nonadiabatic electron response). It is worth noting that because electron inertia is negligible, the nonadiabatic electron response, or function $\delta$, has no explicit dependence on the irequency. Thus, the need to approximate the nonadiabatic response by evaluating an explicit frequency dependence at $\omega_{*}$ or a linear frequency does not arise. Explicit frequency dependence in other types of drift-wave fluctuations (collisionless trapped electron modes, universal modes, etc.) represents a serious shortcoming of the i $\delta$ approximation, but one which does not occur in the present case.

The ion response is hydrodynamic and consists of $\mathrm{E} \times \mathrm{B}$ advection, the polarization drift, and ion-ion collisions. The latter affects the fluctuations at very long wavelengths providing a low $\mathrm{k}$ cutoff for the instatiing. The long wavelengths of the fluctuations restrict the extent to which the polarizatiou drift plays any role in the dynamics. As a consequence, the fluctuations are essentially nondispersive in the energy containing scales fed by the instability. Similarly, the polarization drift nonlinearity $\left(n_{0} \nabla \cdot v_{p}(1)\right.$ where $v_{p}(1)=B_{0}{ }^{-1}$ $(c / e) Z \times v_{E} \cdot \nabla v_{E}$ and $\left.v_{E}=-\nabla \phi \times z\right)$, familiar from the Hasegawa-Mima equation, is small compared to the $\mathrm{E} \times \mathrm{B}$ nonlinearity $\left(\mathrm{v}_{\mathrm{E}} \cdot \nabla \tilde{\mathbf{n}}_{\mathbf{i}}\right)$. The $\mathrm{E} \times \mathrm{B}$ nonlinearity is nonzero only through the nonadiabatic electron response and produces dissipative coupling which breaks enstrophy conservation ${ }^{12}$. The EXB nonlinearity dominates at long wavelengths where kp $<\delta$ ( $\rho$ is the ion gyro-radius at the electron temperature). The present study is concerned with the long wavelength limit, so only the EXB nonlinearity is retained in the model Because energy transfer is toward small scales in regions dominated by the ExB nonlinearity (a principal conclusion of this chapter), energy eventually reaches regions where the polarization drift nonlinearity becomes important and uther effects occur, such as 
coupling to smaller wavelength trapped electron modes. Future work will address the dynamics at shorter wavelengths where the two nonlinearities participate in the transfer process.

As indicated previously, the Kadomtsev-Pogutse equation, Eq. (2.3), accurately represents many essential elements of trapped ion turbulence. These include the correct electron dissipation-induced linear growth rate of the driving instability. The linear frequency is also correctly represented as nondispersive, in the direction of the electron diamagnetic drift, and offset from the diamagnetic frequency by the trapped particle fraction. Energy transfer is appropriately governed by the EXB nonlinearity. This model does not account for parallel dynamics (and hence radial mode structure) or coupling to short wavelength fluctuations.

The computational method employed is spectral. Thus the time evolution of Fourier modes (coupled by the convolution sum of the EXB nonlinearity) is solved according to

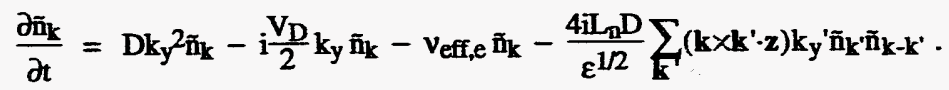

A truncation of Fourier space limits the spatial resolution of the solution. Time advancing is accomplished using a Gear-type solver with an explicit Jacobian. The code is purely spectral, treating Eq. (2.4) as it is written, as opposed to pseudo-spectral, which uses fast Fourier transforms to calculate the convolution term in real space where the fields are a simple product. The use of a spectral method limits the resolution relative to that possible with a pseudo-spectral code; however, subtle issues involving dealiasing are thus avoided. In particular, pseudo-spectral methods require dealiasing, a process which distorts the Fourier modes in the shorter wavelength part of the spectrum. The portion of the spectrum which is usable is affected by anisotropies in the nonlinear coupling, and has subtleties which depend on whether the spectrum represents second order moments such as energy, or higher order moments ${ }^{13}$. In the present work, the energy and enstrophy transfer rates are computed. These quantities are calculated from triplet comrelations. The effect of dealiasing on third order correlations with anisotropic coupling is not well documented and for the purpose of developing such diagnostics, the spectral environment provides easier implementation and more reliable interpretation. The results presented here are obtained using a maximum of $41 \times 41$ modes. For the study of spectrum relaxation and energy transfer in turbulence which is undriven/undamped, this resolution is adequate, as verified by changing the number of modes.

A key feature of the computations reported in this chapter is the calculation of the energy and enstrophy transfer rates $T_{k}$ and $U_{k}$ in wavenumber space. Energy and enstrophy are defined as $\Sigma_{k}\left|\tilde{n}_{k}\right|^{2}$ and $\Sigma_{k} k^{2}\left|\tilde{n}_{k}\right|^{2}$. Thus, rates of energy and enstrophy transfer from a given mode $k$ are obtained by multiplying the last term of Eq. (2.4) by $\tilde{n}_{\mathbf{k}}$ * and $\mathbf{k}^{2} \tilde{\mathbf{n}}_{\mathbf{k}} *$ giving respectively

$T_{\mathbf{k}}=\frac{4 i L_{\mathbf{n}} \mathrm{D}}{\varepsilon^{1 / 2}} \operatorname{Im} \sum_{\mathbf{k}}\left(\mathbf{k} \times \mathbf{k}^{\prime} \cdot \mathbf{z}\right) \mathbf{k}_{\mathbf{y}}{ }^{\prime} \tilde{\mathbf{n}}_{\mathbf{k}^{\prime}} \tilde{\mathbf{n}}_{\mathbf{k}-\mathbf{k}^{\prime} \tilde{\mathbf{n}}_{\mathbf{k}}{ }^{*}}$

and



These quantities are evaluated at each step of the computation yielding instantaneous rates of transfer between the mode $\mathrm{k}$ and all other coupled modes in the spectrum. $T_{k}$ positive represents a net flow of energy into the mode $k$; $T_{k}$ negative represents net outflow. Because energy is conserved by the nonlinear transfer, the energy flow into or out of a mode must be equal to the flow out of or into all remaining modes. Enstrophy, on the other hand, is not conserved. If energy is transferred conservatively to short wavelength, as 
occurs in 3-D Navier-Stokes turbulence, it follows from simple dimensional arguments that enstrophy will be generated. If energy flows to long wavelength, the converse will hold. Consequently, the evolution of total enstrophy, obtained from the time history of $\Sigma_{k} U_{k}$, provides an additional indicator of energy transfer direction and is the principal enstrophy diagnostic utilized.

The closure representation of the energy transfer rate [Eq. (2.2)] follows from Eq (2.5) by a straight forward iteration on each of the density fluctuation factors. Consistent with quasi-Gaussian statistics, the iterated density factors, or driven fluctuations, are directly excited by a single triplet interaction, with the remaining triplets acting to nonlinearly decorrelate the interaction. The directly acting triplet is the one yielding closure, e.g.,

$\mathbf{L}_{\mathbf{k}-\mathbf{k}^{\prime}} \tilde{\mathbf{n}}_{\mathbf{k}-\mathbf{k}^{\prime}}=-\frac{4 \mathrm{i} \mathbf{L}_{\mathbf{x}} \mathbf{D}}{\varepsilon^{1 / 2}}\left(\mathbf{k}^{\prime} \times \mathbf{k} \cdot \mathbf{z}\right)\left(\mathbf{k}_{\mathbf{y}}+\mathbf{k}_{\mathbf{y}}\right) \tilde{\mathbf{n}}_{\mathbf{k}} \tilde{\mathbf{n}}_{\mathbf{k}^{\prime}}$

where $L_{k-1} \mathbf{k}^{\prime}$ is the nonlinearly broadened propagator, consisting of linear growth, damping, diamagnetic rotation, and the nonlinear decorrelation. Carrying out this procedure yields

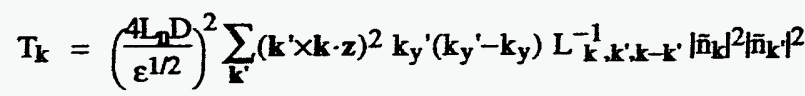

$+\left.\left(\frac{4 L_{n} D}{\varepsilon^{1 / 2}}\right)^{2} \sum_{\mathbf{k}^{\prime}}\left(\mathbf{k}^{\prime} \times \mathbf{k} \cdot \mathbf{z}\right)^{2}\left(\mathbf{k}_{\mathbf{y}^{\prime}}{ }^{2}-\mathbf{k}_{\mathbf{y}^{2}}{ }^{2}\right) \mathrm{L}_{\mathbf{k}, \mathbf{k}^{\prime} \cdot \mathbf{k}-\mathbf{k}^{\prime}}^{-1}\left|\tilde{n}_{\mathbf{k}} \mathcal{R}^{2}\right| \tilde{n}_{\mathbf{k}^{\prime}}\right|^{2}$

$+\left(\frac{4 L_{n} D}{\varepsilon^{1 / 2}}\right)^{2} \sum_{p+q=k}(\mathbf{p} \times q \cdot z)^{2} p_{y}\left(p_{y}-q y\right) L_{\mathbf{k}, p \cdot q}^{-1}\left|\tilde{n}_{p}\right|^{2}\left|\tilde{n}_{q}\right|^{2}$

where $L_{k, k^{\prime}, k-k^{\prime}}^{-1}=i\left(\omega_{k^{\prime}}+\omega_{k-k^{\prime}}+\omega_{-k}-\gamma_{k^{\prime}}-\gamma_{k-k^{\prime}}-\gamma_{-k}+\Delta \omega_{k^{\prime}}+\Delta \omega_{k-k^{\prime}}+\Delta \omega_{-k}\right)$ is the triplet interaction correlation time, $\gamma_{\mathbf{k}}=\mathrm{Dk}_{\mathbf{y}}{ }^{2}-v_{\mathrm{eff}, \mathrm{e}}$ is the linear growth rate, and $\Delta \omega_{\mathrm{k}}=$ $\left[\left(4 L_{n} D\right)^{2 / \varepsilon}\right] \sum_{k^{\prime}}\left(k^{\prime} \times k \cdot z\right)^{2} k_{y^{2}}{ }^{2} L_{k, k^{\prime} \cdot k-k^{\prime}} \mid \bar{n}_{k} t^{2}$ is the nonlinear decorrelation rate.
The first term of Eq- (2.7) comes from the iteration of $\tilde{n}_{k-k}$ and describes advection of density by the turbulent flow. The third term arises from the iteration of $\tilde{\mathbf{n}}_{\mathbf{k}}{ }^{*}$ and incorporates the incoherent transfer process neglected in one-point closures ${ }^{14}$. The second term is obtained by iterating on $\overline{\mathbf{n}}_{\mathbf{k}^{\prime}}$, the fluctuation representing the turbulent flow $\left[\widetilde{\mathbf{v}}_{\mathbf{k}^{\prime}} \propto\right.$ $\left.i k_{y} \tilde{n}_{\mathbf{k}^{\prime}}\right]$. Consequently, this term provides for the self-consistent back reaction of the density fluctuations on the turbulent flow. In closures of Vlasov-Poisson systems, this term represents the renormalization of the shielding cloud and is frequently neglected becanse it requires the solution of a nonlinear Poisson equation. In fluid treatments it has a similar meaning through the correspondence between flow and potential which underlies EXB motion. Likewise, it is often neglected in order to avoid a nonlinear eigenvalue problem. The nonlocal transfer produced by this term can be thought of as the interaction between large scale flow (shielding potential) and smaller scale density fluctuations. As noted in the introduction, the nonlocal transfer is unequivocally toward small scale.

In order to reduce the data required to establish the direction of energy transfer and examine other properties of the transfer process, the transfer into or from bands in wavenumber space is measured by summing Eqs. (2.5) and (2.6) over selected $k$ values. The bands are chosen to represent slices in $k_{x}$ and $k_{y}$ in order to be sensitive to any anisotropies in the wavenumber space transfer. With the band structure, it is possible to track local energy flow in $k$-space through adjacent bands. Nonlocal transfer is also easily observed. The definition of local versus nonlocal transfer is somewhat difficult to quantify. Typically, nonlocal transfer is used to signify exchange of energy with modes in the discrete spectrum which are displaced by more than two or three wavenumbers, ie., an interaction with modes which are not nearest neighbors. However, with such a definition, observed nonlocal transfer could still be compatible with self-similar transfer, provided it admitted a similarity range with a number of these nonlocal steps. In order to identify energy flow which is incompatible with a similarity range, nonlocal transfer will be defined as energy 
exchange in interactions spanning more than half (or occasionally one third) of the inertial range. Local transfer will be the difference of the total transfer and the nonlocal transfer, i.e., the flow to modes which are not removed by approximately half of the $k$-space extent.

\subsection{Equilibrium and Similarity Range Spectra}

In this section, the prediction that trapped ion turbulence produces a transfer of energy to short wavelength ${ }^{4}$ is reviewed. This prediction is based on a comparison of the equilibrium spectrum calculated from statistical mechanics, and the stationary spectrum describing the distribution of energy in a driven saturated state. The equilibrium spectrum is derived using the statistical probability disuribution function for a canonical ensemble consistent with the known invariants of the nonlinearity. In applying these statistical tools, it is assumed that turbulent transfer in the steady state is in the circction in wavenumber space which would tend to drive the spectrum to the equilibrium configuration if the driving and damping were tumed off. The testing of this assumption is one of the objectives of the present study. Clearly, application of this method requires knowledge of the stationary spectrum established by the turbulently moderated balance of driving and damping. In this chapter, the simplest stationary spectrum, consistent with a Kolmogorov-type similarity range, will be adopted as a benchmark. Because the validity of a similarity range spectrum is cast into doubt by the results of the next section, the issue of the appropriate stationary spectrum and its role in the prediction of transfer direction will be revisited at that time.

In the absence of driving and damping, a single nontrivial quadratic invariant is admitted by the Kadomtsev-Pogutse equation. This invariant is the internal euergy, $\int d^{2} x \tilde{n}^{2}$, whose conservation follows from the fact that

$\int \nabla \frac{\partial \tilde{\mathbf{n}}}{\partial \mathbf{y}} \times \mathbf{z} \cdot \nabla \tilde{\mathbf{n}} \mathbf{d}^{2} \mathbf{x}=0$

A second integral, $\int d^{2} x \partial \tilde{n}^{2} / \partial y$, is also conserved by the nonlinearity. However, by symmetry, this integral is identically zero for all time, and therefore imposes no constraint on the cascade dynamics. Enstrophy, an invariant of the 2-D Navier-Stokes and HasegawaMima equations, is not conserved by the EXB nonlinearity. The nonconservation of enstrophy arises from the nonadiabatic electrons 12 .

With energy as the only nonzero quadratic invariant, the canonical probability distribution of equilibrium states is

$P=\exp \left[-\frac{\beta}{2} \sum_{\mathbf{k}}\left|\tilde{\mathbf{n}}_{\mathbf{k}}\right|^{2}\right]$

where $B$ is an effective inverse temperature. The spectrum is given by the expectation value of the energy in a mode $k_{j}$ yielding

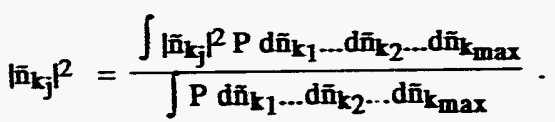

Equation (2.10) predicts an eguipartition of the energy,

$\left|\tilde{n}_{\mathbf{L}}\right|^{2}=\frac{1}{\beta}$,

corresponding to a flat spectrum in the 2-D wavenumber space. In addition to equipartition, the equilibrium spectrum is isotropic, a feature which follows from the fact that the lone nonzero quadratic invariant has no explicit anisotropy.

As stated previonsly, a similarity range stationary spectrum is calculated for comparison with the equilibrium spectrum. Assuming that unstable modes are confined to a 
localized region of wavenumber space where $\omega<\omega_{b i}$, the balance of nonlinear transfer of energy $\widetilde{n}^{2}$ at each scale in the spectrum with the net energy input rate requires that $\varepsilon=$ $\mathbf{k}^{3} \tilde{\mathbf{n}}_{\mathbf{k}}{ }^{3}$, where $\varepsilon$ is the fixed energy input rate and isotropy has been assumed, i.e., $\partial / \partial \mathbf{x}=$ $\partial / \partial \mathrm{y} \rightarrow \mathrm{k}$ Solving for $\tilde{\mathrm{n}}_{\mathbf{k}}{ }^{2}$ and expressing in terms of an isotropic integral distribution for a single wavenumber $k\left[\int E(k) d k=\tilde{n}^{2}\right]$, the spectrum is

$E(k)=\varepsilon^{2 / 3} \mathbf{k}^{-3}$

If the spectrum is anisotropic ( $\partial / \partial x \neq \partial / \partial y$ ), the balance of energy input rate with spectral transfer requires that $\varepsilon=k_{y}{ }^{2} k_{x} \tilde{n}_{k}{ }^{3}$. Writing the spectrum as an imtegral distribution in two wavenumbers $\left[\int E\left(k_{x}, k_{y}\right) d k_{x} d k_{y}=\tilde{\mathbf{n}}^{2}\right]$ yields

$E\left(k_{x}, k_{y}\right)=\varepsilon^{2 / 3} k_{x}-5 / 3 k_{y}-7 / 3$

Note that the second spectrum is consistent with the first and reduces to it when $k_{x}=k_{y}$. It has an additional power of $5^{-1}$ because it is a distribution of energy in a * o-dimensional wavenumber space. In contrast to the equilibrium spectrum, both similarity range spectra are peaked at long wavelength. The anisotropy in $\mathbf{k}_{\mathrm{x}}$ and $\mathrm{k}_{\mathrm{y}}$ of Eq. (2.13) arises directly from the anisotropy of the EXB nonlinearity. These spectra follow for an inertial cascade, regardless of its direction in wavenumber space. Assuming that the nonlinear interations of the inertial range attempt to drive the system toward equilibrium, transfer will be in such a direction as to establish the equilibrium spectrum, $E \sim \beta^{-1}$. On this basis, transfer is predicted to be toward short wavelength. If a similarity range does not exist, but the stationary spectrum of driven turbulence remains peaked at the long wavelength, the transfer is again predicted to be toward shor wavelength.

The transfer of energy to short wavelength is a consequence of the existence of a single quadratic invariant. By contrast, the 2-D Navier-Stokes and Hasegawa-Mima equations have two invariants and thus a canonical probability distribution function which depends on the two integrals. The equilibrium spectra calculated from this distribution function have enstrophy weighted toward short wavelengths and energy weighted toward long wavelengths. Consequently, enstrophy in these systems is predicted to flow to short wavelengths and energy to long wavelengths.

\subsection{Computational Results}

The results reported in this section come principally from numerical experiments in which turbulence is initialized with finite amplitude in a variety of spectral distributions and allowed to evolve with no forcing or dissipation. Initial spectra range from $\mathrm{k}^{-1}$ to $\mathrm{k}^{-6}$, bracketing the similarity range stationary values of $k_{x}-5 / 3 k_{y}-7 / 3$. Evolution is tracked for as many as 100 eddy turnover times. In all cases, the initial spectra relax to an identical time-asymptotic configuration. The instantaneous flow during the spectrum evolution is measured to indicate the flow which would occur in a driven/damped staticnary situation having a spectrum like the instantaneous spectrum during relaxation. The time history of energy is calculated throughout the runs as a check on the numerics. Energy is found to be conserved to better than one part in $10^{8}$ for tens of eddy turnover times. The nonlinear transfer diagnostics have also been benchmarked against hand calculations for a small $k$ space in order to verify their accuracy.

The time-asymptotic spectrum is found to be flat, in very good agreement with the predicted equilibrium spectrum. This agreement is remarkable given that the nonlinearity which drives the relaxation and the ineasured wavenumber space flow are anisotropic; yet the final spectrum is isotropic and in good agreement with a prediction which takes no account of the anisotropy in the nonlinearity. Figures 2.1-2.3 show a sequence of spectra, starting with an initial spectrum given by $E(k)=k^{-4}$, an intermediate configuration which 

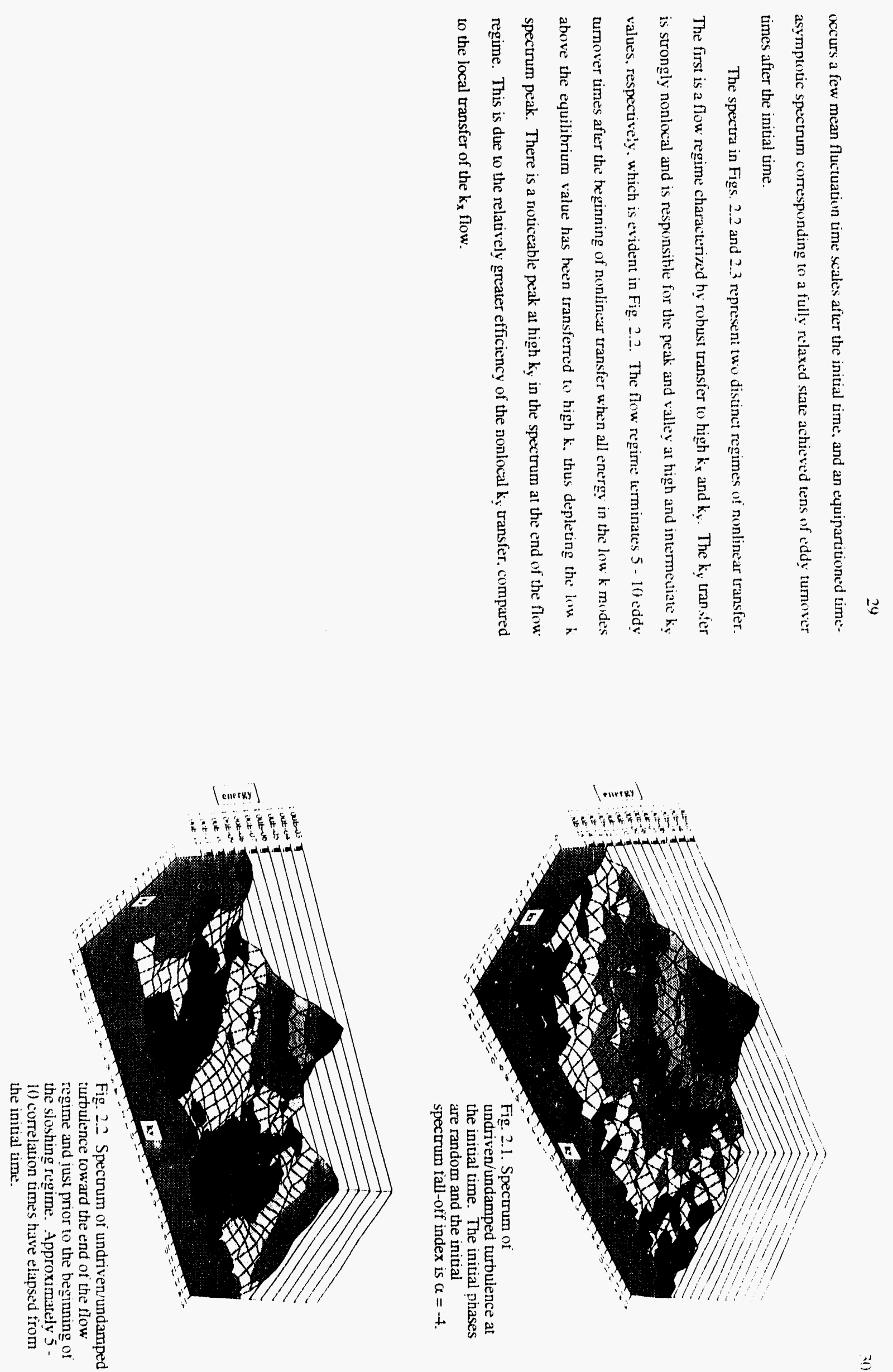


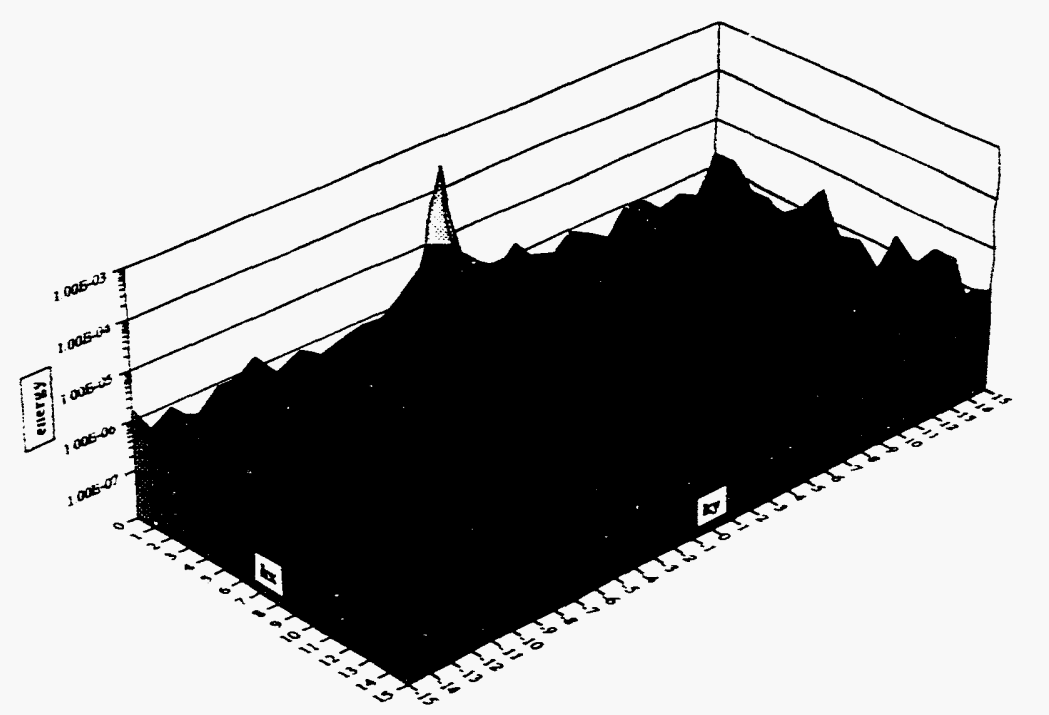

Fig. 23 Spectrum of undrivenhundamped turbulence in the sloshing regime. The spectrum has been averaged over several correlation times. This spectram is in the time-asymptotic (relaxed) configuration. (The central peak is the mode $\mathbf{k x}=\mathbf{k y}$ $=0$. Because this mode does not couple to any other mode, it retains its initial energy.)
Because the spectrum at the end of the flow regime is anisotropic and not quite at the equilibrium configuration, a second regime ensues, characterized by sloshing of e-argy in wavenumber space. The sloshing regime exhibits rapid transfer of energy betweeu modes but with little net transfer. The spectrum is quickly isotropized and flattened by the sloshing. The time-asymptotic spectrum is nearty flat with a slight peak at high $\mathbf{k}_{\mathbf{y}}$. The sloshing interaction incorporates a wide range of time scales, from an eddy turnover time to hundreds of eddy turnover times. The sloshing motion roughly produces the equipartitioned spectrum predicted by equilibrium statistical mechanics. However, this regime is probably not relevant to the transfer occurring in a driven stationary state. Insofar as the nonlinear transfer of the steady state is concemed, it is essentially an artifact of the finite (truncated) $k$-space coupled with the efficient nonlocal transfer, and the fact that there is no dissipation at short wavelengths to absorb the energy nonlinearly transferred from long wavelengths.

While the two regimes of nonlinear transfer have a noticeable effect on the spectrum. their properties are most clearly seen in the energy transfer and enstrophy production diagnostics. Figure 2.4 shows the net energy transfer from bands of constant $k_{y}$ and $k_{x}$ in both the long and short wavelength parts of the spectrum. The flow regime is clearly apparent as the period over which there is a continuous oufflow from low $k$, as evidenced by the negative value of $T_{k}$ for the long wavelength bands, and an inflow to high $k$, as seen in the positive value of $T_{k}$ for the short wavelength bands. After $t=2.5$, the sloshing regime is reached and the transfer is oscillatory with no net sign apparent. Figure 2.5 indicates that the enstrophy production rate is positive throughout the flow regime and saturates at the transition to the sloshing regime. Positive enstrophy production results from the conscrvative transfer of energy from long to short wavelength, with the net increase of enstrophy proportional to the ratio $k_{\max }{ }^{2} / k_{\min }{ }^{2}$. The observed positive enstrophy production rate thus corroborates the results of the energy flow diagnostic. Note that the 

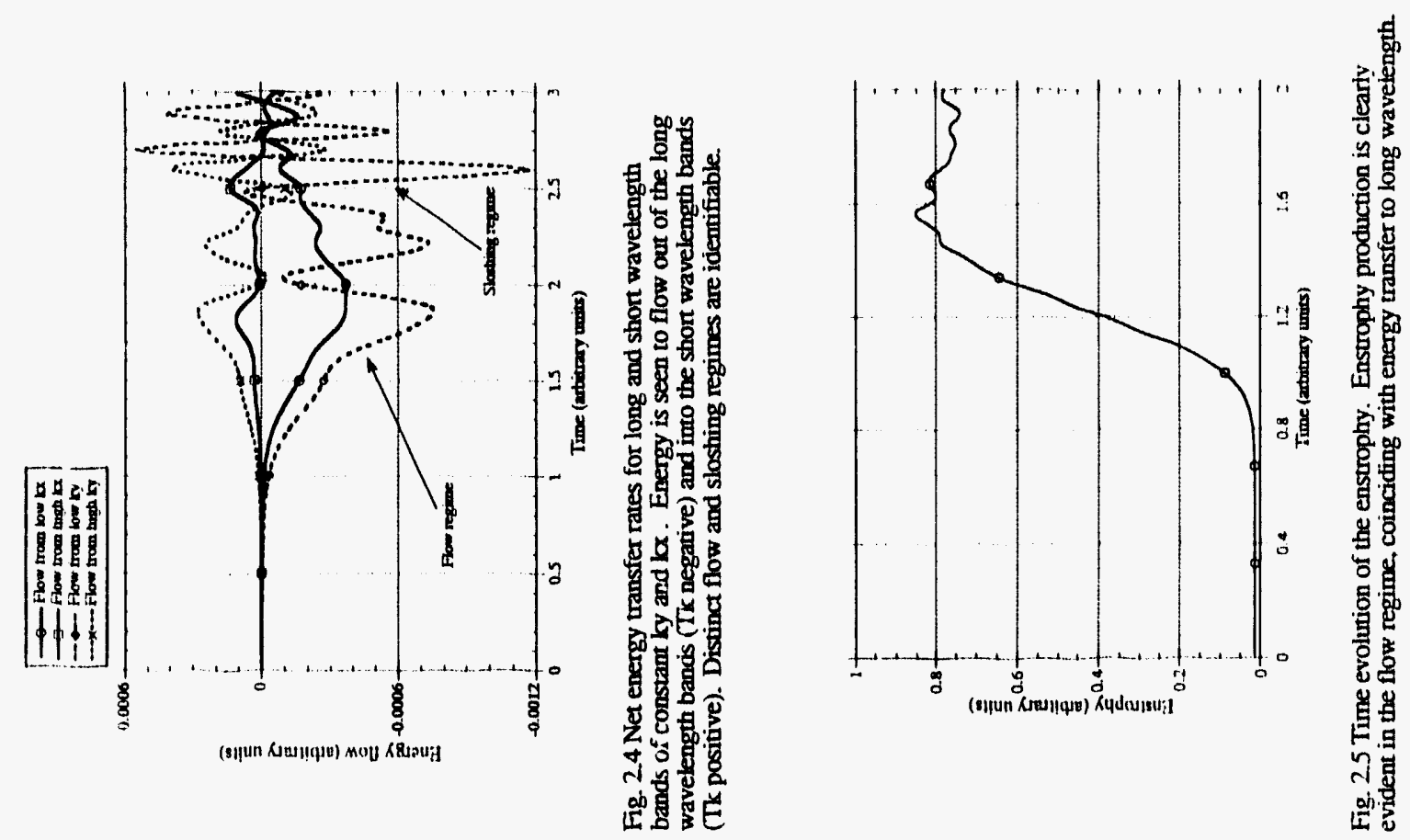

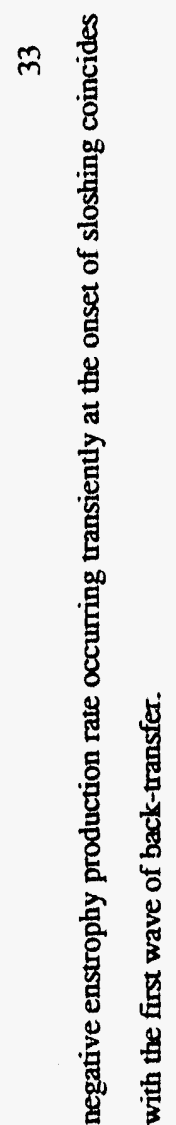


Nonlocal transfer and its magnitude relative to local transfer is now examined. Figure 2.6 shows the time evolution of local and nonlocal transfer rates from a long wavelength $k_{y}=$ constant band. By symmetry, transfer measured from a $k_{y}$ band is dominantly the transfer in the $k_{y}$ direction. From Fig. 2.6, the transfer in the flow regime is almost entirely nonlocal with a small local componemt. The initial spectrum in this case is $\mid \bar{n}_{1} f^{2} \sim k^{-4}$. Given the definition of nonlocal used in this study, this figure incicates that energy is efficiently passing directly from the low $k_{y}$ modes to modes which are more than one half of the wavenumber space removed. Stated in terms of the wavenumber triangles for the nonlinear interaction of $\mathbf{k}, \mathbf{k}^{\prime}$, and $\mathbf{k}-\mathbf{k}^{\prime}$, triangles which are highly elongated are strongly favored over equilateral triangles in carrying the energy to high $\mathrm{k}_{\mathbf{y}}$. By contrast, Fig. 2.7 indicates that the transfer from a long wavelength band with $k_{x}=$ constant is divided roughly equally between local and nonlocal components. There is therefore a pronounced anisotropy in the energy transfer process during the flow regime.

The anisotropy in transfer is also evident in the evolution of isodensity contours throughout the relaxation. Figures $2.8-2.10$ show isodensity contours at the initial time, in the flow regime, and in the sloshing regime when the spectrum has reached equilibrium in an average sense. As expected, the initial isodensity contour plot has just one or two structures, consistent with a spectrum peak at low $k$. As the spectrum evolves through the flow regime (excitation in the high $\mathbf{k}_{\mathbf{y}}$ modes is growing rapidly) the anisotropy in the transfer manifests itself in an anisotropy in the isodensity contours. Small scale structures appear in the $k_{\mathbf{y}}$ direction while few appear in the $\mathbf{k}_{\mathbf{x}}$ direction. This can be easily misinterpreted as stretching in the $k_{x}$ direction, but it should be remembered that the system is evolving by energy transfer from large scale to small scale structures. Hence, it is not stretching, but rather a break-up of the structure in the $\mathbf{k}_{\mathbf{y}}$ direction which is occurring. In the sloshing regime, the contours relax to an approximately isotropic configuration, consistent with the isotropic equipartitioned equilibrium spectrum.

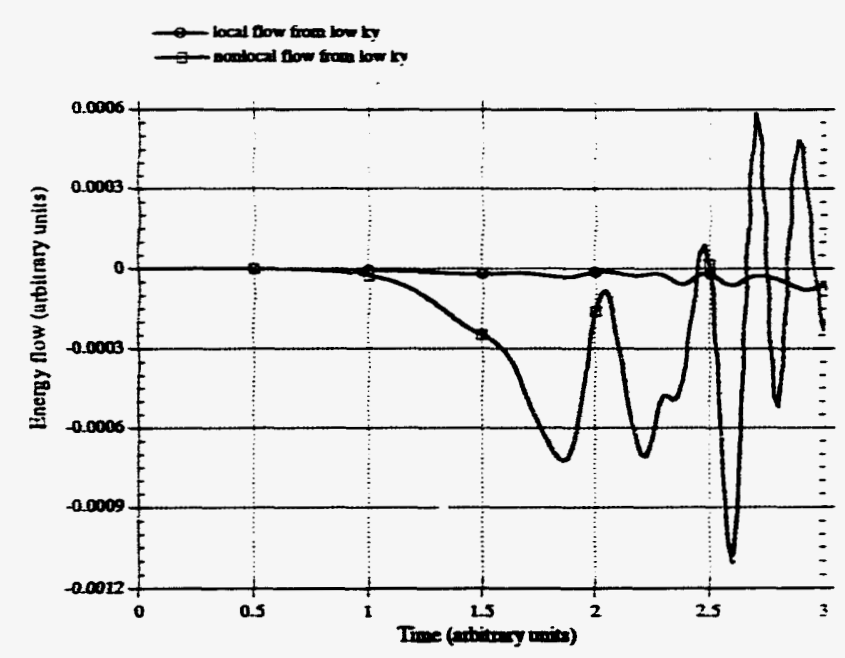

Fig. $2.6 \mathrm{Local}$ and nonlocal transfer rates in the ky direction. Transfer is clearly dominated by the nonlocal process, which here is defined as transfer beaween coupled modes separated by more than half of the wavenumber space.

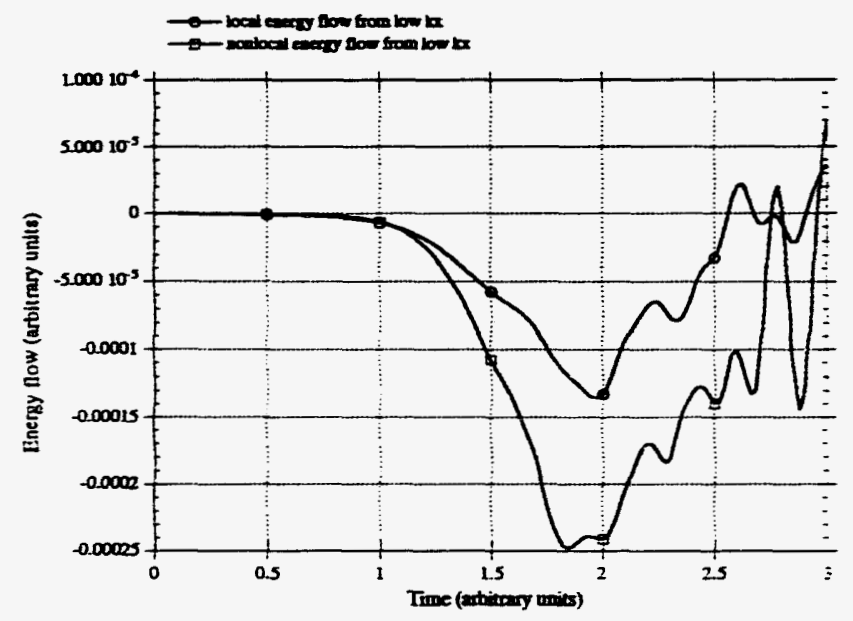

Fig. 27 Local and nonlocal transfer rates in the lox direction. The parity of local and nonlocal transfer rates in the lox direction and the disparity of local and nonlocal transfer rates in the ky direction indicates a clear anisotropy in the transfer process. 






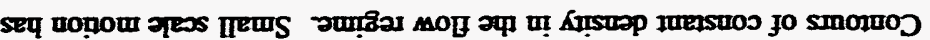

$62^{-3} \mathrm{x}$

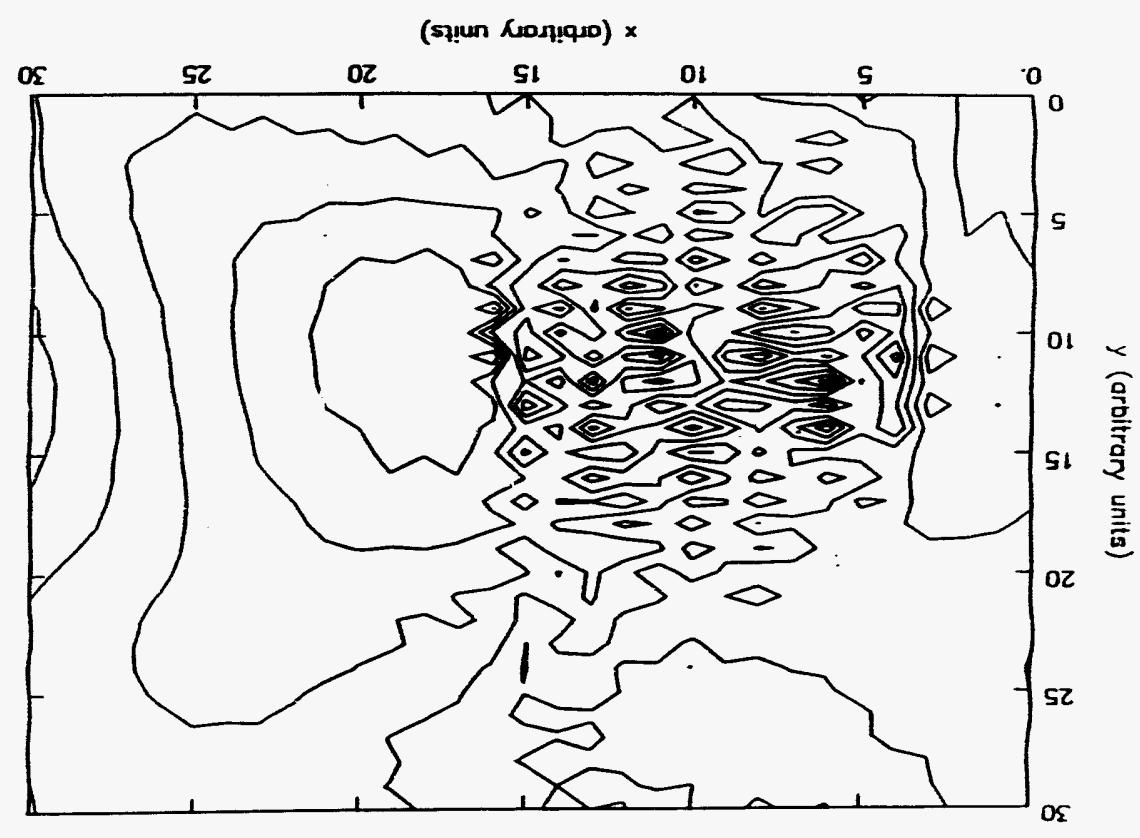

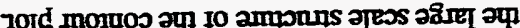

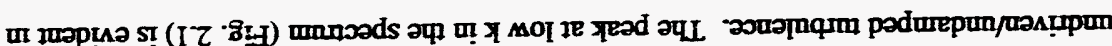

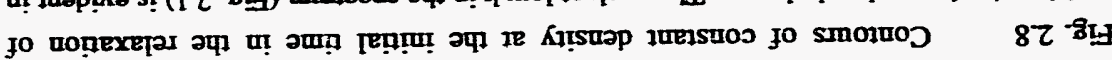

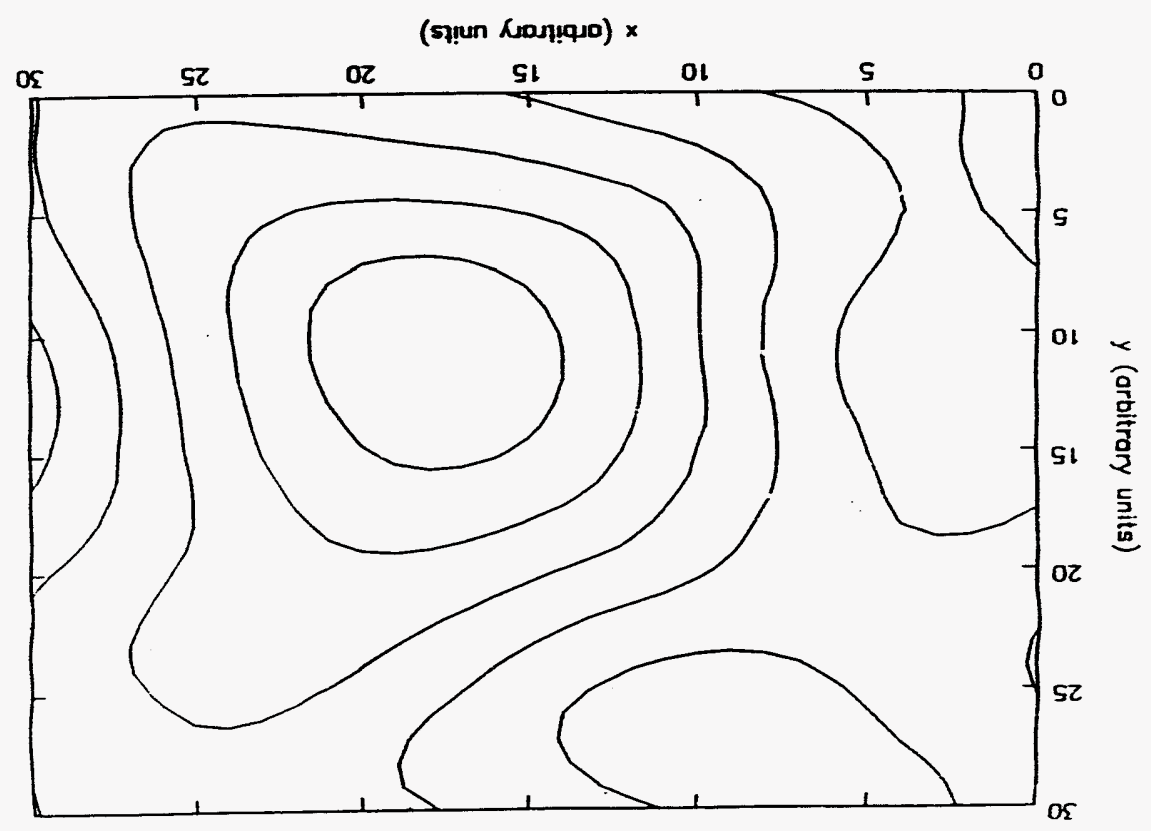






Fig. 2.10 Contours of constant density in the sloshing regime (see Fig. 2.3). Sloshing has isotropized the contours.
Transfer during the sloshing regime tends to be dominantly nonlocal in both the $\mathbf{k}_{\mathbf{x}}$ and $k_{\mathbf{y}}$ directions. This is understandable because the mechanism which keeps the transfer local in $k_{x}$ is suppressed in the sloshing regime. This mechanism is generally responsible for the locality of transfer in most systems, and stems from the amplitude dependence of the transfer coupled with the fact that the amplitude distribution generally falls off with higher $k$ If the spectrum decays as $n \sim n_{0} k-\alpha$, then the transfer coupling between modes $k$ and $k^{\prime}>$ $k$ goes as $T_{k} \sim n_{k}{ }^{2} n_{k}{ }^{2} \sim n_{0}{ }^{4} k^{-2 \alpha}\left(k^{\prime}-k\right)^{-2 \alpha}$ [using the closure representation, Eq. (2.2)] . Clearly, for $\alpha>0$, transfer to neighboring modes $k^{\prime} \geq k$ is strongly favored over transfer to distant modes $\left(k^{\prime}>k\right)$. However, when the spectrum becomes nearly flat and $\alpha=0$ (as in the case at the end of the flow regime), all modes have equal amplitude and the ratio of nonlocal to local transfer must increase.

This notion suggests that the strong nonlocal transfer in $\mathbf{k}_{\mathbf{y}}$ exhibited in Fig. 2.6 should be suppressed if the initial spectrum is made sufficiently steep. Indeed, when the initial spectrum slope exceeds $\mathbf{k}^{-3}$, nonlocal transfer is suppressed. In this case, transfer is local, producing a slow relaxation of the spectrum. Once the spectrum has relaxed to the $\mathrm{k}^{-3}$ slope, nonlocal transfer quickly begins and relaxatior; proceeds to the equilibrium spectrum at a much increased rate.

The dominance of nonlocal transfer over local transfer as evidenced in Fig. 2.6 is strongly incompatible with the notion of a self-sinitlar cascade which underlies the Kolmogorov-type spectrum [Eqs. (2.12) and (2.13)]. A similarity range cannot exist, at least in the $k_{y}$ direction, as energy : : transferred out of a mode and across the entire spectrum range in one correlation time. Indeed, the spectrum of steady state turbulence with driven modes at extremely low $k$, an intermediate inertial range over most of the spectrum, and a hyperviscosity at the highest wavenumbers, bears no resemblance to the similarity range stationary spectrum of Eq. (2.13). Figure 2.11 reveals the stationary spectrum to be nearly flat with slight peaking at low $k$ and strong quenching of the amplitudes in the 
dissipation range. While the inertial range is of limited extent in this spectrum (approximately 1 decade), the flatness is a robust feature independent of the strength of driving and the saturated turbulence level. Moreover, theoretical work based on the solution of a two-point equation likewise indicates that a flattening of the spectrum occurs as a result of the nonlocal transfer of the ExB nonlinearity 15 . Because the similarity range stationary spectrum differs so markedly from the numerical spectrum of stationary driven/damped turbulence, it is important to determine the direction of energy transfer in situations with spectra like that of Fig. 2.11. The transfer associated with the spectrum of Fig. 2.11 is found to be directed to large $\mathrm{k}$, as would be expected from the configuration of sources and sinks. Moreover, the transfer from an initially flat spectrum in the undriven undamped case is also toward high $\mathrm{k}$, producing a transfer rate history similar to that of Fig. 2.4, but with reduced magnitude. In this situation, nonlocal transfer proceeds in both directions whereas local transfer is directed to high $\mathrm{k}_{\mathrm{y}}$. Consequently, the spectrum develops a siight peak in $\mathbf{k}_{\mathbf{y}}$, and a sloshing regime ensues.

It is valid to ask whether the anisotropy in the transfer and the dominantly nonlocal transfer in the $\mathrm{k}_{\mathrm{y}}$ direction are arifacts of the size of the $\mathrm{k}$ space. While it is not possible to say definitively that it is not an artifact, all evidence suggests that it is real. The wavenumber space has been varied from $13 \times 13$ modes to $41 \times 41$ modes with the nonlocal and anisotropic features becoming more pronounced in the case of a larger $k$ space, rather than the contrary. Furthermore, both the nonlocality and anisotropy are corroborated by the structure of the one-point closure equations and, to a lesser degree, the nonlinearity itself.

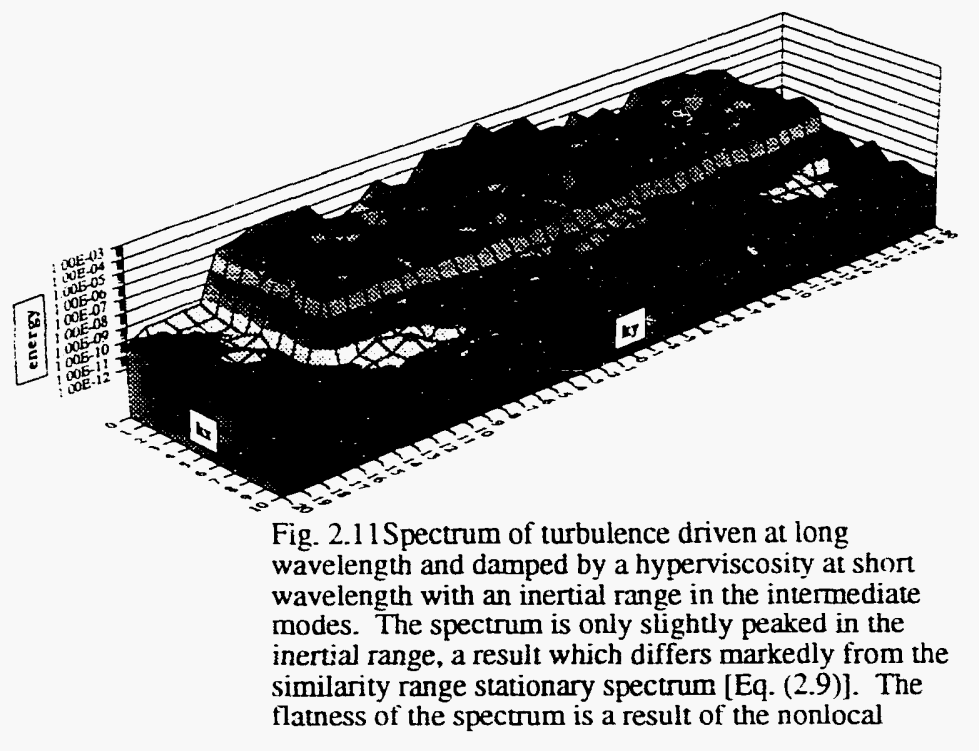




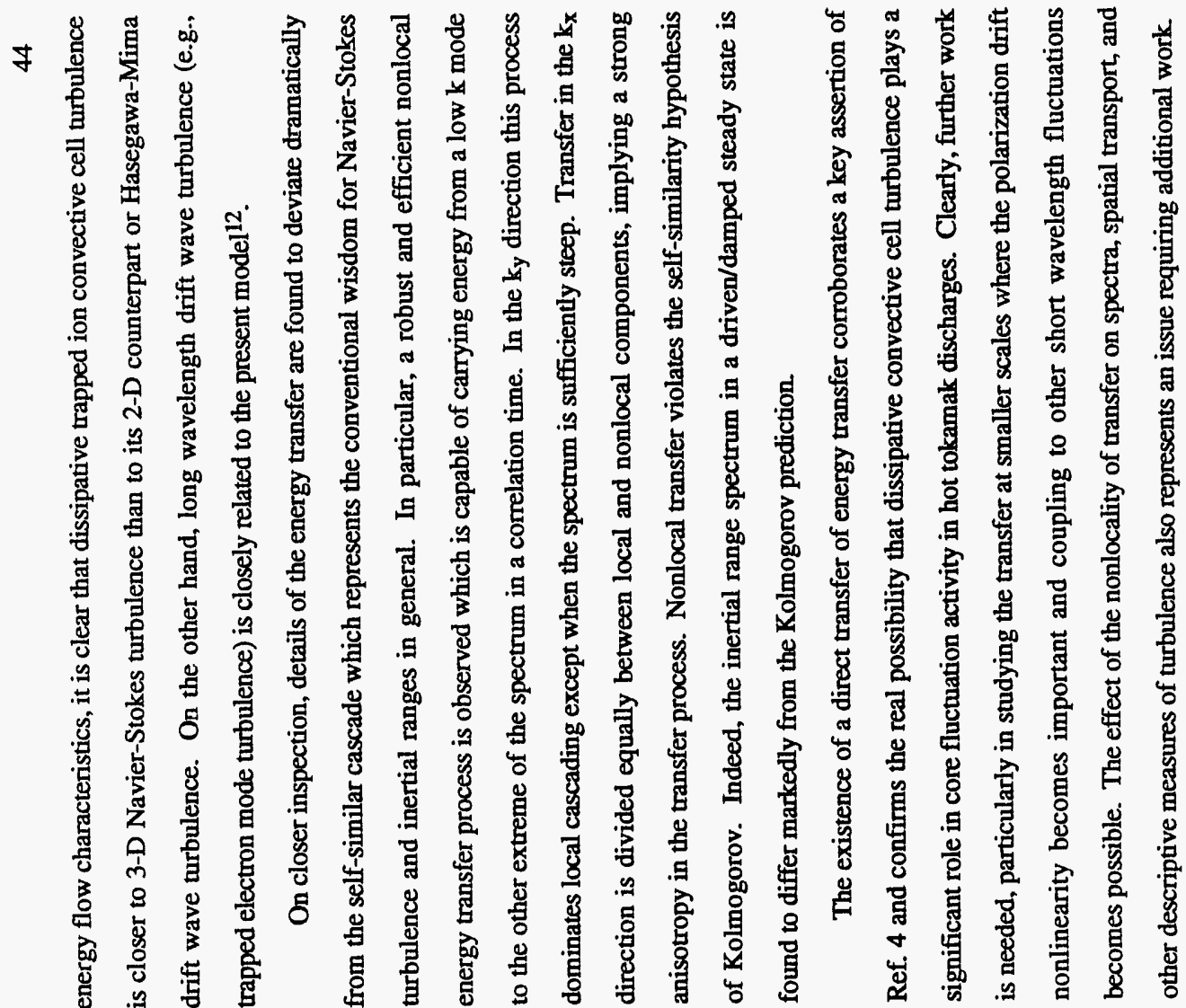

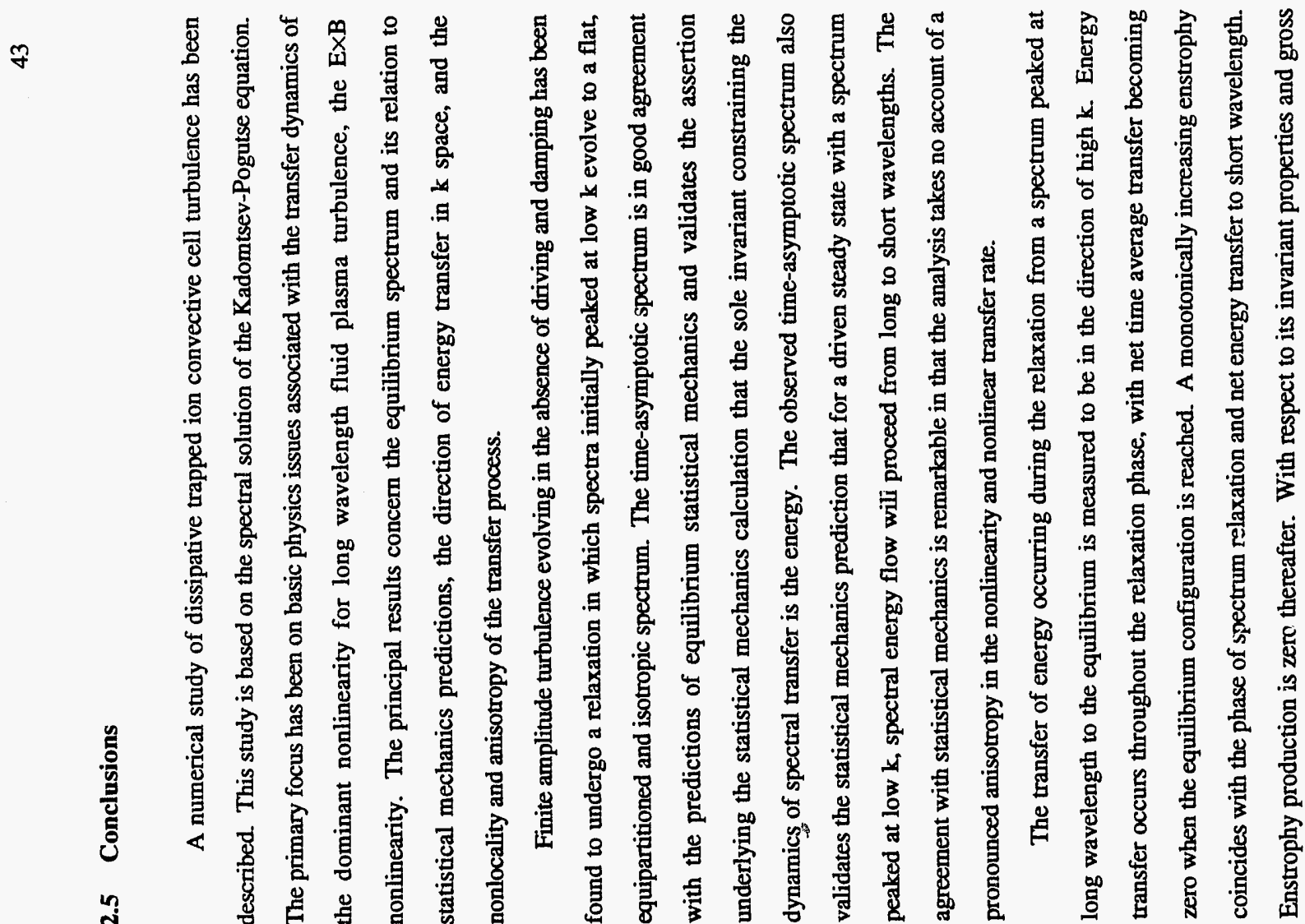




\section{References}

1. R. Saison, H.K. Wimmel, and F. Sardei, Plasma Physics 20, 1 (1978)

2. W. Horton, D.I. Choi, P.W. Terry, and D. Biskamp, Phys. Fluids 23, 590 (1980)

3. H. Biglari, P.H. Diamond, and P.W. Terry, Phys. Rev. Lett 60, 200 (1988).

4. P.H. Diamond and H. Biglari, Phys. Rev. Lett. 65, 2865 (1990).

5. R.J. Fonck, S.F. Paul, D.R. Roberts, Y.J. Kim, N. Bretz, D. Johnson, R. Nazikian, and G. Taylor, 18th European Conference of Controlled Fusion and Plasma Physics (European Physical Society, Vol. i5C, Part I, 1991) p. I-269.

6. P. Cripwell and A.E. Costley, 18th European Conference of Controlled Fusion and Plasma Physics (European Physical Society, Vol. 15C, Part I, 1991) p. I-17.

7. R.H. Kraichnan, J. Fluid Mech. 67, part 1, 155 (1975).

8. S.A. Orszag, "Lectures on the Statistical Theory of Turbulence", in Fluid Dynamics: Les Houches, Edited by R. Balin and J.-L. Peube, (Gordon and Breach, New York, 1973), p. 235.

9. F.Y. Gang, B.D. Scott, and P.H. Diamond, Phys. Fluids B 1, 1331 (1989)

10. G.G. Craddock, P.H. Diamond, and P.W. Terry, Phys. Fluids B 3, 304 (1991).

11. A. Hasegawa and K. Mima, Phys. Rev. Lett. 39, 205 (1977)

12. P.W. Terry and W. Horton, Phys. Fluids 25, 491 (1982)

G.G. Craddock, private communication.

nd, Phys. Fluids 28, 1419 (1985)

15. P.W. Terry and P.H. Diamond, in International Sherwood Fusion Conference, paper 2C13, Seattle, Washington, 1991.

\section{Chapter 3}

Improper Spectral flow from Polarization Drift Nonlinearity

\subsection{Introduction}

Spectral transfer of energy and other dynamical invariants, such as the enstrophy, or mean squared vorticity ${ }^{1-3}$ has long been considered an important aspect of turbulence. In particular, the nature and direction of spectral energy transfer has direct bearing on the way in which instability-driven turbulence is saturated, on the magnitude and shape of the spectrum, and ultimately on the nature of spatial transport produced by the turbulence.

A number of recent studies underscore the importance of spectral transfer. For example, it has recently been shown from closure theory ${ }^{4}$ and direct measurement of spectral transfer rates in numerically integrated model equations ${ }^{1}$, that dissipative trapped ion convective cell turbulence transfers energy from the long wavelengths of the driving instability to shorter wavelengths. This result has invalidated prior dogma which held that dissipative trapped ion convective cells would transfer energy to longer wavelengths, producing extremely large cell sizes and catastrophic transport. In related studies of broad band dissipative trapped electron mode turbulence 2,3 , it has been found that the spectral transfer evinces two distinct subranges at long and short wavelength extremes, separated by a highly complex intermediate subrange. In the long wavelength subrange, energy is transferred to small scales in a process that is distinctly anisotropic and nonlocal in wavenumber space. Significant production of enstrophy accompanies the transfer to shorter wavelength. In the short wavelength subrange, nonlinear transfer very nearly conserves enstrophy. The constraint of two conserved quadratic quantities (energy and enstrophy) 


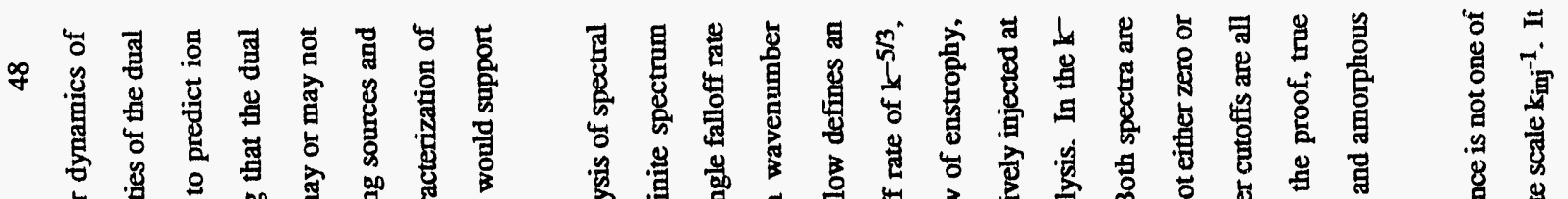

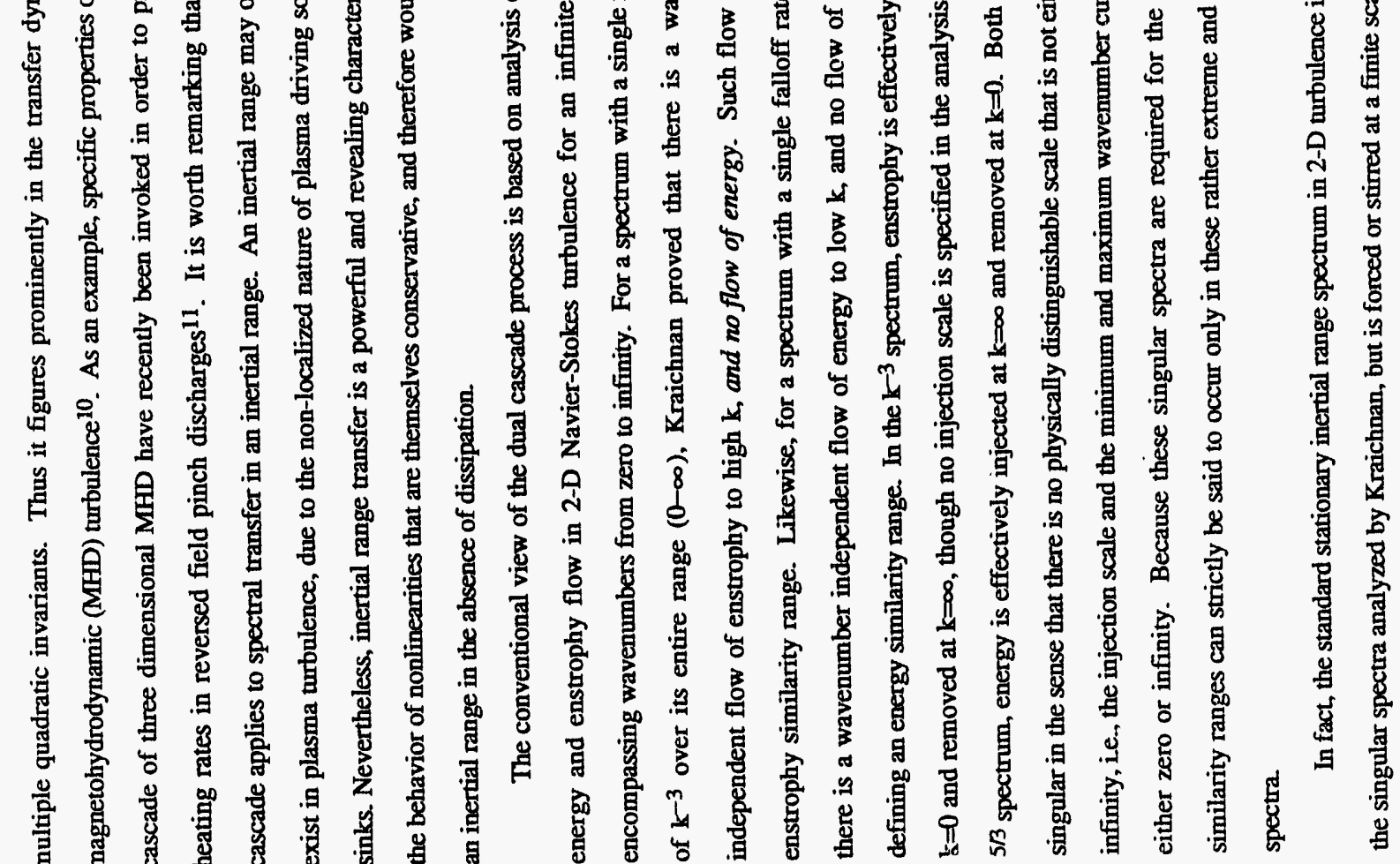

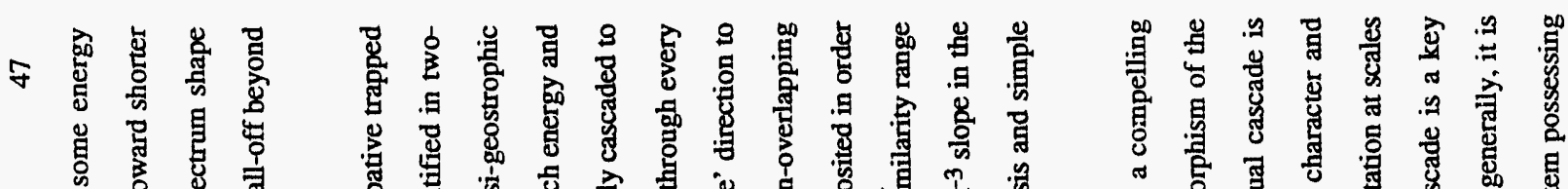

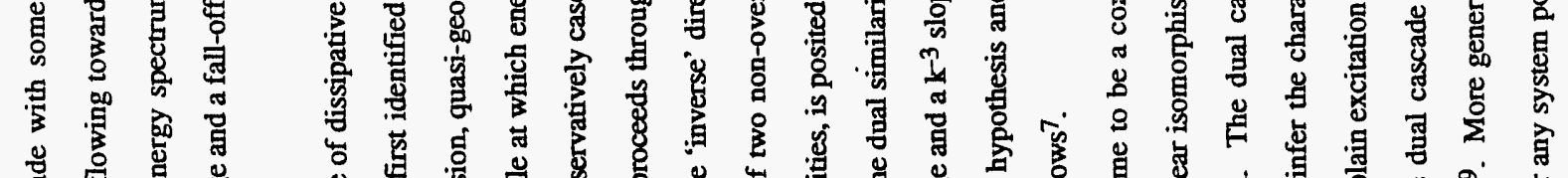

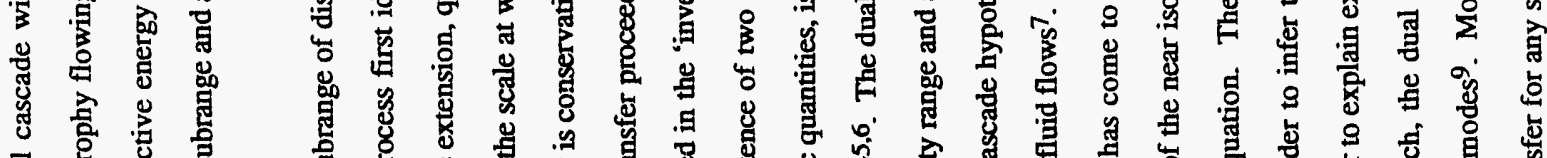

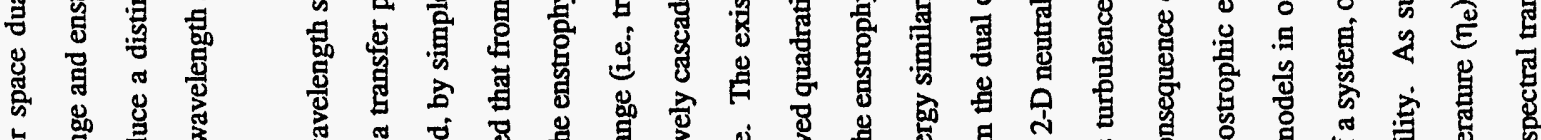

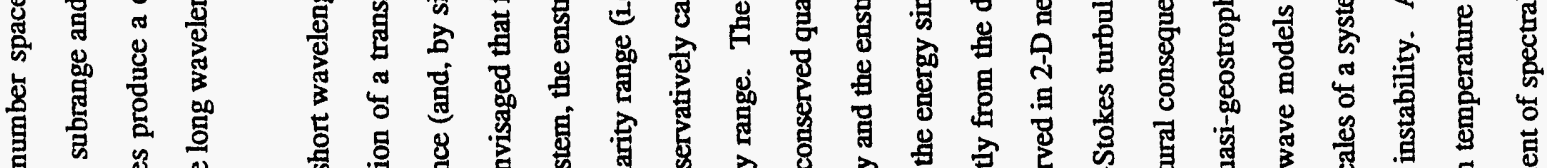

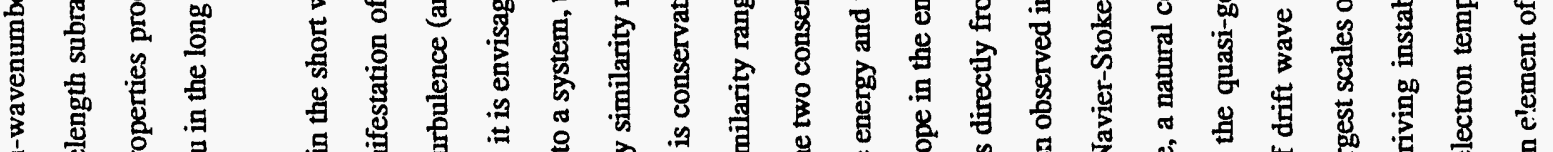

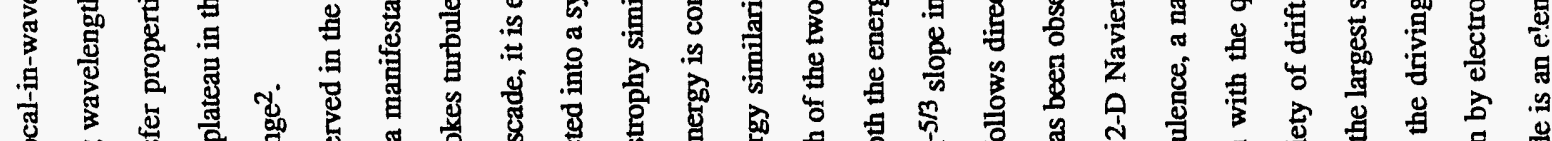

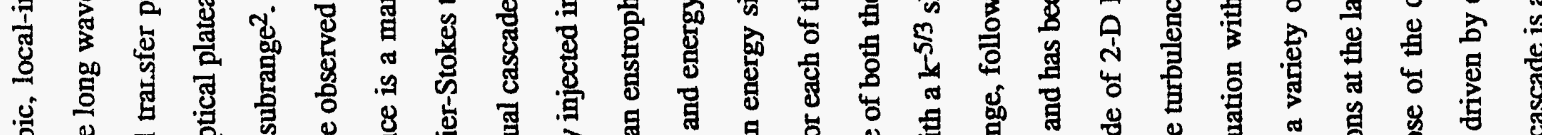

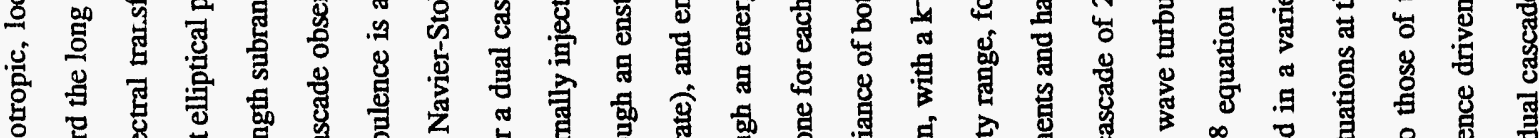

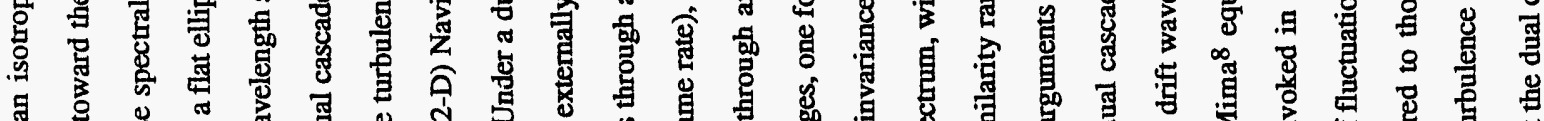
去

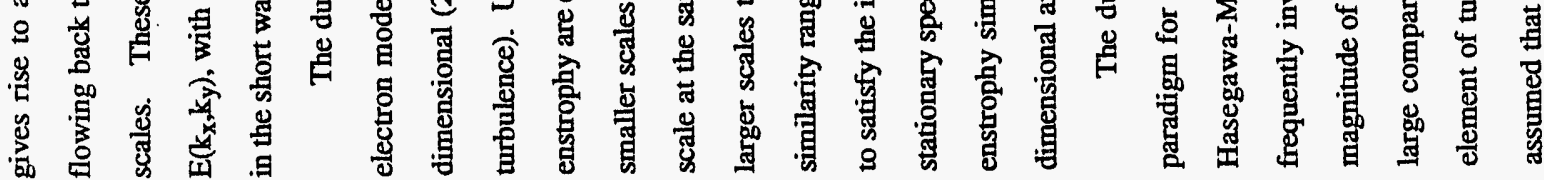



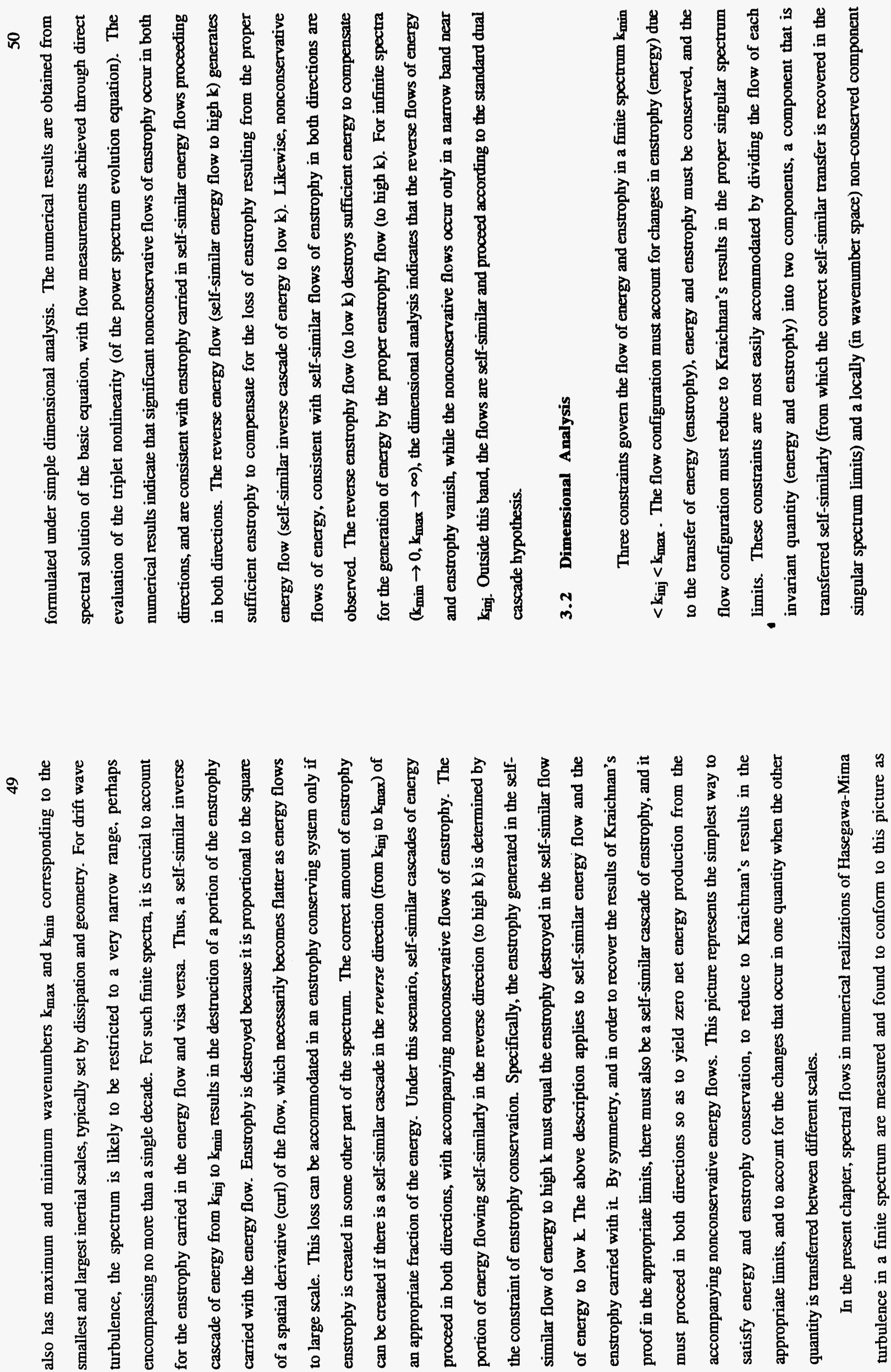
representing the energy or enstrophy carried by the self-similar flow of the other quantity. At the injection scale, all inputted energy and enstrophy must be partitioned into either of these components, so that

$E_{0}=E_{c}+E_{N}$,

$\Omega_{0}=\Omega_{c}+\Omega_{N}$,

where $E_{0}$ is the injected energy, $E_{c}$ is the amount of injected energy carried in self-similar energy flows, and $E_{N}$ is the amount of injected energy in the non-conserving energy flows representing the energy carried by the self-similar enstrophy flows. Similar definitions apply to the inputted enstrophy $\Omega_{0}$. Becanse enstrophy is mean squared vorticity, the Fourier enstrophy $\Omega(k)$ (defined as the enstrophy of a Fourier mode of wavenumber $k$ ) of any flow with Fourier energy $E(k)$ is given by $\Omega(k)=k^{2} E(k)$. Thus the partition $E_{0}=E_{c}+$ $E_{N}$, with $E_{N}$ being the portion of injected energy carried by the self-similar enstrophy cascade, implies that

$E_{N}=k_{i n j}^{-2} \Omega_{c}$

Likewise, we have

$\Omega_{\mathrm{N}}=\mathbf{k}_{\mathrm{inj}}^{2} \mathrm{E}_{\mathrm{c}}$.

Consider now the self-similar energy flow. Let $\mathrm{E}_{\mathrm{p}}$ be the energy carried in a proper energy conserving cascade from $k_{i m j}$ to $k_{\min }$. A quantity of enstrophy $E_{p} k_{i m j}{ }^{2}$ is carried in this cascade at the scale $k_{\mathrm{inj}}{ }^{-1}$, but dwindles to $E_{\mathrm{p}} k_{\min }{ }^{2}$ as the energy reaches $k_{\min }$, a consequence of the smoother gradients associated with the large scale $k_{\min }{ }^{-1}$. Obviously, the proper cascade of energy $E_{p}$ between $k_{i n j}$ and $k_{\min }$ produces a net loss of enstrophy of magnitude $E_{p}\left(k_{i m j}{ }^{2}-k_{m_{i n}}{ }^{2}\right)$. This loss is the end result of the nonconservative enstrophy flow associated with the proper energy cascade. To assure overall conservation of enstrophy, enstrophy equal to the amount lost must be generated somewhere in the spectrum. This can occur if a portion of energy $E_{r}$ cascades conservatively in the reverse sense from $k_{i n j}$ to $k_{\max }$. The steeper gradients associated with a flow of energy $E_{r}$ at a scale $k_{\max }{ }^{-1}$ results in a net production of enstrophy of magnitude $E_{r}\left(k_{\max }{ }^{2}-k_{i m j}{ }^{2}\right)$. Equating the net loss of enstrophy resulting from the proper cascade of energy with the net production of enstrophy resuling from the reverse cascade, the energies $E_{p}$ and $E_{I}$ are

$$
\begin{aligned}
& E_{p}=E_{c} \frac{\left(k_{\max }{ }^{2}-k_{\text {in }}{ }^{2}\right)}{\left(k_{\max ^{2}}-k_{\min ^{2}}\right)}=E_{c} \frac{\left(1-I^{2}\right)}{\left(1-R^{2}\right)}, \\
& E_{r}=E_{c} \frac{\left(k_{i m i}{ }^{2}-k_{\min ^{2}}\right)}{\left(k_{\max }{ }^{2}-k_{\min }{ }^{2}\right)}=E_{c} \frac{\left(R^{2}-R^{2}\right)}{\left(1-R^{2}\right)},
\end{aligned}
$$

where the total amount of conservatively cascaded energy $E_{c}=E_{l}+E_{p}$ is split into proper and reverse components, $I=k_{\text {inj }} / k_{\max }$ and $R=k_{\min } / k_{\max }$. From these expressions it is obvious that as $k_{\max } \rightarrow \infty$ with $k_{i m j}$ remaining finite, $E_{p} \rightarrow E_{c}$ and $E_{r} \rightarrow 0$, yielding a unidirectional self-similar flow to low $k$, consistent with the dual cascade hypothesis for $k_{\text {inj }}$ finite.

These arguments can be repeated for the conservative spectral flow of enstrophy. If $\Omega_{p}$ is the portion of enstrophy undergoing a proper self-similar cascade from $k_{i n j}$ to $k_{\max }$, energy carried in this flow decreases from its value $\Omega_{p} k_{i n j}{ }^{-2}$ at the scale $k_{i m j}$ to $\Omega_{p} k_{m a x}{ }^{-2}$ at $k_{\max }$, i.e., a net amount of energy $\Omega_{p}\left(k_{i n g}{ }^{-2}-k_{\max }{ }^{-2}\right)$ is lost in the conservative enstrophy transfer. Consequently, there must be a portion of enstrophy $\Omega_{r}$ reverse-cascaded to $k_{\min }$. which produces a net increase of energy $\Omega_{r}\left(k_{\min }{ }^{-2}-k_{i m j}{ }^{-2}\right)$. Equating the net loss and gain of energy in order to maintain energy conservation, the quantities of enstrophy cascaded in the proper and reverse directions are 

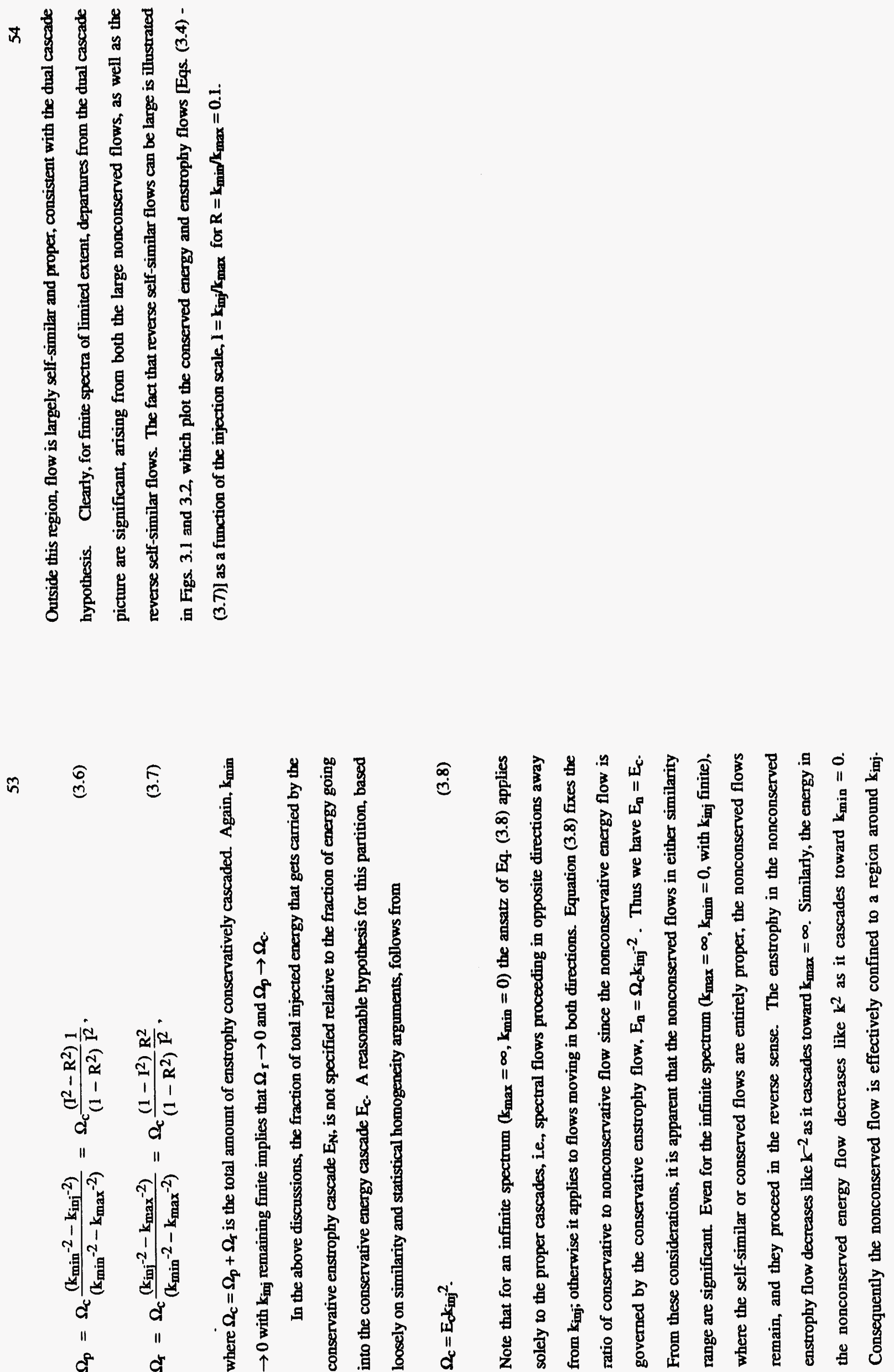


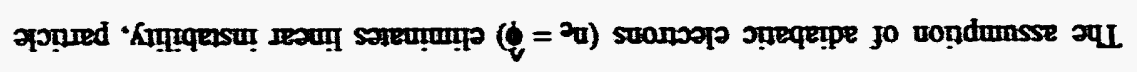

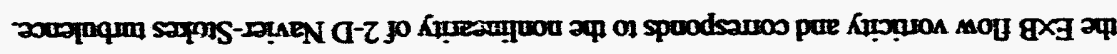

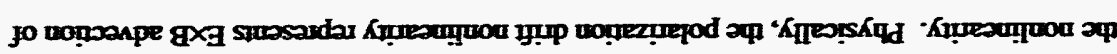

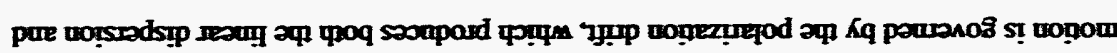

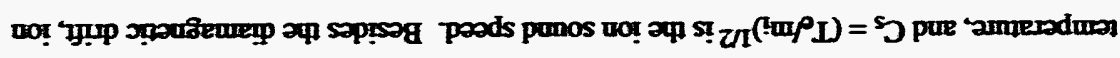

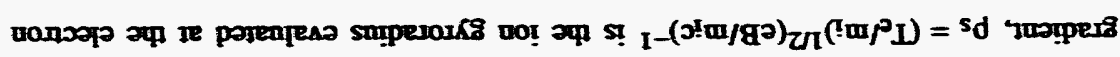

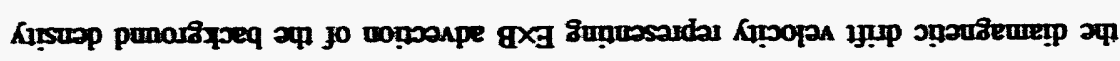

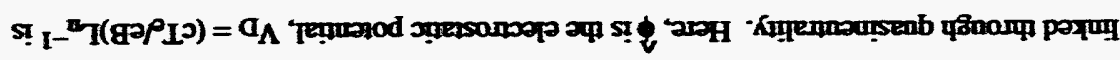

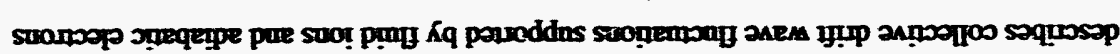

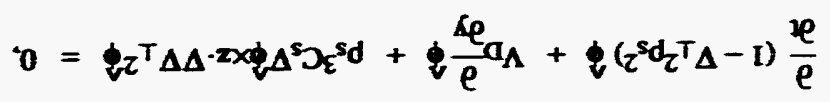

tropenbs

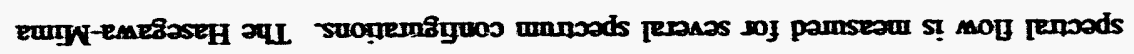

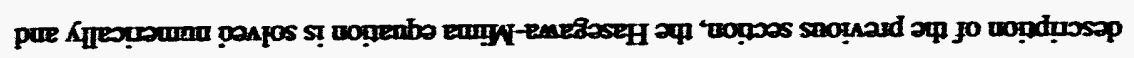



\section{Suspeur peoproun EE}

\section{amond apesses penp preputas}

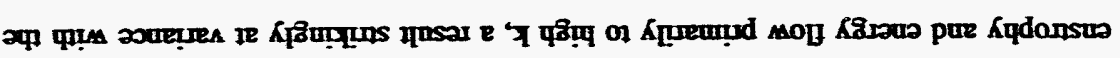

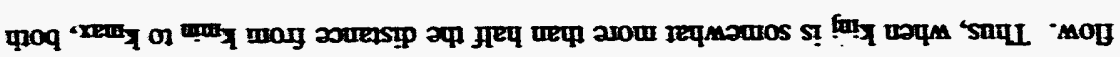

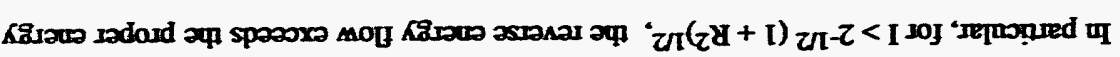

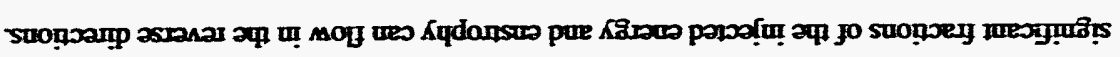

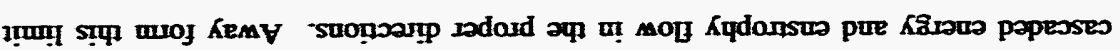

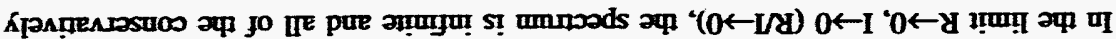

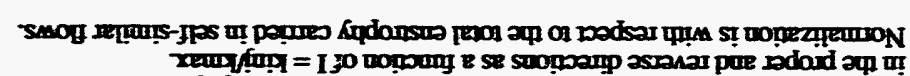

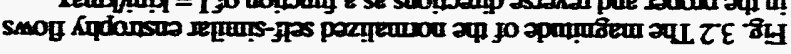

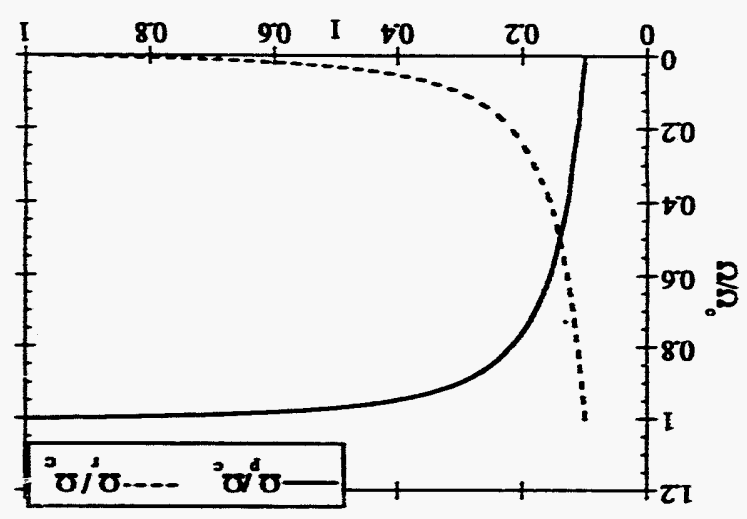

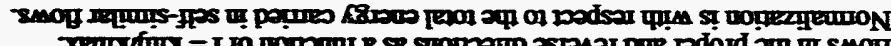

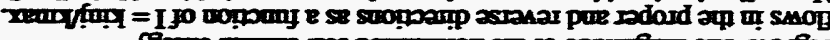

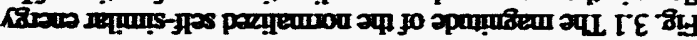

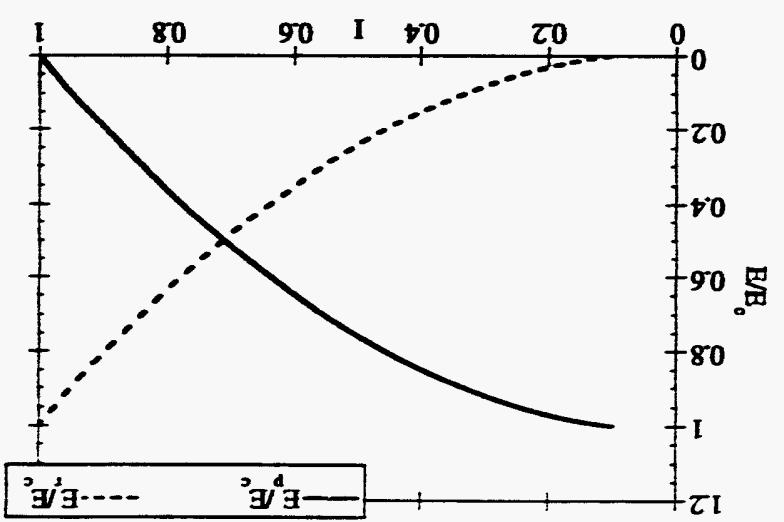



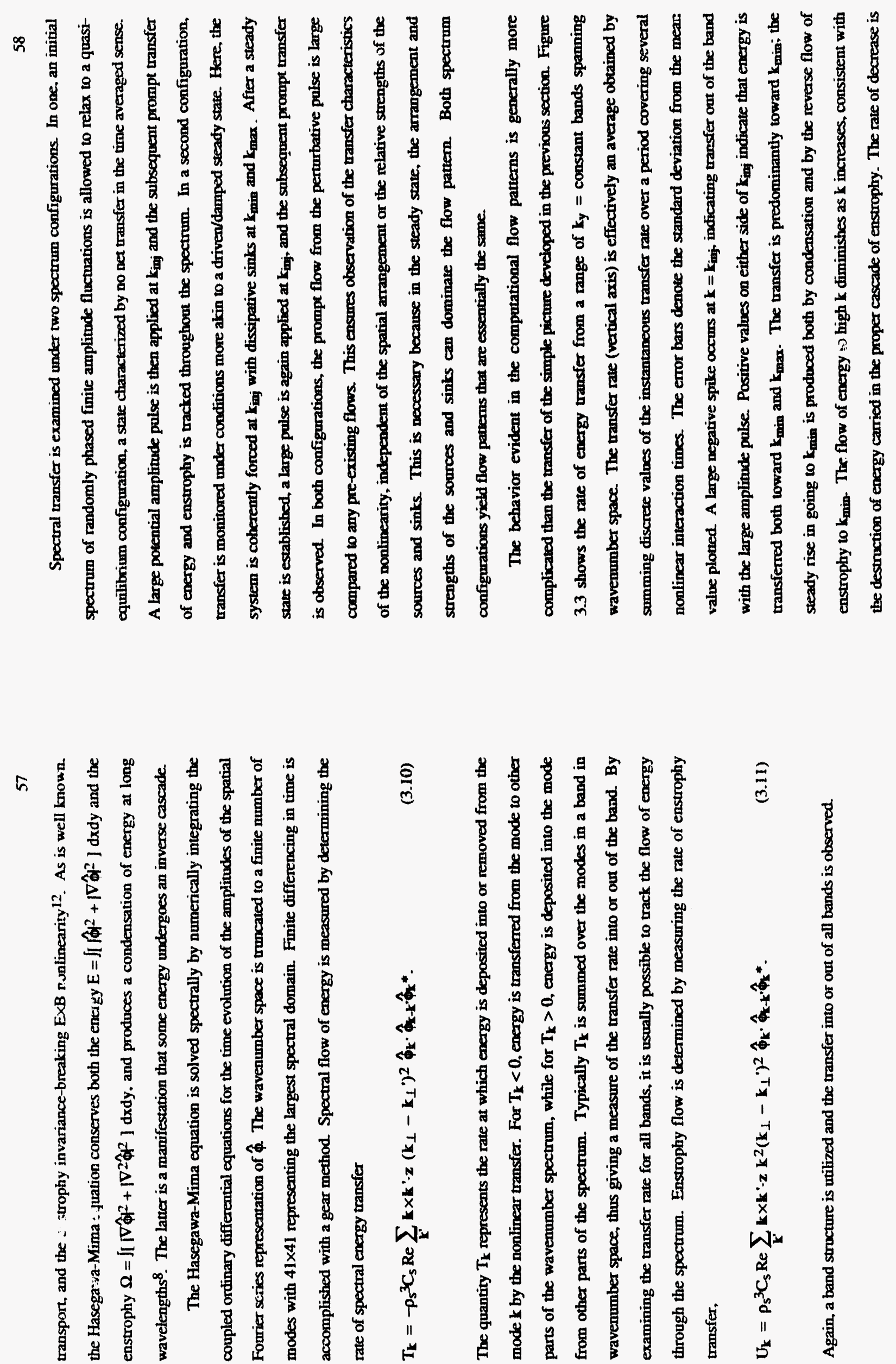
8
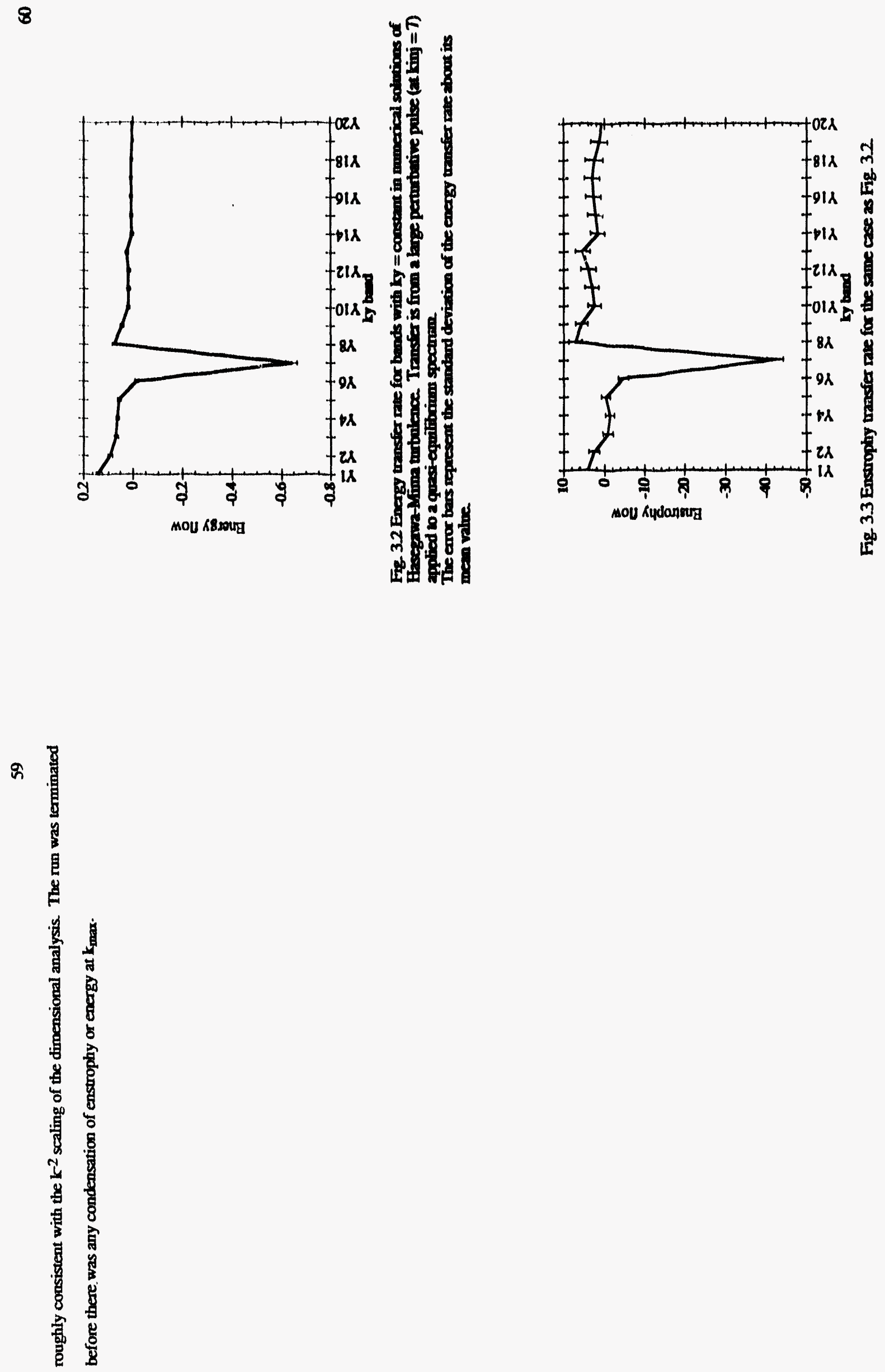
The flow pautern for enstrophy is displayed in Fig. 3.4. Again enstrophy is seen to flow from $k_{i n j}$ in both directions. Here, however, more enstrophy flows toward high $k$ than to low $\mathbf{k}$ Condensation of enstrophy at $k_{\min }$ is clear evidence of a reverse enstrophy cascade but masks the nonconservative enstrophy flow associated with the proper energy cascade. The flow of enstrophy to high $\mathbf{k}$ is roughly constant, within error bars, over the enstrophy similarity range. It is worth noting that a self-similar (steady state) cascade would produce zero net transfer into or out of any band. Here the flow is the transient response to the perturbative pulse and represems the propagation of the bulk of enstrophy in the pulse to high $k$, before condensation at $k_{\max }$.

It is likely that differences between the results of Figs. 3.3 and 3.4 and the simple relations derived in the previous section stem both from limitations in the numerical wort and the extreme simplicity of the dimensional analysis. In particular, the spectrum configuration is of ten arranged with $k_{\text {ing }}$ as the geometric mean of $k_{\max }$ and $k_{\min }$ Among other things, this choice makes the fractional proper cascaded encrgies and enstrophies comparable $\left(\mathrm{E}_{\mathrm{p}} / \mathrm{E}_{\mathrm{c}}=\Omega_{\mathrm{p}} / \Omega_{c}\right)$. Moreover, there is some evidence that this scale represents a natural break point for dual cascades in relaxing spectra with no forcing or perturbative pulse. Under sach circumstances, $k_{i n j}$ is typically closer to $k_{\text {min }}$ than it is to $k_{\text {max }}$ Consequently, there is considerable energy condensation at $k_{\min }$ before the transfer to high $\mathbf{k}$ reaches $k_{\max }$. Becanse condensation produces spectrum ctanges that then affect transfer, it becomes difficult to track the upward transfer all the way to $k_{\max }$ once condensation has begun at $k_{\min }$ A second limitation results from the amalysis of transfer into or out of bands of constant $k_{x}$ or $k_{y}$. This band structure permits the examination of anisotropies of the transfer rate. However, for the Hasegawa-Mima equation, the transfer is found to be isotropic, in which case the constant $k_{x}$ or $k_{y}$ band structure bas the unwanted effect of potentially distorting measuremem of transfer. For example, self-similar transfer of energy within a $k_{x}=$ constant band (from large $k_{y}$ to small $k_{y}$ ) can decrease the enstrophy of the band, independent of the transfer occurring between bands. Finally, the observed flow patterns are affected by random fluctwations of the nonlinear imberaction. Even though the flow parterns are time averaged, some random component remains after averaging.

Each of the above difficulties suggeses improvements for future computional work However, there is considerable agreement between the simple model and the resalts of this imperfect cumputational amalysis. Cerainly, the measured flow panerns are considerably ahered from those envisioned in the standard dmal cascade hypothesis Energy and enstrophy are uransferred away from $k_{\text {mij }}$ in both firections. Moreover, there is evidence that the flow in a given direction away from $k_{\text {inj }}$ is not completely self-similar. Also, runs with inctial ranges varied by over a factor of 3 indicate that the flow panerns tend toward the standard dual cascade configuration as the spectal range increases, i.e, the magnitude of the reverse (conservative) flows decreases relative to that of the proper flows. 
3.4 Conctusions and Discussion

The notion that energy and enstrophy respectively undergo self-similar cascades to long and short wavelengths in tubulence conserving these quamities has been shown to be appropriate only for infinize spectra $\left(k_{\min } \rightarrow 0, k_{\max } \rightarrow \infty\right)$ away from the injection scale. Because enstrophy (energy) is carried in wavenumber space by a self-similar energy flow (enstrophy flow) and increases or decreases depending on the direction of the flow, injected energy and enstrophy must flow from the imjection scale in both directions. The energy lost in the proper self-similar cascade of enstrophy to high $\mathbf{k}$ is then compensated by the energy gained in a reverse self-simitar cascade of enstrophy to low $k$ A simitar stmement applies to enstrophy lost and gained from proper and reverse energy cascades to low and high $k$ respectively. These constraints have been incorporated into simple scaling expressions from which the magnitude of proper, reverse, and the non self-simitar flows are obtained. The standard dual cascade results are recovered from these expressions in the fimit $k$ min $\rightarrow 0$ and $k_{\max } \rightarrow \infty$. These expressions therefore yield the dominant flow patern of the infinite spectrum, a result ofien inferred from staistical mechnoics arguments.

Large reverse energy flows are predicted when $k_{\text {inj }}$ is near $k$ max. This case is instructive in reference to $\eta_{e}$ turbulence? ${ }^{9}$, where it has been asserted that there is an inverse cascade of energy to scales given by clope (where $\omega_{p e}$ is the electron plasma frequency) from the smaller scales of collective exciration at $p_{e}$ (the electron gyroradius). Writh driving already at very small scales, in is fikely that discipation cocurs at scales only stightly above Pes in which case Fig. 3.1 suggests that the dominant energy transfer would be toward short wavelengtins, not the longer ciwpe scales. Because enstrophy also flows to high $\mathbf{k}$, the dominant non self-similar energy flow would also be toward high $k$

This type of consideration clearly demonstrates that the practice of invoking a standard dual cascade for spectra with an inertial range bounded between limits not widely separated in waverumber space may not be vafid. Even if damping is restricted to a region Ouside maximum and minimum cutoffs, the location of the scale at which fluctuations are excited within the region will play a significant role in the firection of energy flow. Moreover, because sources and sinks may in fact be distributed, with no true inctivil range, decemining the $k$ space flow ultimately requires a knowledge of the spectrum. This, in turn, requires solution of the appropriate two-point equations, taking account of both the distributions of sources and sinks and the specton properties of the nonfineoritiex. 


\section{References}

1. D.E Newman. P.W. Terry, and P.H. Diamond. Phys. Fluids B 4, 599 (1992)

2. D.E Newman. P.W. Terry, P.H. Diamond, and Y.-M. Liang, Phys. Fluids B. 3. Y.M Liang, P.H. Diamond X.H. Wang. DE Newman, and P.W. Terry. Phys.
Fluids B.

P.H. Diamond and H. Biglari, Phys. Rev. Lett 65, 2865 (1990).

R.H. Kraichnan, Phys. Fluids 10, 1417 (1967).

C.E. Leith, Phys. Fluids 11, 671 (1968).

M. Gharib and P. Derango, Physica D 37, 406 (1989)

A. Hasegawa and K. Mima, Phys. Rev. Lett 39, 265 (1977).

W. Horton, B.-G. Hong, T. Tajima, and N. Bekki, Comments Plasma Phys. Controlled Fusion 13, 207 (1990).

10. A. Pouquet, U. Frisch, and J. Leorat, J. Fluid Mech. 77, 321 (1976)

11. N. Mattor, P.W. Terry, and S.C. Prager, Comments Plasma Phys. Controlled Fusion 15, 65 (1992).

12. P.W. Terry and W. Horton, Phys. of Fluids 25, 491 (1982)

\section{Chapter 4 \\ Dynamies of Coupled Nonlinearities}

4.1 Introduction

Recent analytical work indicates that trapped ion convective cell turbulence is a viable candidate for long wavelength fluctuation activity in hot, auxiliary heated core plasmas1. Beyond its promise as a core fluctuation and transpont model, however, trapped ion convective cell turbalence provides an instructive paradigm for long wavelength turbulence and the spectral transfer properties that ultimately govern its saturation, spectral distribution of energy, and transport ${ }^{1.2}$. Its usefulness as a paradigm follows in part from the fact that trapped ion convective cell turbulence can be described by a one-field fluid model, e.g, the Kadomtsev-Pogutse equation, thus allowing a succinct and transparent representation of the nonlinear mode coupling process. More importantly, a generalization of the Kadomtsev-Pogutse model provides a mode coupling representation with two nonlinearities, the EXB and polarization drift nonlinearities, and is therefore generic to drift wave type fluctuations. For example, the ExB and polarization drift nonlinearities are the nonlinearities that appear in the one-fluid description (Terry-Horton) of turbulence associated with dissiparive trapped electron and universal modes?

Chapter 2 presented theoretical and computational analysis of purely trapped ion convective cell turbulence that has focused on the long wavelength regime, where nonlinear transfer is dominated by the EXB nonlincarity. These studies demonstrated that the transfer of energy in wavenumber space is directed toward short wavelength. The direct cascade of energy is possible because the conservative transfer of energy is not subject to any additional constraints, i.e, besides the energy, the ExB nonlinearity conserves no other 
nontrivial quadratic quantity. In addition to a direct cascade of energy, the EXB nonlinearity produces energy transfer which is anisotropic ${ }^{2}$ and highly nonlocal in wavenumber space ${ }^{1,2}$. The occurrence of strong nonlocal transfer is a marked departure from the selfsimilar cascade dynamics inherent in the scaling arguments of Kolmogorov. The anisotropy of transfer follows from an absence of symmetry induced by the $k_{y}$ dependence of the nonadiabatic electron response in the EXB nonlinearity. The anisotropy manifests itself as a transfer which is strongly nonlocal in the $\mathbf{k}_{\mathbf{y}}$ direction (cross-field direction perpendicular to the density gradient) but characterized by comparable local and nonlocal transfer rates in the $\mathbf{k}_{\mathbf{x}}$ (gradient) direction.

The long waveiength regime of trapped ion convective cell turbulence contrasts strongly with a more familiar drift wave turbulence paradigm, the Hasegawa-Mima model ${ }^{8}$. In the Hasegawa-Mima equation, neglect of nonadiabatic electron dynamics eliminates the EXB nonlinearity and leaves the polarization drift nonlinearity (normally subdominant to the E $\times B$ nonlinearity in the long wavelength limit) as the sole spectral transfer mechanism. While the neglect of nonadiabatic electron dynamics excludes the possibility of describing either instability or transport, it does produce a model which is nearly isomorphic to the quasi-geostrophic equation. Accordingly, two dynamical invariants are admitted by the polarization drift nonlinearity, ie., the energy and the enstrophy, or mean squared vorticity. In order to satisfy both constraints, a dual cascade process is required with the energy undergoing an inverse cascade or transfer to long wavelength, as in two-dimensional Navier-Stokes turbulence. The cascade dynamics of the Hasegawa-Mira equation are representative of the conventional view of spectral energy transfer in 2-D plasma turbulence. In particular, the notions of the inverse cascade and local transfer in wavenumber space are pervasive in heuristic descriptions of saturation and spectral dynamics.

With nonadiabatic electrons, spectral transfer is affected by both the ExB and polarization drift nonlinearities, and enstrophy conservation, in a strict sense, is broken.
However, the polarization drift nonlinearity involves a higher derivative of the fluctuating potential than the EXB nonlinearity. Consequently, the magnitude of the polarization drift nonlinearity becomes much larger than the magnitude of the EXB nonlinearity at short wavelengths, while the opposite holds at long wavelengths. It would seem reasonable, therefore, to predict the existence of spectral ranges in the short and long wavelength limits of the spectrum in which the transfer dynamics is dominated by one or the other of the nonlinearities. This wonld mean there is a spectral range at long wavelengths where energy is transferred to smaller scales and in which the total enstrophy within the range evolves on the timescale of the nonlinear interaction. There would also be a range at short wavelengths where energy is transferred to larger scales and enstrophy is approximately conserved on the timescale of nonimear interactions. One of the primary aims of this chapter is the testing of this hypothesis and the characterization of spectral transfer properties in both the intermediate range where the two nonlinearities play an active role and over the larger spectrum as a whole. Such a study is of direct relevance to trapped ion and electron turbulence because the firect transfer of energy generated at long wavelengths ( $\omega<a_{b i}$ ) will inevitably carry energy to a spectrum region where the polarization drift nonlinearity is important, ie. the dissipative trapped electron mode (DTEM) regime where $\omega_{b e}>\omega_{b} \omega_{b i}$.

It is also of considerable interest to examine the interplay of the two disparate transfer processes in terms of the locality and anisotropy of spectral transfer. In particular, as mentioned above, the EXB nonlinearity, acting alone, is known to produce transfer that is both nonlocal and anisotropic1,2. On the other hand, the polarization drift nonlinearity is isotropic in form, and by analogy with the two-dimensional Navier-Stokes equation, should transfer energy locally in wavenumber space ${ }^{9}$, consistent with the notion of a Kolmogorovtype similarity range and cascade. 
In this chapter, broad-band nonlinear transfer dynamics is examined for dissipative trapped electron mode turbulence (DTEM), including both the ExB and polarization drift nonlinearities. This work is an outgrowth of a previous study which considered only the $\mathrm{E} \times \mathrm{B}$ nonlinearity ${ }^{2}$, and is based on numerical solution of a model equation utilizing direct measurement of local and nonlocal spectral transfer rates. The computational work is accomplished with a spectral code containing up to $41 \times 41$ modes. The simulations examine two general situations: 1) the relaxation of the spectral energy distribution from an initial finite amplitude configuration in the absence of driving and damping; or 2) the evolution and saturation of the spectrum starting from infinitesimal amplitudes driven by unstable modes at low wavenumber and damped modes at high wavenumber. The first situation permits measurement of the nonlinear transfer rate independent of any particular wavenumber space configuration of sources and sinks, consistent with a chosen general spectrum shape. This situation also enables comparison of the stationary spectrum achieved by relaxation from an initial state with the predictions of equilibrium statistical mechanics. The second situation addresses the transfer characteristic of the most likely arrangement of sources and sinks. In this case, the net energy transfer in a saturated state (assuming one occurs) is not in question, since it will necessarily proceed from source to sink. Rather, these studies will determine the spectrum shape and examine nonlinear transfer in subranges of wavenumber space, focusing on the issues of direction, locality, and isotropy.

A striking visualization of the energy transfer in the saturated state may be produced by subjecting the saturated spectrum to a large, localized (in wavenumber space) perturbation and observing the subsequent relaxation of the spectrum to its original stationary configuration. Using this technique it is possible to determine the direction, locality, and isotropy of transfer is various subranges of the spectrum. This technique is the spectral analog of perturbative transport studies using modulated gas feed, heat pulse propagation, etc. to infer the locality, direction, and magnitude of spatial transport. As such, it represents a reasonably accessible means of examining spectral transfer and cascades in experiment. Tests of this technique in simulated turbulence are reported herein.

The principal results of this chapter are now summarized. It is found that the polarization drift nonlinearity, acting alone, produces transfer that is both isotropic and local. Both properties follow directly from symmetries of the nonlinearity that are not present in the pure Kadomtsev-Pogutse model. With both nonlinearities acting simultaneously, subranges exist in which the transfer is virtually indistinguishable from the transfer of the dominant nonlinearity, if acting alone. Enstrophy generation is shown to be an effective indicator of the dominance of one nonlinearity over the other in these cases. If the maximum and minimum wavenumbers restrict the spectrum to a range where the $E \times B$ nonlinearity dominates throughout, enstrophy production is significant and increases on a timescale of a few eddy turnover times. Energy is transferred to high $\mathbf{k}$ in an anisotropic and nonlocal process characteristic of cases in which the polarization drift nonlinearity is entirely absent If the spectrum is fixed to a range in which the polarization drift nonlinearity dominates throughout, enstrophy production is weak, with an e-folding time much larger than the eddy turnover time. Here a dual cascade is evident and transfer is isotropic and local. A third subrange exists and is accessed by determining the maximum and minimum wavenumbers so that both nonlinearities are roughly comparable over most of the spectrum (i.e., neither dominates). In this subrange, enstrophy production is moderate. Spectral transfer tends to be isotropic but retains a strong nonlocal component. In this situation, the local transfer develops an anisotropy which offsets the anisotropy of the nonlocal flow. When undriven, undamped turbulence evolves from an initial spectrum peaked at low $\mathrm{k}$, the spectrum relaxes to a time-asymptotic state that remains peaked at long wavelength but has two subranges with distinct spectral falloff rates. The subranges separate at the point where the two nonlinearities are equal, with a slightly steeper slope in the high $\mathrm{k}$ range than in the low $\mathrm{k}$ range. Energy flow is toward higher $\mathrm{k}$ in the low $\mathrm{k}$ 
subrange, with the converse true in the high $\mathbf{k}$ subrange. Present limits on $\mathbf{k}$-space resolution make it difficult to extend this subrange to sufficiently large and small wavenumber to allow each nonlinearity to dominate at the extremes of the spectrum. It is anticipated that a larger subrange would accentuate, in each of its extremes, both the spectrum falloff disparity, and the differences in energy transfer direction.

For turbulence driven by unstable modes at long wavelength and damped by a hyperviscous damping at small wavelength, there is a noticeable difference in the wavenumber spectra which occur when either of the nonlinearities is acting alone and the spectrum which occurs in the subrange where when both nonlinearities are present and comparable. Whereas the spectrum of the ExB nonlinearity tends to be flat ${ }^{2}$, it falls off toward high $\mathbf{k}$ with both nonlinearities. There is a discernible change in the falloff rate at the wavenumber where the two nonlinearities are equal, with a flatter falloff in the longer wavelength part of the spectrum. Distinct differences in the transfer rate are observed. In interpreting the transfer diagnostics, it appears that transfer is more sensitive to the crosscoupling of the nonlinearities than is the spectrum. Consequently, transfer behavior in either subrange with the unmodified signature of the dominant nonlinearity is more difficult to discern in the relatively small wavenumber space of the present simulations. Nevertheless, the following statements represent the qualitative behavior of the transfer with both nonlinearities in the steady state. In the ExB subrange, transfer is nonlocal. Above a critical wavenumber (related to the wavenumber where the two nonlinearities are equal), nonlocal transfer becomes weak relative to the total transfer. Thus, there is a polarization subrange with local transfer. A dual cascade is evident in this region of $k$ space and accounts for the change in spectral index in crossing from one subrange to the other.

A novel and potentially important aspect of the interplay between the two nonlinearities is evident in the frequency spectra of individual modes. In the regime where both nonlinearities are comparable, a large shift of the frequency spectrum peak is observed.
For shorter wavelength modes, the shift is to higher frequency and can be many times the diamagnetic frequency. If either nonlinearity is absent, or contributes only weakly to the transfer dynamics, the shift is small. Theoretically, a spectrum shift is found in the renormalized response function 10 . This shift arises from the cross-coupling of the nonlinearities through the driven fluctuations. Since the EXB nonlinearity has one fewer spatial derivatives than the polarization drift nonlinearity, the cross-coupling term is $90^{\circ}$ out of phase with the eddy damping decrement, and thus enters as a shift in the frequency spectrum peak (as opposed to a broadening). The occurrence of a frequency shift has a potentially significant impact on stability and transport ${ }^{10}$, as well as the interpretation of fluctuation measurements. A derivation of the frequency shift and its effect on nonlinear stability, and therefore on the spectrum, is explored in Ref. 10. The self-consistent effect of the shift on mode fluctuation levels cannot be described by the present computational model and will be addressed in future work

The chapter is organized as follows. The model and its properties are presented and discussed in the next section. Section III describes the spectral transfer properties. To facilitate interpretation of the results, a subsection examines the locality and isotropy of transfer of the polarization drift nonlinearity. In Sec. IV, measurements of the frequency spectrum are presented and discussed. The conclusions are given in Sec. V. 


\subsection{Basic Model and Properties}

In this section the basic model is presented and the dynamical coupling of the two nonlinearities is analyzed from the perspective of the mathematical structure of the model. This analysis examines integral invariants, symmetries in the structure of the nonlinearities, and the renormalized mode equations.

The model utilized for this study is a trapped particle fluid equation. This model couples the laminar dynamics of collisional trapped electrons with hydrodynamic ions through the quasineutrality condition. The derivation of this equation and a discussion of its details and limitations have been presented elsewhere $1,2,7,10$. In previous work, the polarization drift nonlinearity was neglected by considering only the long wavelength extreme of the spectrum ${ }^{1,2}$. Here, the polarization drift nonlinearity is included, yielding the model equation:

$$
\begin{aligned}
& \frac{\partial \tilde{\mathbf{n}}}{\partial t}+D \frac{\partial^{2} \tilde{\mathbf{n}}}{\partial y^{2}}+V_{D} \frac{\partial \tilde{\mathbf{n}}}{\partial y}+v_{i} \tilde{\mathbf{n}}-L_{\mathbf{n}} D \nabla \frac{\partial \tilde{\mathbf{n}}}{\partial y} \times z \cdot \nabla \tilde{\mathbf{n}} \\
& +\rho_{s} C_{s} \nabla \tilde{n} \times z \cdot \nabla \rho_{s}^{2} \nabla^{2} \tilde{n}+\mu \nabla^{4} \tilde{n}=0,
\end{aligned}
$$

where $\tilde{n}$ is the fluctuating ion density, $V_{D}=\left(c_{d} / e B\right) L_{n}^{-1}$ is the diamagnetic drift velocity, $D$ $=\varepsilon^{1 / 2} V_{D}{ }^{2}\left(1+3 \eta_{d} / 2\right) / v_{\text {eff,e }}$ is a negative diffusivity describing the destabilization of DTEM modes by electron collisions, $\varepsilon$ is the trapped electron fraction, $\eta_{e}=\mathrm{d} l n \mathrm{~T} / \mathrm{d} l n n$ is the electron temperature gradient parameter, $v_{i}$ models long wavelength collisional damping, $v_{\mathrm{eff}, \mathrm{e}}=$ $v_{d} \in \varepsilon, \mu$ is the coefficient of the hyper-viscosity introduced to model strong damping at high $k, L_{n}$ is the density gradient scale length, $\rho_{s}=\left(c T_{e} / e B\right) / C_{s}$ is the ion gyroradius evaluated at the electron temperature, and $\mathrm{C}_{\mathrm{S}}=\left(\mathrm{T}_{\mathrm{e}} / \mathrm{m}_{\mathrm{j}}\right)^{1 / 2}$ is the ion sound speed. In keeping with the emphasis of the present chapter on nonlinear transfer effects, the linear polarization drift term responsible for linear dispersion has not been included in Eq. (4.1). This allows significant savings in computation time. Inclusion of this term introduces straight-forward modifications of subsequent equations and relations (for example, the energy and enstrophy defined below are modified when dispersion is included ${ }^{10}$ ). However, the basic mechanisms governing transfer and the concepts describing this process are unaltered. Indeed, numerical solutions with and without the linear polarization term included were found to be qualitatively the same for the spectral ranges studied herein.

The first nonlinearity appearing in Eq. (4.1) is the ExB nonlinearity, arising from $v_{E} \cdot \nabla \tilde{n}_{i}$, where $v_{E}=-\left(c / B_{0}\right) \nabla \phi \times z$ is the $E \times B$ drift. The second nonlinearity is the polarization drift nonlinearity, and arises from $n_{0} \nabla \cdot v_{p}{ }^{(1)}$, where $v_{p}^{(1)}=B_{0}{ }^{-1}$ $\left(\mathrm{m}_{\mathrm{i}} \mathrm{c} / \mathrm{e}\right) \mathbf{z} \times \mathbf{v}_{\mathrm{E}} \cdot \nabla v_{\mathrm{E}}$ is the nonlinear polarization drift. The EXB nonlinearity requires a nonadiabatic electron response (provided by the trapped electrons), whereas the polarization drift nonlinearity derives from the ion polarization drift

From Eq. (4.1), it is apparent that, by virtue of its additional spatial derivative, the polarization drift nonlinearity dominates the EXB nonlinearity at very short wavelengths. The converse holds at long wavelengths. The nominal crossover point is given by the wavenumber at which the two nonlinearities are equal. Assuming rough isotropy, so that $\nabla_{\perp} \approx \partial / \partial y$, this wavenumber is given by $k \rho_{s} \approx \delta=C_{s} / L_{n} v_{\text {eff,e }} \equiv k_{0} \rho_{s}$. Because the nonlinearities are characterized, not by a single spatial scale, but by a triad interaction consisting of three waves of differing wavelengths, it is more realistic to identify a region, centered about the crossover wavenumber, in which the two nonlinearities are comparable, rather than to speak of a single wavenumber at which the two are equal.

Due to the presence of the ExB nonlinearity, a single quadratic invariant, the energy, is admitted by Eq. (4.1) in the absence of driving and damping, i.e., 
$\frac{1}{2} \frac{\partial}{\partial t} \int \tilde{n}^{2} d^{2} x=D \int\left(\frac{\partial \tilde{n}}{\partial y}\right)^{2} d^{2} x-v_{e f f, i} \int \tilde{n}^{2} d^{2} x-\mu \int\left(\nabla^{2} \tilde{\mathbf{n}}\right)^{2} d^{2} x$,

where $E=\int \tilde{n}^{2} d^{2} x$ is the energy and the terms on the right hand side represent the dissipative source (inverse electron damping) and sinks (ion-ion collisions and hyperviscosity). Only if the $\mathrm{E} \times \mathrm{B}$ nonlinearity is absent, is there a second invariant, the enstrophy, defined by $\Omega=\int|\nabla \tilde{n}|^{2} d^{2} x$. From Eq. (4.1), the enstrophy evolution is given by

$\frac{1}{2} \frac{\partial}{\partial t} \int|\nabla \tilde{n}|^{2} d^{2} x-D \int\left|\nabla \frac{\partial \tilde{n}}{\partial y}\right|^{2} d^{2} x+v_{e f f, i} \int|\nabla \tilde{n}|^{2} d^{2} x+\mu \int\left(\nabla^{3} \tilde{\mathbf{n}}\right)^{2} d^{2} x$

$$
=-L_{\mathbf{n}} \mathbf{D} \int \nabla^{2} \tilde{\mathbf{n}} \nabla \frac{\partial \tilde{\mathbf{n}}}{\partial \mathbf{y}} \times \mathbf{z} \cdot \nabla \tilde{\mathbf{n}} \mathrm{d}^{2} \mathbf{x}
$$

The term on the right hand side escribes the generation or destruction of enstrophy associated with the conservative transfer of energy by the $\mathrm{E} \times \mathrm{B}$ nonlinearity. As previously indicated, the $\mathrm{E} \times \mathrm{B}$ nonlinearity in isolation transfers energy to short wavelengths, given a spectrum which is peaked at long wavelength or flat, and therefore drives robust production of enstrophy ${ }^{2}$. This term is present even in spectral ranges where the polarization drift nonlinearity dominates $\left(k>k_{0}\right)$. Consequently, enstrophy is not conserved even when the $\mathrm{E} \times \mathrm{B}$ nonlinearity is weak compared to the polarization drift nonlinearity. However, in such a case, the $E \times B$ nonlinearity accounts for proportionately less of the total energy transfer. Because enstrophy production is tied to energy transfer by the EXB nonlinearity, it can be expected that the importance of enstrophy production in the cascade dynamics diminishes for $k>k_{0}$. This fact is also apparent in comparing the enstrophy production rate
$\gamma_{\Omega}=\left[\frac{1}{2} \int|\nabla \tilde{\mathbf{n}}|^{2} \mathrm{~d}^{2} \mathbf{x}\right]^{-1} \mathrm{~L}_{\mathbf{n}} \mathrm{D} \int \nabla^{2} \tilde{\mathbf{n}} \nabla \frac{\partial \tilde{\mathbf{n}}}{\partial \mathbf{y}} \times \mathbf{z} \cdot \nabla \tilde{\mathbf{n}} \mathrm{d}^{2} \mathbf{x}$.

with the nonlinear interaction rate or eddy turnover rate. For $k<k_{0}$, the eddy turnover rate is controlled by the dominant $\mathrm{E} \times \mathrm{B}$ nonlinearity and can be expected to be comparable to the enstrophy generation rate. For $k>k_{0}$ the eddy turnover rate is controlled by the larger polarization drift nonlinearity, while the enstrophy generation rate is tied to the weaker $E \times B$ nonlinearity. Consequently, there will be little enstrophy generation on the nonlinear interaction or nonlinear transfer timescale. This fact suggests a convenient diagnostic of the strength of the polarization drift nonlinearity in the form of the enstrophy dissipation rate $\hat{\gamma}_{\Omega}$ normalized to the mean oscillation time of an intermediate scale mode. Results from the simulations will show $\hat{\gamma}_{\Omega}{ }^{-1}$ to range from $2-3$ in the EXB subrange to hundreds in the polarization subrange.

Energy transfer that is anisotropic and nomlocal in wavenumber space is a robust feature of the EXB nonlinearity, but at variance with the conventional picture of the cascade process. On the other hand, the polarization drift nonlinearity is of the same form as the advective nonlinearity of the vorticity evolution equation of Navier-Stokes turbulence. Therefore, it is reasonable to postulate that the polarization drift nonlinearity produces transfer which is local and isotropic. It is possible to associate these features with symmetries in the structure of the nonlinear coupling. These symmetries are most transparent in the Fourier representation of the nonlinearities. Transforming Eq. (4.1), the evolution of mode amplitudes is given by


where 
$N_{\mathbf{k}}(E \times B)=\frac{i}{2} L_{\mathbf{n}} D \sum_{\mathbf{k}^{\prime}} \mathbf{k} \times \mathbf{k}^{\prime} \cdot \mathbf{z}\left[\mathbf{k}_{\mathbf{y}^{\prime}}-\left(\mathbf{k}_{\mathbf{y}}-\mathbf{k}_{\mathbf{y}^{\prime}}\right)\right] \tilde{\mathbf{n}}_{\mathbf{k}^{\prime}} \tilde{\mathbf{n}}_{\mathbf{k}-\mathbf{k}^{\prime}} \equiv \sum_{\mathbf{k}^{\prime}} \chi_{\mathbf{k}, \mathbf{k}^{\prime}}^{(E \times B)} \tilde{\mathbf{n}}_{\mathbf{k}^{\prime}} \tilde{\mathbf{n}}_{\mathbf{k}-\mathbf{k}^{\prime},}$

$N_{k^{\prime}}(P o l)=\frac{1}{2} \rho_{s}^{3} C_{s} \sum_{\mathbf{k}^{\prime}} \mathbf{k} \times \mathbf{k}^{\prime}-\mathbf{z}\left[\left(\mathbf{k}_{\perp}-\mathbf{k}_{\perp}\right)^{2}-\mathbf{k}_{\perp}{ }^{\prime 2}\right] \tilde{\mathbf{n}}_{\mathbf{k}^{\prime}} \tilde{\mathbf{n}}_{\mathbf{k}-\mathbf{k}^{\prime}}$

$\equiv \sum_{\mathbf{k}^{\prime}} \chi_{\mathbf{k}, \mathbf{k}^{\prime}}^{(\text {Pol) }} \tilde{\mathbf{n}}_{\mathbf{k}^{\prime}} \tilde{\mathbf{n}}_{\mathbf{k}-\mathbf{k}^{\prime}}$

From Eqs. (4.6) and (4.7), the lack of anisotropy in the EXB nonlinearity is evident in the appearance of the factor $\mathbf{k}_{\mathbf{y}}{ }^{\prime}-\left(\mathbf{k}_{\mathbf{y}}-\mathbf{k}_{\mathbf{y}}{ }^{\prime}\right)$, whereas the polarization drift nonlinearity is manifestly isotropic.

Differences between the two nonlinearities regarding the locality of transfer in wavenumber space can also be deduced from Eqs. (4.6) and (4.7). From Eq. (4.6), the ExB coupling is proportional to $\mathbf{k}_{\mathbf{y}}{ }^{\prime}-\left(\mathbf{k}_{\mathbf{y}}-\mathbf{k}_{\mathbf{y}}{ }^{\prime}\right)=2 \mathbf{k}_{\mathbf{y}} \mathbf{y}^{-}-\mathbf{k}_{\mathbf{y}}$. For a nonlocal triad consisting of a long wavelength fluctuation $k_{y}$ interacting with short wavelength fluctuations $k_{y}$ ' and $k_{y}-k_{y^{\prime}}\left(k_{y} \ll k_{y}^{\prime}, k_{y}-k_{y}\right)$, this factor is proportional to the large wavenumber $2 k_{y^{\prime}}$. For local triads $\left(k_{y} \sim k_{y}{ }^{\prime}-k_{y}-k_{y}\right), 2 k_{y}^{\prime}-k_{y} \sim k_{y}$, i.e., the factor is proportional to the small wavenumber. Clearly, the ExB coupling favors nonlocal interaction. Note that this predilection for nonlocal coupling is not isotropic, but applies solely to the displacement in the $\mathrm{k}_{\mathrm{y}}$ direction. By contrast, the comparable coupling factor in the polarization drift nonlinearity is $\left(k_{\perp}-k_{\perp}\right)^{2}-k_{\perp}{ }^{2} \sim k_{\perp}{ }^{2}$ (spectrum symmetries eliminate the contribution $-2 k_{\perp} k_{\perp}$ ). Because this factor involves the squares of the wavenumbers $k_{\perp}{ }^{\prime}$ and $k_{\perp}-k_{\perp}$, the large wavenumber $k_{\perp}{ }^{2}$ cancels and the factor is proportional to the small wavenumber $\mathbf{k}_{\perp}$ 2. Hence, the polarization drift nonlinearity, unlike the $\mathrm{E} \times \mathrm{B}$ nonlinearity, has no special weighting that favors nonlocal coupling (beyond the universal factors $k \times k^{\prime} \cdot z$ and the spectral energy distribution $\tilde{\mathbf{n}}_{\mathbf{k}^{\prime}} \tilde{\mathbf{n}}_{\mathbf{k}-\mathbf{k}^{\prime}}$ ). The symmetries in the nonlinear couplings responsible for this distinction between the $\mathrm{E} \times \mathrm{B}$ and polarization drift nonlinearities derive from the nonadiabatic electron response of the ExB nonlinearity $\left(\phi \sim \delta \partial \bar{n}_{e} / \partial y\right)$ and the adiabatic electron response of the polarization drift nonlinearity $(\phi \sim \tilde{\mathbf{n}})$. These symmetries carry directly over to the energy transfer rates and give rise to a nonlocal transfer rate by the E $\times B$ nonlinearity which dramatically exceeds the local transfer rate, while for the polarization drift nonlinearity, local and nonlocal transfer rates tend to be comparable.

The existence of the frequency spectrum shift is also apparent from Eqs. (4.6) and (4.7) under iteration of the $\tilde{\mathbf{n}}_{\mathbf{k}-\mathbf{k}^{\prime}}$ factor in each expression. In the iteration procedure, $\tilde{\mathbf{n}}_{\mathbf{k}} \mathbf{k}^{\prime}$ is replaced by the formal solution of Eq. (4.5) (with $k \rightarrow k-k$ ). This solution is obtained by placing $N_{k-k^{\prime}}^{(E \times B)}+N_{k-k^{\prime}}^{(P o l)}$ on the right hand side of Eq. (4.5) and inverting the temporal operator of the left hand side. Under standard statistical closures, the sum in the $N_{k-k}$ terms of Eq. (4.5) is restricted to the directly interacting triplet giving

$\tilde{\mathbf{n}}_{\mathbf{k}-\mathbf{k}^{\prime}}=-2 \Delta \omega_{\mathbf{k}-\mathbf{k}^{\prime-1}}\left[\chi_{\mathbf{k}-\mathbf{k}^{\prime},-\mathbf{k}^{\prime}}^{(\mathrm{Pol})}+\chi_{\mathbf{k}-\mathbf{k}^{\prime},-\mathbf{k}^{\prime}}^{(\mathrm{E} \times \mathbf{B})}\right]{\tilde{\mathbf{n}}-\mathbf{k}^{\prime}}_{\tilde{\mathbf{n}}_{\mathbf{k}}}$,

where $\Delta \omega_{\mathrm{k}}{ }^{-1}$ is the inversion of the first four terms of Eq. (4.5). Substitution of Eq. (4.10) into Eqs. (4.6) and (4.7) gives the coherent nonlinear response from each of the nonlinearities. Combining, $\tilde{\mathbf{n}}_{\mathbf{k}}$ evolves according to

$\frac{\partial \tilde{n}_{k}}{\partial t}-D k_{y}^{2} \tilde{n}_{k}+\frac{i V_{D}}{2} k_{y} \tilde{n}_{k}+v_{e f f, i} \tilde{n}_{k}+\mu k^{4} \tilde{n}_{k}-v_{k}^{n} \tilde{n}_{k}=0$,

where

$v_{\mathbf{k}^{\prime}}^{\mathrm{nl}}=2 \sum_{\mathbf{k}^{\prime}}\left[\chi_{\mathbf{k}, \mathbf{k}^{\prime}}^{(\mathrm{Pol})}+\chi_{\mathbf{k}, \mathbf{k}^{\prime}}^{(\mathrm{E} \times \mathrm{B})}\right]\left[\chi_{\mathbf{k}-\mathbf{k}^{\prime},-\mathbf{k}^{\prime}}^{(\mathrm{Pol})}+\chi_{\mathbf{k}-\mathbf{k}^{\prime},-\mathbf{k}^{\prime}}^{(\mathrm{E} \times \mathrm{B})}\right]\left|\tilde{n}_{-\mathbf{k}^{\prime}}\right|^{2} / \Delta \omega_{\mathbf{k}^{-1}}$ 
Note that $\chi^{(\mathrm{Pol})}$ is $90^{\circ}$ out of phase with $\chi^{(\mathrm{E} \times \mathrm{B})}$. Consequently, if the propagator $\Delta \omega_{\mathrm{k}}{ }^{-1}$ is treated as purely real the diagonal terms $\chi_{\mathbf{k}, \mathbf{k}^{\prime}}^{(\mathrm{E} \times \mathrm{B})} \chi_{\mathbf{k}-\mathbf{k}^{\prime},-\mathbf{k}^{\prime}}^{(\mathrm{E} \times \mathrm{B})}$ and $\chi_{\mathbf{k}, \mathbf{k}^{\prime}}^{(\mathrm{Pol})} \chi_{\mathrm{i} \mathbf{k}-\mathbf{k}^{\prime},-\mathbf{k}^{\prime}}^{(\mathrm{Pol})}$ produce coherent damping while the off-diagonal terms $\chi_{\mathbf{k}, \mathbf{k}^{\prime}}^{(\mathrm{E} \times \mathrm{B})} \chi_{\mathrm{k}-\mathrm{k}^{\prime},-\mathrm{k}^{\prime}}^{(\mathrm{Pol})}$ and $\chi_{\mathbf{k}, \mathrm{k}^{\prime}}^{(\mathrm{Pol})} \chi_{\mathbf{k}-k^{\prime},-k^{\prime}}^{(\mathrm{E} \times \mathrm{B})}$ produce a phase oscillation. Accordingly, the diagonal terms are related to the spectrum linewidth ${ }^{11}$ while the off-diagonal terms contribute to the frequency of the spectrum peak ${ }^{10}$. Physically, the frequency shift arises from the interaction of a driven fluctuation $\tilde{\mathbf{n}}_{k-k^{\prime}}$ which is $90^{\circ}$ out of phase with the test and beat waves at $\mathbf{k}$ and $\mathbf{k}$. Clearly it is cross-coupling of modes driven by each of the two nonlinearities that produces the shift, with no shift occurring if either of the nonlinearities is absent.

Substituting for $\chi^{(\mathrm{ExB})}$ and $\chi^{(\mathrm{Pol})}$ in Eq. (4.10), it is seen that

$\operatorname{Im} v_{\mathbf{k}^{\mathrm{n}}}^{\mathrm{nl}}=-\mathrm{L}_{\mathbf{n}} \mathrm{D} \rho_{\mathrm{s}}{ }^{3} \mathrm{C}_{\mathrm{s}} \sum_{\mathbf{k}^{\prime}}\left(\mathbf{k} \times \mathbf{k}^{\prime} \cdot \mathbf{z}\right)^{2} \mathbf{k}_{\mathbf{y}}\left[2 \mathrm{k}_{\perp}{ }^{2}-\mathrm{k}_{\perp}{ }^{2}\right]\left|\mathrm{n}_{\mathbf{k}} \cdot\right|^{2} / \operatorname{Re}\left(\Delta \omega_{\mathbf{k}^{-1}}\right.$,

corresponding to an upshift for intermediate modes $k_{\perp}{ }^{2} \geq\left(k_{\perp}{ }^{2}\right\rangle$. The qualitative features of this predicted shift, specifically its sign and its $\mathbf{k}_{\mathbf{y}}$ dependence, will be examined in Sec. IV.

\subsection{Spectral Transfer}

In this section the spectral flow of energy over the spectrum range is described. The transfer properties are inferred from the time evolution of the wavenumber spectrum and the time evolution of the energy and enstrophy transfer rates from (or to) selected bands in wavenumber space ${ }^{2}$. The energy and enstrophy transfer rates due to the $E \times B$ nonlinearity are given in Ref. 2. With the addition of the polarization drift nonlinearity, the evolution of the energy and enstrophy of the mode $k$ is described by

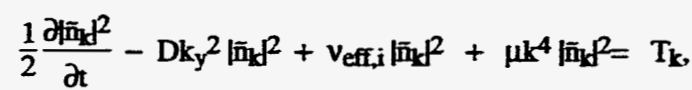

$\frac{1}{2} \frac{\partial k^{2}\left|\tilde{n}_{k}\right|^{2}}{\partial t}-D k_{y}^{2} k^{2}\left|\tilde{n}_{k}\right|^{2}+\left.v_{e f f i} k^{2} \psi_{\tilde{n}_{k}}\right|^{2}+\mu k^{6}\left|\tilde{n}_{k}\right|^{2}=U_{k}$,

where

$\mathrm{T}_{\mathbf{k}}=\mathrm{L}_{\mathbf{n}} \mathrm{D} \operatorname{Im} \sum_{\mathbf{k}}\left(\mathbf{k} \times \mathbf{k}^{\prime} \cdot \mathbf{z}\right) \mathbf{k}_{\mathbf{y}}{ }^{\prime} \tilde{\mathbf{n}}_{\mathbf{k}} \tilde{\mathbf{n}}_{\mathbf{k}-\mathbf{k}} \cdot \tilde{\mathbf{n}}_{\mathbf{k}}{ }^{*}$

$-\rho_{s}^{3} C_{s} \operatorname{Re} \sum_{\mathbf{k}^{\prime}} \mathbf{k} \times \mathbf{k}^{\prime} \cdot \mathbf{z}\left(\mathbf{k}_{\perp}-\mathbf{k}_{\perp}\right)^{2} \tilde{\mathbf{n}}_{\mathbf{k}} \cdot \tilde{\mathbf{n}}_{\mathbf{k}-\mathbf{k}^{\prime} \overline{\mathbf{n}}_{\mathbf{k}}}{ }^{*}$

and

$U_{\mathbf{k}}=L_{\mathbf{n}} D \operatorname{Im} \sum_{\mathbf{k}}\left(\mathbf{k} \times \mathbf{k}^{\prime} \cdot \mathbf{z}\right) \mathbf{k}^{2} \mathbf{k}_{\mathbf{y}} \tilde{\mathbf{n}}_{\mathbf{k}} \cdot \tilde{\mathbf{n}}_{\mathbf{k}-\mathbf{k}} \tilde{\mathbf{n}}_{\mathbf{k}}^{*}$

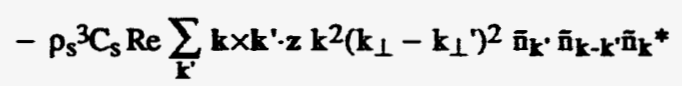

are respectively the energy and enstrophy transfer rates from the mode $k$. Note that summing $U_{k}$ over all $k$ yields the enstrophy production rate, Eq. (4.4). Summing $T_{k}$ and $U_{k}$ over a band of wavenumbers (typically with $k_{y}=$ constant or $k_{x}=$ constant) yields the transfer from the given band. Moreover, by selecting only certain $k$ ' values in the sums, the transfer rates can be formulated to identify transfer between coupled triplets with wavenumbers which span more than a significant fraction of the spectrum (typically $1 / 3$ to 1/2). This allows a quantitative assessment of the relative magnitudes of local and nonlocal transfer. Before describing the flow induced by the $\mathrm{E} \times \mathrm{B}$ and polarization drift nonlinearities together, it is useful to establish a reference in terms of the flow properties under each of the nonlinearities in isolation. 
A. Transfer Properties of Each Nonlinearity in Isolation

Spectral flow by the ExB nonlinearity was described in Ref. 2 and is briefly summarized. In absence of driving and damping, spectra initially peaked at low $\mathrm{k}$ relax to the equipartitioned state predicted by statistical mechanics. The relaxation is accomplished by a prompt nonlocal flow to the largest $k_{y}$ bands (defined by $k_{y}-k_{y} \max$ ), producing, within a few eddy turnover times, spectra with peaks at high $\mathbb{k}_{\mathbf{y}} \mathrm{L}$. Subsequent sloshing motion excites the intermediate wavenumbers. The energy transfer rate between nonlocally displaced $\mathrm{k}_{\mathrm{y}}$ bands exceeds the transfer between closely neighboring (local) bands by an order of magnitude. Local and nonlocal transfer rates in the $\mathbf{k}_{\mathbf{x}}$ direction are comparable. In both directions, transfer proceeds to high $\mathrm{k}$ when the spectrum is initially peaked. When driven by modes at low $\mathbf{k}$ and damped by modes at high $\mathbf{k}$ with an intermediate inertial range, the stationary wavenumber spectrum is remarkably flat over the inertial range, falling off only at the damped modes. This dramatic deviation of the spectrum from that predicted by similarity arguments is another indication of strong nonlocal transfer.

When the polarization drift nonlinearity is the sole nonlinear coupling mechanism. the equilibrium spectrum peaks at low $k$. Figure 4.1 shows the equilibrium spectrum (no driving or damping) reached from an initial spectrum with $\left.\ln _{\mathbf{k}}\right|^{2} \sim \mathrm{k}^{-3}$ for $41 \times 41$ modes.



Fig. 4.1 Equilibrium spectrum of undriven/undamped turbulence with the only polarization drift nonlinearity. This spectrum is the relaxed state that has evolved from an initial spectrum with a fall-off index of $a=-4$. The initial spectrum is identical to the initial spectrum shown in Fig. 1 of Ref. 2 . 
Relative to the initial spectrum, the equilibrium spectrum is more peaked at lower wavenumbers and flatter at large wavenumbers, indicating an inverse cascade of energy to low $k$, but also some energy transfer to high $k$. Indeed, the energy transfer rate reveals such a transfer pattern. From Fig. 4.2, the transfer rate $T_{k}$ is positive at the lowest wavenumbers and negative somewhat higher, indicating a primary transfer into the lowest wavenumbers from the higher band. The higher band also engages in a smaller subsidiary transfer to even higher wavenumbers, as seen in the positive peak just above the negative feature. In the upper half of the spectrum there is little net transfer above the noise level as defined by the standard deviation of the scatter in the transfer history.

The transfer to low $\mathbf{k}$ is consistent with the dynamical constraints imposed by the conservation of energy and enstrophy. The smaller forward cascade might appear to be a violation of these constraints. However, as shown elsewhere ${ }^{12}$, some energy must be transferred in the forward direction in order to generate sufficient enstrophy to compensate for the enstrophy destroyed by the inverse energy cascade. The amount of energy transferred to high $\mathrm{k}$ decreases to zero as the largest wavenumber goes to infinity. For the rather small wavenumber spaces considered here, however, this energy can be appreciable This process contributes to the existence of two fall-off rates in the equilibrium spectrum, a feature not anticipated by equilibrium statistical mechanics.

As discussed in the previous section, the symmetries of the coupling coefficient of the polarization drift nonlinearity suggest that nonlocal transfer should be no larger than the local transfer. In fact, as evidenced by Fig. 4.3, the local transfer rate from the band with negative transfer in Fig. 4.2 exceeds ihe nonlocal transfer rate by a factor of two or three. Moreover, the transfer rates in the $\mathrm{k}_{\mathrm{x}}$ and $\mathrm{k}_{\mathrm{y}}$ directions are found to be comparable to within a factor of two, so that transfer is isotropic, as anticipated from the form of the nonlinearity.



Fig- 4.2 Time averaged energy transfer rate during the relacation leading to the spectrum of Fig 4.1. The error bers inficate the standard deviation from the mean value ploted in the figure.



Fig. 4.3 Local and nonlocal energy transfer ranes from the band with negative transfer (energy ouflow) during the retacation leading to the spectrom of Fig 4.1 


\section{B. Transfer Properties with Both Nonlinearities}

With both nonlinearities present, the transfer of exergy and enstrophy is characterized by three general principles. These are now presented. 1) Excepting the effects represented by the other two principles, each nonlinearity transfers energy and enstrophy throughout the spectrum as it would if acting alone. Because the EXB nonlinearity dominates at low $\mathbf{k}\left(\mathbf{k}<\mathrm{k}_{0}\right)$, the net transfer in the long wavelength subrange is similar to that produced by the $\mathrm{E} \times \mathrm{B}$ nonlinearity alone, ie., the transfer is to high $\mathrm{k}$ with a strong nonlocal component in $k_{y}$. While the EXB dynamics are similar in the high $\mathbf{k}$ subrange, the transfer they produce is small by cumparison with that of the polarization drift nonlinearity, which drives a dual cascade via local, isotropic transfer.

2) With the two nonlinearities acting together, the spectral distribution of energy tinf is modified relative to its configuration with a single nonlinearity. Since $T_{k}$ and $U_{k}$ depend on amplitudes $\tilde{\mathbf{n}}_{\mathfrak{k}}, \overline{\mathbf{m}}_{\mathbf{k}} \mathfrak{k}^{\prime}$, and $\overline{\mathbf{n}}_{\mathbf{k}}{ }^{*}$ [Eqs. (14) and (15)], the transfer is also modified relative to its behavior for a single nonlinearity. In each of the subranges, the spectra. both the equilibrium and the driven/damped stationary spectrum, tend to book like the spectra of the dominant nonlinearity, when acting alone. Consequently, transfer well within each subrange continues to be characterized by the transfer of the dominant nonlinearity in isolation. Significant modifications are thus restricted to the crossover region around $k_{0}$ where the two nonlinearities are comparable.

3) The spectrum shift, Eq. (4.10), affects the transfer in three ways. a) The spectrum shift contributes to the three wave phase decorrelation just as a linear frequency mismatch contributes to the decorrelation. The shift mismatch $v_{\mathbf{k}}+v_{\mathbf{k}} \mathbf{k}^{\prime}-v_{\mathbf{k}}$ is finite, due to the dispersion of the shift, and more pronounced for nonlocal triads than local triads. The shift-induced phase decomelation will therefore limil nonlocal transfer. However. this effect is a factor only in the crossover region. Outside this region. the shift-induced decorrelation is small relative to the phase decorrelation caused by the eddy damping of the dominant nonlinearity. b) In a strong tubulence regime, the frequency shift in the response function of the driven fluctuation gives rise to a nonresonant contribution [off-diagonal terms in Eq (4.10)] to the eddy damping which can, in effect, be comparable to the resonam (diagonal) contribution. In such a case, the transfer, or equivalently, the energetics, is directly affected by the shif. c) The frequency shift affects the nonlinear stability (or free energy extraction) by modifying Re $\omega$ in the eigenmode potential. This effect is most simply displayed in typical drift wave growth rates, which contain the factor $\omega$ - $\omega_{\text {n. }}$, with instability for $\omega<\omega_{\text {. }}$ The role of the frequency shift on noalinear stability, as it pertains to dissipative drift waves, is explored in Ref. (10). As noted previously, this effect is not presemt in the present computational model but will be examined computationally in the next chapter.

It should be apparent from the above that the transfer dywamics of the crossover region is the most difficult to analyze. Figures (4.4) and (4.5) depict the evolution of the spectrum in the crossover region under non driven, non damped relaxation, with $k_{0}$ shightly larger than the median $k$. Figure (4.4) is the spectrum a few eddy turnover times afier commencing the retaxation from a initial $\mathrm{k}^{3}$ distribution. Significant nonlocal transfer to high $\mathbf{k}$ modes is evident in the peaks which have developed at high llt. This peaking feature is isotropic in $k$ and contrasts with the peaks that occur at high $k_{y}$ (but not $k_{x}$ ) ai comparable times with the ExB nonlinearity alone. The nonlocal transfer is an unmistakable signatme of the EXB nonlinearity. whereas the tendency toward isotropy is a feature of the polarization drift nonlinearity. Isotropization of the high $\mathbf{k}$ peaks is accomplished by transfer modifications induced by the spectrum distribution (principle 2). The final equilibrium spectrum. Fig (4.5), is mostly flat with a narrow peak at low $k$. The peak is produced by the inverse cascade of the polarization drift nonlinearity. It is more narrow 

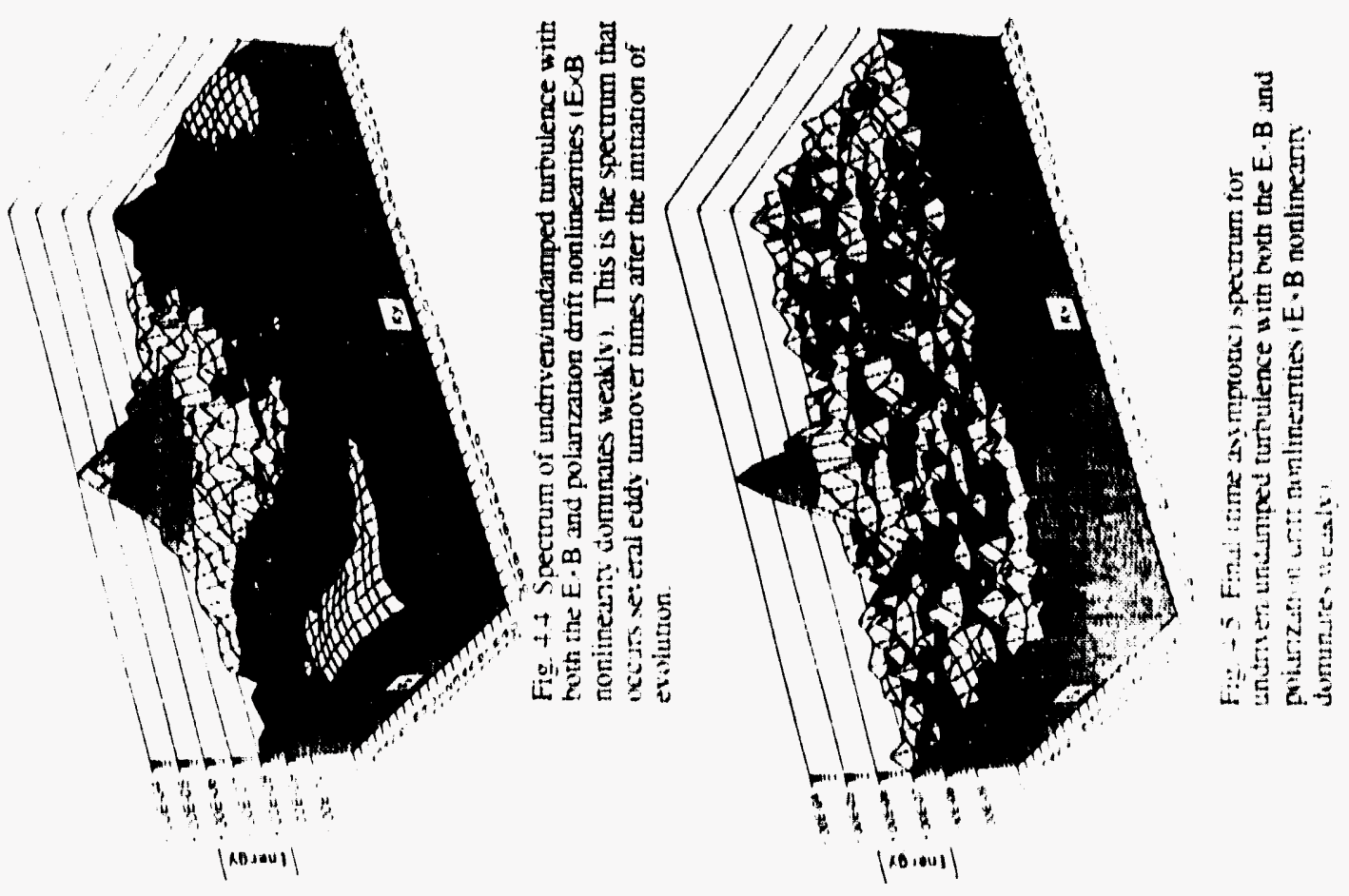




The flat portion is atributable to the EXB nonlinearity, which vends to drive the spectrum to equipartition. In this regard, it is interesting to note that the spectrum predicted by equilibrium statistical mechanics is the equipartitioned spectrum. since enstrophy is not conserved in this case. Statistical mecthanics is umable to account for the inverse cascade becanse the EXB nonlinearity breaks enstrophy invariance even though significant enstrophy conserving transfer by the polarization diff nontinearity occurs.

The degree of nonconservation of enserophy, or more precisely. the rate of enstrophy generation, is a measure of the extem of energy transfer to high $k$ by the EXB nonlinearity. In Fig. (4.6), the enstrophy evolution ander non driven, ron damped relaxation is plotted as a function of time for four spectrum configurations. These are the EXB and polarization subranges, in which $k_{0}$ falls at either the exweme low end (polarization sabrange) or high end of the spectum (EXB subrange); and two crossover region configurations, one in which $k_{0}$ is slightly so the right of center and one in which $k_{0}$ falls slightity to the left of center. In the EXB subrange there is significant enstrophy generation. and the timescale of enstrophy production is a few edty turnover times. These fects indicate that the transfer dynamics is dominated by the forward cascade of the ExB nonlinearity. In contrast, the small net enstrophy increase and long (many eddy turnover times) generation timescale evident in the polarization subrange, indicate that the forward transfer by the EXB nonlinearity is of minor overall importance In the crossover region. the same general trend is evidem, with the net enstrophy production and production rate being ronghly proportional to the fraction of $k$ space below $k_{0}$. Taken together, these resnits suggest that in a spectrum encompassing both subranges, there is significant non-enstrophy conserving forward transfer from $k_{\min }$ to $k_{0}$ and somewhat beyond, but that its magnitude relative to the net transfer rapidly diminishes well beyond $k_{0}$

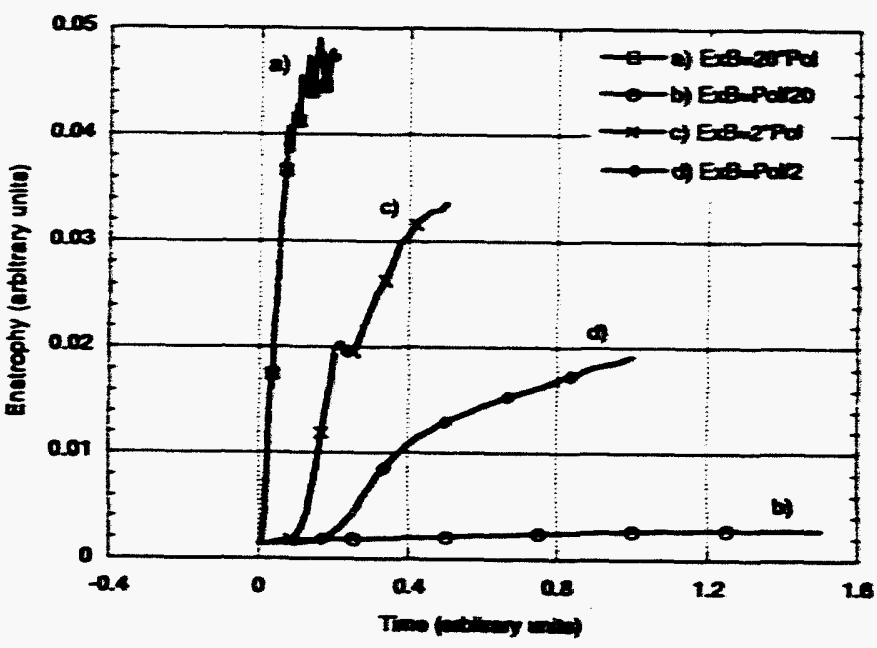

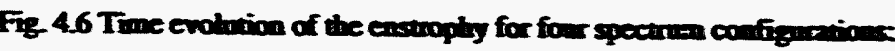

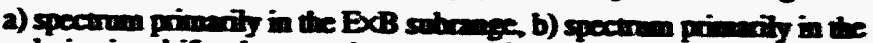

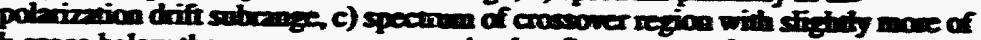

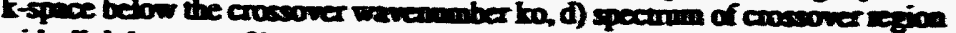

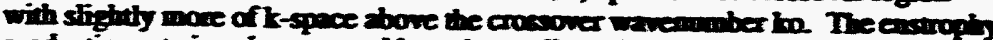

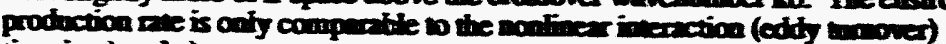
ime in a) and c). 
ii is obviously imponant to examine the transfer dynamics in a saturated spectrum achieved through the dynamic balance of sources, sinks and transfer. For dissipative trapped ion convective cells. turbulence is driven ai long wavelength (below $k$, t: at longer wavelengths still. ion-ion collisions provide a sink. The rohust forward transfer of the E.B nonlineariy can be expected to carn encrgy hevond $k$. A: some seale the energy is

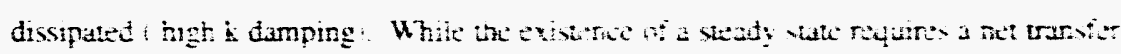

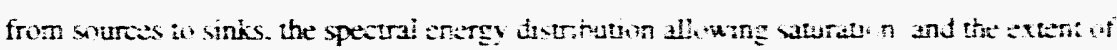

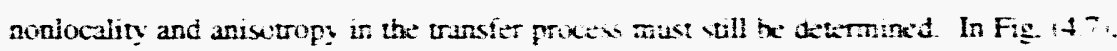
the staionary spectrum is plotted for a cenfiguration in which $k_{\text {, }}$ is centrally iocated hetuten $k_{\min }$ and $k_{\max }$. Unstable modes occur in the low $\mathbf{k}$ region with a stable band at the lowest wavenumbers, representing ion-ion collisional damping. A broad inerial range extends to the highest wavenumbers where a byperviscous damping provides $\mathbf{a}$ high $\mathbf{k} \sin \mathbf{k}$. Figure (4.7) reveals a spectrum with sharp peaks at the wavenumbers of the driven modes. surrounded by a plateau-like region of roughly elliptical shape. Beyond the platean. the spectrum falls off sharply to the edge. The edge of the plateau corresponds roughly to the crossover point $k_{0}$. Comparison with the stationary spectrum of the $E \times B$ nonlinearity alone, which is flat all the way out to the viscously damped modes ${ }^{2}$, indicates that significant nonlocal transfer to high $\mathbf{k}$ is the dominant process in the platean region. Anisotropic transfer is the cause of the elliptical stape of the platean. There is also transfer to the collisionally damped modes at low $k$ producing a steep drop-off in going below the driven wavenumbers. This spectrum indicates that the characteristics of the $E \times B$ and polarization drift nonlinearities effectively dominate in the appropriate ranges of the spectrum.

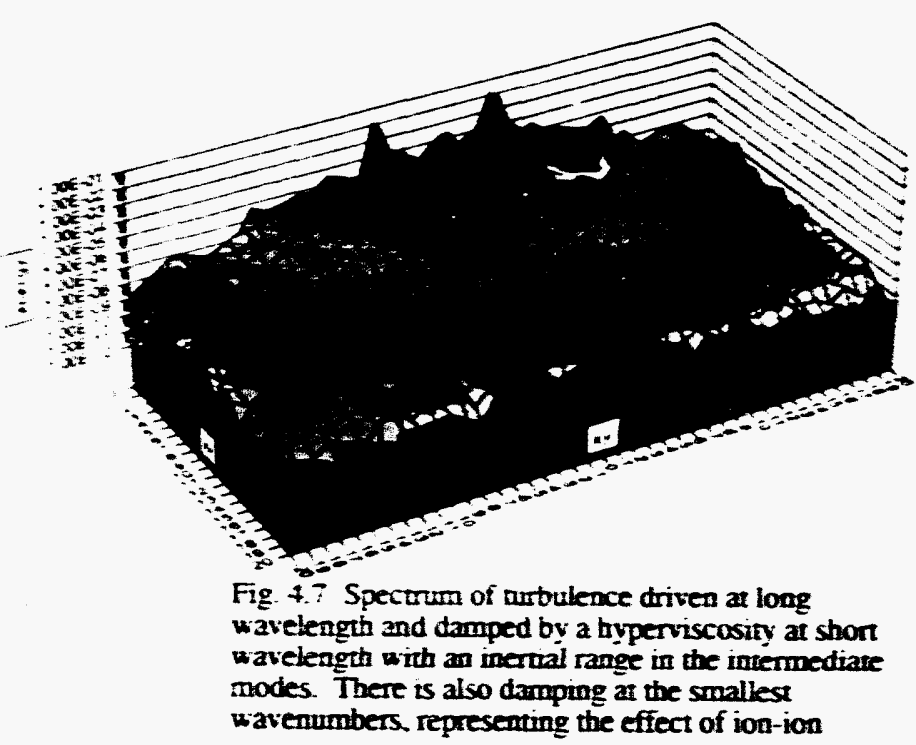



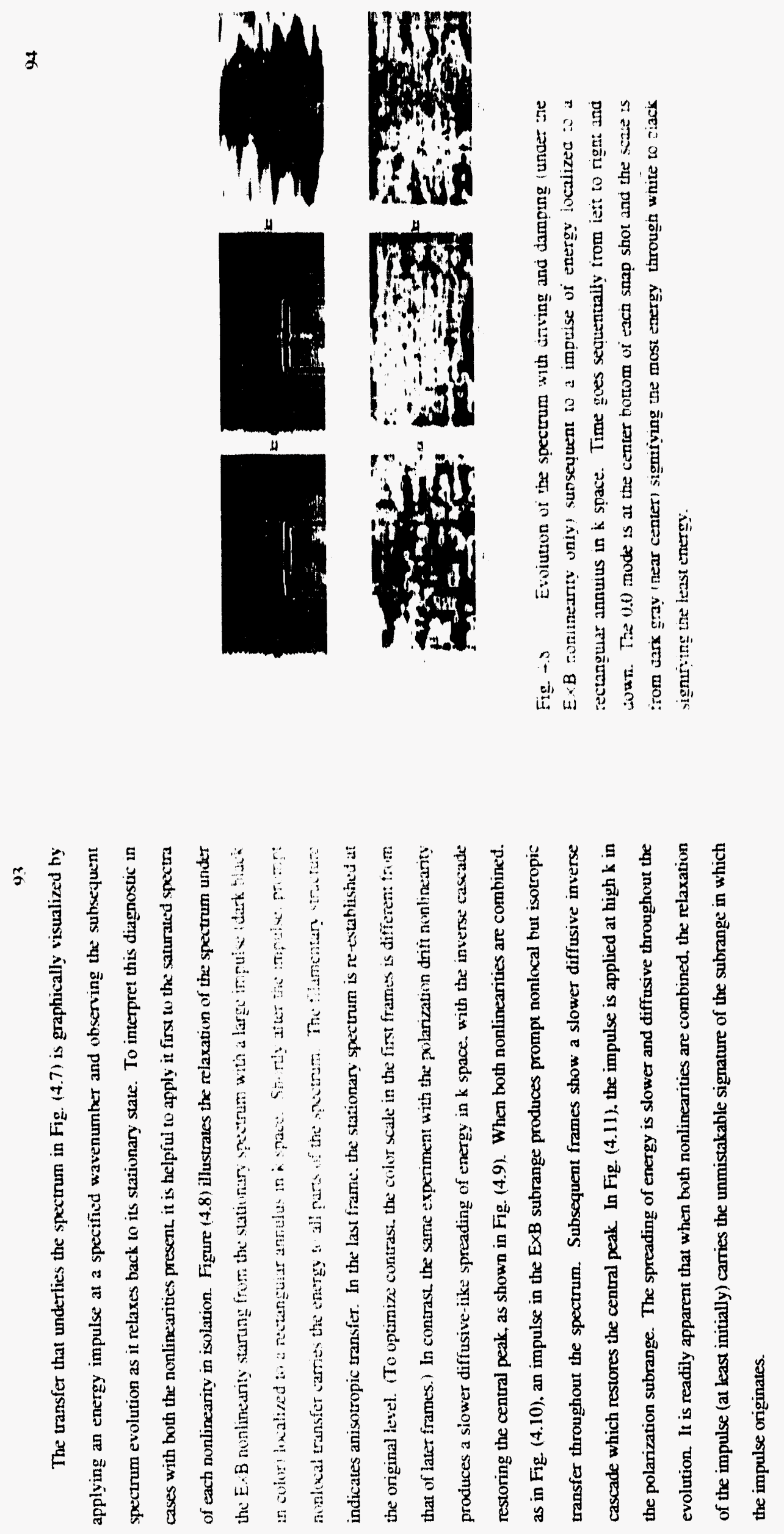

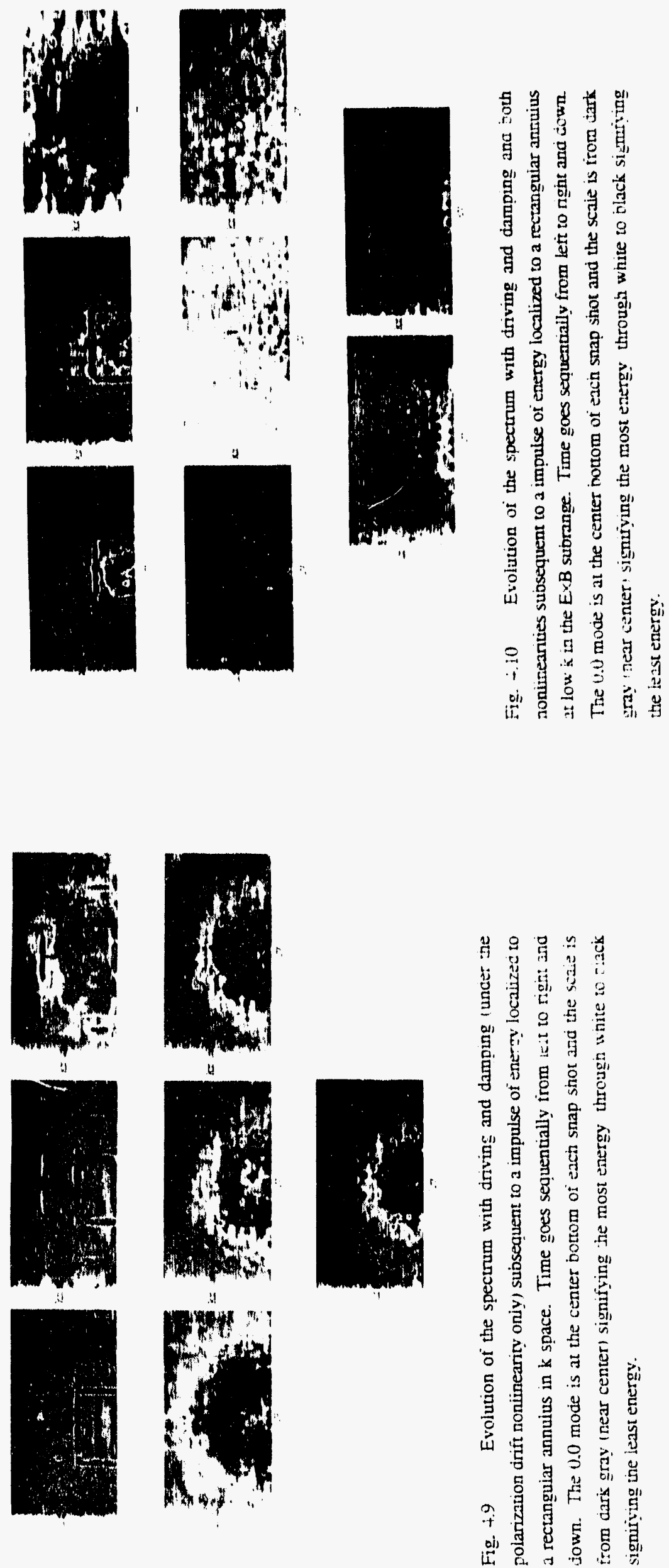

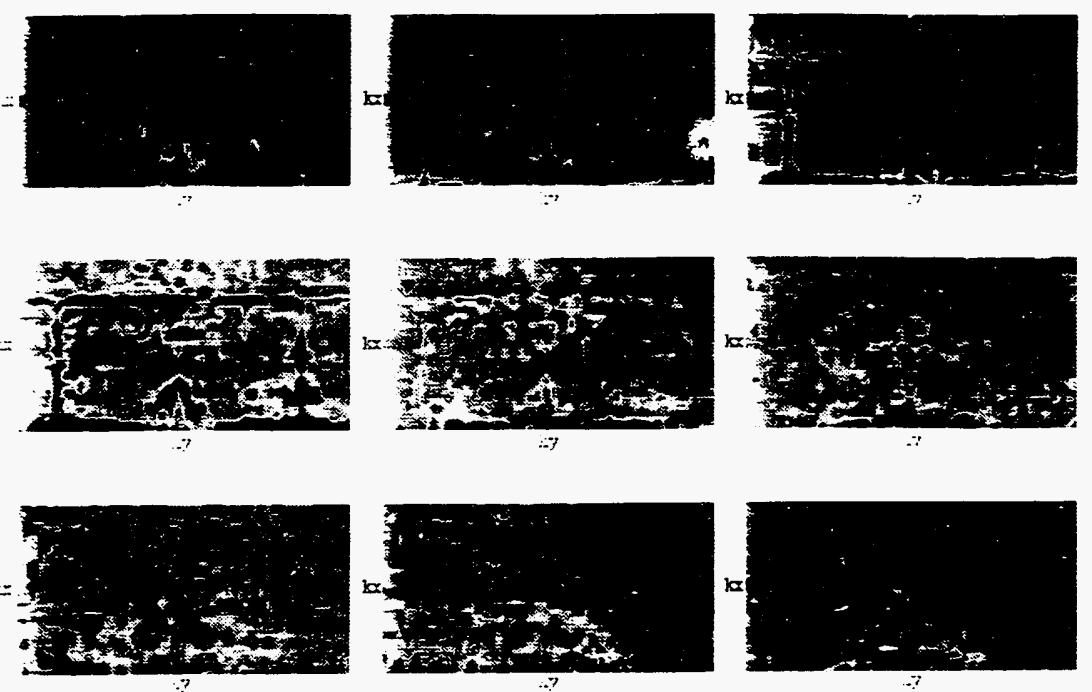

Fig. t.11 Evolution of the spectrum with driving and damping and both noniinearicies subsequent to a impulse of energy locaiized to a rectangular annuius at high $\mathbf{k}$ in the polarization drift subrange. Time goes sequentially from ieft to right and down. The 0.0 mode is at the center bonom of each snap shot and the scale is from dark gray (near center) signifying the most energy through winite to black signifying the least energy.

\subsection{Frequency Spectrum}

The frequency spectrum is generally taken to signify the frequency dependent part of the power spectrum $S_{k}(\omega)$, for $k$ fixed. In terms of the solution of a spectral representation of the model, i.e., the solution of Eq. (4.5), the frequency spectrum is just the Fourier transform of the temporal autocorrelation function, obtained from the time history of the mode $\mathbf{k}$,

$S_{k}(\omega)=\frac{1}{\sqrt{2} \pi} \int d \tau \exp (-i \omega \tau)\left\langle\tilde{n}_{\mathbf{k}}\left(t^{\prime}\right) \tilde{n}_{\mathbf{k}}^{*}\left(t^{\prime}+\tau\right)\right\rangle$,

where \langle\rangle is the average over a suitably chosen ensemble.

In this section, frequency spectra are examined for a variety of modes and conditions with the objective of establishing qualitatively the existence of a frequency shift induced by the coupling of the ExB and polarization drift nonlinearities. From Eq. (4.9), it is apparent that the spectrum will contain a shift, given by Eq. (4.11). However, it should also be noted that the spectrum is a correlation, and the correlation of $\tilde{n}_{k}\left(t^{\prime}\right)$ with $\tilde{n}_{k}{ }^{*}\left(t^{\prime}+\tau\right)$ for small time differences $\tau$ is not captured by simply substituting into Eq. (4.16) the solution of Eq. (4.9), an approximate one-time, one-point equation ${ }^{13}$. Specifically, Eq. (4.9) represents the coherent response, but does not capture the incoherent emission associated with small scale, short time correlation. While the dissipative part of the incoherent emission ultimately balances the coherent decay in a steady state inertial range, and thus provides a link between the two components (and a way of determining the linewidth ${ }^{14}$ ), the reactive component of the incoherent emission is not related to the reactive part of the coherent response, $\operatorname{Im} \mathbf{v}_{\mathbf{k}}$, in any simple way. Thus, it is simplistic to expect that the frequency spectrum, and the frequency shift in particular, are totally described by the solution of Eq. (4.11) ${ }^{15}$. Indeed, as will be apparent, the frequency spectra are complicated and not readily interpreted as a 
localized, single peaked function. Nevertheless it is apparent that a large shift in the mean frequency of the spectrum occurs, that the shift requires the presence of both nonlinearities, and that other features of Eq. (4.11) are present.

The frequency spectra of both saturated (driven/damped) turbulence and relaxing (no driving or damping) turbulence have been determined. In general, the spectra of saturated turbulence are much broader than the spectra of relaxing turbulence, and the shifts relative to the linewidths are accordingly less pronounced. The spectra of relaxing turbulence, on the other hand, are not stationary. Computing the spectra from a time history which covers the entire relaxation phase, these spectra have a feature associated with the relaxation. Since the relaxation takes many eddy tumover times, and the timescale of the shift is typically less than an eddy turnover time, the nonstationarity of the spectrum poses no particular difficulty in examining the shifts. At most, the nonstationarity is responsible for intrinsic broadening of the spectrum, but by an amount that is small relative to the magnitude of the shifts. For the above reasons, the spectra shown in the figures are for relaxing turbulence.

Figure (4.12) shows the frequency spectra of a mode with small wavenumber for relaxing turbulence with the $\mathrm{X} \times \mathrm{B}$ nonlinearity only, the polarization drift nonlinearity only, and both nonlinearities. In the latter case there is a marked shift of the spectrum peak to higher frequency. In all cases, the peaks are in the electron diamagnetic direction. In Fig. (4.13), the spectra for a mode with large wavenumber are displayed. For the cases with $\mathrm{E} \times \mathrm{B}$ and polarization drift nonlinearities only, the spectra are peaked at the small frequency corresponding to the (linear) diamagnetic rotation [third term, Eq. (4.5)]. With the two nonlinearities combined, the spectrum becomes highly complicated, acquiring a small reproducible feature in the ion direction, and a much larger feature at high frequency in the electron direction. The mean frequency $\bar{\omega}_{\mathbf{k}}=\int d \omega \omega S_{\mathbf{k}}(\omega)$ is dramatically raised, as is the width, $\Delta \omega_{\mathrm{k}}^{2}=\int \mathrm{d} \omega\left(\omega^{2}-\bar{\omega}_{\mathrm{k}}{ }^{2}\right) \mathrm{S}_{\mathrm{k}}(\omega)$, relative to the cases with a single nonlinearity. For the combined case of Fig. (4.13), $\bar{\omega}_{\mathrm{k}} \cong 2 \Delta \omega_{\mathrm{k}}$. From these figures, it is clear that there is a marked frequency shift associated with the coexistence of the two nonlinearities. The magnitude of the shift, in absolute terms, is larger for the mode with larger wavenumber. For the shifted spectra of Figs. (4.12) and (4.13), $\bar{\omega}_{\mathbf{K}}$ large $\sqrt{\omega_{k} \text { small }} \approx 25$, a number of the same magnitude as the ratio of the wavenumbers. Both of these features are consistent with the shift described by Eq. (4.13). The same general features are also found in the spectra of steady state (driven/damped) turbulence computed numerically for the same model. However, due to the very broad linewidths of the steady state case, these features are less pronounced.

Given the complexity of the frequency spectrum, especially for high $k$, a more quantitative comparison with theory is beyond the scope of the present work. The next chapter will focus on a theoretical understanding of the complex features and inclusion of the feedback of spectrum shifts on the mode stability in the simulations. The existence of a large frequency shift at the longer wavelengths required for the $\mathrm{E} \times \mathrm{B}$ nonlinearity is interesting in light of the apparent observation of long wavelength fluctuations with large phase velocity $\left(v_{\mathrm{ph}}>\omega_{*} / \mathbb{k}\right)$ in the Texas Experimental Tokamak $\left(\right.$ TEXT) ${ }^{16}$. 


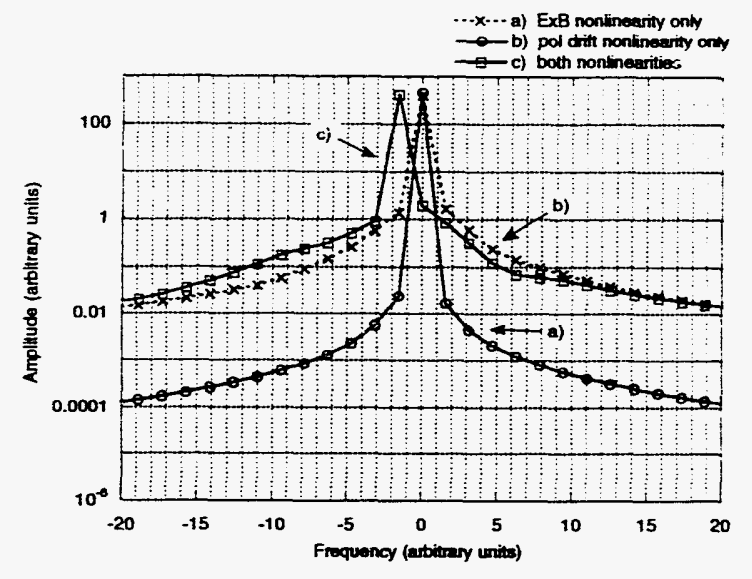

Fig. 4.12 Frequency spectra of a mode with small wavenumber during relaxation without driving or damping. The spectra correspond to a) ExB nonlinearity only, b) polarization drift nonlinearity only, c) both nonlinearities

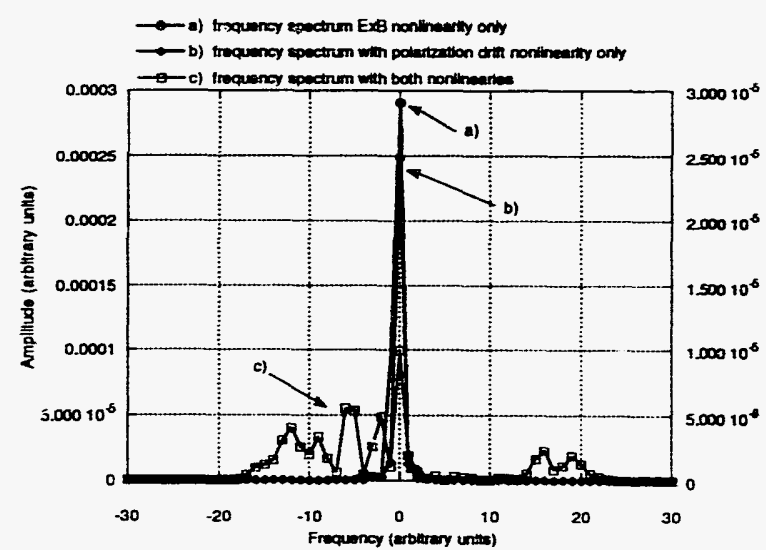

Fig. 4.13 Frequency spectra of a mode with large wavenumber during relaxation without driving or damping. The spectra correspond to a) EXB nonlinearity only b) polarization drift nonlinearity only, c) rsui nonlinearities

\subsection{Conclusions}

The spectral transfer dynamics of drift wave turbulence over the broad wavenumber range incorporating both the $\mathrm{E} \times \mathrm{B}$ and polarization drift nonlinearities has been explored. This work is based on numerical solution of the single field dissipative trapped ion convective cell turbulence model 1,2 and describes the spectral transfer resulting from unstable trapped ion modes at long wavelength. The present computational model is based on an "i $\delta$ " approximation with laminar electron dynamics resulting from collisional, nonadiabatic electrons. Consequently, the present computational model neglects the dynamical feedback of finite amplitude nonlinear-induced frequencies on mode stability.

A variety of diagnostics, including wavenumber and frequency spectra, spectrum histories, energy and enstrophy transfer rates, and spectrum impulses, are employed to characterize the transfer. It is found that the $\mathrm{E} \times \mathrm{B}$ and polarization drift nonlinearities transfer energy (and enstrophy) much as they would in isolation. Consequently, there are two subranges corresponding to spectral regions in which one nonlinearity or the other is dominant. In the low $\mathrm{k}$ EXB subrange, the net energy transfer is directed to higher $\mathrm{k}$ with a very large nonlocal component in the $\mathrm{k}_{\mathrm{y}}$ direction. There is significant generation of enstrophy on the timescale of a few eddy turnover times. In the high $\mathbf{k}$ polarization drift subrange, transfer is local in $\mathbf{k}$. There is evidence for a dual cascade, and enstrophy generation is weak, requiring many eddy turnover times for any change in magnitude. The near conservation of enstrophy on dynamical timescales is consistent with the observed inverse cascade of energy. For saturated turbulence which is driven at low $\mathbf{k}$, damped at lower $\mathrm{k}$ and at high $\mathrm{k}$, with an inertial range providing a bridge between the driven wavenumbers and the short wavelength damping, the spectrum is flat in the EXB subrange and falls off in the polarization drift subrange. Nonlocal transfer is responsible for the flatness of the spectrum at low $\mathrm{k}$ 
In the crossover region where the two nonlinearities are comparable there is a rich cross-coupling. Energy is transferred nonlocally but the transfer tends to be isotropic. A significant shift in the peak of the frequency spectrum occurs. This shift is directly atributable to the coupling of the two nonlinearities, as it disappears if either nonlinearity is set to zero. The shift increases with increasing wavenumber. Both of these effects are consistent with the theoretical shift based on the cross-coupling of the two nonlinearities in the renormalized response function. In the present work, model limitations have prevented treatment of the back-reaction of the shift on the mode dynamics.

The visualization of energy flow by following the spectral relaxation after application of a localized impulse energy at a given wavenumber is found to provide a reliable and graphic indication of energy flow, indicating accurately its direction, its locaiity in wavenumber space and whether transfer is isotropic.

In the next chapter, work on this model will include the frequency shift feedback and its modification of the mode stability structure. In addition, an investigation will be made of structures which are stable solutions of one nonlinearity and their persistence in the presence of both nonlinearities.

\section{References}

1. P.H. Diamond and H. Biglari, Phys. Rev. Lett 65, 2865 (1990)

2. D.E. Newman, P.W. Terry, and P.H. Diamond, Phys. Fluids B 4, 599 (1992).

3. R.J. Fonck, S.F. Paul, D.R. Roberts, Y.J. Kim, N. Bretz, D. Johnson, R Nazikian, and G. Taylor, 18th European Conference of Controlled Fusion and Plasma Physics, Berlin, Germany, edited by P. Bachmann and D.C. Robinson (European Physical Society, Geneva, Vol. 15C, Part I, 1991) p. I-269.

4. P. Cripwell and A.E. Costley, 18th European Conference of Controlled Fusion and Plasma Physics, Berlin, Germany, edited by P. Bachmann and D.C. Robinson (European Physical Society, Geneva, Vol. 15C, Part I, 1991) p. I-17.

5. P.M. Schoch, J.C. Forster, W.C. Jennings, and R.L. Hickok, Rev. Sci. Instrum. 59, 1825 (1986)

6. D.L. Brower, W.A. Peebles, and N.C. Luhmann, Nucl. Fusion 27, 2055 (1987).

P.W. Terry and W. Horton, Phys. Fluids 25, 491 (1982).

A. Hasegawa and K. Mima Phys. Rev. Lett 39, 205 (1977)

A. Hasegawa and K. Mima, Phys. Rev. Lett. 39, 205 (197

10. Y.-M Liang, P.H. Diamond, X.H. Wang, D.E. Newman, and P.W. Terry, "A Two-Nonlinearity Model of Dissipative Drift Wave Turbulence", Phys. Fluids B, Two-Nonltited

11. P.W. Terry and P.H. Diamond, in Statistical Physics and Chaos in Fusion Plasmas ed. by W. Horton and L. Reichl (Wiley, N.Y., 1984) p. 335; and P.W. Terry and P.H. Diamond, Phys. Fluids 28, 1419 (1985).

12. P.W. Terry and D.E. Newman, Bull. Amer. Phys. Soc. 37 (1992).

13. T. Boutros-Ghali and T.H. Dupree, Phys. Fluids 24, 1839 (1981).

14. The line width is not directly given by the coherent damping. In steady state coherent damping is balanced by driving and incoherent emission and all of these processes affect the spectrum. This is discussed in the articles of Ref. 11.

15. This is further discussed in N. Mattor and P.W. Terry, Phys. Fluids B 4, 1126 (1992).

16. D.W. Ross, R.V. Bravenec, Ch. P. Ritz, M. L. Sloan, J.R. Thompson, A.J. Wootton, PM Schoch, J.W. Heard, T.P. Crowley, RI Hickok V Simcic, D.L. Brower, W.A. Peebles, and N.C. Luhmann, Jr., Phys. Fluids B 3, 2251 (1991). 


\section{Chapter 5 \\ Multiple Field effects}

\subsection{Introduction}

One of the more interesting and novel features of models with two nonlinearities is the cross-coupling dynamics of the two nonlinearities ${ }^{1}$. In the previous chapter, crosscoupling dynamics was represented analytically as a frequency shift because the propagator was taken to be real. In fact the cross-coupling dynamics has two parts: the nonlinear frequency shift from the cross terms of equation (4.10) multiplied by the real part of the inverse propagator $\Delta \omega_{\mathrm{k}}{ }^{-1}$; and a dynamical effect from the cross terms multiplied by the imaginary part of the inverse propagator. The dynamical effect changes the transfer dynamics and therefore changes the saturated (or instantaneous) spectrum. This can then change the spectrum weighted average $\mathbf{k}$. All of which can, in principle, affect quantities such as transport. The frequency shift has a more subtle consequence. Through the propagator it also modifies the transfer dynamics, moreover, there can be an effect on the mode stability (linear stability). This change of mode stability is an outgrowth of the inertia of the adiabatic electrons which give rise to a frequency change in the form of a $\left(\omega-\omega^{*}\right)$ term in the dispersion relation. The model used previously, which was a one-field model, has the linear growth spectrum $\left(\gamma_{\mathrm{k}}\right)$ fixed. This type of model does not permit the frequency shifts to impact the linear stability. In order to investigate the ramifications of such a feedback, a two-field model for dissipative trapped electron modes is used ${ }^{2}$. In this model, two fields are evolved, the potential $(\phi)$ and election density $\left(\mathrm{n}_{\mathrm{e}}\right)$, with all of the quadratic nonlinearities intrinsic to this problem. This model permits the investigation of the dynamics in both the strongly dissipative regime, where the one-field model is valid, and the collisionless regime. The two main benefits of using a two-field model are the ability to investigate the interplay between the frequency shifts and the linear growth spectrum, and a thorough exploration of the transport properties of this type of mode. In the one-field model the phase relation between $n$ and $\phi$ is set and therefore, the transport $(\mathbf{B l b c k}(\mathbf{v n})=$ $\langle\nabla \phi \times \mathrm{z} n\rangle$ ) is an artificially constrained quantity. In the two-field model, it is possible to examine the evolution of the flux under the nonlinear processes that govern the correlation of $\mathrm{n}$ with $\mathrm{v}$ and determine how the individual modes or regions of modes contribute to the net flux. For example, do some regions in $\mathbf{k}$ space contribute an inward flux while others give an outward flux?

As before, a model with distinct subranges will be investigated ; the long wavelength extreme of the spectrum is dominated by the ExB nonlinearity while at the high $\mathrm{k}$, shorter wavelength extreme is dominated by the polarization drift nonlinearity. The previous computational and analytic results from the one-field model confirm the existence of the two extremes, but also point out the importance of the intermediate regime. In this region of $\mathbf{k}$ space, neither the $\mathrm{E} \times \mathrm{B}$ nor the polarization drift nonlinearity dominate completely and the interplay (cross-coupling) becomes very important. The earlier work with the one-field model showed that in the long wavelength subrange there exists a direct, non-local, anisotropic cascade of energy to high $\mathbf{k}_{\mathbf{y}}$ - In the short wavelength (polarization drift dominated) subrange, a dual cascade analogous tc \%-Dimensional Navier-Stokes was found with an inverse cascade of energy to low $\mathrm{k}$ and a direct cascade of enstrophy (mean squared vorticity) to high $k$. The cascades due to the polarization drift nonlinearity were largely local 
and isotropic in nature, in agreement with the well known Navier-Stokes dynamics. In the crossover region the dynamics are mixed but not a superposition of the two extremes: the net dynamics tends to be a more isotropic, yet a non-local direct cascade of energy to high $\mathbf{k}$ remains in force. In addition to the change in transfer dynamics, a large frequency shift, resulting from the imaginary part of the cross-coupling, is found.

It is important to keep in mind that many or most physical systems contain multiple nonlinearities and while it is true that one nonlinearity may dominate the dynamics in a given region of $\mathbf{k}$ space, at some point a region may exist in which multiple nonlinearities of comparable importance co-exist. In some systems, DTEM for example, the dynamics at the extremes, where just one nonlinearity dominates, conspire to increase the importance of the crossover region. In the case of DTEM, the EXB nonlinearity tends to transfer energy into the crossover region from low $\mathbf{k}$ while the polarization drift nonlinear inverse cascades energy into the crossover from high $\mathbf{k}$. These combined cascades increase the total energy in the crossover region and act to make the crossover dynamics very important for the total system dynamics.

In this work many of the techniques used in the earlier studies are applied to determine if the same dynamics hold in the more complete system. The spectral flow diagnostic is used to describe the transfer dynamics of the two nonlinearities, i.e., whether it is, local or non-local, direct or inverse, and isotropic or anisotropic. Additionally, the frequency and $\mathrm{k}$ spectra are analyzed temporally, to investigate secular and quasi-periodic variations. As before, the computational work is done using a spectral code containing up to 41 by 41 modes. Two general types of simulations are performed: 1) scanning of collisionality while holding other factors roughly constant; and 2) scarning of the ratio of relative strength of nonlinearities while holding the collisionality ( $v$ ) fixed. In both cases some adjustment of damping is made in order to investigate the effect of varying saturation levels. These two classes of simulations permit the investigation of a variety of phenomena In the first case one effectively scans the growth rate and the importance of having twofields. In the high $v$ region this permits verification of the results of the one-field model while still including a much more realistic growth spectram. In the second set of simulations it is possible to study the effect of the cross-coupling terms on growth rates and spectrum shapes in a self consistent way. Here the focus is on the average and instantaneous rates of energy extraction, spectrum shape and transfer dynamics.

The main results in this chapter are now summarized. It is found that while the results from the one-field model can be reproduced in the high $v$ regime of the two-field model, there are additional new phenomena intrinsic to multi-field models. Despite the fact that in the two-field model the nonlinearities are in different fields $(E \times B$ in the density field and polarization drift nonlinearity in the potential field) the model behaves very much like the one-field model when $v$ is large. The nonlinearities cascade in the same fashion with the $\mathrm{E} \times \mathbf{B}$ nonlinearity transferring energy nonlocally to high $\mathbf{k}_{\mathbf{y}}$ in the region it dominates. Once again it is in the cross over region that the dynamics are different. The total (net) transfer in the cross over region is dominantly a direct cascade to high $\mathbf{k}$. However it is more isotropic than when the ExB nonlinearity acts alone and it is more non-local than when the polarization drift nonlinearity acts alone. As before, in this subrange, there is marked evidence of a large change in the frequency dynamics. In addition to this expected dynamics, a variety of new phenomena are found. The most significant of these is the nonstationary nature of the saturated quantities. In general, in turbulent systems it is 
assumed that once saturation is reached quantities such as mode amplitude, frequency spectrum, transport and $\mathrm{k}$ spectrum are constant when averaged over a few eddy tumover times. This, in some sense, is the definition of a saturated steady state. In the present system something very different is observed - large-scaie global fluctuations. These fluctuations in total energy, enstrophy, and flux are characterized by both their large ( $>30 \%$ ) amplitude and their long period $\left(>5 \tau_{\mathrm{e}}\right.$ ). During the global fluctuations most of the local (local in $\mathbf{k}$ space) quantites undergo marked change. The $\mathbf{k}$ spectrum fluctuates, the frequency spectrum fluctuates, the transfer rates fluctuate, and the transport fluctuates. Distinct phases of these fluctuation cycles are identifiable. These phases can be broadly defined as the preliminary phase (at the bottom of a cycle), growth phase on the up cycle of the fluctuation. turnover phase (at the peak of a cycle), and the crash phrase (on the declining side of a cycle). The $\mathbf{k}$ space regime in which these fluctuations are most visible is the crossover region where both nonlinearities are comparable. During the cycle the frequency spectrum for a mode in the center of the crossover range undergoes a metamorphosis from no shift to upshift to downshift while the energy in those modes grows, then transfers out, and finally crashes. It must be kept in mind that if time histories are averaged over many eddy turnover times $(>20)$, these fluctuation can be lost or overlooked.

The organization of this chapter is as follows: The model and its properties are presented in section II along with the reduction to the one-field model. Section III presents a brief heuristic explanation for the cycling fluctuations. Section IV has the results of the two-field model. Finally, section V presents conclusions.

\subsection{Basic Model and Properties}

In this section the basic model is briefly presented and some of its main properties discussed. This is done in the context of comparing and contrasting the present model with the one-field model of the same system. This analysis examines the linear structure, integral invariants, and the fourier transformed mode equations.

The model utilized for this study is a set of trapped particle fluid equations. This model couples the dynamics of collisional trapped electrons with hydrodynamic ions through the quasineutrality condition. The derivation of this equation and a discussion of its details and limitations have been presented elsewhere. In previous work, the inertial response of the electrons was neglected by considering only the most dissipative extreme of collisionality. Here, the electron nonlinearity and inertia terms are included, yielding the model equations:

$\left(1-\sqrt{\varepsilon}-\rho_{\mathrm{s}}^{2} \nabla^{2}\right) \frac{\partial \phi}{\partial t}+V_{D} \frac{\partial \phi}{\partial y}-\sqrt{\varepsilon} V_{D}\left(1+\infty \eta_{e}\right) \frac{\partial \tilde{\pi}_{e}}{\partial y}-\sqrt{\varepsilon v_{e}} \overline{\mathbf{n}}+\sqrt{\varepsilon v_{e}} \phi$

$+\rho_{s} C_{s} \nabla \phi \times z \cdot \nabla \rho_{s}^{2} \nabla^{2} \phi+\mu \nabla^{4} \phi=0$

and

$\frac{\partial \tilde{n}_{e}}{\partial t}+v_{D}\left(1+\alpha \eta_{e}\right) \frac{\partial \tilde{n}_{e}}{\partial y}+v_{e f f} \tilde{n}_{e}-C_{s} \rho_{s}^{3} \nabla \phi \times z \cdot \nabla \tilde{n}_{e}-v_{e f f} \phi=0$,

where $\tilde{\mathrm{n}}_{\mathrm{e}}=\mathrm{n}_{\mathrm{e}}+\phi$ is the fluctuating electron density plus the fluctuating potential, $\mathrm{V}_{\mathrm{D}}=$ (cT $\left.T_{e} / e B\right) L_{n}^{-1}$ is the diamagnetic drift velocity, $D=\varepsilon^{1 / 2} V_{D}{ }^{2}\left(1+\alpha \eta_{e}\right) / v_{e f f, e}$ is a negative 
diffusivity describing the destabilization of DTEM modes by electron collisions, $\varepsilon$ is the trapped electron fraction, $\eta_{\mathrm{e}}=\mathrm{d} l n \mathrm{~T} / \mathrm{d} l n \mathrm{n}$ is the electron temperature gradient parameter, $v_{\mathrm{i}}$ models long wavelength collisional damping, $v_{\text {eff,e }}=v_{e} / \varepsilon, \mu$ is the coefficient of the hyperviscosity introduced to model strong damping at high $k, L_{n}$ is the density gradient scale length, $\rho_{s}=\left(c T_{d} / e B\right) / C_{S}$ is the ion gyroradius evaluated at the electron temperature, and $C_{S}$ $=\left(T_{e} / m_{i}\right)^{1 / 2}$ is the ion sound speed. The substitution of $\tilde{n}$ for $n_{e}+\phi$ is for computational simplicity. The primitive equation for $\mathrm{n}_{e}$ has a time derivative of $\phi$ in addition to the time derivative of $n_{e}$, making the use of explicit solvers impossible. This change in no way alters the dynamics of the problem and was made at this stage for consistency with the computational work. In contrast with the previous work (chapter 4), the linear polarization drift term responsible for linear dispersion has been included in Eq. (5.1). The basic mechanisms governing transfer and the concepts describing this process are however unaltered. Indeed, numerical solutions with and without the linear polarization term included were found to be qualitatively the same for spectral ranges studied herein.

The nonlinearity appearing in Eq. (5.2) is the $\mathrm{E} \times \mathrm{B}$ nonlinearity, arising from $\mathrm{v}_{\mathrm{E}} \cdot \nabla$ $\tilde{n}_{i}$, where $v_{E}=-\left(c / B_{0}\right) \nabla \phi \times z$ is the $E \times B$ drift. The nonlinearity which appears in Eq (5.1) is the polarization drift nonlinearity, and arises from $n_{0} \nabla \cdot v_{p}^{(1)}$, where $v_{p}^{(1)}=B_{0}$ ${ }^{1}\left(m_{\mathrm{i}} \mathrm{c} / \mathrm{e}\right) \mathrm{z} \times \mathrm{v}_{\mathrm{E}} \cdot \nabla \mathrm{V}_{\mathrm{E}}$ is the nonlinear polarizaticn drift. The $\mathrm{E} \times \mathrm{B}$ nonlinearity requires a nonadiabatic electron response (provided by the trapped electrons), whereas the polarization drift nonlinearity derives from the ion polarization drift

From Eqs. (5.1) and (5.2), it is again apparent that, by virtue of its additional spatial derivative, the polarization drift nonlinearity dominates the $\mathrm{E} \times \mathrm{B}$ nonlinearity at very shor wavelengths. The converse holds at long wavelengths. The nominal crossover point is given by the wavenumber at which the two nonlinearities are equal. This crossover point is however also dependent on the effective electron collisionality $\left(v_{\text {eff.e }}\right)$. Assuming rough isotropy, so that $\nabla_{\perp} \approx \partial / \partial y$, this wavenumber is given by $k \rho_{s}=\delta=C_{s} / L_{n} v_{e f f . e} \equiv k_{0} \rho_{s}$. Because the nonlinearities are characterized, not by a single spatial scale, but by a triad interaction consisting of three waves of differing wavelengths, it is more realistic to identify a region, centered about the crossover wavenumber, in which the two nonlinearities are comparable, rather than to speak of a single wavenumber at which the two are equal. It is within this region that the cross-coupling dynamics are dominant.

Due to the presence of the EXB ronlinearity, a single quadratic invariant, the energy, is admitted by this system in the absence of driving and damping. As previously discussed. the $\mathrm{E} \times \mathrm{B}$ noniinearity in isolation transfers energy to short wavelengths, given a spectrum which is peaked at long wavelength or flat, and therefore drives robust production of enstrophy ${ }^{2}$. This term is present even in spectral ranges where the polarization drift nonlinearity dominates $\left(k>k_{0}\right)$. Consequently, enstrophy is not conserved even when the EXB nonlinearity is weak compared to the polarization drift nonlinearity. However, in such a case, the EXB nonlinearity accounts for proportionately less of the total energy transfer. Because enstrophy production is tied to energy transfer by the ExB nonlinearity, it can be expected that the importance of enstrophy production in the cascade dynamics diminishes for $k>k_{0}$. For $k<k_{0}$, the eddy turnover rate is controlled by the dominant EXB nonlinearity and can be expected to be comparable to the enstrophy generation rate. For $k>$ $k_{0}$ the eddy tumover rate is controlled by the larger polarization drift nonfinearity, while the enstrophy generation rate is tied to the weaker ExB nonlinearity. Consequently, there will be litule enstrophy generation on the nonlinear interaction or nonlinear transfer timescale.

As described in chapter 2, energy transfer that is anisotropic and nonlocal in wavenumber space is a robust feature of the $\mathrm{E} \times \mathrm{B}$ nonlinearity, but at variance with the conventional picture of the cascade process. On the other hand, the polarization drift nonlinearity, investigated in chapter 3 , is of the same form as the advective nonlinearity of the vorticity evolution equation of Navier-Stokes turbulence. Therefore, as expected, the 


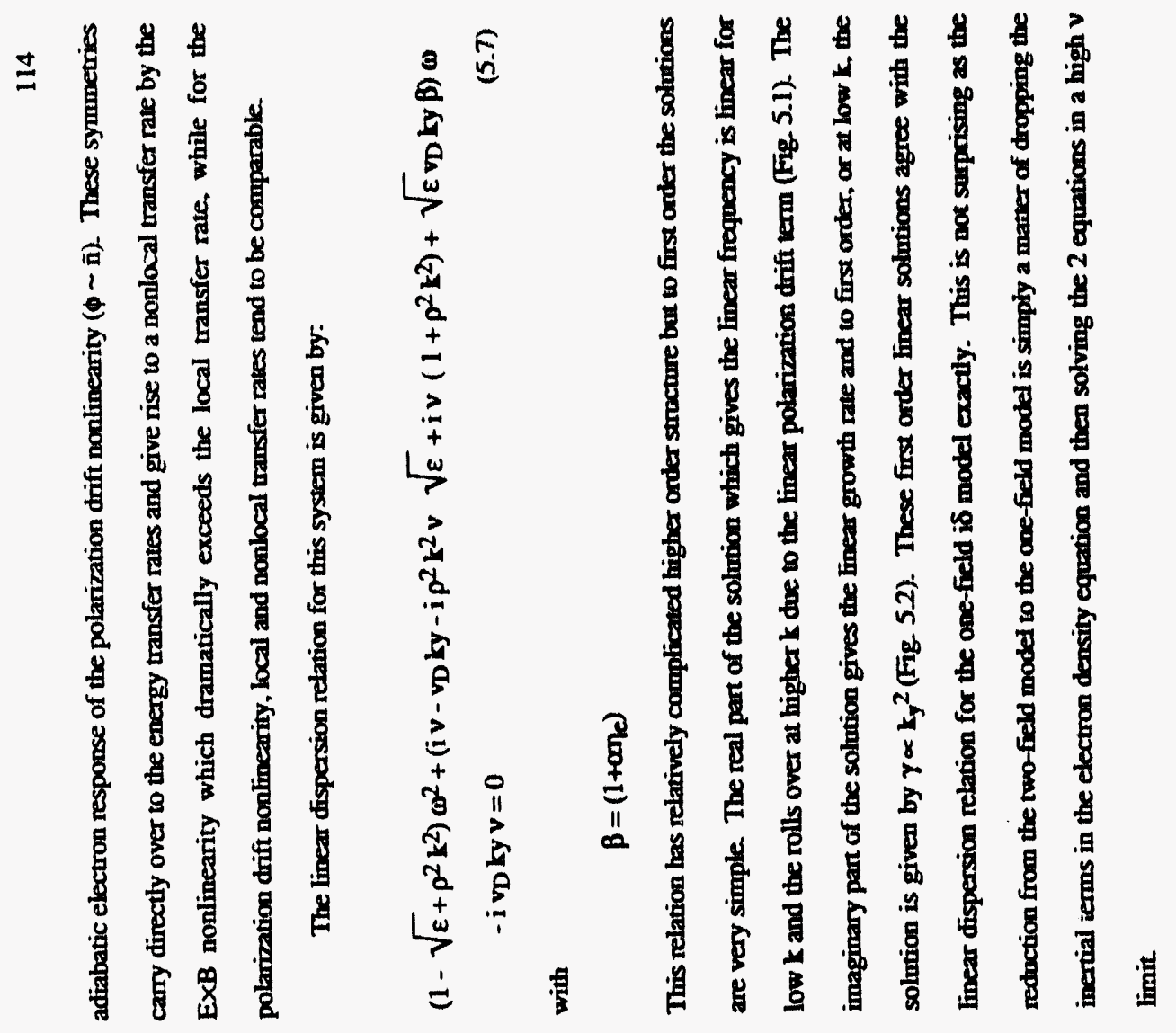

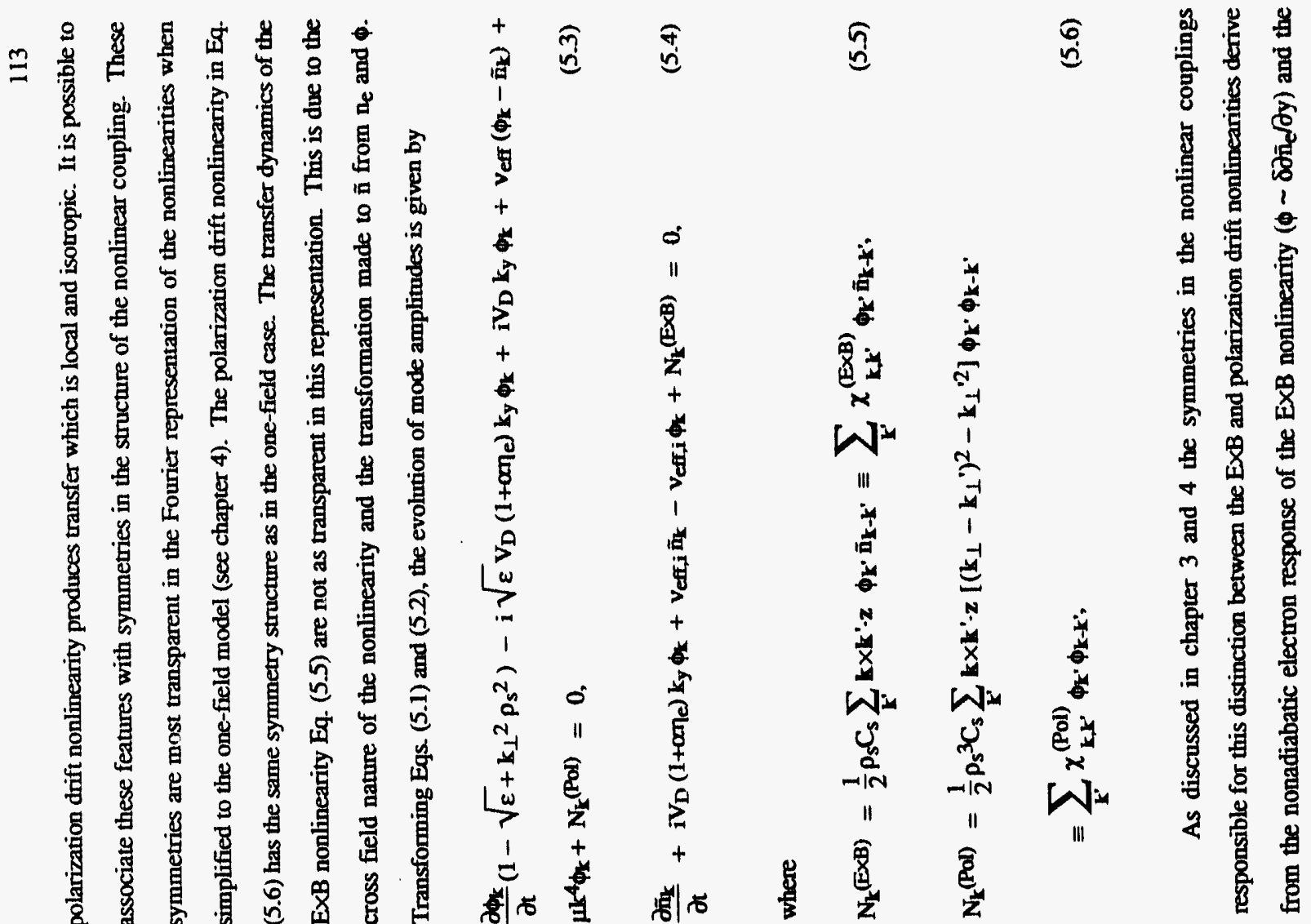




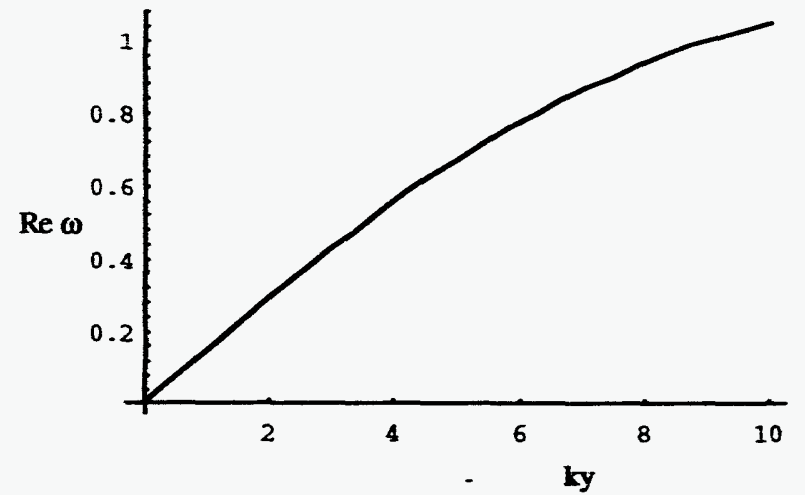

Fig. 5.1 The real part of the dispersion relation. This gives the frequency spectrum as a function of $k y$.

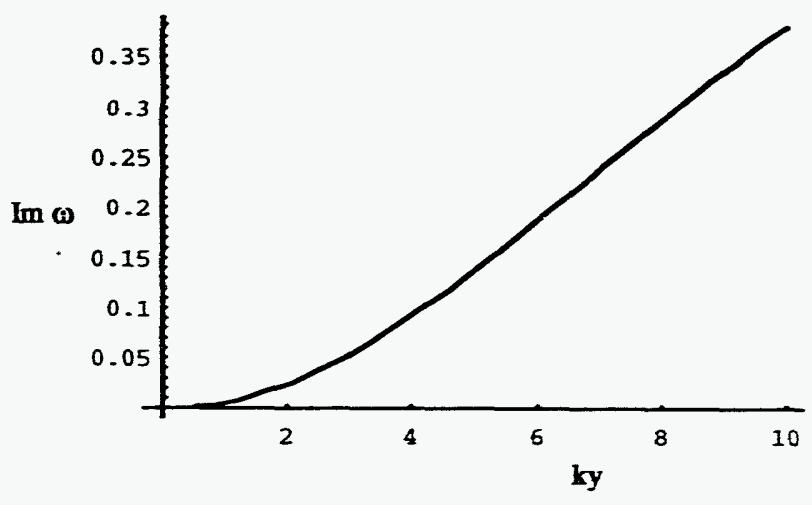

Fig. 5.2 The imaginary part of the dispersion relation. This gives the growth rate as a function of ky

\subsection{Henristics of the Cycting}

Relaxation oscillations are relatively ubiquitons in nature, existing in systems as simple as a bow drawn over a violin string to systems as complex as sawreeth in a fusion device. In most of these cases there is a competition between forces driving the system away from its preferred state and restoring forces. In addition to these two competing forces some form of inertia is noeded to take the system through and beyond its equitibrim. In the case of the violin string the oscillation is driven by the difference between the static and the dynamic coefficients of friction and the inertia of the string. While the cycles in the two-field model ane not as simple as the violin string it is instructive to go through a few scenarios for the mechanism behind the two-field oscillations. First, two simple easily pictured mechanisms will be outinod; then, a theird more realistic mechanism combining the first two will be curlined. The first scenario is a simple feedbect of the froquency shift on the linear growth rase with overshoot due to spectrum inertia In this case, the system starts from small amplitude. From closure calculations the frequency shift is found to be amplinde dependent, $\left(\omega_{s} \propto \sum_{k^{\prime}} k_{y}\left(2 k_{y}{ }^{2}-k_{y}{ }^{2}\right) k_{z} \cdot l^{2}\right)$, which suggeses that at low amplitude there is Fittle or no frequency shift which allows the linear growth to proceed. As the modes gain in amplitude the frequency shift becomes more pronounced. Since the finear growth rate is $\gamma$ $\alpha\left[\omega_{*}\left(1+\alpha \eta_{e}\right)-\omega\right]$, when $\omega>\omega_{*}\left(1+\alpha \eta_{e}\right)$ the growth becomes negative and the mode is stabilized. Therefore at some point in the growth the mode amplitudes become large enough to stabilize the mode and the growth ends. The mode then loses energy unil the amplitude is small enoagh to tum off the frequency shift which in turn reactivates the 
growth rate. The spectrum inertia is required to force the mode amplitudes to overshoot the amplitude which would cause a frequency shift giving marginal stability. The system could then undergo stable oscillations around the marginally stable amplitudes.

The second mechanism is similar to the first except that the frequency shift is replaced by a change in the dynamics due to the cross-coupling, ie., $\chi_{k, E^{\prime}}^{(E \times B)} \chi_{k-k^{\prime}+k^{\prime}}^{(\text {Poln }}$ $\Delta a^{-1}$. In this case as the system grows from small amplitude the dynamics of the transfer are dominated by the individual nonlinearities. This is becanse the cross-coupling dynamic shift is also dependent on the amplitude. As the most unstable modes (assumed to be the middle $\mathbf{k}$ modes near the crossover) grow higher than the surrounding modes the crosscoupling dynamics shift starts to dominate over the stand:rd dynamies. This allows energy to be transferred rapidly out of these high amplitude modes to the damped modes at either end of the $\mathbf{k}$ spectrum. As the energy is transferred out, the mode energy decreases eventually reaching the level at which the cross-couping dymamics are less important than the dynamics of the individual nonlinearities. At that point the mode starts linearty (and nonlinearty) growing again. Once again the spectrum (or transfer) inertia is relied on to force an overshoot of the mode amplitudes allowing the cycling to occur.

The final scenario combines the first 2 and appears more realistic for this model Again, the system starts from low amplitude, as the amplitudes grow the frequency shift grows once again having a feedbact effect on the growth rate. The shift in transfer dynamics becomes important at a larger amplitude becanse it depends on the nonresonant part of the propagator (ie, Im $\Delta \omega$ ) and is, as such, dependent on the frequency shift. As the frequency shift becomes large enough to stabilize the mode, the transfer dynamics shift rapidly moves the energy out of the crossover modes and into new modes. This forces the amplitude of the new energy bearing modes to grow rapidly causing them to undergo simitar shifts in transfer dynamics, eventually transferring the energy to dissiparive modes. While the energy is being cascaded to dissipative modes the energy in the linearly most unstable modes falls well below the marginal stability amplitude doe to the combined effects of the frequency shift induced stabilization and the shifted transfer dynamics. The cycle then restarts. Many of the elements in this mectanism can be inferred from the dingnostics in the compucational studies, as will be shown in the next section.

\subsection{Computational results}

In this section the computional results from the two-field model anc cescibed. in the two-field compuntions, as with the one-field wort, a number of compuntional diagnostic tools were used. The nonlinear transfer diagnostic is based on the energy evalution for an individual model $k$. The equations for the energy evolution awe

$$
\frac{(1-\sqrt{e})}{2} \frac{\partial h_{l} l^{2}}{\partial t}-\sqrt{e v h_{k}} l+\sqrt{e} v\left|h_{l}\right|^{2}=T_{k} \text { (Pal) }
$$

and

$$
\frac{1}{2} \frac{\partial n_{k} l^{2}}{\partial t}+v m_{1} l^{2}-v n_{k} n_{k} l=T_{k}(E \times B)
$$

with

$$
T_{k}^{(P o l)}=c_{s} P_{s} \operatorname{Re} \sum_{K}\left(k \times k^{\prime}-z\right)\left(k-k^{\prime}\right)^{2} n_{k}, n_{k-k^{\prime}} n_{k}^{*}
$$

and

$$
T_{k}(E \times B)=c_{s} p_{s} \operatorname{Re} \sum_{Y}\left(k \times k^{\prime} \cdot z\right) \phi_{k} \cdot B_{k-k} \cdot \varphi_{k}^{*}
$$

Hexe $T_{k}(E \times B)$ is defined as the $E \times B$ nontinear transfer rate and $T_{k}(P o l)$ is we polarization drift nonlinear transfer rate. If $T_{\underline{k}}$ is negative (positive) that implies nonlinear 
transfer out of (into) the mode $k$. For simplicity of data storage this information is not kept for all modes individually; instead, the flow from (to) bands in the $k_{x}$ and $k_{y}$ directions are determined. The flow is defined as local if the interacting modes are within twl modes of $k$ where $m$ is taken to be between $1 / 3$ and $1 / 2$ of the total $k$ spectrum size. Nonlocal flow is all flow not within the region defined by $m$. In most cases $m$ is chosen to be large in order to insure that the local/nonlocal differentiation is equivalemt to local in the Kolmogorov inertial range sense.

In addition to the mode energy/enstrophy transfer diagnostic. global energies $\left(\left.\operatorname{mos}_{k}\right|_{\text {tor }} ^{2}\left|\phi_{k}\right|^{2}\right.$ tot $\left|m_{k}\right|^{2}$ tot $\left.+\left|h_{k}\right|_{\text {tot }}^{2}\right)$ and flux $(\langle n \nabla \phi\rangle)$ are tracked. Whike the global quantities are useful, and in fact first showed the cycling, the local (loca being defined as consisting of a single mode) quantities are more instrictive and are therefore followed in detail. Mode energy, flux for a given $\mathbf{k}$ and frequency spectra for individual modes are all analyzed and used in conjunction with the transfer rates to investigate the dynamics within each field as well as the cross correlations tetween fields. Finally, it has been found to be very useful to animate sequences of 2-D $\mathbf{k}$ spectra and real space isocontours. These animation sequences often give an idea as to which dynamics dominate in a given temporal region

With the exception of runs done for the purpose of testing the code, all of the computation was done with driving and damping turned on. Typically the linear growth spectrum was peaked at intermediate $\mathbf{k}$ with viscosity (or hyperviscosity) decreasing the growth rate at higher $\mathbf{k}$. This results in a growth rate $(\gamma)$ that is negative at lowest and highest wavenumbers and positive at intermediate $k_{y}$ with $k_{x}=0$. When the simulations are started from infinitesimal initial amplitudes, a linear growth regime is observed followed by the onset of nonlinear interactions which reduces the net growth ate (linear phus nor'inear) of the most limearly unstable modes and increases the net growth rate of the less unstable modes. As is Jmmon with turbalent simulations, saturation does not occur if the total growth rate, $\left(\boldsymbol{Y}_{T}=\sum_{\mathbf{K}} \boldsymbol{Y}_{\mathbf{k}}\right)$ is positive, while saturated "steady" states whose saturation amplitude is sensinivety dependent on $\gamma_{\mathrm{T}}$ exist for negative $\gamma_{\mathrm{T}}$

In the fimit of large $v(v>>)$ the model is reducible to the one-field model. Consistent with this, the dymanics of the compotational simulations of the two-field model are virually indistinguishable from the cone-field resolks in the high $v$ limit $\mathrm{m}$ the regioa of k space in which the polarization drift nonlinearity dominates the flow is largely bocal in matre and exhibits a dual cascade. The spectrum is dominantly peaked at low $\mathrm{k}$ so the encrgy bearing modes are below the driving or maximally unstable $k$ There is very firte or no frequency shift for the modes stroagly domimated by the polarization drift montincarity. howeverit is on the polarization drift dominated side of the crossover region that the froquency shift reaches its maximum. In the region dominated by the ExB nonlinearity the transfer is predominately poniocal and anisotropic, and energy is diwectly transferred to high $k_{y}$ modes. The transfer in the $k_{x}$ dinection is ako direct but more local bi this regime there is no appreciable frequency shift and the dymamics of the ExB noalinearity dominate the system dynamics. The $k$ spectrun in the EXB domimated region is anisocropic with the peal at the most unstable modes. This leads to a spectrum platean which is broesend in the $k_{y}$ firection and is much flatter then the spectrum in the polarization drift nonlinearity dominated region.

When $v>\omega$ the dynamies of the ExB and polarization drift subranges remains nearly identical to the results from the one-field model; however, new phenomena are 


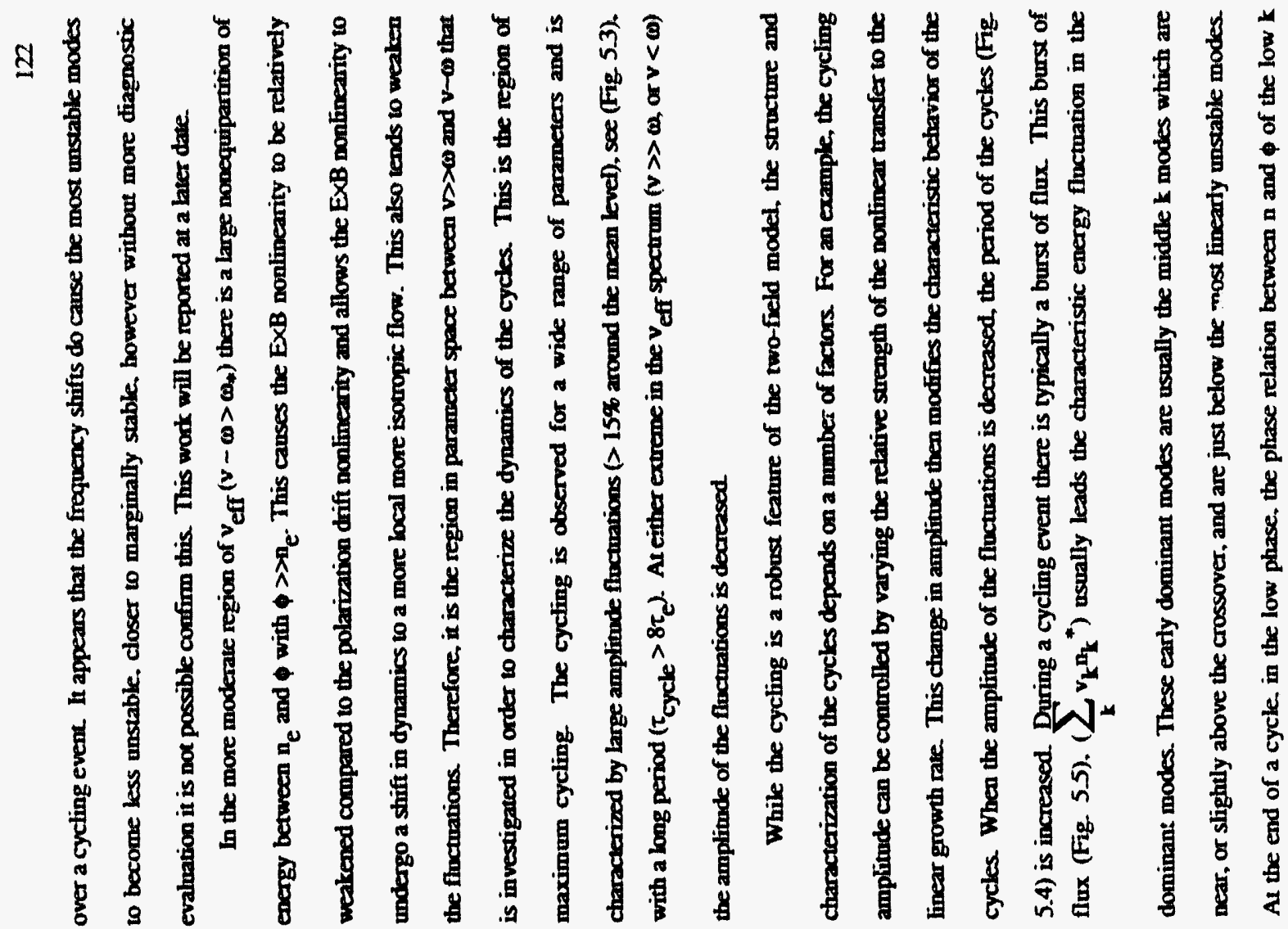

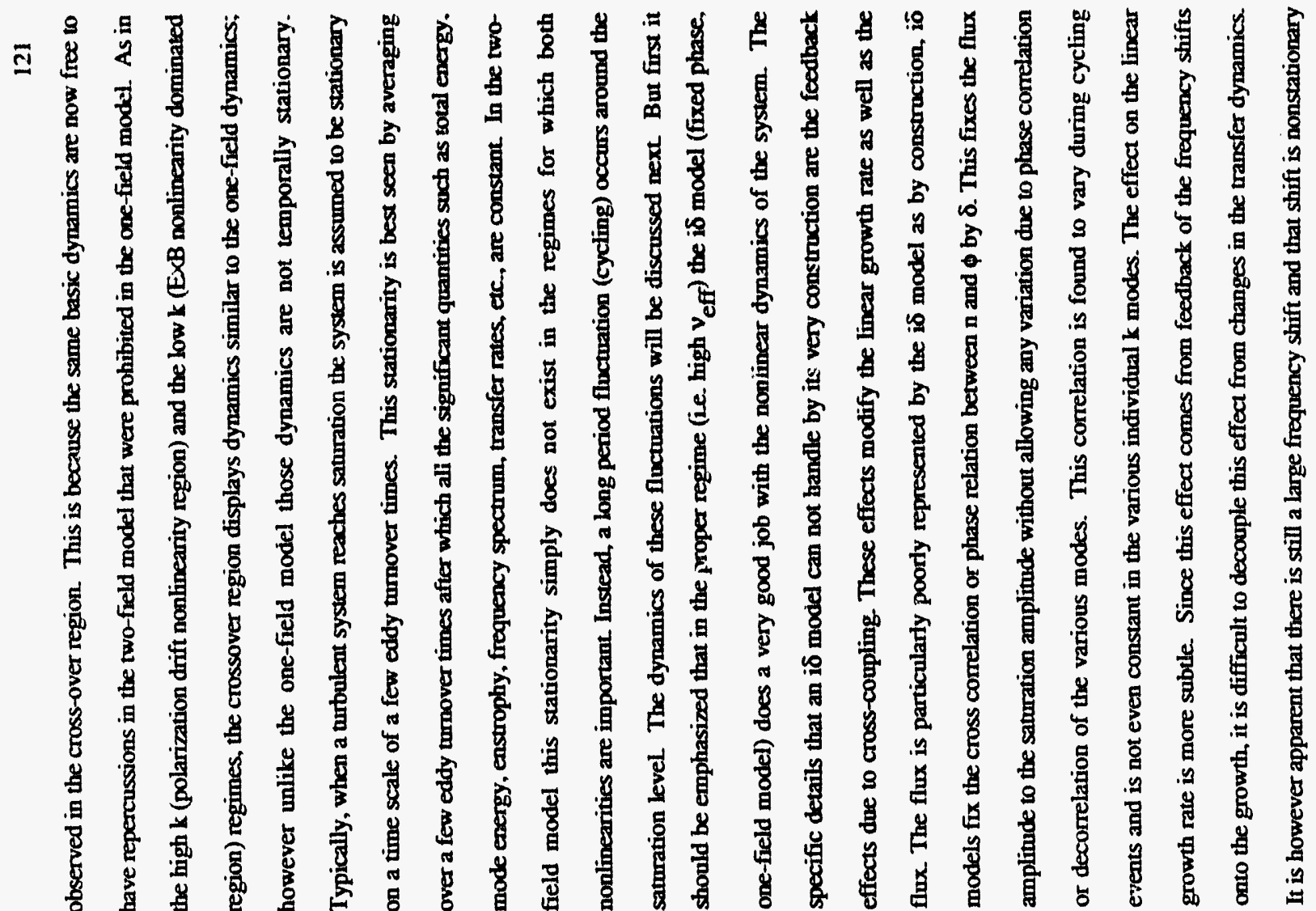


I
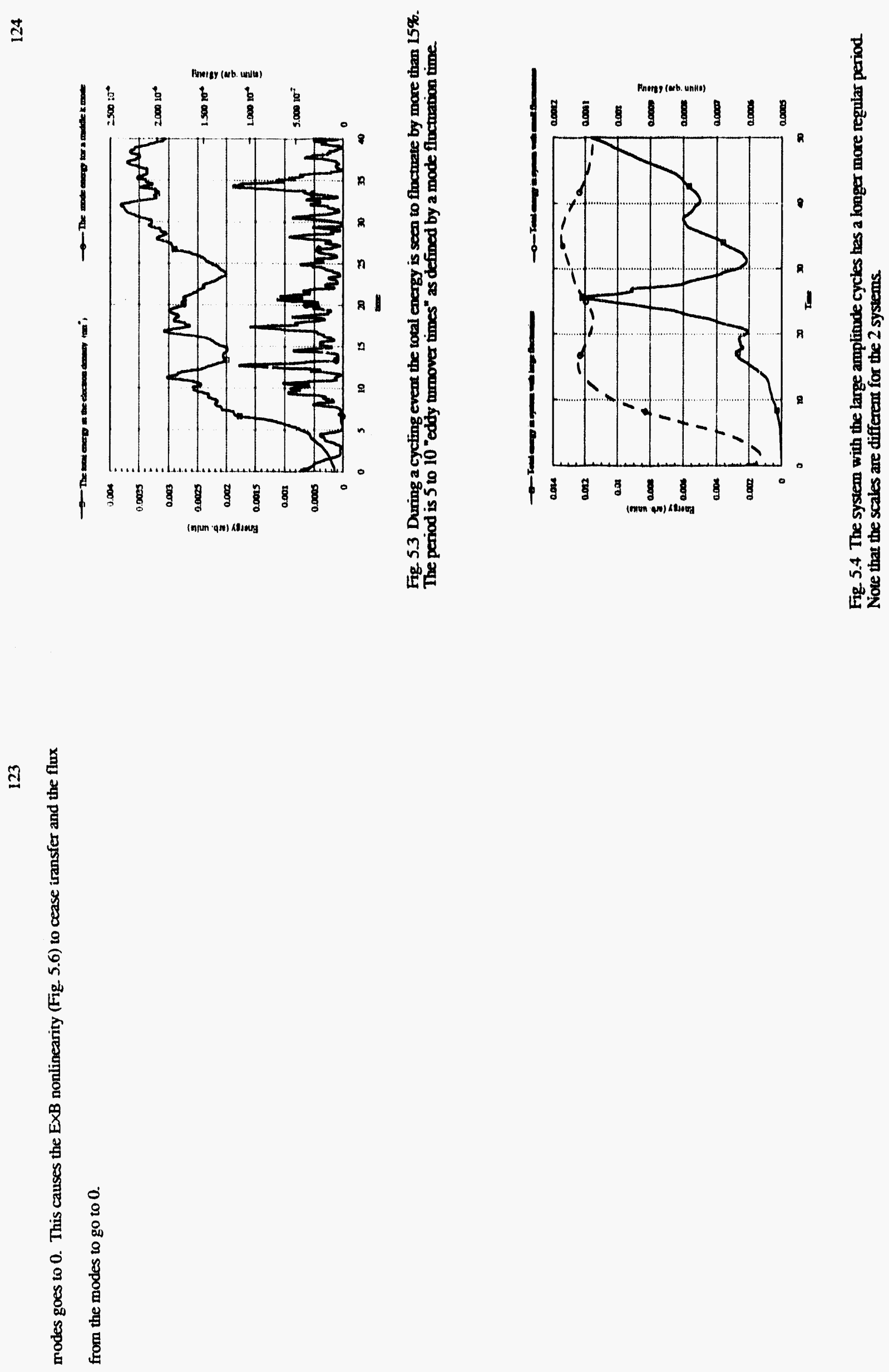


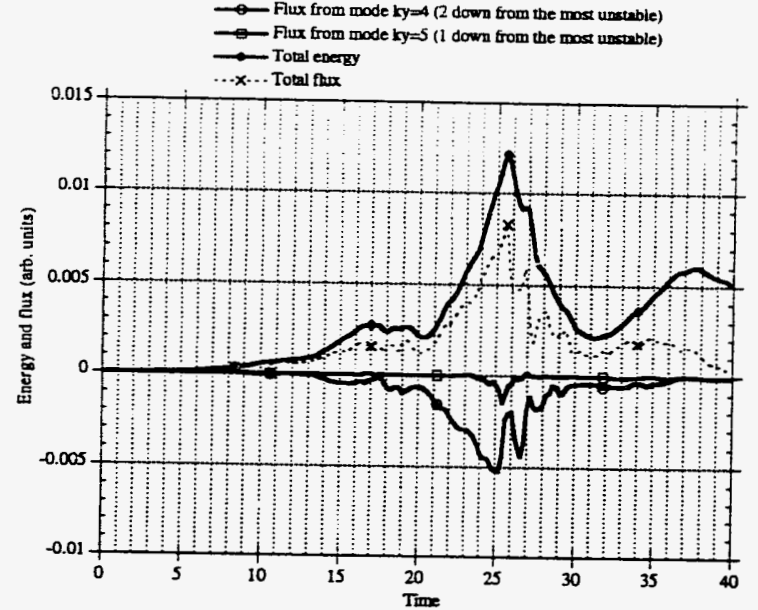

Fig. 5.5. The flux from the 2 most active modes "bursts" coincident with the fluctuation. The burst in the most active mode leads the fluctuation by a small phase.

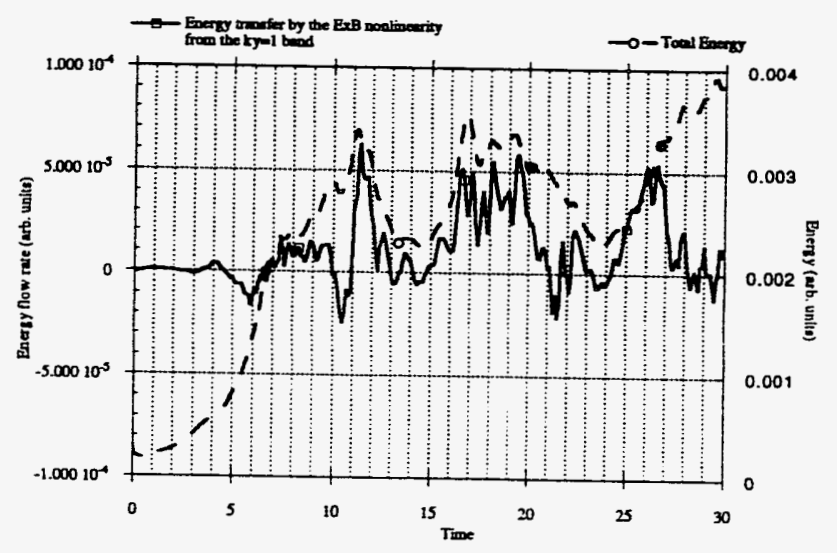

Fig. 5.6 Total energy evolution with the ExB flow evolution during fluctuations The ExB transfer goes to zero at the end of a cycle then starts again. In fig 5.5 the same behaviour in shown for the flux from a mode
These occur both $\left\langle\mathrm{v}_{\mathrm{k}^{n_{\mathrm{k}}}}{ }^{*}\right\rangle$ (flux) and $\nabla \mathrm{n} \times \mathrm{z} \cdot \nabla \phi$ (ExB nonlinearity) are maximum when the phase shift between $\mathbf{n}$ and $\phi$ is $\pi / 2$, and zero when $\mathbf{n}$ and $\phi$ are in phase. The phase is then rescrambled on a nonlinear time scale, i.e., approximately one eddy turnover time. The flux and nonlinear transfer then resume.

In the course of a cycle the modes that undergo the most significant changes are the middle ky modes early to midway through a cycle and then low in $\mathbf{k}_{\mathbf{x}}$ propagating to high $\mathbf{k}_{\mathbf{x}}$ at the end of a cycle. During such an event the frequency spectrum for the middle to low middle $\mathbf{k}_{\mathbf{y}}$ modes undergoes large changes. It is difficult to resolve frequency spectrum fluctuations on the time scale of a cycling fluctuation because a cycle period is of order 10 eddy turnover times. This means that each phase of the cycle lasts $-3 \tau_{e}$ and even then may not be stationary. Nevertheless it is possible to extract some useful spectra assuming the existence of two, three, and four distinct phases in the cycle, and using conditional sampling techniques to extract spectra. These simply assume that one cycle is like another in terms of the relationship between the frequency spectra and the phase of a cycle. Combining multiple partial cycles allows one to create a record of 10 s of eddy turnover times for frequency analysis. These low middle ky modes show (Fig. 5.7) a frequency downshift early in the cycle followed by a region of little or no shift, followed by a large upshift. These shifts would be consistent with the suggestion that the linear growth is reduced at the top of the cycle and is then turned back on at the bottom. It is worth noting here that by averaging over a few cycles $\left(-30 \tau_{\mathrm{e}}\right)$ one would miss the nonstationarity of the frequency spectrum and would simply see a broadened frequency spectrum. Unfortunately the explanation of the cycling is probably not as simple as the mechanism which simply has the frequency shift 
$\stackrel{\infty}{\infty}$
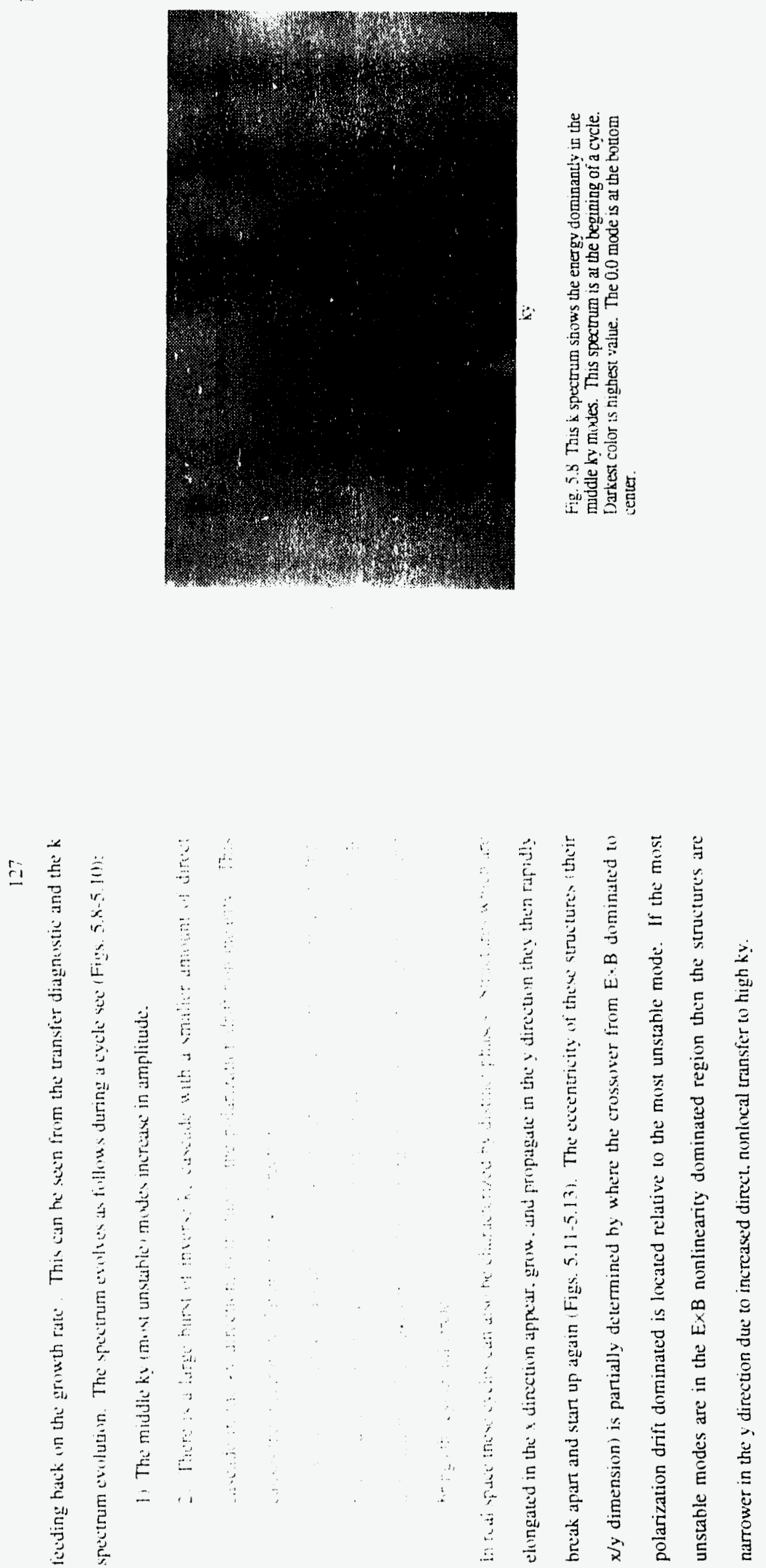

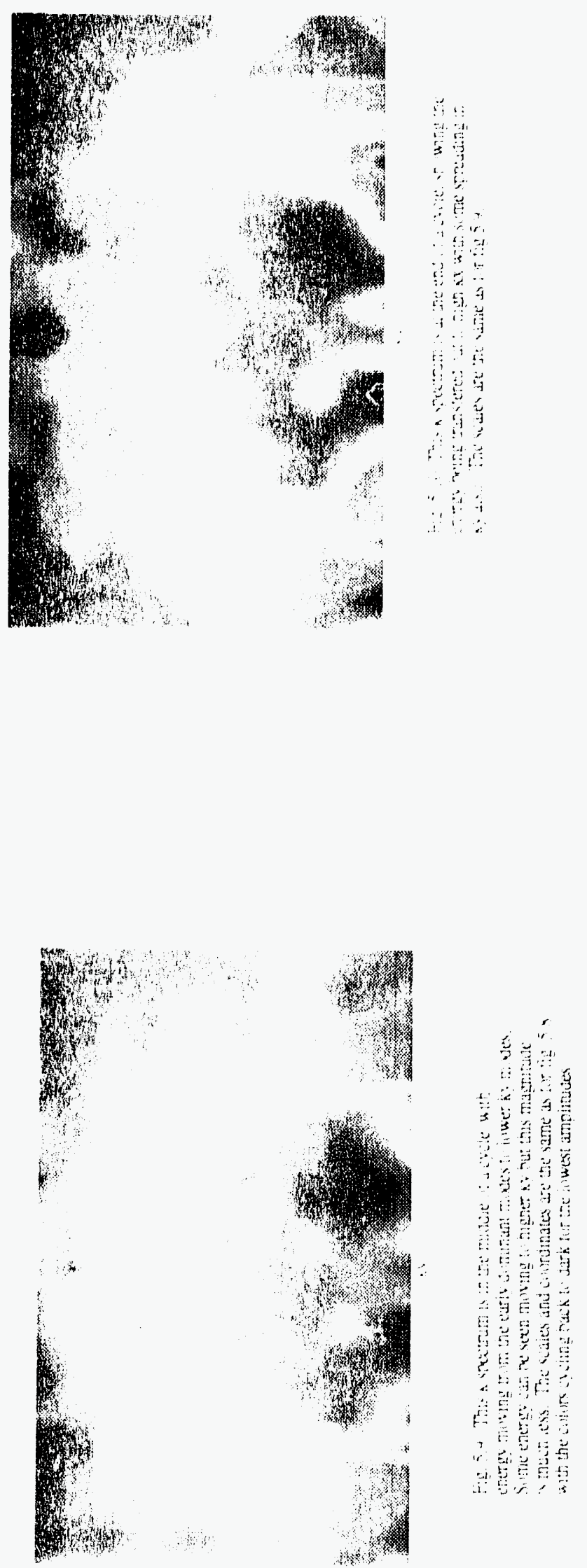
్ㅣ

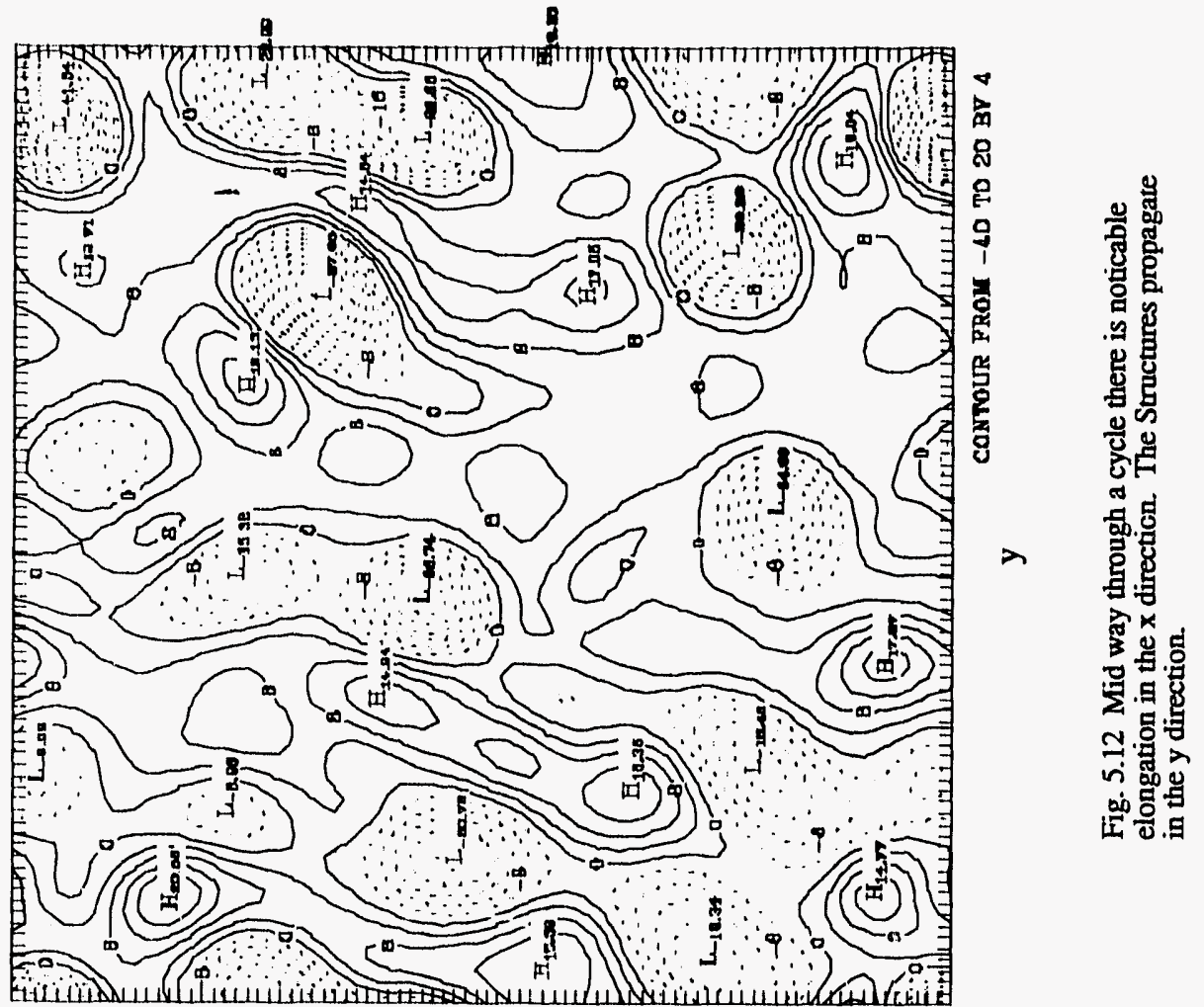

$\overline{-}$

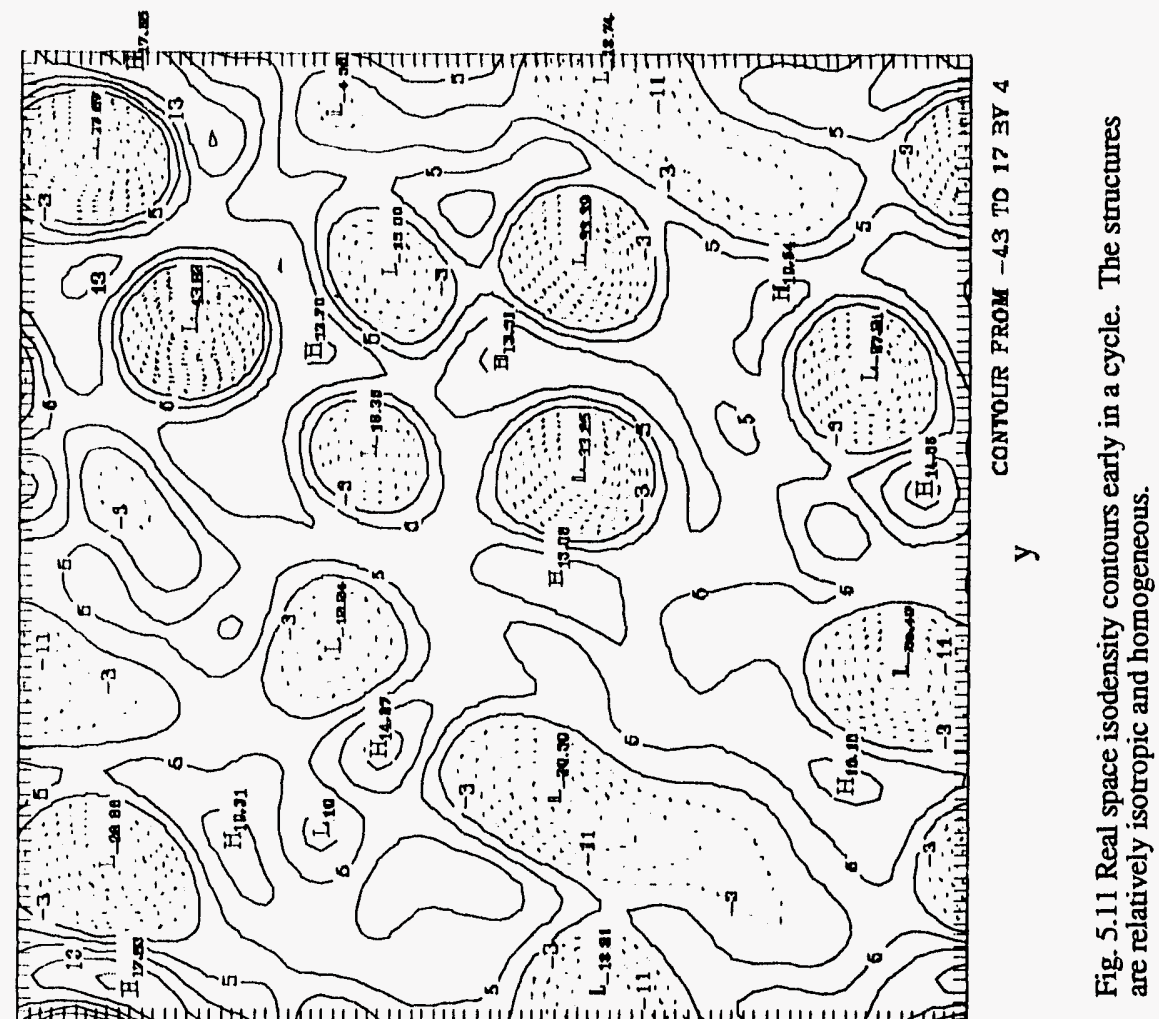


$\stackrel{m}{9}$
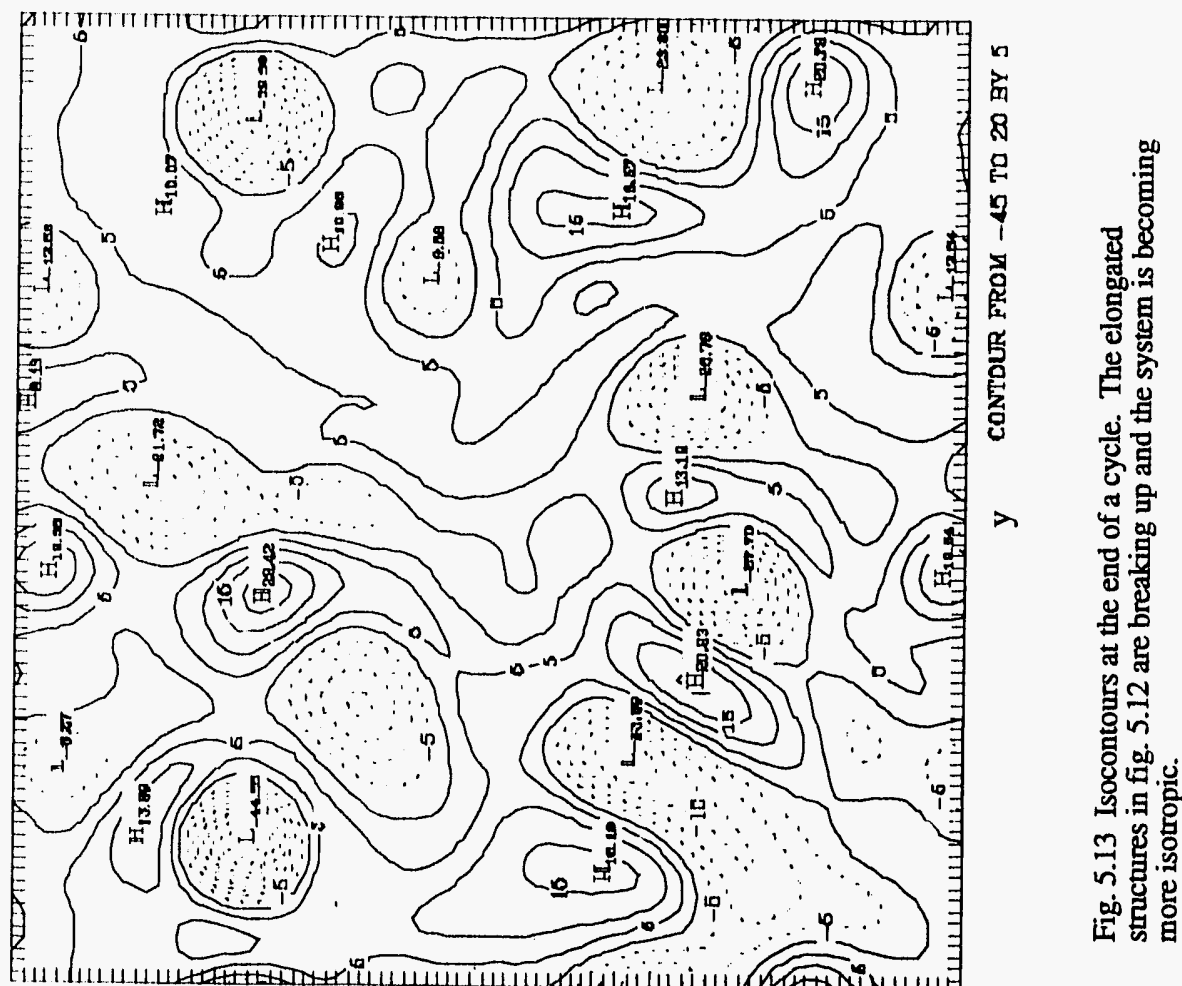


\subsection{Conclusions}

Using the same variety of diagnostics as employed for the one-field model, including transfer rates, wavenumber and frequency spectra, mode, spectrum and flux histories, the dynamics of a two-field model has been investigated. It is found that the ExB and polarization drift nonlinearities behave as they would alone in the region of $\mathrm{k}$ space in which they dominate. Both the nonlocal anisotropic direct cascade of the $\mathrm{E} \times \mathrm{B}$ nonlinearity and the local, isotropic, dual cascade of the polarization drift nonlinearity are observed. The two-field model dynamics reduces to the one-field dynamics in the high veff limit as expected. Most significantly, large scale fluctuations (cycling) are found to spontaneously occur in cases which include the cross over region in the system. This cycling is observed on a $-10 \tau_{\mathrm{e}}$ time scale with oscillation levels of $15-80 \%$ of the base saturation level. The existence of these fluctuations calls into question the validity of the assumption that saturated turbulence is also stationary or steady state. Fluctuations are observed in both the frequency and $\mathbf{k}$ spectrum coincident with the global fluctuations. These observations combined with the fact that bursting of transfer and flux are also coincident with the cycles suggests a relaxation type oscillation which is not inconsistent with a heuristic model that was outlined.

Characteristics observable in real space include structures elongated in the $\mathrm{x}$ (radial) direction. These structures propagate poloidally and break up, then reform on a cycle time scale. It is important to note that if an observer is sampling across multiples cycle the cycles may be washed out giving rise to an apparently broadened spectrum, but missing much of the important dynamics.

\section{References}

1. Y.-M. Liang, P.H. Diamond, X.H. Wang, D.E. Newman, and P.W. Terry, “A Two-Nonlinearity Model of Dissipative Drift Wave Turbuience", Phys. Fluids B, submitted.

2 G.G. Craddock et al, Transport Task Force Meeting Newport RI 1993 


\section{Chapter 6}

\section{Conclusions}

Throughout the study of turbulence there are a number of generally accepted principles upon which much of turbulence theory has been built. These basic precepts include the idea of a classic inertial range, isotropy of systems without anisotropic driving, conservative cascades, stationarity of saturated systems and a rough additive principle of nonlinearities. While these ideas largely work very well in the systems for which they were developed, the question of their broader use arises.

Using a series of simple models with extensive diagnostics designed for investigating the nonlinear dynamics, those basic assumptions are investigated in more complex systems then the "simple" Navier-Stokes system in which they were originaly developed. The models used have been, in order of increasing complexity,as follows. A one-field model for Dissipative Trapped Ion Convective Cell (DTICC) turbulence which has one nonlinearity $(\mathrm{E} \times \mathrm{B})$ and is used to study the dynamics of an anisotropic nonlinearity. A one-field model for Hasagawa - Mima (H-M) turbulence which also has one nonlinearity (polarization drift) and is used to study dual cascade dynamics. A one-field model of Dissipative Trapped Electron mode (DTEM1) turbulence with both the ExB and the polarization drift nonlinearities which lends itself to the investigation of the interplay between the nonlinearities. And finally a two-field two-nonlinearity model of dissipative trapped electron model turbulence (DTEM2) is used to investigate feedback of two nonlinear effects and nonstationarity. In all of these models the diagnostics used stress the transfer dynamics and included time histories of total energy and enstrophy as well as time histories for individual modes. These are supplemented with direct transfer diagnostics in addition to frequency and $\mathrm{k}$ spectra evolution. With these tools it is possible to investigate the detailed dynamics of the mode evolution and spectral transfer.

The basis for the Kolmogorov spectrum is the assumption that inertial ranges are dominated by a lecal self-similar transfer or cascade. Using the Kolmogorov spectrum and equilibrium statistical mechanics it is possible to infer the preferred direction of flow for a given nonlinearity. The dynamics of most turbulent systems are governed by nonlinearities which are themselves isotropic so that in the absence of anisotropic driving, damping or geometry the turbulence is also expected to be isotropic. The DTICC model is used to study the dynamics of an anisotropic, nonstandard nonlinearity. It is found that this nonlinearity transfers energy nonlocally from low ky to high ky. This leads to a saturated spectrum much flatter in the ky direction than that predicied by Kolmogorov theory. This should not be surprising as the lack of a local transfer induced similarity range precludes the use of Kolmogorov theory.

Going hand in hand with the concept of local transfer in an inertial range is the idea of conservative cascades. The H-M model is used to study the dynamics of the polarization drift nonlinearity $\left(\nabla \phi \times z \cdot \nabla \nabla^{2} \phi\right)$ which has two quadratic invariants, energy and enstrophy. These two invariants give rise to the same dual cascade that is familiar from 2-D Navier-Stokes turbulence. In this cascade energy is transferred conservatively to low $\mathbf{k}$ and enstrophy is transferred conservatively to high $\mathbf{k}$. The conventional description of the dual cascade was formulated by Kraichnan who used a spectrum of infinite extent to show that energy alone is cascaded in the low $\mathbf{k}$ region and enstrophy alone is cascaded in the high $\mathbf{k}$ region. It is found here that if the spectrum is not infinite in extent there is some "improper' transfer. That is, in the energy transfer range some enstrophy is transferred and in the enstrophy transfer range some energy is transferred. The amount of "improper" transfer depends on both the extent of the inertial range and the position within that range of the source of free energy (the injection range). It is quite possible to arrange a situation in 
which more energy or enstrophy flows in the improper direction then in the proper direction. This is imporant to keep in mind when analyzing system dynamics from the conservative cascade point of view.

Nonlinearities by definition cannot be linearly superimposed as if they were independent, yet it is assumed that if two nonlinearities have different regions of dominance in $\mathrm{k}$ space, in the region dominated by one nonlinearity the other nonlinearity can be ignored. The DTEM1 model was used to investigate the effects of two nonlinearities interacting in one system. It was found that at the extremes in which one dominates the other by more then an order of magnitude it is justifiable to drop the sub-dominant nonlinearity. However, in the often large region where the nonlinearities relative strength are within an order of magnitude of each other, new dynamics occur which are not explainable by a linear super-position of the two nonlinearities. The "cross-coupling" dynamics are qualitatively different and include both a change in the transfer dynamics and a nonlinear frequency shift. The frequency shift can be as large as a few times $\omega^{*}$ and is found to be proportional to $\mathrm{k}_{\mathrm{y}}$. The shift observed from simulation qualitatively agrees with the shift predicted by closure theory. Such shifts could help explain experimentally observed frequencies which seem anomalously shifted away from the frequency expected from linear dispersion relations. The influence of the crossover region creates five effective regimes: 1 ) at the low $\mathrm{k}$ extreme transfer dynamics are governed solely by the ExB nonlinearity; 2) at $\mathrm{k}$ values between the crossover region and the region of $E \times B$ dominance both the $\mathrm{E} \times \mathrm{B}$ and the cross-coupling dynamics are important; 3 ) in the crossover region, the characteristic dynamics are dominated by the interaction of the two nonlinearities and these dynamics are largely independent of the dynamics of the $\mathrm{E} \times \mathrm{B}$ and polarization drift nonlinearities when acting alone; 4) in the region above the crossover region but below the polarization drift dominated region, both the interaction of the noniinearities and the polarization drift dynamics are important; and 5) at the highest $k$ values the polarization drift dynamics dominate. Before ignoring a subdominant nonlinearity it may be imporant to assure oneself that the nonlinearity of interest is sufficienty dominant so as to dominate both the cross-coupling dynamics as well as the subdominant nonlinearity.

In order to self consistently include the feedback effect of the frequency shift on the linear growth and particle flux, it is necessary to extend the model beyond a one-field model to a two-field model. The DTEM2 model is used to study these interaction feedbacks. The most striking result from the two-field studies is the lack of a stationary saturated state. As with most driven damped turbulent systems, this system does reach a saturated state dependent on the various parameters. These parameters include growth, damping and the ratio of ExB to polarization drift nonlinearity strength but, in the case of fully developed turbulence, do not include initial conditions. In this system the saturated state is found to be non-stationary, exhibiting oscillations (cycles) with amplitude of order $50 \%$ and oscillation period on the order of 10 eddy turnover times. During these cycles most of the relevant quantities also undergo large fluctuations. These include flux and transfer rates as well as frequency and $\mathrm{k}$ spectra. These cycles may be understood in terms of a simple heuristic relaxation oscillation model. It is possible that this type of oscillation may in fact exist in experiment, but is effectively "washed out" by diagnostics that average over a few cycles. Since these models are all local, it is also possible that spatial averaging could wash out the fluctuations. If they do exist it might suggest that transport is a much more locally intermittent event then generally believed.

In conclusion, an outgrowth of this thesis is that there are three areas in which general turbulence lore may not be valid. There do exist nonlinearities for which transfer is not local in $\mathrm{k}$ space (ExB nonlinearity) in disagreement with Kolmogorov scaling. It is not always justified to discard suhdominant nonlinearities because of the fundamentally new dynamics that can arise from cross-coupling of multiple nonlinearities. And finally, saturated states are not always stationary and can infact exhibit complex cycling with 
ramifications for many observable quantities. It is important when dealing with turbulent nonlinearities to be cognizant of these large variety of dynamical behaviors that can be displayed. One should not get complacent in the process of extrapolating from a well known system to an unknown one.

\section{APPENDIX A}

\section{Computational Model descriptions}

The computational results in this thesis are really a compilation of results from four codes of two different types. The first code was a one-field spectral code with one nonlinearity (EXB). This code used an MMSL routines (DB2QBF) to do the time stepping When the second nonlinearity was added the code was changed to a more portable solver of the same type called LSODE by Alan C. Hindmarsh at LLNL. At that time the Jacobian of the system was explicitly entered and the flow diagnostics where added The next code was a pseudo-spectral version of the one-field two nonlinearity code. This code was made in order to increase the $\mathbf{k}$ space sizes which were practical to run (as pseudo-spectral is more efficient) as well as to serve as a check on the validity of the spectral results. Finally, the two-field two nonlinearity code, as a spectral code. Spectral codes were used because thei accuracy is limited only by the specified precision and the number of modes in the truncation. They work by Fourier transforming (in space) the PDE and truncating the Fourier representation at some value of $\mathbf{k}$ This process is exact for most PDE's, if $\mathbf{k}$ is taken to go to infinity, which is clearly not possible. However, in most systems there is some physical mechanism which realistically cuts off the $k$. This can be as simple as kinematic viscosity (or hyperviscosity) or it could be some complicated mechanism by which the model of interest loses validity at high $\mathbf{k}$ and must couple to new modes which may be parameterized as a damping or driving at high $\mathbf{k}$. We have typically used both methods combined into one by including viscosity or hyperviscosity as a high $k$ cutoff to simulate either real viscosity or an energy sink into coupling with some new higher $k$ mode. The main drawback of pure spectral codes is that nonlinearities become a convolution in $\mathbf{k}$ 
space, i.e.

$\sum_{\mathbf{k}^{\prime}} \mathbf{k} \times \mathbf{k}^{\prime} \cdot \mathbf{z}\left[\left(k_{\perp}-k_{\perp}\right)^{2}-k_{\perp}{ }^{2}\right] \phi_{\mathbf{k}^{\prime}} \phi_{\mathbf{k}-\mathbf{k}^{\prime}}$, for each mode.

Clearly, doing $k_{n}{ }^{2}$ computations is much less efficient than doing $k_{n} \ln k_{n}$. Therefore, the pseudo-spectral method was developed. Pseudo-spectral codes have exactly the same accuracy as spectral codes, as long as the $\mathrm{k}$ space they are operating in is $50 \%$ larger. The idea behind pseudo spectral codes is that new Fast Fourier Transform methods are efficient enough to made it advantageous to move back and forth between real space and $k$ space with the products (nonlinear terms) being evaluated in real space and the time-advancing (as well as the linear terms) being computed in $\mathrm{k}$ space. The computational requirements for the spectral codes versus pseudo spectral codes goes as $n^{2}$ versus $n$ in $n$ where $n$ is the total number of modes simulated. This difference approaches a very significant level rather rapidly. Unfortunately, since we are interested in the flow in $\mathbf{k}$ space which intrinsically requires calculation of the convolution, we are generally unable to benefit from pseudo spectral codes except as a check of the spectral method.

The systems explored here are extremely stiff systems. This means physically that there are many different time scales simultaneously important in the problem. Mathematically this implies that the Jacobian is sparse. Computationally this means that most simpler time (integrating) methods such as Runga-Kutta do not work well. Even simple predictor-corrector routines had trouble converging hence, the method used was one specifically written for stiff systems. The method employed is the Gear method in the LSODE solver package. This package is an adaptive time stepping predictor-corrector implementation of the Gear solver and is available to the public from netlib. With this routine one is able to obtain results with a fixed time step while allowing the solver to use a variable time step. This is important for some post processing such as frequency analysis. Typically we try to choose a time step that gave approximately 10 points per eddy turnover time outputted which normally gives approximately 10 computational time steps per outputted time step.

The code itself is portable enough to run on any machine with ANSI compatible FORTRAN 77 or FORTRAN 90 and has been run on everything from a Macintosh II to a Cray C-90. Most of the computation was performed on a DEC station 5000-240, a Cray-2, a Cray-YMP, and a Cray C-90 with some work done on a DEC station 3000-400 AXP (an alpha box). The largest spectral simulations (51x51) were performed on the C-90 and took up to ten days of CPU time. Most of the FORTRAN post processing such as the frequency spectra analysis routines are run on the DEC station 5000 . The fmal post processing (the imaging) is performed mainly on Apple Macintosh computers. The time plots are made with Kalidagraph, the 3-D plots are made with Wingz, and the movies are made with NCSA Image. The real space contour plots are created on the DEC station using NCAR graphics.

\section{Below is a listing of the spectral code for the 2 field runs}

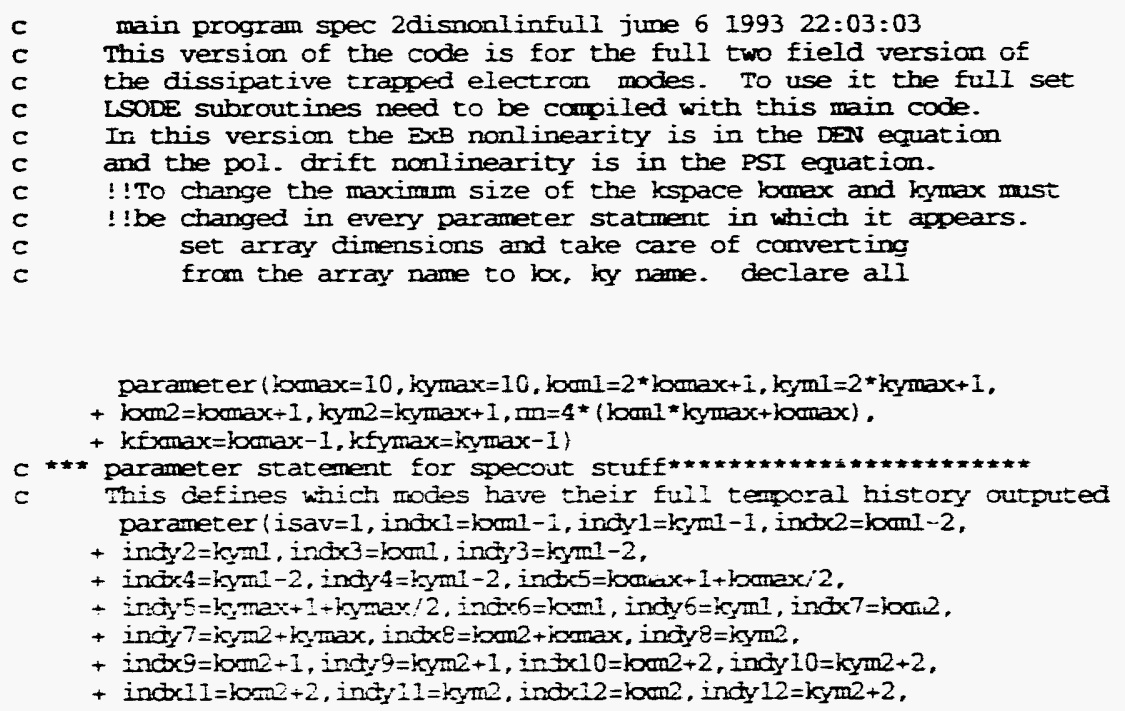


+ indx $13=\mathrm{komax}+1+\mathrm{kxmax} / 2$, indy $13=\mathrm{kym} 2$, indx $14=\mathrm{bcn} 2$,

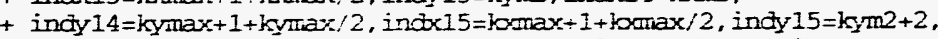



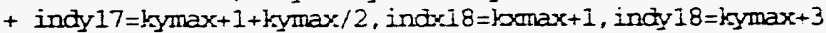

+ ind $19=$ komax +1 , ind $19=k_{y} m a x+4$, ind $20=$ bomax +1 , indy $20=k y$ max +5 ,

+ indx $21=$ komax +1 , indy $21=k y \max +6$, incic $22=\mathrm{komax}+1$, indy $22=\mathrm{kymax}+7$

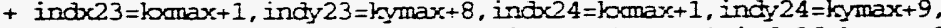

+ indx $25=\mathrm{k}$ max +1 , indy $25=\mathrm{kgmax}+10$, indx $25=\mathrm{komax}+1$, indy $26=\mathrm{kymax}+11$

+ ind $27=\mathrm{k} x \mathrm{max}+1$, indy $27=\mathrm{kymax}+12$, indr $28=\mathrm{komax}+1$, indy $28=\mathrm{kymax}+13$

+ indr $29=\mathrm{kxmax}+1$, indy $29=\mathrm{kgmax}+14$, indx $30=\mathrm{k} \times \mathrm{max}+1$, indy $30=\mathrm{kymax}+15$

+ indx $31=k x m a x+1$, indy $31=k y m a x+16, i n d x 32=k m_{m a x}+1$, indy $32=k y m a x+17$,

+ indx $33=k x m a x+1,1$ ndy $33=k y m a x+13$, indx $34=b x \max +1$, indy $34=k y m a x+19$

+ indx $35=\mathrm{komax}+1$, indy $35=\mathrm{kymax}+20$, indy $36=\mathrm{kymax}+1$, indx $36=\mathrm{komax}+3$

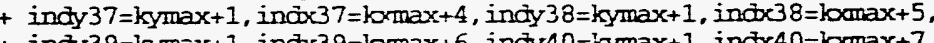

+ indy $39=k_{r} \max +1$, indx $39=\mathrm{komax}+6$, indy $40=\mathrm{kymax}+1$, indx $40=\mathrm{komax}+7$

+ indy $41=$ kymax +1 . ind $41=$ lamax +8 , indy $42=$ kymax +1 , indx $42=\mathrm{kamax}+9$,

+ indy $43=\mathrm{kymax}+1$, ind $43=\mathrm{kxmax}+10$, indy $44=\mathrm{ky}$ ax $x+1, \operatorname{indx} 44=\mathrm{kxmax}+11$

+ ind 4 (n

+ indy $17=\mathrm{kmax}+1$, ind

+



c The complex variable declarations

complex psi (koml , kyml) , der (koml, kymi) , flowpx (kfomax) .

+ flowpy (kfymax)

+ flowpxl (kí.max), flowpxn (kfxmax), flowpyl (kfymax), flowpyn (kfymax),

+ flowexl (kfxmax), flowexn (kfxmax), floweyl (kfymax). floweyn (kfymax).

+ flowex (kfxmax), flowey (kfymax), enflowx (kfomax), enflowy (kfymax).

+ emnflwy (kfymax), enlflwy (kfymax), ennflwx (kfmax), enlflwx (kfxmax)

+ eneflowx (kfomax), eneflowy (kfymax), enneflwy (kfymax)

+ enleflwy (kfymax), enneflwx (kfxmax), enleflwx (kfxmax)

complex spec1 (30000), spec2 (30000), spec 3 (30000), spec4 (30000:

$+\operatorname{spec} 5(30000), \operatorname{spec} 6(30000), \operatorname{spec} 7(30000), \operatorname{spec} 8(30000), \operatorname{spec} 9(30000)$

$+\operatorname{spec} 10(30000), \operatorname{spec} 11(30000), \operatorname{spec} 12(30000), \operatorname{spec13}(30000)$.

+ spec14 (36,00), spec15 (30000), spec16(30000), spec17(30000).

+spec18 (30000), spec19(30000), spec 20(30000), spec 21 (30000).

+spec22 (30000), spec23(30000), spec24 (30000), spec25 (30000).

$+\operatorname{spec} 26(30000), \operatorname{spec} 27(30000), \operatorname{spec} 28(30000), \operatorname{spec} 29(30000)$.

+spec 31 (30000), spec 32 (30000), spec 33 (3000), spec 34 (30000):

+ spec $35(30000), \operatorname{spec} 36(30000), \operatorname{spec} 37(3000)), \operatorname{spec} 38(30000)$.

+ Spec $39(30000), \operatorname{spec} 30(30000)$, spec40 (30000), spec41 (30000),

+spec42 (30000), spec43 (30000), spec44 (30000), spec45(30000).

+spec50 (30000), spec51 (30000), spec52 (30000), spec53 (30000)

The real variable declarations

real konorm, kynorm, ekkkold ( $k x m l$, kyml), le, Ip, leout, lemid, lpout,

+ inmid, ratio2, edenold (boml, kyml), nue, emass, dfirst, nhos

The 3 common block declarations

cormon /parms/

+ v1, gamal, gama2, epsi, le, lp, bog, kyg, kmorm, kynom, bxs, kys,
+ nwIt, nwrs, $t$, ct, tol, ek, gamk, Elux, ncl, ratio,psio, d, anid, dout, loc, + init, flreal, flima, ejkcld, enirold, flexold, flowex, flowey, gama3.

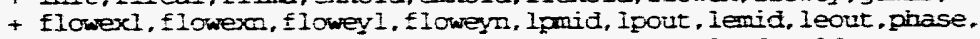

+ flpxold, flowpx, fiowpy, flowpxl, flowpxn, flowpy 1, edenold, hue.

+ amp, flowpyn, en, gamen, ed, et, ekcid, enold, enflowx, enflowy, enass.

t enneflwy, enleflwy, enneflwox, enlef Iwx. puithgt, locpul, kypui.

+ ratio2, joreak

common /arrays

+ psi,den

common farrdim

nt, ntstep, pesu

integer to(15), ti(15), timer(15)

dimension the plot variables and then open all the

unit 1 which

is the cray unit for the screen

real time $(10000)$,plot $1(10000), p l o t 2(10000), \operatorname{plot} 3(10000)$ call link ("unit $1=($ terminal $) / / ")$

pen (4, file = 'nonindat' , status = ' unimown'

pen (10, file=' nonoutdat', status=' 'uninown', recl=500)

pen (7, file ' 'nondat', status=' unimown' , recl $=5000$ )

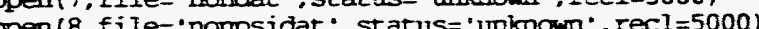

apen (9. file=' nonsrordat' , status =' unlnown')

gpen (11, file= 'nonspecp' , status = ' unkown' , recl $=15000$ )

"

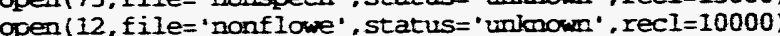

open (13, file $=$ ' noniflowe' , status $=$ ' monomom' ', recl $=10000$ )

open (14, file= ' nonspkek' ', status = 'unknown' )

open (15, fil $=$ ' nonflowp' , status = ' unionown' , recl $=10000$ )

open (16, file ='noniflowp' , status $={ }^{\prime}$ unknown ${ }^{\prime}$, recl $I=10000$ )

open (17, file = ' nonensdat' ', status = ' unknown' , recl $=5000$ open (18, file=' 'nomiat ', status=' 'unlonown', recl $=5000$ ) open (19, file = ' nondendat' , status = ' minnown' , recl $=5000$

open (21, file = ' specout 1' , status= ' 'mknown' open (22, fille=' spezout 2 ' ' status = ' minown'

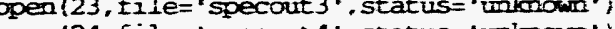
open (24. file = 'specout 4', status = 'unknown') cpen (25, file= 'specouts ', status= 'mknown'

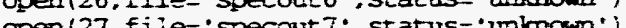

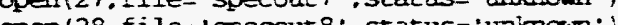



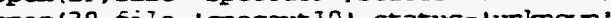
pen (31, file='specout 11. 'staturs='

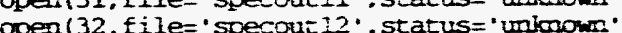
peen (33, file='specout 13 ' status=' 'uninom' '

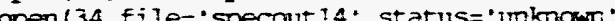
open (35. file=' specout 15." status=' unimown.

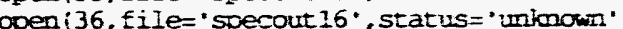
pen (37, file=' ' open $\{38$, file=' specout 18 ', status=' uninown') 
open (39, file= ' specout 19' , stat us= ' unknown' ')

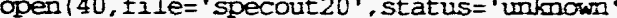
open (41, file $=$ ' specout 21 ', status= 'unknown' ' open (42, file $=$ ' specout 22 ', status= 'unionown' open (43, Iile= ' specout 23 ', 'status = 'unknown' ' open (44, file= ' specout $24^{\prime}$ ', status = " mnknown') open (45. file = ' specout $25^{\prime}$ ', status = ' unknown' open (46, file = ' specout 26 ' , status = ' unknown' open (47, fil le = ' specout 27 ' ' status = ' unknown' open (48, file= ' specout 28 ', status = ' mbnown' ' open (49, file = 'specout 29 ', status = ' unknown' open (50, file $=$ ' specout $30^{\circ}$ ', status = ' unknown' open (51, file =' specout 31 ', status= 'unknown' open (52, file $=$ ' specout 32 ', status = 'unknown"

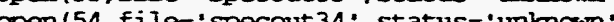

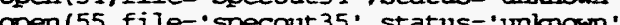
'pen (56, file ' 'specout $36^{\circ}$ ' status=' 'unhom' gpen (57, file=' specout 37' status=' 'umboom') open (58, file=' specout 38 ' 'status=' ' untom.' open (59, file=' specout 39 ', status = ' unionom.' open $\left(60\right.$, file $=$ ' specout $40^{\circ}$, status $=$ ' unimown' open (61, file=' specout 41 ', , status = ' unknown' open (62, file $=$ ' specout 42' ', status = ' unknown' " open $\left(63\right.$, fille $=$ ' specout $43^{\circ}$, status $=$ ' unionown' open (64, file= ' specout 44 ", status = 'unkonown' ' open (65, file= ' specout 45', status=' unkonown') open (66, file $=$ ' specout $46^{\prime}$, status $=$ ' unknown' open (67, fille=' 'specout 47', status = 'unkonown' ' open (68, file= 'specout 48', status = 'unknown' ') open $(69$, file = 'specout 49 ' ' status = ' unknown' ' open (70, file =' speccout 50' , status = 'unknown' open (71, file $=$ ' specout 51 ', status = 'unknown'

open (72, file= 'specout52' ', status = 'unkmown' open 773 , file 'specout5"',

"

open (20, file='restart')

c initialize

ierr=izchgt in (to)

c The subroutine initial sets up and initializes the various arrays and reads in the values of the setable parameters

call initial

This is the begiring of the main loop in which the timesteping occurs. Ffter the actual time stepping routine is called (in timead) the various dianstic ard printing routines are called. They are called at varying intervals. Ine intervais are determined by the variables rwirt and nurs. c eime step
do 20 i=1, ntste
$n+i=i$
mid=ntstep $/ 2$

solve ode's doing the actual iime step integrations

\section{call timead}

't depencing on the value of isav thays at eact (or some) time step if (isav ne. 1) then

call writdat

elseifl(isay .eq. 1) .and. ( (ncl .eq. 0).or.

$+($ modincl.10*mart) .eq- 0)) then

call writda

c

call writinit

c write the output camplex timeseries for a selection of $k$ space modes

$\operatorname{spec} 1(i)=p s i($ indx 1, indy 1 )
$\operatorname{spec} 2(i)=p s i($ indx2, indy 2 )

spect (i)

specs (i) 0 si (indx 5 indy 4

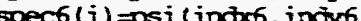

spec $7(i)=0$ si (inde 7 inty)

$\operatorname{spec} 8(i)=0 s i$ (indx8, indy)

spec9 (i) = rsi (indxo, indys)

spec10 (i) $=2$ si (indxio, ind 10

speclI (i) $=p$ si (inde11 indv11)

$\operatorname{spec} 12$ (i) $=$ psi (indk12, indr12)

$\operatorname{spec13}(i)=p s i$ (indbc13, indy 13)

spec14 (i) =psi (indel14, indy14)

spec15 (i)=Fsi (indoc15, indy 15)

spec16(i) =psi (indx16. indy 16)

spec17(i) =psi (indocl? indy 17)

spec18 (i) =psi (indel18, indy 18)

spec19 (i) =psi ( indris, indy 19)

spec20 $(i)=p s i$ (inde20, indy 20 )

spec21 (i) $=$ psi (indr21. indy 21)

$\operatorname{spec} 22(i)=p s i$ (indic22. indy 22)

spec 23 (i) =psi (indo23. indy 23)

spec 24 (i) =psi (indo 24, incy 24 )

spec 25 (i) =psi (ination. indy 25)

spec26 (i) =psi (indre26, inay 26)

spec28 (i) =psi (inating (indtind

pec28 (i) =

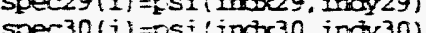

spec 31 (i) =psi(inot 31 ind 31 :

spec 32 (i) $=0 s i$ (ingta 32 ind 32 )

spec $33(i)=p s i$ (indr 33 . indt3 3 : 
$\operatorname{spec} 34(i)=p s i(i n d x 34$. indy 34$)$

spec 36 (i) $=$ psi (indx 36 . indy 36 )

spec37(i) $=$ psi (indx 37 , indy 37 )

spec 39 (i) $=0$ si (indx39. indy 38 )

spec 35 (i) =psi (intro35 indt35)

spec40 (i) $=$ psi $($ indx 40 , indy 40$)$

spec41 (i) $=p s i$ (indx41, indy 41 )

spec42 (i) =psi (indx 42 , indy 42 )

spec43 (i) $=$ psi (indx 43, indy 43)

spec44 (i) =psi (indre44, indy 44)

$\operatorname{spec} 45$ (i) $=$ psi (indx 45, indy45)

spec46 (i) $=$ psi (indx 46 , indy 46)

spec47(i) =psi (indx47, indy 47)

spec48 (i) $=$ psi (indx 48, indy 48)

spec49 (i) =psi (indx 49 , indy 49)

$\operatorname{spec} 50(i)=p s i$ (indx 50 . indy 50 )

spec $51(i)=p s i$ (indos 1 , indy 51 )

spec52(i)=psi (indx 52 , indy 52 )

$\operatorname{spec} 53(i)=$ psi (indx 53 , indy 53 )

$n l=i$

etal=d

mel=me

epsil=eepsi

ntstepl=ntstep

call spwrite (spec1, spec2, spec 3, spec4 , spec5, spec6.

+ spec7, spec8, spec9, spec10, spec 11 , spec 12 , spec13.

+ spec14, spec15, spec16, spec17, spec18, spec19, spec20.

+ spec 21 , spec 22 spec23, spec 24, spec 25 , spec 26 , spec27.

+ spec $28, \operatorname{spec} 29, \operatorname{spec} 30, \operatorname{spec} 31$. spec $32, \operatorname{spec} 33, \operatorname{spec} 34$.

+ spec 35, spec 36, spec 37, spec 38, spec 39, spec 40, spec41.

+ spec49, spec50, spec51, spec52, spec53,ni, etai, nuel, epsi1.

+ dt 1 , ntsteol

get energy's and mean sq flux, growth rates

call energy

every nwrt steps write to the plot arrays and to various spectum output arrays

if $(\bmod (n c 1,1$ witt $)$.eq. 0$)$ then

write to print diagnostics arrays

time(i) $=t$

$\operatorname{plot} 2(i)=e k$

write to spectum output arrays

call writeit call writesp

write the a selection of flux and psi**2 and den**2 arrays every

mars timesteps murs timesteps

write $\left(6,0^{*}\right)(\cdot i=\cdot, i\}$

\section{call writear}

call kflowp

if $((n c l$.eq. 1) or. (mod(ncl, mars) .eq. 0)) then

cali writksp

call storear

call writflwe

endif

20

cantimie

C 100 finish up

iff(ti(I3). le. 0) goto 100

200 o 200 i=1.13

overlap=real (timer (I) )/real (timer (13))

write $(6, *)$ (timer $(i), i=1,13$ )

write $(6, *)$ - overlape: , overlap

write $(10, *)$ itimer $(i), i=1,13$ )

write $(10, *)$ - overlap $=*$, overlap

call final

call ploc (time,plot1.plot2,plot3)

write $(6, *)$ "program terminated normally"

stop



subrout ine spwrite (spec1, spec2, spec3, spec4, spec5, spec6,

+ spec7, spec8, spec9, spec10, spec11, spec12, spec13.

+ spec14, spec15, spec16, spec17, spec18, spec19, spec20.

+ spec 21, spec 22, spec 23, spec 24. spec25, spec 26. spec27.

+ spec28, spec29. spec30, spec31, spec32, spec 33, spec34.

+ spec 35. spec 36, spec 37, spec 38, spec 39, spec40, spec41.

+ spec42, spec43, spec44, spec45. spec46. spec47, spec48.

+ spec49. spec50. spec 51, spec52, spec53.n. eta, me. epsi
+ di.ntstep)

c This subroutine saves the mode histories for a selected modes

c it also calculates the total growth rates for some modes 


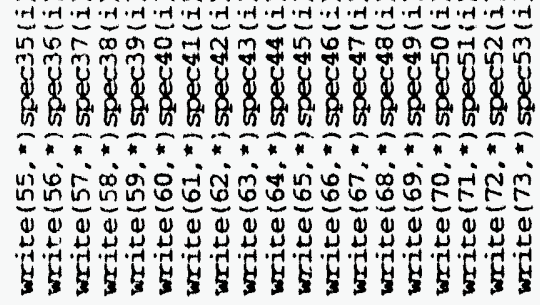

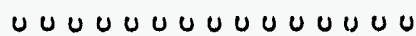

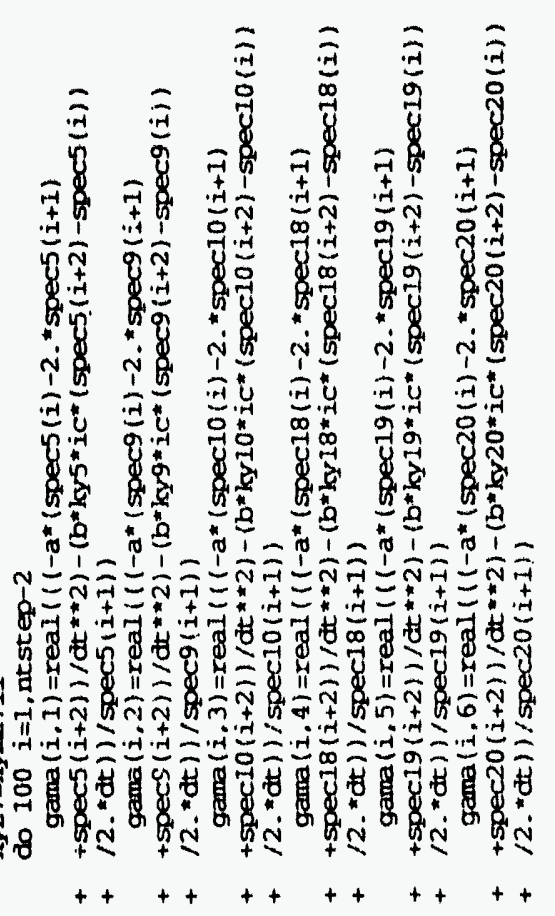

$\bar{n}$

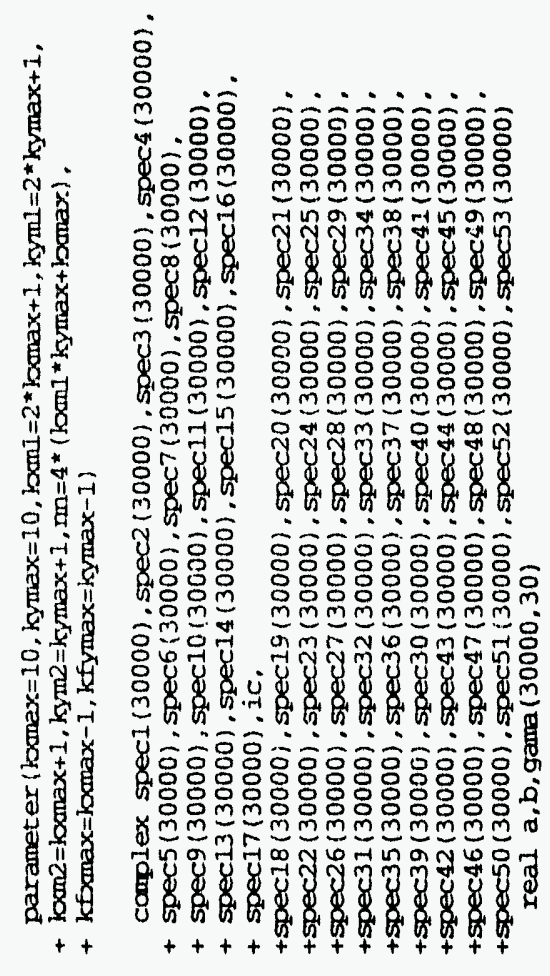

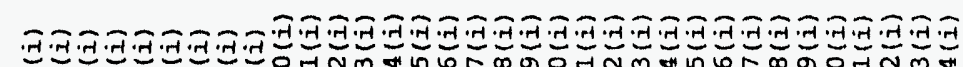
ชิษ

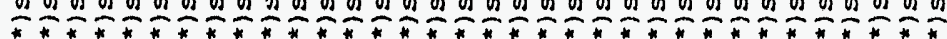
तुल O

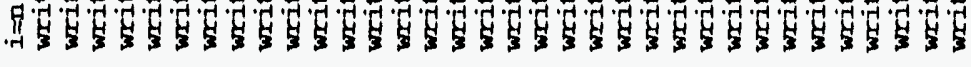


gama $(i, 7)=\operatorname{real}\left(\left(1-a^{\star}\left(\operatorname{spec} 21(i)-2 \cdot{ }^{*} \operatorname{spec} 21(i+1)\right.\right.\right.$

$\left.++\operatorname{spec} 21(i+2)) / a t^{\star \star} 2\right)-\left(b^{\star} k y 21^{\star} i c^{\star}(\operatorname{spec} 21(i+2)-\operatorname{spec} 21(i)\right.$ $d t) / \operatorname{spec} 21(i+1))$

gama $(i, 8)=$ real $\left(\left(<-a^{*}\left(\operatorname{spec} 22(i)-2 .{ }^{*} \operatorname{spec} 22(i+1)\right.\right.\right.$

$\left.++\operatorname{spec} 22(i+2)) / d t^{\star \star} 2\right)-\left(b^{\star} k y 22^{\star} i c^{\star}(\operatorname{spec} 22(i+2)-\operatorname{spec} 22(i))\right.$

$\left.\left.\left.12{ }^{*} \mathrm{dt}\right)\right) / \mathrm{spec} 22(i+1)\right)$

gama $(i, 9)=$ real $\left(\left(1-a^{*}(\operatorname{spec} 23(i)-2 *\right.\right.$ spec $23(i+1)$

$\left.++\operatorname{spec} 23(i+2)) / d t^{\star \star} 2\right)-\left(b^{\star} k y^{2} 3^{\star} i c^{\star}(\operatorname{spec} 23(i+2)-\operatorname{spec} 23(i))\right.$

$+12 . \star d t) / / \operatorname{spec} 23(i+1))$

gama $(i, 10)=\operatorname{real}\left(\left(\left(-a^{\star}\left(\operatorname{spec} 24(i)-2 .{ }^{*} \operatorname{spec} 24(i+1)\right.\right.\right.\right.$

$\left.+\operatorname{spec} 24(i+2)) / d t^{\star \star} 2\right)-\left(b^{\star} k y 24^{\star} i c^{\star}(\operatorname{spec} 24(i+2)-\operatorname{spec} 24(i))\right.$

$+12 . \star d t)(/ \operatorname{spec} 24(i+1))$

gama $(i, 11)=\operatorname{real}\left(\left(\left(-a^{*}(\operatorname{spec} 25(i)-2 . * \operatorname{spec} 25(i+1)\right.\right.\right.$

$\left.++\operatorname{spec} 25(i+2)) / d t^{\star \star} 2\right)-\left(b^{\star} k y 25^{\star} i c^{\star}(\operatorname{spec} 25(i+2)-\operatorname{spec} 25(i))\right.$

$+12 . * \mathrm{dt})(1 \mathrm{spec} 25(i+1))$

gama $(i, 12)=\operatorname{real}\left(\left(\left(-a^{*}(\operatorname{spec} 26(i)-2 . * \operatorname{spec} 26(i+1)\right.\right.\right.$

$\left.+\quad+\operatorname{spec} 26(i+2)) / d t^{\star \star} 2\right)-\left(b^{\star} k y 26^{\star} i c^{\star}(\operatorname{spec} 26(i+2)-\operatorname{spec} 26(i))\right.$

$+/ 2 . \star d t)) / \operatorname{spec} 26(i+1))$

gama $(i, 13)=$ real $\left(\left(-a^{*}\left(\operatorname{spec} 27(i)-2 .{ }^{*} \operatorname{spec} 27(i+1)\right.\right.\right.$

$\left.++\operatorname{spec} 27(i+2)) / d t^{\star \star} 2\right)-\left(b^{\star} k y 27 * i c^{\star}(\operatorname{spec} 27(i+2)-\operatorname{spec} 27(i))\right.$

$+12 . * \mathrm{dt}) 1 / \mathrm{spec} 27(i+1))$

do $110 \quad i=1$, ntstep-2

write $(74, *)$ (gama $(i, j), j=1,13)$

110

\section{continue}

return

end

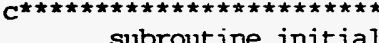

subroutier (knitial

$\left.+\mathrm{kxm} 2=\mathrm{k} \times \max +1, \mathrm{kym} 2=\mathrm{kymax}+1, \mathrm{nn}=4^{*}(\mathrm{kxm})^{\star} \mathrm{kymax}+\mathrm{kxmax}\right)$,

$+\mathrm{kfxmax}=$ komax $-1, \mathrm{kfymax}=\mathrm{k}(\mathrm{m} a \mathrm{x}-1)$

complex psi (kxml, kyml), den (koml, kyml), flowpx (kfxmax)

+ flowpy (kfymax)

+ flowpxl (kfxmax), flowpxn (kfxmax), flowpyl (kfymax), flowpyn (kfymax),

+ flowexl (kfxmax), flowexn (kfxmax), floweyl (kfymax), floweyn (kfymax),

+ flowex (kfxmax), flowey (kfymax), enf ] ‥vx(kfxmax), enflowy (kfymax),

+ ennflwy (kfymax), enlflwy (kfymax). inflwx (kfxmax), enlflwx (kfxmax),

+ eneflowx (kfxmax), eneflowy (kfymax), enneflwy (kfymax),
+ enleflwy (kfymax), enneflwx (kfxmax), enleflwx (kfxmax)

real kxnorm, kynorm, ekkold (koml, kyml), le, lp, leout, lemid, lpout,

+ Ipmid, ratio2, edenold (koml, kyml) , nue, emass, dfirst, rhos

cormon /parms/

+ v1, gama1, gama2, epsi, le, lp, kxg, kyg, kxnorm, kynorm, kxs, kys,

+ nwrt, nwrs, t, dt, tol, ek, gamk, flux, ncl, ratio, psio, d, dmid, dout, loc,

+ init,flreal, flima, ekkold, enkold, flexold, flowex, flowey, gama3,

+ flowexl, flowexn, flowey l, floweyn, I pmid, Ipout, lemid, leout, phase

+ flpxold, flowpx, flowpy, flowpxl, flowpxn, flowpyl, edenold, nue,

+ amp, flowpyn, en, gamen, ed, et, ekold, enold, enflowx, enflowy, emass,
+ ennflwy, enlflwy, ennflwx, enlf1wx, eneflowx, eneflowy, dfirst, rhos,

+ enneflwy, enleflwy, enneflwx, eriaflwx

common /arrays/

psi, den

comion /arrdim/

+ nt,ntstep, pesu

C

character ${ }^{2} 4$ fdate

external fdate

set quantities

ekold $=0$.

enold $=0$.

write $(10, *)$ fdate ()

read in parameters

call readit

initial perturbation

call pert

crite initial perturbation

write $(10, \star)$ " ncl $t$ ek en ed et flux phase gamk gamen"

write $(11, *) \cdot \operatorname{ekk}(0,0)$ ekk $(0,1)$ ekk(2) ekk(3) ekk(4) ekk(1,0)

$+\operatorname{ekk}(2)$ ekk(3) ekk(4) ekk (3,3).

write $(12,300) \quad(m, m=1, k f x m a x),(m, m=1, k f y m a x)$

write $(13,400)$ ( $m, m=1, k f x \max ),(m, m=1, k f y m a x)$

write $(15,301) \quad(m, m=1, k f x m a x)$, (m, $m=1, k f y \max )$

write $(16,401) \quad(m, m=1, k f x m a x),(m, m=1, k f y m a x)$

call writdat

call writeit

call writear

call writksp

300 format ( 3 ('flowerx', i2, 2x), 3('flowery', i2, 2x))

400 format ( 3 ('floweix', i2, 2x), 3('floweiy', i2, 2x))

301 format ( $(3$ ' 'flowprx', 12, $2 x), 3($ 'flowpry', $12,2 x))$

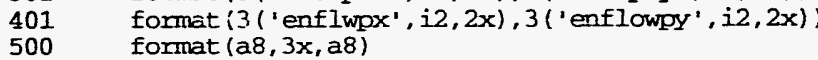
retur
end

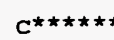

subroutine readit

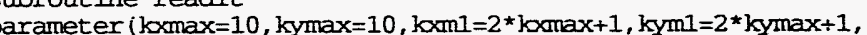

$+\mathrm{k} \times \mathrm{m} 2=\mathrm{k} \times \mathrm{max}+1, \mathrm{kym} 2=\mathrm{kymax}+1, \mathrm{nn}=4^{*}(\mathrm{k} \times \mathrm{m} 1 * k y \max +\mathrm{k} \times \max )$

+ kfxmax $=$ kxmax -1 , kfymax $=k y$ max -1 )

complex psi (kxml, kyml), den (kxml, kyml), flowpx (kfxmax).

flowpy (kfmax), flowpxn (kfxmax), flowpyl (kfymax), flowpyn (kfymax). 
+ flowexl (kfxmax), flowexn (kfxmax), floweyl (kfymax), floweyn (kfymax) + flo' ex (kfxmax), flowey (kfymax), enflowx (kfxmax), enflowy (kfymax), + ennilwy (kfymax), enlflwy (kfymax), ennflwx (kfxmax), enlflwx (kfxmax) + eneflowx (kfxmax), eneflowy (kfymax), enneflwy (kfymax)

real kxnorm, kynorm, ekkold (kxml, kyml), le, lp, leout, lemid, lpout,

+ lpmid, rat io2, edenold (kxml, kyml), nue, emass, dfirst, rhos

comon / parms/

+ v1, gama1, gama2, epsi, le, Ip, kxg, kyg, konorm, kynorm, koss, kys,

+ nwrt, nwrs, $t$, dt, tol, ek, gamk, flux, ncl, ratio, psio, d, ämid, dout, loc

+ nwrt, nwrs, $t, d t$, tol, ek, gank, flux, ncl, rat io, psio, d, amid, dout,
+ init, flreal, flima, ekkold, enkold, flexold, flowex, flowey, gama 3 .

+ flowexl, flowexn, floweyl, floweyn, lpmid, lpout, lemid, leout, phase,

+ flpxold, flowpx, flowpy, flowpxl, flowpxn, flowpyl, edezold, nue,

+ amp, flowpyn, en, gamen, ed, et, ekold, enold, enflowx, enflowy, emass,

+ enneflwy, enleflwy, enneflwx, enleflwx, pulhgt, kxpul, kypul,

+ ratio2, kbreak

cormon /arrays/

+ psi,den

cormon /arrdim/

+ nt, ntstep, pesu

choose whether you want to enter the data parameters or use the ones in the code

\section{integer ichoice}

write $(6, *)$ "type 2 for your file indat to enter the data,"

write $(6, *)$ "type 1 if you want to enter the data, type 0

write $(6, *)$ if you

read $(5, *)$ ichoice

if (ichoice eq. 0) then

input, from within code the various data parameters

data dt $/ 0.01 /$, ntstep $/ 100 /$, nwrt $/ 10 /$, nwrs $/ 100 /$, tol $/ 0.001 /$ data $\mathrm{v} 1 / 1.1$, gama1/1./,gama $2 / 1.1$, epsi/.001/, $\mathrm{kg} / 1 /$, kyg/1/

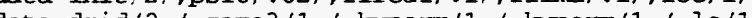

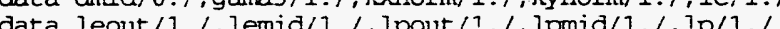

data pulhgt $/ 10 . /$, kxpul $/ 4 /$, kypul $/ 4 /$, kbreak $/ 4 /$, ratio $2 / 2$.

data emass 11.1 , dfirst 10.1 , rhos $/ .01 \%$

\section{elseif (ichoice .eq. 1) then}

or intere'ctively input the various data parameters

write $(6, *)$ "enter the values comma delimited"

write $(6, *)$ "dt, ntstep, nwrt, nwrs, tol, konorm, kynorm, loc" read $(5, *)$ dt, ntstep, nwrt, nwrs, tol, kxnorm, kynorm, loc write $(6, *)$ "v1, gamal, gama2, gama3, epsi, d, dout, kxg, kyg read $(5, *)$ v1, gamal, gama2, gama3, epsi, d, dout, kxg, kyg write $(6, *)$ "le, lemid, leout, lp, lpmid, lpout* read $(5, *)$ le, lemid, leout, lp, lpmid, lpou

write $\left(6,{ }^{*}\right)$ "amp(mult of tol to get atol), amid, kxs, kys

+ ratio (init slope), nue, emass, dfirst

read $(5, *)$ anq, amia, kxs, kys, ratio, nue, emass, dfirst write $(6, *)$ inic,psio, flreal, flina

read $(5, *)$ init, psio, flreal, flina

read $(5 *)$ pulhgt, kopul, kypul, kbreak, ratio2, rhos

else

or from input file (device 4 ) read $(4, *)$ dt, ntstep, nwrt, nwrs, tol, knorm, kynorm, loc read $(4, \cdot) \mathrm{vl}$, gamal, gama 2 , gama3, epsi, a, dout $\mathrm{kog}, \mathrm{kg}$ read $(4, *)$ le, lemid, leout, Ip, lpmid, lpout

read $(4, \star)$ amp, dmid, kxs, kys, ratio, nue, emass, dfirst read $(4, *)$ init, psio, flreal, flima

read $(4, *)$ pulhgt, loxpul, kypul, kbreak, ratio2, rhos

endif

namelist input

namelist / runp/ dt, ntstep, nwrt, Iwrs, tol, bonorm, kynorm, loc namelist /physp/ v1, gama1, gama2, gama3, epsi, d, dout, kxg, kyg namelist /intilp/ amp, dmid, kxs, kys, ratio, nue, emass, dfirst

namelist /pulse/ pulhgt, kxpul, kypul, kbreak, ratio2, rhos

write $\left(6,{ }^{\star}\right)$ 'xsize by ysize ', kxal, ' by ', kym

write $(6$, runp)

te $(6$, physp)

write $(6$, physp 2$)$

write $(6$, intilp)

write $(6$, pertin)

write $(10,)^{\prime}$ 'xsize by ysize ', kxml,' by ', kyml write (10, runp)

write (10, physp)

write $(10$, physp 2$)$

write $(10$, intilp)

write (10, pert in) namelist /physp2/ le, lemid, leout, lp, lpmid, lpout 


\section{subroutine endit (ifail, cout)}

called if there is an error in the intergrater see the integrater documentation for specific meaning

parameter $(\mathrm{kxmax}=10, \mathrm{kymax}=10, \mathrm{kxm1}=2 * \mathrm{k} \times \max +1, \mathrm{kynl}=2$ *kymax +1 ,

$+\mathrm{kxm2}=\mathrm{kxmax}+1, \mathrm{kym} 2=\mathrm{kymax}+1, \mathrm{nn}=4$ * $(\mathrm{kxm1}$ *kymax $+\mathrm{k} \times \mathrm{max})$,

$+\mathrm{kf}$ max $=\mathrm{kxmax}-1, \mathrm{kfy}$ max $=\mathrm{kymax}-1)$

complex psi (kxml, kyml), den (kxml, kyml) , flowpx (kfxmax)

+ flowpy (kfymax)

+ flowpxl (kfxmax), flowpxn (kfxmax), flowpyl (kfymax), flowpyn (kfymax)

+ flowexl (kfxmax), flowexn (kfxmax), floweyl (kfymax), floweyn (kfymax)

+ flowax (kfxmax), flowey (kfymax), enflowx (kfxmax), enflowy (kfymax),

+ ennf-wy (kfymax), enlflwy (kfymax), ennflwx (kfxmax), enlflwx (kfxmax)

+ eneflowx (kfxmax), eneflowy (kfymax), enneflwy (kfymax).

real kxnorm, kynorm, ekkold (kxml, kyml), le, lp, leout, lemid, lpout,

+ lpmid, ratio2, edenold

cormon /parms/

+ v1, gamal, gama2, epsi, le, lp, kxg, kyg , kxnorm, kynorm, kxs, kys,

+ nwrt, nwrs, $t, d t$, tol, ek, gamk, flux, ncl, ratio, psio, d, dnid, dout, loc,

+ init, flreal, flima, ekkold, enkold, flexold, flowex, flowey, gama3.

+ flowexl, flowexn, floweyl, floweyn, lpmid, Ipout, lemid, leout, phase

+ flpxold, flowpx, flowpy, flowpxl, flowpxn, flowpyl, edenold, nue,

+ amp, flowpyn, an, gamen, ed, et, ekold, enold, enflowx, enflowy, emass,

t ennflwy, enlelwy, enn liw, enlflwx, enefld

enneflwy, enlef

conion larrays/

psi,den

poming /arrdim

nt, ntstep, pesu
write $(10, \star)$ ifail, cout

write $(10, \star)$ ifail, cout

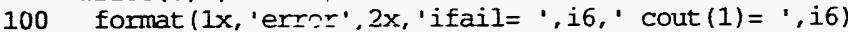

write $(6: *)$. Amm - orror termination

stop

end

subroutine writeit

parameter (kxma $10, \mathrm{kymax}=10, \mathrm{kxm} 1=2 \star \mathrm{kxmax}+1, \mathrm{kyml}=2 \star \mathrm{kymax}+1$

$+\mathrm{kxm} 2=\mathrm{kxmax}+1, \mathrm{kym} 2=\mathrm{kymax}+1, \mathrm{nn}=4$ * $(\mathrm{kxml}$ *kymax $+\mathrm{k} \times \mathrm{max})$,

$+\mathrm{kfxmax}=\mathrm{kxmax}-1, \mathrm{kfymax}=\mathrm{kymax}-1$

complex psi (kxml, kyml), den (kxml, kyml) , flowpx (kfxmax),

+ flowpy (kfymax)

+ flowpxl (kfxmax), flowpxn (kfxmax), flowpyl (kfymax), flowpyn (kfymax)

+ flowexl (kfxmax), flowexn (kfxmax), floweyl (kfymax), floweyn (kfymax)
+ flowex (kfxmax), flowey (kfymax), enflowx (kfxmax), enflowy (kfymax),

+ ennflwy (kfymax), enlflwy (kfymax), ennflwx (kfxmax), enlflwx (kfxmax)

+ eneflowx (kfxmax), eneflowy (kfymax), enneflwy (kfymax)

real kxnonm, kynorm, ekkold ( $k 0 m 1$, hym ), le, lp, leout, lemid, Ipout,

+ Ipmid, ratio2, edenold (kxml, kyml), nue, emass, dfirst, rhos

common /parms/

v1, gamal, gama2, epsi, le, lp, kxg, kyg, kxnorm, kynorm, kxs, kys.

nwrt, nwrs, $t$, dt, tol, ek, gamk, flux, ncl, ratio, psi0, d, dmid, dout, loc

+ init, flreal, flima, ekkold, emkold, flexold, flowex, flowey, gama3.

+ flowexl, flowexn, floweyl, floweyn, lpmid, lpout, lemid, leout, phase,

+ flpxold, flowpx, flowpy, flowpxl, flowpxn, flowpyl, edenold, nue,

+ amp, flowpyn, en, gamen, ed, et, ekold, enold, enflowx, enflowy, emass,

emnflwy, enlflwy, ennflwx, enlflwx, ene

common /arrays/

+ psi, den

cormon /arrdim/

+ nt, ntstep, pesu

write $(10,200) \mathrm{ncl}, t$, ek, en, ed, et, flux, phase, gamk, gamen

if(iscreen .ne. 1) then

write $(6,200) \mathrm{ncl}, t$, ek, en, ed, et, flux, phase, gamk, gamen

endif

200 format $(1 \mathrm{x}, \mathrm{i} 6,9(1 \mathrm{x}, \mathrm{e} 12.4)$

return

end

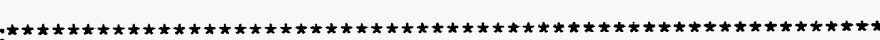

subroutine writesp

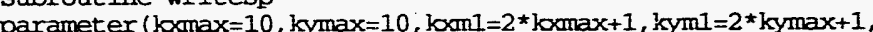

$+\operatorname{kxm} 2=k x \max +1, \mathrm{kym} 2=k y \max +1, \mathrm{nn}=4^{*}\left(\mathrm{kxm} 1^{*} k y \max +\mathrm{kxmax}\right)$,

$+\mathrm{kfx}$ max $=\mathrm{k}$ max $-1, \mathrm{kfymax}=\mathrm{kymax}-1)$

complex psi (kxmI, kyml), den (kxml, kymI), flowpx (kfxmax).

flowpy (kfymax)

+flowpxl (kfxmax), flowpxn (kfxmax), flowpyl ikfymax), flowpyn (kffmax).

+ flowexl (kfxmax), flowexn (kfxmax), floweyl (kfymax), floweyn (kfymax),

flowex (kfxmax), flowey (kfymax), enflowx (kfxmax), enflowy (kfymax),

ennflwy (kfymax), enlflwy (kfymax), ennflwx (kfxmax), enlflwx (kfxmax).

+ eneflowx (kfxmax), eneflowy (kfymax), enneflwy (kfymax),

real kxorm, kymorm, ekkold (kxml, kyml), le, lp, leout, lemid, lpout,

lpmid, ratio2, edenold ( $\mathrm{kxml}$, kyml), nue, emass, dfirst, rhos

common /parms/

+ vl, gamal, gama2, epsi, le, lp, kxg, kyg, kxnorm, kynorm, kxs, kys

+ nwrt, nwrs, t, dt, tol, ek, gank, flux, ncl, ratio, psio, d, dmid, dout, loc

+ init, flreal, flima, ekkold, enkold, flexold, flowex, flowey, gama3,

+ flowexl, flowexn, floweyl, floweyn, Ipmid, lpout, lemid, leout, phase,

+ flpxold, flowpx, flowpy, flowpxl, flowpxn, flowpyl, edenold, nue,

+ amp, flowpyn, en, gamen, ed, et, ekold, enold, enflowx, enflowy, emass,

+ ennflwy, enlflwy, ennflwx, enlflwx, eneflowx, eneflowy, dfirst, rhos,

+ enneflwy, enteflwy, enneflwx, enleflwx

common /arrays/

+ psi,den

cormon /arrdim/

+ nt, ntstep, pesu 
real ekk (kxml, kyml), edd ( $k \cdot 1, k v m l), \operatorname{nphi}(k x m l, k y m l)$

do 20 the er:ez:-

$\left.\mathrm{xk}=\mathrm{k} \times \mathrm{morm}^{*}: i-\mathrm{kxm} 2\right)$

$\mathrm{yk}=\mathrm{kynorm}^{*}(\mathrm{j}-\mathrm{kym} 2)$

ekk $(i, j)=0$ si $(i, j) * c$

ekk $(i, j)=p s i(i, j) * \operatorname{conjg}(p s i(i, j)) \star 2$.

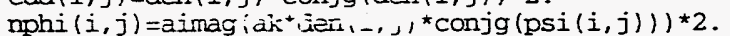

10

20 continue

write $(11, *) \quad(e k k(k x m 2, j), j=k y m 2+1, k y m 1),(e k k(i, k y m 2)$

$+\quad i=\operatorname{kom} 2+1, \operatorname{kam} 1), \operatorname{ekk}(\operatorname{kxm} 2+2, \operatorname{kym} 2+2), \operatorname{ekk}(\operatorname{kxm} 2+3, \operatorname{kym} 2+3)$

+ ekk (koml, kyml), (nphi (kom2,j),j=kym2+1, kyml),

$+(\operatorname{mphi}(i, \mathrm{kym} 2), i=\mathrm{kxm} 2+1, \mathrm{kxm})$

write $\left(75,{ }^{*}\right)$ (edd $\left.(\operatorname{kom} 2, j), j=\mathrm{kym} 2+1, \operatorname{kyml}\right)$, (edd ( $\left.i, \operatorname{kym} 2\right)$

$i=k \times m 2+1, k \times m 1)$, edd $(k x m 2+2, k y m 2+2)$, edd $(k x m 2+3, k y m 2+3)$

$+\operatorname{edd}(\mathrm{kxonl}, \mathrm{kym} 1)$

200 format $(50(e 13.6,1 x)$ )

return

end

C

subroutine writear

parameter $(\mathrm{kxmax}=10, \mathrm{kymax}=10, \mathrm{kxm} \mathrm{l}=2 \star \mathrm{kxmax}+1, \mathrm{kyml}=2 \star \mathrm{kymax}+1$,

$+\mathrm{kxm} 2=\mathrm{kxmax}+1, \mathrm{kym} 2=\mathrm{kymax}+1, \mathrm{nn}=4 *(\mathrm{kxm} 1 * \mathrm{kymax}+\mathrm{kxm} 2 \mathrm{x})$

kfxmax $=$ kxmax $-1, k f y \max =k y m a x-1)$

complex psi (kxml, kym1) , den (kxml, kyml), flowpx (kfxmax)

+ flowpy (kfymax)

flowpl (kfxmax), flowpxn (kfxmax), flowpyl (kfymax), flowpyn (kfymax)

flowexl (kfxmax), flowexn (kfxmax), floweyl (kfymax), floweyn (kfymax)

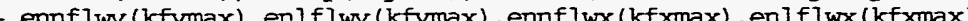

+ eneflowx (kfxmax), eneflowy (kfymax), enneflwy (kfymax),

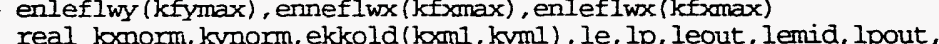

+ Ipmid, ratio2, edenold ( $\mathrm{kmm} 1$, kyml), nue, emass, dfirst, rhos

common /parms/

+ v1, gamal, gama2, epsi, le, lp, kxg, kyg, kxnorm, kymorm, kxs, kys,

+ nwrt, nwrs, $t$, dt, tol, ek, gamk, flux, ncl, ratio, psio, d, dmid, dout, loc,

+ init,flreal, flima, ekkold, emkold, flexold, flowex, flowey, gama3,

+ flowexl, flowexn, floweyl, floweyn, lpmid, lpout, lemid, leout, phase

+ flpxold, flowpx, flowpy, flowpxl, flowpxn, flowpyl, edenold, nue,

+ amo, flowpyn, en, gamen, ed, et, ekold, enold, enflowx, enflowy, emass,

+ ennflwy, enlf.wy, ennflwx, enlflwx, eneflowx, eneflowy, dfirst, rhos,

+ enneflwy, enleflwy, enneflwx, enleflwx

common /arrays/

+ psi,den

common /arrdirm/

+ nt, ntstep, pesu real ekk (kxml, kyml), eden ( $k a m l, k y m l)$

c calculate the energy in each mode

do $20 j=1$, kym

do $10 \quad i=1, k \times m$

$\mathrm{xk}=\mathrm{kxnOrm}^{\star}(\mathrm{i}-\mathrm{k} \times \mathrm{m} 2)$

$y k=k y n o r n^{*}(j-k y m 2)$

ekk $(2, j)=\operatorname{psi}(i, j){ }^{\star} \operatorname{conjg}(p s i(i, j)) \star 2$. +ekkold $(i, j)$

ekkoid $(i, j)=e k k(i, j)$

$j g(d e n(i, j)) \star 2 .+e d e n o l d(i, j)$

edenold $(i, j)=\operatorname{eden}(i, j)$

10 continue

20 continue

return

subroutine writdat

parameter ( kxmax $=10, \mathrm{kymax}=10, \mathrm{kxml}=2 \star \mathrm{k} m \mathrm{max}+1, \mathrm{kyml}=2 * \mathrm{kymax}+1$

$+k x m 2=k x m a x+1, k y m 2=k y m a x+1, n \pi=4 *$ ( $k x m 1 * k y m a x+k x m a x)$.

+ kfxmax $=$ kxmax -1, kfymax $=$ kymax 1 )

complex psi (kxml, kyml), den (kxml, kyml), flowpx (kfxmax),

+ flowpy (kfymax),

+ flowpxl (kfxmax), flowpxn (kfxmax), flowpyl (kfymax), flowpyn (kfymax)

+ flowexl (kfxmax), flowexn (kfxmax), flowey l (kfymax), floweyn (kfymax)

+ flowex (kfxmax), flowey (kfymax), enflowx (KSxmax), enflowy (Kfymax),

+ ennflwy (kfymax), enlflwy (kfymax), enmflwx (kfxmax), enlflwx (kfamax)

+ eneflowx (kfxmax), eneflowy (kfymax), enneflwy (kfymax),

enleflwy (kfymax), enneflwx (kfxax), enleflwy (kfmax)'

real konorm, kynorm, ekkold (kxml, kyml), le, Ip, leout, lemid, lpout,

lpmid, ratio2, edenold ( $\mathrm{kxml}$, kyml) , nue, emass, dfirst, rhos

common /parms

+ v1, gama1, gama2, epsi, le, lp, loxg, kyg, bonorm, kynorm, kxs, kys,

+ nwrt, nwrs, $t$, dt, tol, ek, gamk, flux, ncl, ratio, psio, d, dmid, dout, loc

+ init, freal, flima, ekkold, enkold, llexold, flowex, flowey, gama3,

+ flpxold, flowpx, flowpy, flowpxl, flowpxn, flowpyl, edenold, nue,

+ amo, flowpr, en gamen, ed, et, ekold, enold, enflowx, enflowy, emass,

+ ennflwy, enlflwy, ennflwx, enlflwx, eneflowx, eneflowy, dfirst, rhos,

+ enneflwy, enleflwy, enneflwx, enleflwx

common /arrays/

+ psi,den

common /arrdim/

do $20 j=1, k y m$

write $(7, *)$ (psi $(i, j), i=1, \operatorname{kxm} 1)$

$\operatorname{write}(18, *)(\operatorname{den}(i, j), i=1, \mathrm{kxm}$ )

write $(7, *)$

write $(18, \star)$ 
write $(18, *)$

return

end

subroutine writinit

use main

'restart')

do $20 i=1, \mathrm{kxm}$

c write $\left.\left(20,{ }^{\star}\right)(\operatorname{psi}(i, j), j=1, \mathrm{kym}]\right)$

c 20 continue

c close $(20)$

c return

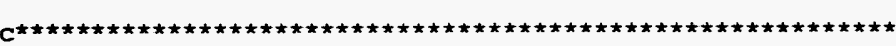

subroutine storear

parameter ( $k x \max =10, \mathrm{kymax}=10, \mathrm{kxml}=2 \star \mathrm{kxmax}+1, \mathrm{kyml}=2 \star \mathrm{k} k \mathrm{mmax}+1$,

$+\mathrm{kxm} 2=\mathrm{k} \times \mathrm{max}+1, \mathrm{kym} 2=\mathrm{kymax}+1, \mathrm{nn}=4 *(\mathrm{kxm} 1 * \mathrm{ky} \max +\mathrm{kxmax})$,

+ complex psi (kxml, kyml), den (kxml, kyml), flowpx (kfxmax),

+ flowpy (kfymax), flowpxn (kfxmax), flowpyl (kfymax), flowpyn (kfymax),

+ flowexl (kfxmax), flowexn (kfxmax), floweyl (kfymax), floweyn (kfymax)

+ flowex (kfxmax), illowey (kfymax), enflowx (kfxmax), enflowy (kfymax).

+ eneflowx (kfxmax), eneflowy (kfymax), enneflwy (kfymax),

+ enlefiwy (kfymax), enneflwx (kfxmax), enleflwx (kfxmax)

+ lpmid, ratio2, edenold (kxml, kyml), nue, emass, dfirst, rhos

common /parms/

+ v1, gama1, gama2, epsi, le, lp, kxg, kyg, knorm, kynorm, kxs, kys,

+ nwrt, nwrs, t, dt, tol, ek, gamk, flux, ncl, ratio,psio, d, dmid, dout, loc,

+ nwrt, nwrs, $t$, dt, tol, ek, gamk, flux, ncl, ratio, psio, d, dmid, dout, lo
+ init, flreal, flima, ekkold, emkold, flexold, flowex, flowey, gama3,

+ flowexl, flowexn, floweyl, floweyn, lpmid, Ipout, lemid, leout, phase,

+ flps:old, flowpx, flowpy, flowpxl, flowpxn, flowpyl, edenold, nue,

+ amp, flowpyn, en, gamen, ed, et, ekold, enold, enflowx, enflowy, emass,

+ +ennflwy, enlflwy, ennflwx, enlflwx, ene

cormon /arrays/

+ psi, den

common /arrdim/

+ nt, ntstep, pesu

rfal ekk ( $\mathrm{kxml}, \mathrm{kyml})$, ensk ( $\mathrm{kxml}, \mathrm{kyml})$, eden (kxml, kyml)

do $20 \mathrm{j}=1$, kyml

do $10 i=1, \mathrm{k} \times \mathrm{mm}^{2}$

$\mathrm{xk}=\mathrm{k} \times \mathrm{morm}{ }^{\star}(i-\mathrm{koxm} 2)$ $y k=k y n o r m *(j-k y m 2)$

if (ncl .le. 10) then

ekk $(i, j)=$ ekkold $(i, j)$

ensk $(i, j)=$ ekkold $(i, j) * a k$

eden $(i, j)=$ edenold $(i, j)$

else

ekk $(i, j)=e k k o l d(i, j) /$ real (nwrs)

ensk $(i, j)=$ ekkold $(i, j)$ *ak/real (nwrs)

eden $(i, j)=\operatorname{edenold}(i, j) /$ real (nwrs)

endif

elkold $(i, j)=0.0$

edenold $(i, j)=0.0$

10 continue

$c$ write the energies in array form

do $30 \mathrm{i}=\mathrm{k} \mathrm{xm} 2, \mathrm{k} \times \mathrm{mI}$

write $(8, *) \quad(e k k(i, j), j=1, k y m 1)$

write $(17, *)$ (ensk $(1, j), j=1, \mathrm{kyml})$

30 continue

write $(8, *)$

write $(8, *)$

write $(17, \star)$

write $(19, *)$

200 format (1050e13.5)

return

end

$C^{\star \star \star \star \star \star \star \star ~}$

parameter $($ kxmax $=10, k y m a x=10, k x m 1=2 \star k x m a x+1, k y m l=2 \star k y m a x+1$,

$\mathrm{kxm2}=\mathrm{kxmax}+1, \mathrm{kym} 2=\mathrm{kymax}+1, \mathrm{nn}=4 *(\mathrm{kxm1} * \mathrm{kymax}+\mathrm{kxmax})$

$+\mathrm{kfxmax}=\mathrm{kxmax}-1, \mathrm{kfymax}=\mathrm{kymax}-1)$

complex psi (kxml, kyml), den (kxml, kyml), flowpx (kfxmax) ,

+ flowpy (kfymax).

+ flowpxl (kfxmax), flowpxn (kfxmax), flowpyl (kfymax), flowpyn (kfymax),

+ flowexl (kfxmax), flowexn (kfxmax), floweyl (kfymax), floweyn (kfymax),

+ flowex (kfxmax), flowey (kflnax), enflowx (kfanax), enflowy (kfymax),

+ ennflwy (kfymax), enlflwy (kfymax), emflwx (kfxmax), enlflkx (kfxmax),

+ eneflowx (kfxmax), eneflowy (kfymax), emeflwy (kfymax),

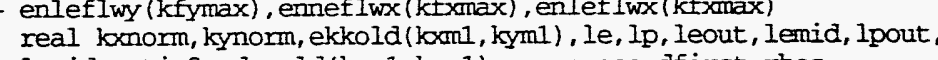

+ lpmid, ratio2, edenold (kxml, kyml), nue, emass, dfirst, rhos common /parms/ 
+ v1, gama1, gama2, epsi, le, lp, kxg, kyg, kxnorm, kynorm, kxs, kys,

+ nwrt, nwrs, $t, d t$, tol, ek, gamk, flux, ncl, ratio, psio, d, dmid, dout, loc

init. flreal, flima, ekkold, enkold, flexold, flowex, flowey, gama3,

flowexl, flowexn, flowey l, floweyn, lpmid, lpout, lemid, leout, phase,

+ flpxold, flowpx, flowpy, flowpxl, flowpxn, flowpy l, edenold, nue,

t amp, flowpyn, en, gamen, ed, et, ekold, enold, enflowx, enflowy, emass,

+ ennflwy, enlflwy, ennflwx, enlflwx, eneflowx, eneflowy, dfirst, rhos,

enneflwy, enleflwy, enneflwx, enleflwx

+ psi,den

common /arrdim/

+ nt,ntstep, pesu

real ekk (koml, kyml)

do $20 j=1, \mathrm{kyml}$

do $10 \mathrm{i}=1, \mathrm{kxm}$

$x k=\operatorname{kxmorm}^{\star}(i-\operatorname{kom} 2)$

f (ncl .le. 10) then

$\operatorname{ekk}(i, j)=\operatorname{ekkold}(i, j)$

else

ekkk $(i, j)=$ enkold $(i, j) /$ real (nwrs)

10 continue

20 continue

c write the $k^{\star \star} 2$ vs em, ek and flux in array form

do $30 \quad i=k x m 2, k x m l$
do $40 \quad j=k y m 2, k y m l$

$\mathrm{xk}=\mathrm{k} \times \mathrm{mmorm} *(i-\mathrm{kxm} 2)$

$y k=k_{y n o r m}^{*}(j-k y m 2)$

$$
a k=k^{\star \star} 2+y k^{\star \star}
$$

continue

\section{write $(14, *)$}

200 format $(50(e 12,4,1 x))$

return

end

subroutine writeer ( $h$, ier)

parameter $\left(\mathrm{kxmax}=10, \mathrm{kymax}=10, \mathrm{kom} 1=2 \star \mathrm{k} k \mathrm{mmax}+1, \mathrm{kym} \mathbf{1}=2 \mathrm{k}_{\mathrm{k} y \max }+1\right.$,

$+k \times m 2=k x m a x+1, k y m 2=k y m a x+1, n n=4 *(k x m 1 * k y m a x+k x m a x)$

$+\mathrm{kf} x \max =\mathrm{kxmax}-1, \mathrm{kfymax}=\mathrm{kymax}-1)$

complex psi (kxml, kyml), den (loxml, kymI) , flowpx (kfxmax)
+ flowpy (kfymax).

+ flowpxl (kfxmax), flowpxn (kfxmax), flowpyl (kfymax), flowpyn (kfymax),

+ flowexl (kfxmax), flowexn (kfxmax), floweyl (kfymax), floweyn (kfymax),

+ flowex (kfxmax), flowey (kfymax), enflowx (kfxmax), enflowy (kfymax),

+ ennflwy (kfymax), enlflwy (kfymax), ennflwx (kfxmax), enl

+ enleflwy (kfymax), enneflwx (kfxmax), enleflwx (kfxmax)

real kxnorm, kymorm, ekkold ( $k x m l$, kyml), le, Ip, leout, lemid, lpout,

Ipmid, ratio2, edenold ( $\mathrm{kmml}$, kyml) , nue, emass, dfirst, rhos

conmon /parns/

+ v1, gama1, gama2, epsi, le, lp, kxg, kyg, kxnorm, kynorm, kxs, kys.

+ nwIt, nwIs, $t$, dt, tol, ek, gamk, flux, ncl, ratio,psio, d, dmid, dout, loc.

+ init,flreal, flima, ekkold, emisold, flexold, flowex, flowey, gama3.

+ flowexl, flowexn, floweyl, floweyn, lpmid, lpout, lemid, leout, phase,

+ flpxold, flowpx, flowpy, flowpxl, flowpxn, flowpyl, edenold, nue,

+ amp, flowpyn, en, gamen, ed, et, ekold, enold, enflowx, enflowy, emass,

+ enneflwy, enleflwy, enneflwx, enleflwx

common /arrays/

+ psi,den

common /arrdim/

+ nt, ntstep, pesu

f (ier .eq. 33) then

'

low or equations too stiff, 'tol

write $(6, \star) \cdot ' h=\cdot, h$, ier $=\cdot$, ier, 'tol may be too

+ low or equations too stiff, $t=t+h$.

elseif (ier eq. 66 . or. ier .eq. 67) then

write $(9, *) \cdot h={ }^{\prime}, h, '$ ier $=$ ', ier, ' $h$ was decreased to

+ force convergence'

write $(6 * *)$ ' $h=$ ', $h$, ' ier=', ier, ' $h$ was decreased to

+ force convergence

else

write $(9, *)$ ' $h=', h$, 'ier=', ier, 'unknown error'

write $(6, *) \cdot h=\cdot, h$, 'ier=', ier, 'unknown error'

endif

return

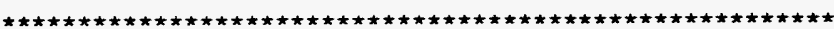

subroutine energy

parameter (kxmax $=10, \mathrm{kymax}=10, \mathrm{kxml}=2 * k \mathrm{kmax}+1, \mathrm{kyml}=2 \star \mathrm{kymax}+1$,

$+\mathrm{kxm} 2=\mathrm{kxmax}+1, \mathrm{kym} 2=\mathrm{kymax}+1, \mathrm{mn}=4$ * $\left(\mathrm{kxml}{ }^{*} \mathrm{kymax}+\mathrm{kxmax}\right)$

$+\mathrm{kfxmax}=\mathrm{k}$ (xmax $-1, \mathrm{kfymax}=\mathrm{kymax}-1)$

complex psi (kxml, kyml), den (koml, kyml), flowpx (kfxmax),

+ flowpy (kfymax), flowpxn (kfxmax), flowpyl (kfymax), flowpyn (kfymax)

+ flowexl (kfxmax), flowexn (kfxmax), floweyl (kfymax), floweyn (kfymax)

+ flowex (kfxmax), flowey (kfymax), enflowx (kfxmax), enflowy (kfymax),

+ ennflwy (kfymax), enlflwy (kfymax), ennflwx (kfxmax), enlflwx (kfxmax)

+ eneflowx (kfxmax), eneflowy (kfymax), enneflwy (kfymax)

+ enleflwy (kf, max), enneflwx (kfxmax), enleflwx (kxmax)

real kxnorm, kynorm, ekkold ( $\mathrm{kxml}, \mathrm{kyml})$, le, lp, leout, lemid,

+ lpmid, ratio2, edenold ( $\mathrm{bml}$, kyml) , nue, emass, dfirst, rhos
common / parms/ 
+ v1, gama1, gama2, epsi, le, lp, kxg, kyg, loxnorm, kynorm, kxs, kys,

+ nwrt, nwrs, t, dt, tol, ek, gamk, flux, ncl, ratio, psio, d, dmid, dout, loc

+ init, flreal, flima, ekkold, enkold, flexold, flowex, flowey, gama3,

+ flowexl, flowexn, floweyl, floweyn, lpmid, lpout, lemid, leout, phase,

+ flpxold, flowpx, flowpy, flowpxl, flowpxn, flowpyl, edenold, nue,

+ amp, flowpyn, en, gamen, ed, et, ekold, enold, enflowx, enflowy, emass,

+ ennflwy, enlflwy, ennflwx, enlflwx, eneflowx, eneflowy, dfirst, rhos,

+ enneflwy, enleflwy, enneflwx, enleflwx

common /arrays/

+ psi,den

common /arrdim

+ nt,ntstep, pesu
real emin, ekin

real enin, ekin, msfin, esin

integer pesuin

ing

c initialization

$$
\begin{aligned}
& \text { ek }=0 . \\
& \text { en }=0 . \\
& \text { ed }=0 . \\
& \text { et }=0 . \\
& \text { es }=1.0 e-25 \\
& \text { flux }=0 . \\
& \text { phase }=0 . \\
& \text { number }=0
\end{aligned}
$$

c calculate energies

$$
\begin{aligned}
& i 1=1 \\
& i 2=\text { kxmax }
\end{aligned}
$$

$j 1=\mathrm{kym} 2$

$$
j=\mathrm{kg}
$$

$$
\begin{aligned}
& x k=k x n 0 m^{*}(i-k x m 2) \\
& \text { do } 20 j=j 1, j 2 \\
& y k=k y n o n m^{\star}(j-k y m 2) \\
& a k=x k^{\star \star \star} 2+y k^{\star \star 2}
\end{aligned}
$$

ek=ek+psi $(i, j) *$ conjg $(p s i(i, j\}) * 2$

en=en+psi $(i, j) * \operatorname{conjg}(\operatorname{ps} i(i, j)) * a k^{\star}$

$\left.{ }^{\star} \operatorname{conjg}(\operatorname{psi}(i, j))\right) \star 2$

phase=phasetatan (aimag $(\operatorname{den}(i, j)) /$ real $(\operatorname{den}(i, j))$ -

+ $\quad$ phase=phasetatan (aimag (den $(i, j), j$ real

20 continue

if (xk .eq. kxmax ${ }^{\star}$ bonorm and. yk .eq. kymax*kynorm) goto 30

$i 1=\operatorname{kxm} 2$

$i 1=\mathrm{kxm} 2$
$i 2=\mathrm{kxm} 1$

$j 1=\mathrm{kym} 2+1$ et $=\mathrm{ek}+\mathrm{ed}$

apsi $i$ amax $1(($ entenold $) / 2$,es )

bpsi=amaxl ( (ek+ekold) /2,es)

gamen $=(($ en-enold $) / d t) /$ apsi

gamk $=((\mathrm{ek}-\mathrm{ekold}) / \mathrm{dt}) / \mathrm{bpsi}$

$c$ save old energies

ekold=ek

retur
end



subroutine kflowe

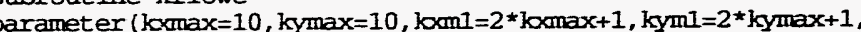

$+\mathrm{k} \times \mathrm{m} 2=\mathrm{kxmax}+1, \mathrm{kym} 2=\mathrm{kymax}+1, \mathrm{nn}=4^{*}(\mathrm{kxm} 1$ *kymax $+\mathrm{kxmax})$,

kfxmax $=$ kxmax $-1, k f y \max =k y m a x-1)$

complex psi (kxml, kyml), den (kxml, kyml) , flowpx (kfsmax) .

+ flowpy (kfymax)

flowpxl (kfxmax), flowpxn (kfxmax), flowpyl (kfymax), flowpyn (kfymax)

flowexl (kfxmax), flowexn (kfxmax), floweyl (kfymax), floweyn (kfymax)

+ flowex (kfxmax), flowey (kfymax), enflowx (kfxmax), enflowy (kfymax),

+ ennflwy (kfymax), enlflwy (kfymax), ennflwx (kfxmax), enlflwox (kfxmax)

+ eneflowx (kfxmax), eneflowy (kfymax), enneflwy (kfymax),

enleflwy (kfymax), enneflwx (kxmax),

real komorm, kynorm, ekkold ( $\mathrm{xmm}^{2}$, kyml), le, lp, leout, lemid, lpout,

+ Ipmid, ratio2, edenold ( $\mathrm{kml}$, kyml), mue, emass, dfirst, thos

common /parms/

+ vi, gamal, gama2, epsi, le, lp, loxg, kyg, knonorm, kynorm, locs, kys .

+ nwIt, nWrs, $t$, dt, tol, ek, gank, flux, ncl, ratio, psi 0 , d, dmid, dout, loc

+ init, flreal, flima, ekkold, enkold, flexold, flowex, flowey, gama3,

+ flowexl, flowexn, flcweyl, floweyn, lpmid, lpout, lemid, leout, phase.

+ flpxold, flowpx, flowpy, flowpxl, flowpxn, flowpyl, edenold, nue,

+ amp, flowpyn, en, gamen, ed, et, ekold, enold, enflowx, enflowy, emass,

enneflwy, enleflwy, emneflwx, enleflwx

cormon /arrays/

psi, den

common /arrdim/

nt, ntstep, pesu

igml) , ekoxflwe, ekyflwe, ekxloce, ekonone,

, enyeloc, enyenon, enyeflw, enxeloc, enxenon,

$1=1$

$i 2=k x m 1$

$j=120$

do $20 i=i 1, i 2$ 




do $30 \mathrm{~m}=1, \mathrm{kf}$ xmax

$k \times l=m$

call kflowe2 ( $u, v$, kxl, ekxloce, ekomone, enxeloc, enxenon)

ekxflwe=elocloce+eknone

enxefl $w=e n x e l o c+e n x e n o n$

flowexl $(m)=$ flowexl $(m)+$ ebocloce

enletlwx $(m)=e n l e f l w x(m)$ enxelo

flowexn(m) = llowexa $(m)+$ thane

enceflux (m) =enneflux (m) tenxenon

eneflowx $(m)=$ eneflowx $(m)$ +enxefl

30

continue

do $40 \mathrm{ml}=1$, kfymax

$\mathrm{ky} l=\mathrm{ml}$

call kflowel ( $u, v, k y l$, ekyloce, ekynone, enyeloc, enyenon)

ekyflwe=ekyloce+ekynone

floweyl $(\mathrm{ml})=$ floweyl $(\mathrm{mi})+$ ekyloce

enleflwy $(\mathrm{ml})=$ enleflwy $(\mathrm{ml})+$ enyeloc

floweyn $(m l)=$ floweyn $(m 1)$ +ekynone

emeflwy $(\mathrm{ml})=$ enneflwy $(\mathrm{ml})$ +enyenon

flowey $(m 1)=$ flowey $(m l)+$ ekyflwe

eneflowy (mI) =eneflowy (mI) +enyeflw

40

contimue

return

end

subroutine kflowel ( $\mathrm{u}, \mathrm{v}, \mathrm{kyl}$, ekyloce, ekynone, enyeloc, enyenon)

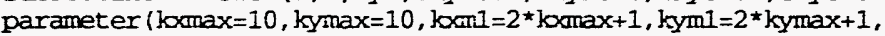

$+\mathrm{kxm} 2=\mathrm{kxmax}+1, \mathrm{kym} 2=\mathrm{kymax}+1, \mathrm{mn}=4$ * (kxm1 *kymax $+\mathrm{kxmax})$,

$+\mathrm{kfxmax}=\mathrm{k}$ comax $-1, \mathrm{kfymax}=\mathrm{kgmax}-1)$

complex psi (kxml, kyml), den (koml, kyml), flowpx (kfxmax)

flowpy (kfymax)

+ flowpxl (kfxmax) , flowpxn (kfxmax), flowpyl (kfymax), flowpyn (kfymax)
+ flowexl (kfxmax), flowexn (kfxmax), floweyl (kfymax), floweyn (kfymax), + II wex (kfxmax), flowey (kfymax), enflowx (kfxmax), enflowy (kfymax),

( eneflux (kfyax), ent

real kononm, kynorm, ekkold (koml, kymi), le, lp, leout, lemid, lpout,

+ lpmid, ratio2, edenold (kxml, kyml), mue, emass, dfirst, thos

common /parms/

+ v1, gamal, gama2, epsi, le, lp, kxg, kyg, komorm, kynorm, kxs, kys,

+ mwrt, nwrs, $t$, dt, tol, ek, gamk, flux, ncl, ratio,psio, d, dmid, dout, loc,

+ init, flreal, flima, ekkold, enkold, flexold, flowex, flowey, gama3.

+ flowexl, flowexn, fl oweyl, floweyn, Ipmid, I pout, Iemid, leout, phase,

+ flpxold, flowpx, flowpy, flowpxl, flowpon, flowpyl, edenold, me,

+ amp, flowpyn, en, gamen, ed, et, ekold, enold, enflowx, enflowy, emass,

+ ennflwy, enlflwy, emfliwx, enlflwx, eneflowx, eneflowy, dfirst, chos,

+ emeflwy, enleflwy, enneflwx, enleflwx

cormon /arrays/

+ psi,den

cormon /arrdim/



+ enyeloc, enyenon, $v$ (koml, $k y m$ )

integer $\mathrm{kyl}$

ekyloce $=(0,0$.

ekynone $=(0 ., 0$.

enyeloc $=(0 ., 0$.

enyenon $=(0 ., 0$

$i c=(0 ., 1$

$11=1$

i2 $=1$

$j 1=1$

do $20 i=i 1, i 2$

do $20 \quad j=j 1, j 2$

kyY $=j-\mathrm{kym} 2$

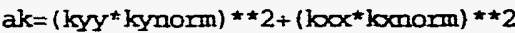

if (iabs (kyy) .le. kyl) then

call yflowe (u, v, kyl, kox, kyy, dny loce, dnynone)

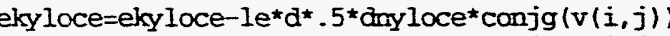

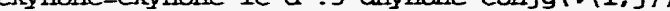
enyenon=enyenon-le* $\mathrm{d}^{\star}, 5^{\star} a k^{\star}$ dnymone $e^{\star} \operatorname{conjg}(v(i, j))$

endif continue

if (krox .eq. kxmax and. kyy .eq. kymax) goto 30 i1 $=\mathrm{kom} 2$

i2 $=$ kxmI

$j 1=2$ 


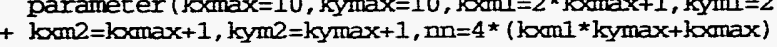

$+\mathrm{kfxmax}=\mathrm{k} x \max -1, \mathrm{kfy}$ max $=\mathrm{kymax}-1)$

complex psi (kxml, kyml), den (kxml, kymI) , flowpx (kfxmax),

+ flowpy (kfymax).

+ flowpxl (kfxmax), flowpxn (kfxmax), flowpyl (kfymax), flowpyn (kfymax)

+ flowexl (kfxmax), flowexn (kfxmax), floweyl (kfymax), floweyn (kfymax)

+ flowex (kfxmax), fiowey (kfymax), enflowx (kfxmax), enflowy (kfymax),

+ ennflwy (kfymax), enlflwy (kfymax), ennflwx (kfxmax), enl
+ eneflowx (kfxmax), eneflowy (kfymax), enneflwy (kfymax).

+ enleflwy (kfymax), enneflwx (kfmax), enleflwx (keout, lemid, lpout.

+ lpmid, ratio2, edenold ( $\mathrm{kml}$, kyml), nue, emass, dfirst, rhos

common /parms/

+ v1, gamal, gama2, epsi, le, lp, kxg, kyg, bx,orm, kynorm, kxs, kys,

+ nwTt, nwrs, t, at, tol, ek, gamk, flux, ncl, ratio, psio, d, dmid, dout, loc.

+ init,flreal, flima, ekkold, enkold, flexold, flowex, flowey, gama3,

+ fipxold, flowpx, flowpy, flowpxl, flowpxn, flowpyl, edenold, nue,

+ amp, flowpyn, en, gamen, ed, et, ekold, enold, enflowx, enflowy, emass

+ ennflwy, enlflwy, ennflwx, enlflwx, eneflowx, eneflowy, dfirst, rhos

+ enneflwy, enleflwy, enneflwx, enleflwx

common /arrays/

+ psi, den

common /arrdim/

+ nt, ntstep, pesu

complex u (kxml, kyml), dnxloce, dnxnone, ekxloce, ekxnone, ic,

+ enxeloc, enxenon, $v$ ( $\mathrm{koml}, \mathrm{kyml})$

integer kol

ekxloce $=(0 ., 0$.

elonone $=\left(0,0^{-},{ }^{2}\right.$

enxeloc $=(0 ., 0$.

enxenon $=(0 ., 0$.

ic $=(0 ., 1$.)

$i 1=1$

i2 $=$ kxmax

$j 1=1$

do $70 i=i 1, i 2$

$\mathrm{kxx}=i-\mathrm{kxm} 2$

do $70 j=j 1$, $j 2$

kyy $=j-1$

$a k=\left(k y y^{\star} k y n o r m\right) * \star 2+\left(k x x^{\star} k \times n o r m\right) \star \star 2$

if (iabs $(k \infty x)$. le. $k x I)$ then call xflowe $(u, v, k x l$, lox, kyy, dinxloce, dnomone)

ekxloce =elocioce-le $e^{\star} d^{\star} \cdot 5^{\star}$ dnxloce $\operatorname{conjg}(v(i, j+k y$ max $j)$ )

elomone $=e k x n o n e-1 e^{\star} d^{\star} \cdot 5^{\star}$ droxnone* $\operatorname{conjg}(v(i, j+k y$ max $))$

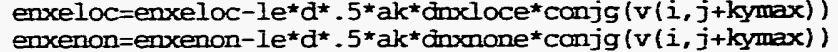

endif

70

if (kxx .eq. bomax .and. kyy .eq. kymax) goto 80

$i 1=\mathrm{k} \times \mathrm{m} 2$

i2 $=$ kom

$j 1=2$

goto 60

80

return

end

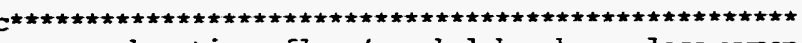

subrout ine yflowe ( $u, v, k y l, k x x, k y y$, wyloce, wynone)

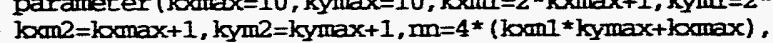

$+\mathrm{kfxmax}=\mathrm{k}$ mmax $-1, \mathrm{kfymax}=\mathrm{kymax}-1)$

camplex psi (koml, kyml), den (koml, kymil, flowpx (kfxmax),

flowpy (kfymax).

+ flowpxl (kfxmax), flowpon (kfxmax), flowpyl (kfymax) , flowpyn (kfymax).

+ flowexl (kfomax), flo exon (kffmax), floweyl (kfymax), floweyn (kfymax).

+ flowex (kfxmax), flot, (kfymax), enflowx (kfxmax), enflowy (kfymax).

+ emflwy (kfymax), enlflwy (kfymax), ennflwax (kfxmax), enlflwx (kfomax),

+ eneflowx (kfxmax), eneflowy (kfymax), enneflwy (kfymax),

+ enleflwy (kfymax), enneflwx (kfmax), enleflwx (kfumax)

real $\mathrm{kxnorm}, \mathrm{kynorm}$, ekkold (kxml, kymI), le, lp, leout, lemid, lpcut,

Ipmid, ratio2, edenold (koml, kyml) , mue, emass, dfirst, rhos

common /parms/

+ v1, gamal, gama2, epsi, le, lp, koxg, kyg, komorm, kynorm, loxs, kys.

+ mwrt, nwrs, $t$, dt, tol, ek, gamk, flux, ncl, ratio, psi 0, d, dmid, dout, loc

+ init,flreal, flima, ekkold, enkold, flexold, flowex, flowey, gama3.

+ flowexl, flowexn, floweyl, floweyn, lpmid, lpout, lemid, leout, phase,

+ flpxold, flowpx, flowpy, flowpxl, flowpxo, flowpyl, edenold, me,

+ amp, flowpyn, en, gamen, ed, et, ekold, enold, enflow, enflowy, enass,

+ ennflwy, enlflwy, ennflwx, enlflwx, eneflowx, eneflowy, dfirst, thos,

+ emeflwy, enleflwy, emeflwx, enleflwx

cormon /arrays/

+ psi,den

common /arrdim/

complex u (koml, kymI) , v (kxml, kymi) , wyloce, wy wone,

+ wylloce, wylnone

integer kyl

$c$ intialization

c process 
wyloce $=(0.0,0.0)$

wys

$$
\begin{aligned}
& i 1=1
\end{aligned}
$$

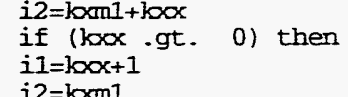

$i 1=100 x+1$
$i 2=k \times m I$

end if

\section{$j 1=1$}

$j 2=k y m I+k y y$

if (kyy .gt. 0) then

$j 1=k y y+i$

$j \hat{2}=\mathrm{kyml}$
endif

do $20 i=i 1, i 2$

$20 j=j 1, j 2$

$\mathrm{kx} 1=i-\mathrm{kxm} 2$

$\mathrm{ky} 1=\mathrm{j}-\mathrm{kym} 2$

kymod=koor-koci

$\mathrm{kom}=\mathrm{k} \times \mathrm{m} 2+\mathrm{kxmod}$

kym=kym2 +kymod

if ( ( (iabs (ky1) - gt. kyl) .or. (iabs (kyy-ky1) .gt. kyl)) . and.

+ ((iabs (kyl-kyl). le.loc).or. (iabs ( (kyy-ky1)-kyl). le.loc)) then

c convolute

wy1loce $=(u(i, j) * v(k x m, k y m)-u(k x m, k y m) * v(i, j))$

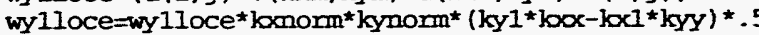

wyloce $=$ wyloce + wylloce

elseif(((iabs (kyl) .gt. kyl) .or. (iabs (kyy-kyl) .gt. kyl)) .and.

$+((i a b s(k y 1-k y 1) \cdot g t .10 c)$.or. (iabs $((k y y-k y 1)-k y l)$.gt. loc $)))$ then

wylnone $=\left(u(i, j){ }^{*} v(\mathrm{kom}, \mathrm{kym})-u(\mathrm{kom}, \mathrm{kym}){ }^{*} v(i, j)\right)$

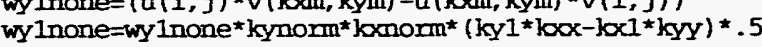
endif

20

\begin{abstract}
continue
\end{abstract}
return

end

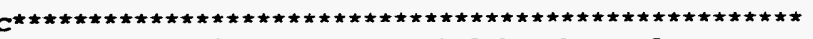



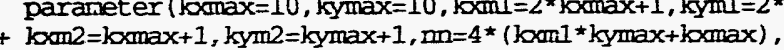

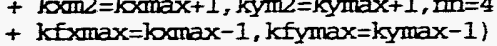

complex psi (koml, kyml), den (kxm1, kyml), flowpx (kf fmax)

+ flowpy (kfymax).

+ flowpxl (kfumax) , flowpxn (kfxmax), flowpyl (kfymax) , flowpyn (kfymax),
+ flowexl (kfumax), flowexn (kfxmax), floweyl (kfymax), floweyn (kfymax),

+ flowex (kfxmax), flowey (kfymax), enflowx (kfxmax), enflowy (kfymax),

+ ennflwy (kfymax), enlflwy (kfymax), ennflwx (kfmax), enlflwo (kfomax).

+ eneflowx (kfxmax), eneflowy (kfymax), emeflwy (kfymax).

real kxnorm, kynorm, ekkold (komi, kyml), le, lp, leout, lenid, lpout.

+ Ipmid, ratio2, edenold ( $k 0 m 1, k y m 1$ ) mue, emass, dfirst, thos

carmon /parms/

+ v1, gamal, gama2, epsi, le, lp, kxg, kyg, bomorm, kymorm, locs, kys,

+ mwrt, nwrs, t, dt, tol, ek, gamk, flux, ncl, ratio, psio, d, dmid, dout, Ioc

+ init, flreal, flima, ekkold, enkold, flexold, flowex, flowey, gama3.

+ flowexl, flowexn, floweyl, floweyn, Ipmid, Ipout, lemid, leout, phase,

+ flpxold, flowpx, flowpy, flowpxl, flowpso, flowpyl, edenold, nue,

+ amp, flowpyn, en, gamen, ed, et, ekold, enold, enflowx, enflowy, enass,

+ emeflwy, enleflwy, enneflwx, enleflwx

common /arrays/

+ psi,den

calmon /arrdim

+ nt, ntstep, pesu

camplex $u(k x m 1, k y m l), v(k x m l, k y m l)$, wxloce, wxmone,

wxiloce, wxinon

$c$ intialization

$c$ process

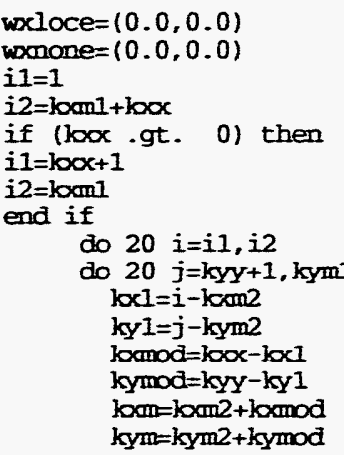

wxloce $=(0.0,0.0)$

$=(0.0,0.0)$

il $=1$

$\mathrm{i} 2=\mathrm{k} \times \mathrm{m} n+\mathrm{k} \times \mathrm{x}$

if $($ loox .gt. 0$)$ then

$\mathrm{i} 1=\mathrm{k} \times \mathrm{x}+1$

i2 $=\mathrm{k} \times \mathrm{mm}$

do

$20 i=i 1, i 2$

do $20 j=k g y+1$,

$\mathrm{kxl}=i-\mathrm{kom} 2$

kcmod=k00x-koct

kymod=kyy-ky1

$\mathrm{kmm}=\mathrm{k} \times \mathrm{xm} 2+\mathrm{kxmod}$

kym=kym $2+$ kymod

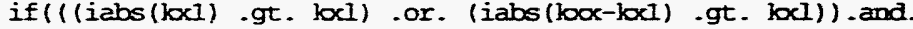

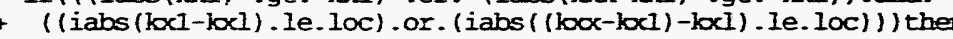

c convolute

$w \times 1 l o c e=(u(i, j) \star v(k \times m, k y m)-u(k x m, k y m) * v(i, j)$

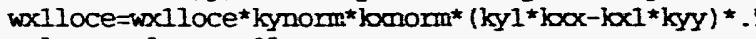
wxloce=wxioce+wxiloce

elseif(((iabs (kocl) .gt. $k x d)$.or. (iabs (kox-kocl) -gt. boll)) and $+(($ iabs $(k \times 1-k \times 1)$.gt. Ioc) . or. (iabs ( (koox-kocl)-kocl) . gt. loc) i) then 
$w x I n o n e=(u(i, j) * v(b x m, k y m)-u(k x m, k y m) * v(i, j))$

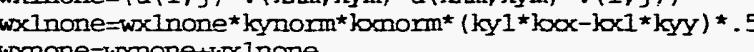

return

end subroutine writflwe

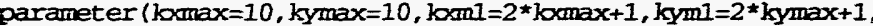

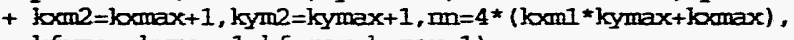

+ kfxmax $=k x m a x-1$, kfymax $=k y$ max -1

camplex psi (koml, kyml), den (kxml, kyml) , flowpx (kñmax),

+ flowpy (kfymax)

+ flowpxl (kfxemax), flowpxn (kfxmax), flowpyl (kfymax), flowpyn (kfymax)

+ flowexl (kfxmax), flowexn (kfumax), flowey l (kfymax), floweyn (kfymax)

+ flowex (kfxmax), flowey (kfymax), enflowx (kfxmax), enflowy (kfymax),

+ ennflwy (kfymax), enlflwy (kfymax), ennflwx (kfmax), enlflwo (kfxmax)

+ eneflowx (kffmax), enefl cwy (kfymax), enneflwy (kfymax)

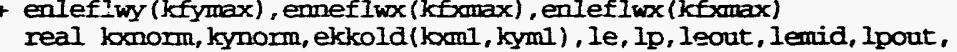

+ Ipmid, ratio2, edenold (koml, kyml), mue, emass, dfirst, thos

+ camid, ratio2, eden

+ v1, gamal, gama2, epsi, le, lp, kxg, kyg, locmorm, kynorm, loxs, kys.

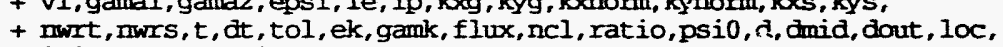

+ mint, Ifrs, t, dt, tol, ek, gank, flux, ncl, ratio, psio, , dmid, dout,

+ flowexl, flowexn, floweyl, floweyn, lpmid, l pout, lemid, leout, phase

+ flpxold, flowpx, fl lowpy, flowpxl, fl owpxn, flowpyl, edenold, me.

+ amp, flowpyn, en, gamen, ed, et, ekold, enold, enflowx, enflowy, emass,

+ emflwy, enlflwy, ennflwx, enlflwx, eneflowx, eneflowy, dfirst, thos.

enneflwy, enleflwy, enneflwx, enleflwx

+ psi,den

carmon /arrdim/

+ nt,ntstep, pesu

real flowerx (kfxmax), floweix (kfumax), flowery (kfymax)

+ floweiy (kfymax), flwerxl (kfxmax), flweron (kfxmax), flweixl (kfxmax)

+ flweixn (kfomax), flweryl (kfymax), flweryn (kfymax),

+ flweiyl (kfynax), flweiyn (kfymax)

do $30 \mathrm{~m}=1$, kf $x$ max

flowerx $(\mathrm{m})=$ real (flowex (m) ) / real (nwIs)

floweix $(m)=$ real (eneflowx (m)) /real (Iwrs)

flwerxl (m) =real (flowexl (m)) / real (nwrs)

flwerxn(m) =real (Ilowexn(m)/real (nwrs)

flweixl (m) =real (enleflwx(m)/real (IwIS) do $50 \mathrm{mI}=1$, kfymax

flowery $(\mathrm{ml})=$ real (flowey (mi)) $/$ real (nwrs)

floweiy (ml) =real (eneflowy (mI) ) / real (mwrs)

flweryl (ml) =real (floweyl (ml)/real (nwrs)

flweryn (mil) =real (floweyn (ml) / / real (nwrs)

flweiyl (ml) =real (ennefiwy (ml))/real (mwrs)

flweiyn (ml) =real (enneflwy (ml)) / real (nwrs) contimue

write $(12, *)$ (flowerx (m), $m=1, k f$ max $),($ flowery $(m), m=1, k f y m a x)$,

+ (flwerxl (m), $\mathrm{m}=1, \mathrm{kfxmax}$ ), (flweryl (m), $\mathrm{m}=1, \mathrm{kfymax}$ )

write $(i 3, *)$ ( (floweix $(m), m=1, k f y m a x),($ (floweiy $(m), m=1$, kfymax).

( $f$ lweixl (m), $m=1, k f(m a x)$ ( $f$ Iweiy $(m), m=1, k f y$ max)

+ (flueion (m),

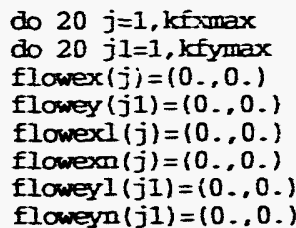

eneflowx $(j)=(0 ., 0.4)$

enleflwx $(j)=(0 . ., 0$.

enneflwx $(j)=(0 \ldots$.

eneflowy $(j I)=10 . .0$.

enleflwy $(j 1)=10 ., 0$.

enneflwy $(j 1)=10 ., 0$.

continue

format $(50(e 12.4,1 x))$

end

subroutine kflowp

parameter (bomax $=10$, kymax $=10, \mathrm{kxml}=2 *$ komax $+1, \mathrm{kyml}=2 *$ kgmax +1

$+\mathrm{k} 0 \mathrm{~m} 2=\mathrm{k} x \mathrm{max}+1, \mathrm{kym} 2=\mathrm{kymax}+1, \operatorname{mm}=4 *(\mathrm{koml} * \mathrm{ky} \max +\mathrm{komax})$,

$+\mathrm{kfxmax}=\mathrm{kogmax}-1, \mathrm{kfymax}=\mathrm{kymax}-1$ )

complex psi (koml, kyml), den (kxml, kyms), flowpx (kfxmax).

+ flowpy (kfymax)

+ flowpol (kfxmax), flowpon (kfxmax), flowpyl (kfymax), flowpyn (kfymax)

+ flowexl (kfomax), flowexn (kfxmax), flcweyl (kfymax), floweyn (kfymax),

+ flowex (kxmax), flowey (kfymax), enflowx (kfomax), enflowy (kfywax),

+ emflwy (kfymax), enlflwy (kfymax), emflwxx (kfxmax), enlflwx (kfxmax)

+ eneflowx (kmax), eneflowy (krymax), emeflwy (kfymax).

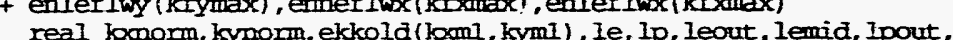

+ Ipnid, ratio2, edenold (koml, kyml), nue, emass, dfirst, rhos 
cormon /parms/

+ vl, gamal, gama2, epsi, le, lp, kxg, kyg, konorm, kynorm, koss, kys.

+ mwrt, nwrs, $t$, dt, tol, ek, gamk, flux, ncl, ratio, psio, d, dmid, dout, loc.

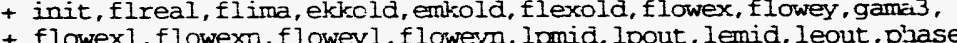

+ flpxold, flowpx, flowpy, flowpxl, flowpxn, flowpyl, edenold, nue,

+ amp, flo, en, gamen, ed, et, ekold, enold, enflowx, enflowy, emass,

+ ennflwy, enlflwy, ennflwx, enl flwx, eneflowx, eneflowy, dfirst, rhos.

+ enneflwy, enleflwy, enneflwx, enleflwx

+ psi,den

comon /arrain/

+ nt,ntstep, pesu

complex u (koml, kyml) , eloxflwp, ekyflwp, ekxlocp, ekxnonp,

+ ekylocp, ekynomp, enyloc, enynon, enyflw, enxloc, enxmon, enxflw

il $=1$

$j 1=1$

$\mathrm{j} 2=\mathrm{kym}$

do $20 i=i 1, i 2$

$$
\text { do } 20 j=j i, j 2
$$$$
\text { continue }
$$

do $30 \mathrm{ml}=1$, kfymax

$\mathrm{kyl} l=\mathrm{ml}$
$\mathrm{call} \mathrm{k} f \mathrm{lowpl}$ ( $\mathrm{u}, \mathrm{ky} \mathrm{l}$, ekylocp, ekynomp, enyloc, enynon)

ekyflwp=ekylocptekynon

enyflw-enylocterynon

flowpyl $(\mathrm{ml})=$ flowpyl $(\mathrm{ml})+$ ekylocp

flowayn (ml) =flowprn (m)

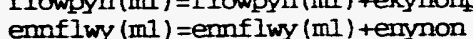

flowpy (mI) =flowpy (mI) +ekflup

enflowy (ml) =enflowy (mI) enyflw

continue

do $40 m=1$, kfxmax

\section{$\mathrm{bocl}=\mathrm{m}$}

call kflowp2 ( $u, k x l$, ekxlocp, ekonorm, enxioc, enomon)

elox I $w p=e$ bxlorptekononp

enxflw=enxiloctenxnon

flowpxl $(m)=$ flowpxl $(m)$ +eloxlocp

enlflwx $(m)=e n l f l w x(m)$ enxloc

flowpxn $(m)=f$ lowpxn $(m)$ +elomong

flowpx $(m)=$ flowpox $(m ;+$ eloxflwp

$\operatorname{enflowx}(m)=\operatorname{enf} \operatorname{Iow}(m)+\operatorname{enxfl} w$

$$
\text { continue }
$$

return

end

subrout ine kflowpl ( $\mathrm{u}, \mathrm{kyl}$ l, eky locp, ekymomp, eny loc, entmon)

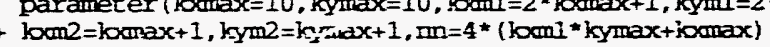

$+\mathrm{kfxmax}=\mathrm{kmmax}-1, \mathrm{kfymax}=\mathrm{kymax}-1)$

complex psi (kocol, kyml), den (koml, kyml), flowpox (kfowax)

+ flowpy (kfymax).

+ flowpxl (kfxamax), flowpon (kfomax), flowpyl (kfymax) , flowpyn (kfymax),

+ flowexi (kfxmax), flowexn (kfxmax), floweyl (kfymax), floweyn (kfymax),

+ flowex (kfxmax), flowey (kfymax), entlowx (kfxmax), enflowy (krymax).

+ eneflowx (kfsmax), eneflowy (kfymax), emeflwy (kfymax).

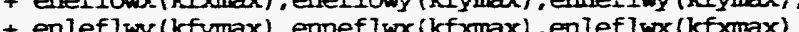

real lomorm, kynorm, ekkold (boml, kymi). Ie, ip. Ieout. Ienid, lpout.

+ 1pmid, ratio2, edenold (koml, kyml), mue, emass, dfirst, thos

common /parms/

+ v1, gamal, gama2, epsi, le, lp, kxg, kyg. bomorm, kynorm, kxs, kys,

+ nwrt, nwrs, $t$, dt, tol, ek, gamk, flux, ncl, ratio, psio, d, dmid, dout, Ioc

+ init, flreal, flima, ekkold, enkold, flexold, flowex, flowey, gama3,

+ flawexl flowern flowerl flowegn, l mid lpout lemid leout phase.

+ flpxold, flowpx, Elowpy, flowpxl, flowpon, flowpyl, edenold, nue,

+ amo, flowpym, en, gamen, ed, et, ekold, enold enflowx, enflowy, emass,

+ emflwy, enlflwy enflwx, eniflwx, enef lawx, eneflowy, juirst thos.

+ enneflwy, enlefIwy, enneflwx, enlefIwx

common /arrays/

+ psi,den

cormon /arrdim

+ nt.ntstep.pesu

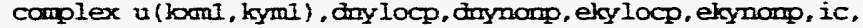

+ enyloc, enynon

integer kyl

ekylocp $=(0 . .0$.

ekynomp $=(0 . .0$.

enyloc $=(0 ., 0$.

enynon $=10 ., 0.1$

ic $=10 ., 1$

$i 1=1$

$i 2=100$

$j 1=1$

$j 2=k y m l$

10

do $20 i=i 1$, i

jo $20 j=j i$

do $20 j=j 1$ 
$a k=\left(k y y y^{\star}\right.$ hynorm $) \star \star 2+\left(k x x^{\star}\right.$ jonorm $) \star \star 2$

if (iabs (kyy) .le. kyl) then

call yflowp (u, u, kyl, box, kyy, dny locp, daynomp)

ekylocp $=$ eky locp $-1 p^{*} \mathrm{~d}^{*} .5^{*}$ dnylocp* conjg $(u(i, j))$

$+/\left(1 .+a k^{\star}\right.$ rhos $\left.{ }^{\star} \star 2\right)$

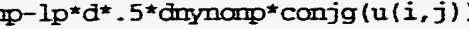

$+1\left(1 .+\right.$ ak $^{\star}$ thos $\left.\star * 2\right)$

enyloc=enyloc-lp*d* $.5^{\star} a k^{\star}$ caylocp*conjg $(u(i, j))$

$+/\left(1 .+a k^{\star}\right.$ thos $\left.\star 2\right)$

enymon=enymon- $1 p^{\star} d^{\star} \cdot 5^{\star} a^{*}$ dinynomp $p^{*} \operatorname{conjg}(u(i, j))$

$+/\left(1 .+a^{\star}\right.$ rhos $\left.^{\star \star} \approx 2\right)$

endif

20 contimue

c if (kxx .eq. kxmax and. kyy .eq. kymax) goto 30

$i 1=\operatorname{lom} 2$

goto 10

30 return

end

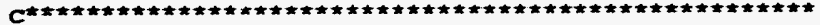

subrout ine kflowp2 ( $\mathrm{u}$, loxi, eloclocp, elomom, enoloc, enomon)

parameter (

+ bom2=komax $+1, k y m 2=k y m a x+1, m=4$ * $($ bom 1 *kymax + bomax $)$,

$+\mathrm{kfx}$ max $=\mathrm{lomax}-1, \mathrm{kfy}$ max $=\mathrm{kymax}-1)$

complex psi (boml, kyml) , den (koml, kyml) , flowpox (kfxmax),

+ flowpy (kfymax)

+ flowpxl (kfxmax), flowpon (kfxmax) , flowpyl (kfymax), flowpyn (kfymax)

flowexl (kfxmax), flowexn (kfamax), floweyl (kfymax), floweyn (kfymax)

flowex (kfxmax), filowey (kfymax), enfl cwax (kfmax), enflowy (kfymax),

+ ennflwy (kfyxax), enlflwy (kfymax), ennflwx (kfxmax), enl flwox (kfomax)

+ eneflowx (kfxmax), eneflowy (kfymax), ermeflwy (kfymax)

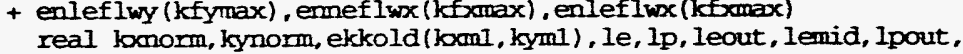

real bonorm, kynorm, ekkold (koml, kyml), le, lp, leout, lemid,
lpmid, ratio2, edenold (kxml, kyml), mue, emass, dfirst, nhos

caumon /parms'
+ v1, gamal, gama2, epsi, le, Ip, kxg, kyg, lomorn, kynorm, loxs, kys,

+ mwrt, mwrs, $t$, dt, tol, ek, gamk, flux, ncl, ratio, psio, d, dmid, dout, loc,

+ init, flreel, fl ima, ekkold, enkold, flexold, flowex, flowey, gama3.

+ flowexl, fl uwexn, floweyl, flowey, lowid, lpout, lemid, leout, phase,

+ amp, flowpyn, en, gamen, ed, et, ekold, enold, enflowx, enflowy, emass.

+ enflwy, enlelwy, ennflwx, enifliw, eneflow

+ encelwy, enleflwy, enneflwx, enleflwx

+ psi,den common /arrdim/



+ enxloc, envonon:

integer bol

eloclocp $=(0 ., 0$.

elomomp $=(0 ., 0$.

encloc $=(0 ., 0$.

emonon $=(0 ., 0$.

ic $=(0,1$.

i $1=1$

i2 $=$ lomax

$j 1=1$

60

$2=k y m 2$

do $70 i=i 1$, i2

box $=i-\operatorname{locm} 2$

do $70 j=j 1, j 2$

$\mathbf{k y y}=j-1$

$a k=\left(k y y^{*} k y n o r m\right) * * 2+(k 0 x * l o m o r m) * * 2$

if (iabs (loox). .le. boI) then

call xflowp (u, u, kocl, kox, kyy, dexiocp, dnomomp)

eloclocp=eloxlocp-Ip*d* $5^{*}$ danxlocp*conjg (u (i,j+kymax) )

$+/\left(1 .+a k^{*}\right.$ thos**2)

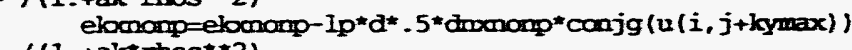



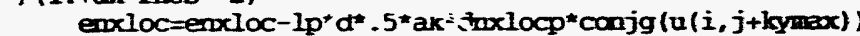

enxloc=enxil

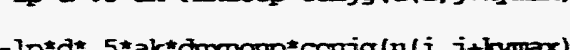

erromon=entonas

endif

70 continue

if (box .eq. lomax .and. kyy .eq- kymax) goto 80

$i 1=\operatorname{locon} 2$

$j 1=2$

goto 60

30

carn

end

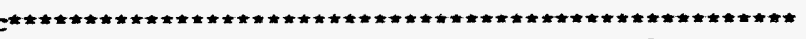

subrout ine yflowp (u, v, kyl, kox, kyy, wylocp, wymoop)

parameter $(\mathrm{kcmax}=10, \mathrm{kymax}=10, \operatorname{logal}=2 *$ bomax $+1, \mathrm{kyml}=2 * \mathrm{kymax}+1$,

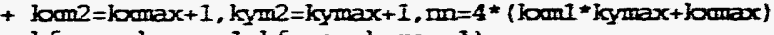

camplex psi (kaml, kyml), den (koml, kyml), flowpx (kfomax).

flowpxl (kfxmax) , flowpon (kfluax) , flowpyl (kfymax) , flowpyn (kfymax). 


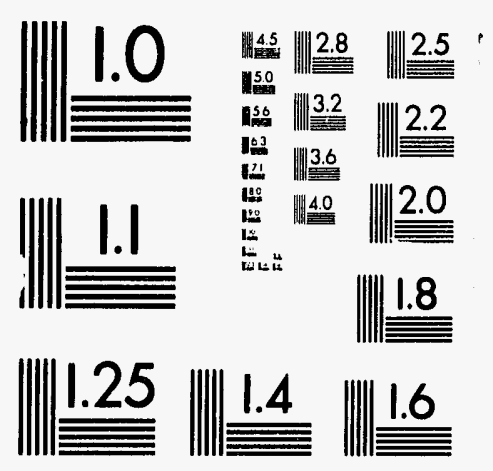



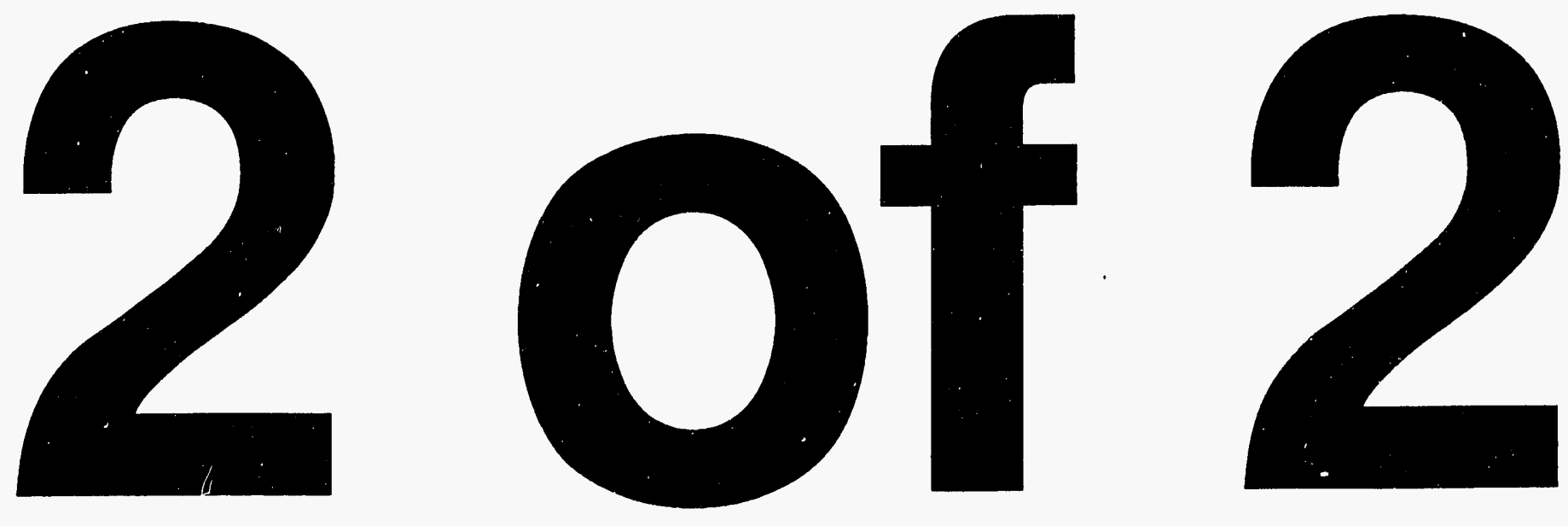
c intialization

c process

$$
\begin{aligned}
& \text { wxlocp }=(0.0,0.0) \\
& \text { wxononp }=(0.0,0.0) \\
& i 1=1 \\
& i 2=\mathrm{k} x \mathrm{~m} I+\mathrm{k} \times \mathrm{x} x \\
& \text { if (kxx gt. 0) then } \\
& \text { i } 1=\mathrm{k} 0 \mathrm{x} x+1 \\
& \text { i2 }=\mathrm{kxml} \\
& \text { if } \\
& \begin{array}{ll}
\text { do } 20 & i=i 1, i 2 \\
\text { do } 20 & j=k y y+1, k y m l
\end{array}
\end{aligned}
$$

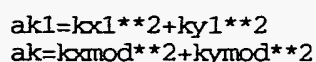

if $(((\mathrm{iabs}(k \times 1)$.gt. $k \times 1)$.or. (iabs $(k \times x-k \times 1)$. gt. $k \times 1))$, and

$+(($ iabs $(k \times 1-k \times 1)$. Ie. Ioc $)$.or. (iabs $((k \times x-k \times 1)-k \times 1)$. le. loc $)))$ then

c convolute

wxllocp $=(u(i, j) * v(k x m, k y m) * a k l-u(k x m, k y m) * v(i, j) *$

$+\quad a k) \star k \times n o r m * k y n o r m$

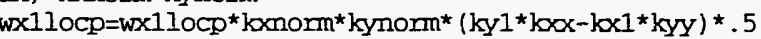

wxlocp $=w \times 10 c p+w \times 110 c p$

elseif(((iabs (kxl) .gt. kxl) .or. (iabs (kxx-kxl) .gt. kxl)) and.

$+\quad(($ iabs $(k \times 1-k \times 1) \cdot g t$. Ioc) or. (iabs $((k \times x-k \times 1)-k \times 1) \cdot g t .10 c)))$ then

wxlnonp $=(u(i, j) * v(k x m, k y m) * a k l-u(k x m, k y m) * v(i, j)$ *

$\mathrm{ak}) \star \mathrm{kxnorm} \star \mathrm{kynorm}$



20

continue

return

end

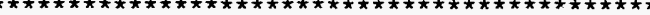

subroutine writflwp

parameter (kxmax $=10$, kymax $=10, \mathrm{kxm1}=2$ *komax $+1, \mathrm{kyml}=2 \star \mathrm{kymax}+1$

$+\mathrm{kxm2}=\mathrm{kxmax}+1, \mathrm{kym} 2=\mathrm{kymax}+1, \mathrm{nn}=4$ * $(\mathrm{kxm} 1 * k y \max +\mathrm{kxmax})$,

complex psi (kxml, kyml), den (kxml, kyml) , flowpx (kfxmax).
+ flowpy (kfymax)

flowpxl (kfxmax), flowpxn (kfxmax) , flowpyl (kfymax) , flowpyn (kfymax)

flowexl (kfxmax), flowexn (kfxmax), floweyl (kfymax), floweyn (kfymax)

flowex (kfxmax), flowey (kfymax), enflowx (kfxmax), enflowy (kfymax).

+ ennflwy (kfymax), enlflwy (kfymax), ennflwx (kfxmax), enlflwx (kfxmax)

+ eneflowx (kfxmax), eneflowy (kfymax), enneflwy (kfymax)

+ enleflwy (kfymax), enneflwx (kfxmax), enleflwx (kfxmax)

+ lpmid, raitio2, edenold (koml, kyml), nue, emass, dfirst, rhos

conmon /parms/

+ v1, gama1, gama2, epsi, le, lp, kxg, kyg , kxnorm, kynorm, kxs, kys,

+ nwrt, nwrs, $t, d t$, tol, ek, gamk, flux, ncl, ratio, psi0, d, dnid, dout, loc,

+ init, flreal, flima, ekkold, emkold, flexold, flowex, flowey, gama3,

+ flpxold, flowpx, flowpy, flowpxl, flowpxn, flowpyl, edenold, nue,

+ Ilpxold, flowpx, flowpy, flowpxl, flowpxn, flowpy, edenold, nue,

+ amp, flowpyn, en, gamen, ed, et, ekold, enold, enf enef, ewy, dfirst, rhos

+ enneflwy, enleflwy, enneflwx, enleflwx

comon /arrays/

+ psi,den

common /arrdim/

+ nt, ntstep, pesu

real flowprx (kfxmax), flowpix (kfxmax), flowpry (kfymax)

+ flompiy (kfymax) , flwprxl (kfxmax) , flwpron (kfxmax), flwpixl (kfxmax)

flwpixn (kfxmax), flwpryl (kfymax), flwpryn (kfymax)

flwpiyl (kfymax), flwpiyn (kfymax)

do $30 \mathrm{~m}=1$, kf $x$ max

flowprx (m) =real (flowpx(m)) /real (nwrs)

flowpix $(m)=$ real (enflowx $(m)$ ) $/$ real (nwrs)

fluprxl $(m)=$ real (flowpxl (m))/real (nwrs)

flwprxn $(m)=$ real (flowpxn(m))/real (nwrs)

flwpixl $(m)=$ real (enlflwx(m))/real (nwrs)

flwpixn $(m)=r e a l(e n n f l w x(m)) / r e a l$ (nwrs)

continue

do $50 \mathrm{ml}=1, \mathrm{kfymax}$

flowpry (ml) =real (flowpy (ml)) / real (nwrs)

flowpiy (ml) =real (enflowy (ml)) / real (nwrs)

flwpry $1(\mathrm{ml})=$ real (flowpyl (mI) /real (nwrs)

flwpryn (m1) =real (flowpyn (m1) /real (nwrs)

flwpiyl (ml) =real (enlflwy (m1))/real (nwss)

50 continue

write $(15, *)$ (flowprx $(m), m=1, k f x m a x)$ ( flowpry (m), $m=1, k f y m a x)$

(flwprxl (m), $m=1, k f x m a x)$, (flwpryl (m), $m=1$, kfymax),

(flwprxn(m), $m=1, k f x m a x),(f l w p r y n(m), m=1, k f y m a x)$.

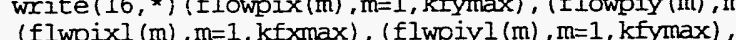

do $20 j=1, k f x m a x$

do $20 j 1=1, k f y$ max 
flowpx $(j)=(0, ., 0$.

flowpy $(j I)=(0,0$.

flowpxi $(j)=(0 ., 0$.

flowpxen $(j)=(0 ., 0$.

flowpy $1(j 1)=10$

flowpyn $(j 1)=(0 ., 0$.

enflowx $(j)=(0 ., 0$.

enlflwx $(j)=(0 ., 0$.

ennflwx $(j)=(0 ., 0$.

enlflwy $(j 1)=(0.0$.

ennflwy $(j 1)=(0 . .0$.

20

continue

200

format $(50(e 12.4,1 x))$

return

end

subroutine pert

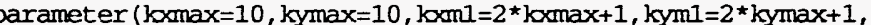

$+\mathrm{kxm} 2=\mathrm{kx}$ max $+1, \mathrm{kym} 2=\mathrm{kymax}+1, \mathrm{nn}=4$ * $(\mathrm{kxm1}$ *kymax $+\mathrm{kxmax})$

$+\mathrm{kfxmax}=\mathrm{kxmax}-1$, kfymax $=\mathrm{kymax}-1)$

complex psi (kxml, kyml) , den (kxml, kyml) , flowpx (kfxmax)

+ flowpy (kfymax).

+ flowpxl (kfxmax), flowpxn (kfxmax), flowpyl (kfymax), flowpyn (kfymax)

+ flowexl (kfxmax), flowexn (kfxanax), flowey l (kfymax), floweyn (kfymax).

+ flowex (kfxmax), flowey (kfymax), enflowx (kfxmax), enflowy (kfymax),

+ ennflwy (kfymax), enlflwy (kfymax), ennflwx (kfxmax), enlflwx (kfxmax)

+ eneflowx (kfxmax), eneflowy ( $k f y m a x)$, enneflwy (kfymax)

enleflwy (kfymax), enneflwx (kfamax), enleflwx (kfxmax)

real kxnorm, kynorm, ekkold (koml, kymI), le, lp, leout, lemid, lpout.

+ Ipmid, ratio2, edenold (kxml, kyml), nue, emass, dfirst, rhos

common /parms/

+ v1, gamal, gama2, epsi, le, lp, kxg, kyg, kxnorm, kynorm, kxs, kys,

+ nwrt, nwrs, $t$, dt, tol, ek, gamk, flux, nel, ratio, psio, d, dmid, dout, loc

+ init, flreal, flima, ekkold, enkold, flexold, flowex, flowey, gama3,

+ flowexl, flowexn, floweyl, floweyn, lpmid, lpout, lemid, leout, phase

+ fipxold, flowpx, flowpy, flowpxl, flowpxn, flowpyl, edenold, nue,

+ ennflwy, enlflwy, ennflwx, enlflwx, eneflowx, eneflowy, dfirst, rhos,

+ enneflwy, enleflwy, enneflwx, enleflwx, pulhgt, kxpul, kypul.

+ ratio2, kbreak

common /arrays/

+ psi, den

common /arrdim

+ nt, ntstep, pesu

External ranl

initialization c perturb psi

al $=0$.

$\mathrm{a}_{2}=0$.

$i 1=1$

$i 2=\mathrm{kxm} 2$

$j 1=$ kym2

$j 2=\mathrm{kyml}$

c for flat inition

for flat initial profile

if (init .eq. 1) then

0 do $20 \quad i=i 1, i 2$

$x k=k x n m^{*}(1-k \times n$

do $20 j=j 1, j 2$

$y k=k y n o r m^{\star}(j-k y m 2)$

if ( ( $i a b s(i-k \times m 2)$.eq. 3 and. iabs (j-kym2) .le. 3$)$.or

(iabs (j-kym2) .eq. 3 and. iabs $(1-k x m 2)$. 3)/ then

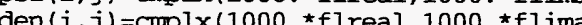

else

psi $(i, j)=c m p l x($ flreal, flima

$\operatorname{den}(i, j)=$ cmolx (flreal, flima)

if ( $i$.eq. kxm2 and. $j$.eq. kym2) goto 20

$\operatorname{psi}\left(2^{\star} \operatorname{kxm} 2-\dot{i}, 2^{\star} \operatorname{kym} 2-j\right)=\operatorname{conjg}(\operatorname{psi}(i, j))$
$\operatorname{den}\left(2^{\star} \operatorname{kxm} 2-\dot{i}, 2^{\star} \operatorname{kym} 2-j\right)=\operatorname{conjg}(\operatorname{den}(i, j))$

20

ifi (xk .eq. kxmax ${ }^{\star} k x n o r m$.and. $y k$.eq. kymax*kynorm) goto 30

$i 1=\mathrm{kxm} 2+1$

$\mathrm{i} 2=\mathrm{kxml}$

goto 10

C for regular peaked initial profile

elseif(init .eq. 2) then

11 do $21 i=i 1, i 2$

$\mathrm{xk}=\mathrm{kxnorm}{ }^{\star}(i-\mathrm{kxm} 2)$

do $21 j=j 1, j 2$

$\mathrm{yk}=\mathrm{kynorm} \star(j-\mathrm{kym} 2)$

$a k=\operatorname{sqrt}\left(x k^{\star \star} 2+y k^{\star \star} 2\right)$

al=al+psio

psi $(i, j)=$ ratio*cmpix $(a l, a l)$

$\operatorname{den}(i, j)=$ ratio* crmplx $(a 1, a 1)$

if ( $i$.eq. kxm2 and. $j$.eq. kym2) goto 21

$\operatorname{den}\left(2^{\star} \operatorname{kxm} 2-i, 2^{\star} \operatorname{kym} 2-j\right)=\operatorname{conj} j(\operatorname{den}(i, j))$

21 continue

if (xk .eq. kxmax ${ }^{\star}$ konorm and. $y k$.eq. kymax*kynorm) goto 30

$i 1=\mathrm{kxm} 2+1$

j1 =kymin

$j 1=$ kym2 +1

c for peaked initial profile with random phases

elseif (init .eq. 3) then

cfpp\$ cncall

12 do $22 \quad i=i 1, i 2$ 


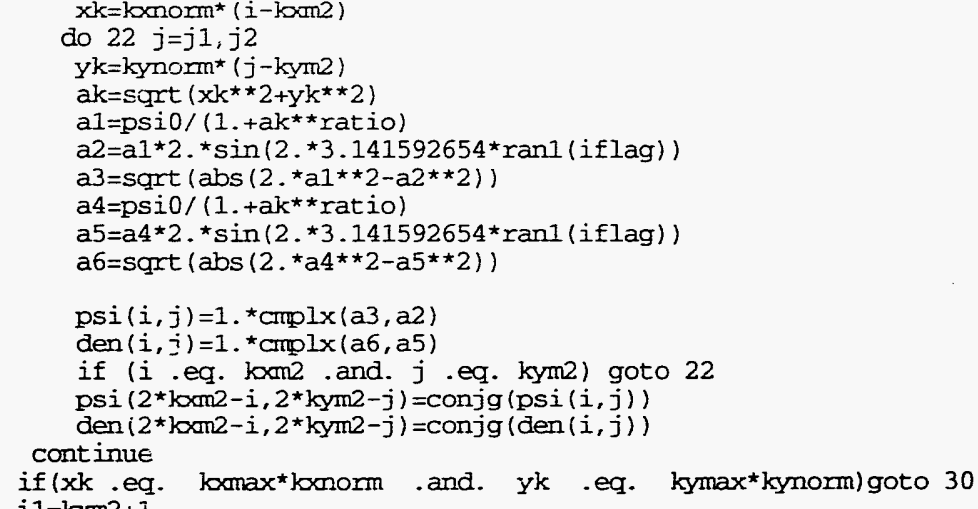

c for restart from file initspec

elseif (init eq 4) then

open (20, file =' 'initspec' ' status= ' unknown' )

\section{do $44 i=1, \mathrm{kom} 1$}

read $(20, *)(p s i(i, j), j=1, k y m-)$

44 continue

c for restart from file initspec with pulse of height pulhgt and $c$ in position kypul and kxpul (the pulse is a band)

elseif (init .eq. 5) then

open (20, file $=$ ' initspec' , status = ' unknown' )

do $45 i=1, \mathrm{kxmI}$

read $(20, \star)$ (psi $(i, j), j=1, \mathrm{kym} 1)$

45 read $(20, *)(\operatorname{den}(i, j), j=1, \mathrm{kym})$

\section{do $46 i=1, \mathrm{kxm} 1$ \\ do $46 \mathrm{j}=1$, kym}

if ( (iabs ( $i-k$ om2 $)$.eq. kopul .and. iabs (j-kym2) .le. kypul) .or.

$+(i a b s(j-k y m 2)$.eq. kypul .and. iabs $(i-k x m 2)$.le. kxpul $))$ then $\operatorname{psi}(i, j)=\operatorname{psi}(i, j) *$ pulhgt

else $j)=$ den $(i, j)$ pulhgt

$\operatorname{psi}(i, j)=p s i(i, j) \star 1$

pen $(i, j)=$ den $(i, j) \star 1$

46 c for peaked initial profile with random phases with 2 slopes elseif (init .eq. 6) then

cfpps cncall

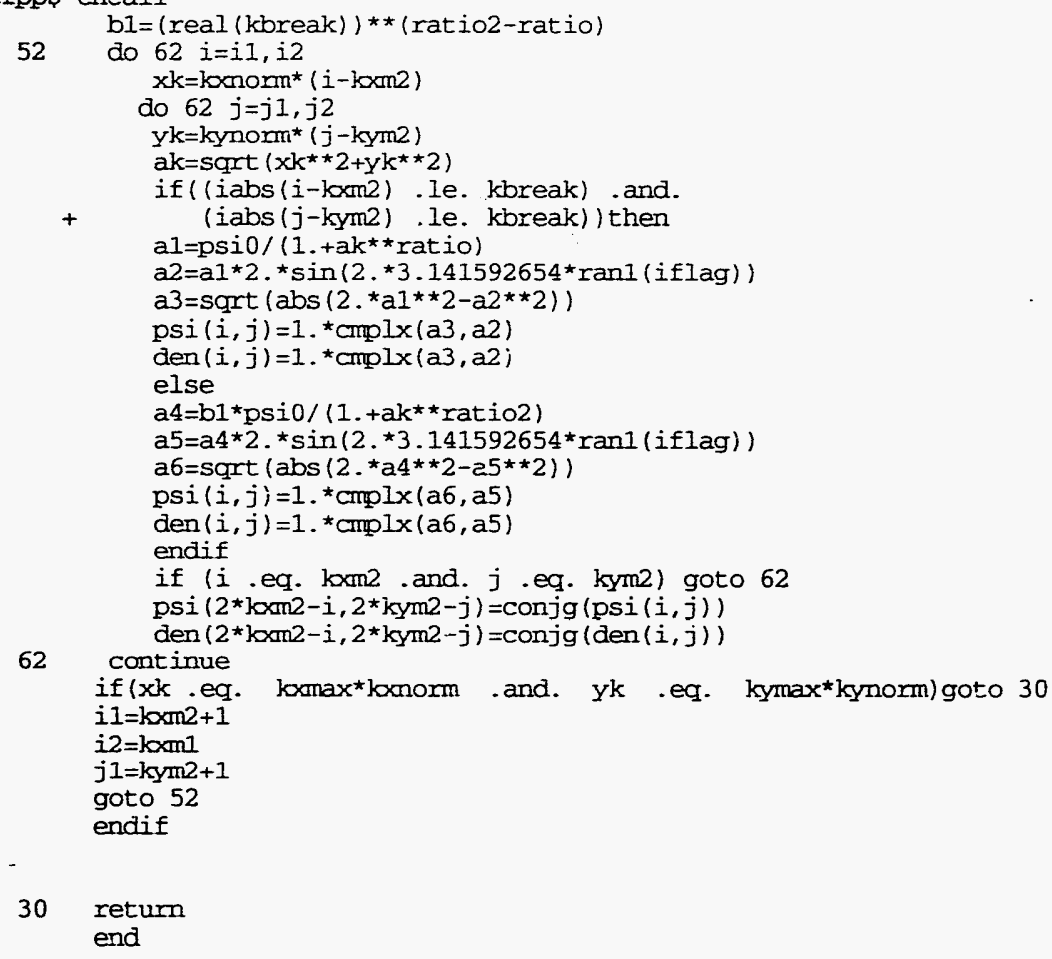

\section{C:}

function ranl (idum)

parameter $(\mathrm{ml}=259200$, ial $=7141$, icl $=54773, \mathrm{rml}=3.8580247 \mathrm{e}-6)$

parameter $(\mathrm{m} 2=134456, \mathrm{ia} 2=8121, \mathrm{ic} 2=28411, \mathrm{~mm} 2=7.4373773 \mathrm{e}-6)$ parameter (m3=243000, ia3 $=4561$, ic $3=51349$ )

data iff $10 /$

if (idum.lt.0.or.iff.eq. 0 ) then

iff $=1$

ixl=mod $(i c l-i d u m, m]$

$i x 1=\bmod (i a 1 * i x l+i c l, m i)$

ix $2=\bmod (i \times 1, m 2)$

$i x 1=\bmod (i a 1 * i \times 1+i c 1, m 1)$

$i \times 3=\bmod (i \times 1, m 3)$ 
do $11 j=1,97$

$i x 1=\bmod (i a 1 * i x 1+i c 1, m l)$

$r(i)=($ float $(i \times 1)+$ float $(i \times 2) * r m 2) * m 1$

11 idum=1

endif

$i x 1=\bmod (i a 1 * i x 1+i c 1, m 1)$

$i \times 2=\bmod (i a 2 \star i \times 2+i c 2, m 2)$

$i \times 3=\bmod (i a 3 * i \times 3+i c 3, m 3)$

$j=1+(97 * i \times 3) / m 3$

if (j.gt.97.or.j.lt.1) pause

$\operatorname{ran} 1=r(j)$

$r(j)=($ float $(i x 1)+$ float $(i \times 2) * m 2) \star m m 1$

return
erid



subroutine psilin (anl , u,v, kces, kyy)

parameter (komax $=10$, kymax $=10, \mathrm{kxml}=2 \star \mathrm{k}$ max $+1, \mathrm{kym} 1=2 * \mathrm{kymax}+1$,

$+\mathrm{kxm} 2=\mathrm{kxmax}+1, \mathrm{kym} 2=\mathrm{kymax}+1, \mathrm{nn}=4$ * $(\mathrm{kom} 1 * \mathrm{kymax}+\mathrm{kxmax})$

$+\mathrm{kfxmax}=\mathrm{kxmax}-1, \mathrm{kfymax}=\mathrm{kymax}-1)$

complex psi (koml, kyml), den (koml , kyml), flowpx (kfxmax).

flowpy (kfymax),

flowpxl (kfxmax), flowpxn (kfxmax), flowpyl (kfymax), flowpyn (kfymax),

+ flowexl ( $k f(x \max$ ), flowexn (kfxmax), floweyl (kfymax), floweyn (kfymax),

(

+ eneflowx (kfxmax), eneflowy (kfymax), enneflwy (kfymax),

real kxnorm, kynorm, ekkold (kxml, kyml), le, lp, leout, lemid, lpout,

+ lpmid, ratio2, edenold ( $k \times m l, k y m I)$, nue, emass, dfirst, rhos

common /parms/

+ v1, gamal, gama2, epsi, le, lp, kxg, kyg, kxnorm, kynorm, kxs, kys,

+ nwrt, nwrs, $t$, dt, tol, ek, gamk, flux, ncl, ratio,psio, d, dmid, dout, loc

+ init,flreal,flima, ekkold, emkold, flexold, flowex, flowey, gama3,

+ flowexl, flowexn, floweyl, floweyn, lpmid, lpout, lemid, leout, phase,

+ flpxold, flowpx, flowpy, flowpxl, flowpxn, flowpyl, edenold, nue,

+ amp, flowpyn, en, gamen, ed, et, ekold, enold, enflowx, enflowy, emass,
+ ennflwy, enlflwy, ennflwx, enlflwx, eneflowx, eneflowy, dfirst, rhos,

+ enneflwy, enleflwy, enneflwx, enleflwx

common /arrays/

+ psi,den

common /arrdim/

+ nt, ntstep, pesu

complex $\mathrm{u}$, ic, anl, $\mathrm{v}$

c initialization

ic $=(0 ., 1$.

$\mathrm{xk}=\mathrm{k} \times \mathrm{nnorm} \mathrm{m}^{\star} \mathrm{kox}$

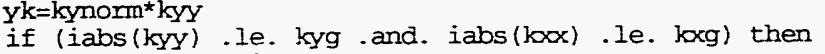

c construct linear portion

gl=gamal

$d 1=d f$ irst ${ }^{\star}$ nue



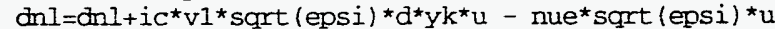

c elseif(iabs (kyy) .gt. kys .or. iabs (kxx) .gt. kxs)then c construct linear portion

$\mathrm{gl}=$ gama 3

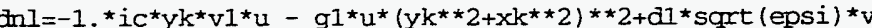

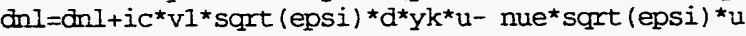

else

c construct linear portion

$\mathrm{g} 1=\mathrm{gama} 2$

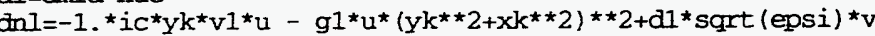

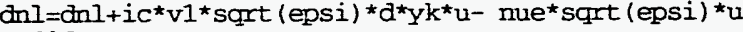
endif

return

end

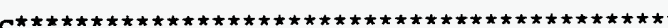

subroutine fpsi (kx,ky,u,v,psikp)

pameter (lomax $=10, \mathrm{kymax}=10, \mathrm{k} \times \mathrm{km} \mathrm{I}=2$ * $\mathrm{k} \times \mathrm{max}+1, \mathrm{kyml}=2$ *kymax +1

$+\mathrm{kxm} 2=\mathrm{kx} \max +1, \mathrm{kym} 2=\mathrm{kymax}+1, \mathrm{nn}=4^{*}\left(\mathrm{kxm} \mathrm{I}^{*} \mathrm{kymax}+\mathrm{kxmax}\right)$,

+ kfxmax $=k x m a x-1, k f y$ max $=k y$ max -1 )

complex psi (koml, kyml), den (kxml, kyml), flowpx (kfxmax) ,

+ flowpy (kfymax),

+ flowpxl (kfxmax), flowpxn (kfxmax), flowpyl (kfymax) , flowpyn (kfymax)

+ ilowexl (kfxmax), flowexn (kfxmax), floweyl (kfymax), floweyn (kfymax),

+ flowex (kfxmax), flowey (kfymax), enflowx (kfxmax), enflowy (kfymax),

+ eneflowx (kfxmax), eneflowy (kfymax), enneflwy (kfymax).

+ enleflwy (kfymax), enneflwx (kfxmax), enleflwx (kfxmax)

real komorm, kynorm, ekkold ( $\mathrm{koml}$, kyml), le, lp, leout, lemid, lpout.

+ lpmid, ratio2, edenold (kxm?, kyml), nue, emass, dfirst, rhos

cormon /parms/

+ v1, gama1, gama2, epsi, le, Ip, kxg, kyg, kxnorm, kynorm, kxs, kys,

+ nwrt, nwrs, $t$, dt, tol, ek, gamk, flux, ncl, ratio, psio, d, dmid, dout, loc

+ init,flreal, flima, ekkold, enkold, flexold, flowex; flowey, gama3,

+ flowexl, flowexn, floweyl, floweyn, Ipmid, Ipout, lemid, leout, phase

+ flpxold, flowpx, flowpy, flowpxl, flowpxn, flowpyl, edenold, nue,

+ amp, flowpyn, en, gamen, ed, et, ekold, enold, enflowx, enflowy, emass,

+ ennflwy, enlflwy, ennflwx, enlflwx, eneflowx, eneflowy, dfirst, rhos,

+ enneflwy, enleflwy, enneflwx, enleflwx

conmon /arrays/

+ psi, den

cormon /arrdim/

+ nt, ntstep, pesu

complex $u$ (koml, kyml) , v (kxml, kyml) , psikp, dn, dnl, pin, din

$+\operatorname{dn} 1, \operatorname{dn} 2, i c$

ic $=(0 ., 1$.

$x k=k \times n o r m * k 0 x$

$y k=$ kynorm $\star k y$

pin $=u(k x+k x m 2, k y+k y m 2)$ 
din $=v(k x+b \mathrm{kxm} 2, k y+k, y m 2)$



call psilinidnl, pin, din, bx, ky)

call conv2 $u, u, k x, k y$, an 2 )

psikp=dnl $-10^{\star} d^{\star} .5^{\star} d n$

psikp=psikp/ $((1 .-\operatorname{sqrt}(\operatorname{epsi})) \star a k 1)$

return

end



parameter (kxmax $=10, \mathrm{kymax}=10, \mathrm{kxml}=2 * \mathrm{kxmax}+1, \mathrm{kym}=2$ *kymax +1 ,

$+\mathrm{kxm} 2=\mathrm{kxmax}+1, \mathrm{kym} 2=\mathrm{kymax}+1, \mathrm{nn}=4$ * $\{\mathrm{kxm} 1 *$ kymax + kxmax $)$,

+ kfxmax $=$ komax -1, kfymax $=$ kymax -1$)$

complex psi (kaml, kyml), den (kxml, kyml) , flowpx (kfxmax)

+ flowpy (kfymax)

+ flowpxl (kfxmax), flowpxn (kfxmax), flowpyl (kfymax), flowpyn (kfymax),

+ flowexl (kfxmax), flowexn (kfxmax), floweyl (kfymax), floweyn (kfymax),

+ flowex (kfxmax), flowey (kfymax), enflowx ( $k f x \max$ ), enflowy (kfymax),

+ ennflwy (kfymax), enlflwy (kfymax), ennflwx (kfxmax), enlflwx (kfxmax),

+ eneflowx ( $\mathrm{kfxmax}$ ), eneflowy (kfymax), enneflwy (kfymax),

+ enleflwy (kfymax), enneflwx (kfxmax), enleflwx (kfxmax)

real bxnorm, kynorm, ekkold ( $k x m l, k \mathrm{kml})$, le, lp, leout, lem

+ lpmid, ratio2, edenold (koml, kyml), nue, emass, df irst, rhos

comon /parms/

+ v1, gamal, gama2, epsi, le, lp, kxg, kyg, loxnorm, kynorm, kxs, kys.

+ nwrt, nwrs, $t, d t$, tol, ek, gamk, flux, ncl, ratio, psio, d, dnid, dout, loc,

+ init,flreal, fl ima, ekkold, emkold, flexold, flowex, flowey, gama3,

+ flowexl, flowexn, floweyl, floweyn, lpmid, lpout, lemid, leout, phase,
+ flpxold, flowpx, flowpy, flowpxl, flowpxn, flowpyl, edenold, nue,

+ amp, flowpyn, en, gamen, ed, et, ekold, enold, enflowx, enflowy, emass,

+ emnflwy, enlflwy, ennflwx, enlflwx, eneflowx, eneflowy, dfirst, rhos,

+ ennflwy, enlflwy, ennflwx, enlflwx, eneflo

common /arrays/

+ psi,den

common /arrdim

common /arrdim

complex u (koml, kyml) , v (kxml, kyml), pin, din, psikp, cinl, dnl, ic

c initialization

$i c=(0 ., 1$.
$y k=k y n o m m^{*}$

$\mathrm{pin}=\mathrm{u}(\mathrm{kx}+\mathrm{kxm} 2, \mathrm{ky}+\mathrm{krym} 2)$

din $=v(k x+k x m 2, k y+k y m 2)$

c construct linear portion.

$d n l=\left(-1 .{ }^{\star} i c^{\star} y k^{\star} d I^{\star} v 1{ }^{\star} p i n+n u e^{\star} p i n-n u e^{\star} d i n\right) /$ emass

call conv (u, v, bx, by, dn 1 )

dnl=dni-le* $d^{\star} \cdot 5^{*} \operatorname{dn} 1$ returr.

end

subroutine fenl (neq, $x, y$, Yprime)

parameter $(\mathrm{kxmax}=10, \mathrm{kymax}=10, \mathrm{koml}=2 \star \mathrm{k} x \mathrm{max}+1, \mathrm{kyml}=2$ *kymax +1

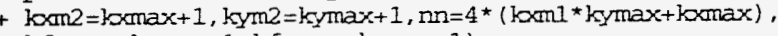

$+\mathrm{kfxmax}=\mathrm{kxmax}-1, \mathrm{kfymax}=\mathrm{kymax}-1)$

complex psi (koml, kyml), den (banl, kyml), flowpx (kfxmax),

+ flowpy (kfymax), flowpxn (kfxmax), flowpyl (kfymax), flowpyn (kfymax),

+ flowexl (kfxmax), flowexn (kfxmax), flowey l(kfymax), floweyn (kfymax)

+ flowex (kfxmax), flowey (kfymax), enflowx (kfxmax), enflowy (kfymax),

+ ennflwy (kfymax), enlflwy (kfymax), ennflwx (kfxmax), enlflwx (kfxmax),

+ eneflowx (kfxmax), eneflowy (kfymax), enneflwy (kfymax),

+ enleflwy (kfymax), enneflwx (kfxmax), enleflwx (kfxmax)

real kxnorm, kynorm, ekkold ( $\mathrm{kmml}$, kyml), le, lp, leout, lemid

+ lpmid, ratio2, edenold (koml, kyml), nue, emass, dfirst, rhos
common /parms/
+ vl, gamal, gama2, epsi, le, lp, kxg, kyg, konom, kynorm, kxs, kys,

+ vl, gamal, gama2, epsi, le, lp, kxg, kyg, konom, kynorm, kxs, kys,

+ nwrt, nwrs, t, dt, tol, ek, gamt, flux, ncl, ratio, psio, d, dmid, dout, loc,
+ init, flreal, flima, ekkold, emkold, flexold, flowex, flowey, gama3,

+ init, flreal, flima, ekkold, emkold, flexold, flowex, flowey, gama,

+ flpxold, flowpx, flowpy, flowpxl, flowpxn, flowpyl, edenoid, nue,

+ flpxold, flowpx, flowpy, flowpxl, flowpxn, enflow, enflowy, emass,

+ amp, flowpyn, en, gamen, ec, et, ekold, enold, enflowx, enflowy, emass,

+ enneflwy, enleflwy, enneflwx, enleflwx

common /arrays/

psi,den

common /arrdim/

+ nt,ntstep, pesu

complex psikp, nko, u (kxml, kyml), denkp, v (kxml, kyml)

+ pin, din

c initialization

kount $=0$

kount $1=k \times m I * k y$ max + kcmax

kount $d=2^{\star}\left(\right.$ born $1^{\star} k$ kymax + kxmax $)$

kount $\mathrm{dl}=3 *(\mathrm{kxml} * \mathrm{kymax}+\mathrm{kxmax})$

c convert $y$ to psi, den

c get yprim

$i 1=1$
$i 2=k x \max$
$j 1=1$

$j 2=$ kym2

do $20 i=i 1$, i2

$i k=i-k 0 m 2$

do $20 j=j 1, j 2$

$j k=j-1$

kount $=$ kount +1

$c$ get $\mathrm{dn} / \mathrm{dt}=\mathrm{fpsi}$

pin $=u(i k+k x m 2, j k+k y m 2)$ 
din $=v(i k+k x m 2, j k+k y m 2)$

call tpsi (ik, jk, u, v,psikp)

call fden (ik, $j k, u, v$, denkp)

yprime (kount) =real (psikp)

yprime (kount tkount1)=ainag (psikp)

yprime (kount $+k$ ountdi) $=a$ inag (den)

20 continue

if (ik .eq. kxmax .and. $j k$.eq. kymax) goto 30

il $=\mathrm{k} \times \mathrm{m} 2$

$i 2=\mathrm{k} \times \mathrm{m} 2$

$j 1=2$

c

30 return

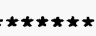

subroutine $\mathrm{fcn} 2$ (ned, $x, y, \mathrm{ml}, \mathrm{mu}, \mathrm{pd}, \mathrm{nrpd}$ )

parameter $(\mathrm{kxmax}=10, \mathrm{~kg}$ max $=10, \mathrm{kxml}=2 \star \mathrm{lom} \max +1, \mathrm{kyml}=2 \star \mathrm{kymax}+1$

$+\mathrm{kxm} 2=\mathrm{kxmax}+1, \mathrm{kym} 2=\mathrm{kymax}+1, \mathrm{mn}=4$ * $(\mathrm{kxm} 1 * k y m a x+k x m a x)$

+ kfxmay:=kxmax $-1, \mathrm{kfy}$ max $=k y$ max -1$)$

complex psi (kxml, kyml), den (kxml, kyml), flowpx (kfxmax),

+ flowpy (kfymax)

+ flowpxl (kfxmax), flowpxn (kfxmax), flowpyl (kfymax) , flowpyn (kfymax),

+ flowexl (kfxmax), flowexn (kfxmax), floweyl (kfymax), floweyn (kfymax),

+ flowex (kfxmax), flowey (kfymax), enflowx (kfxmax), enflowy (kfymax),

+ ennflwy (kfymax), enlflwy (kfymax), ennflwx (kfxmax), enlflwx (kfxmax),

+ eneflowx (kfxmax), eneflowy (kfymax), enneflwy (kfymax),

real kxmorm, kynorm, ekkoid (koml, kyml), le, lp, leout, lemid, lpout.

+ lpmid, ratio2, edenold (kxml, kyml), nue, emass, dfirst, rhos

common /parms/

+ vl, gamal, gama2, epsi, le, lp, loxg, kyg, loxnorm, kynorm, loxs, kys,

+ nwrt, nwrs, t, dt, tol, ek, gamk, flux, ncl, ratio, psio, d, dmid, dout, loc

+ init, flreal, flima, ekkold, enkold, flexold, flowex, flowey, gama3,

+ flowexl, flowexn, floweyl, floweyn, Ipmid, lpout, lemid, leout, phase

+ flpxold, flowpx, flowpy, flowpxl, flowpxn, flowpyl, edenold, nue,

+ amp, flowpyn, en, gamen, ed, et, ekold, enold, enflowx, enflowy, emass,

+ ennflwy, enlflwy, ennflwx, enlflwx, eneflowx, eneflowy, dfirst, rhos,

+ enneflwy, enleflwy, enneflwx, enleflwx

cormon /arrays/

+ psi,den

common /arräim/

+ nt,ntstep, pesu

integer $\mathrm{mm}$

real $x, y(n n), p d(n x p d, n n), q$

c the jacobian

complex $u(k x m 1, k y m 1), v(k x m 1, k y m 1)$

kount $=0$

kountm=kxm1 *kymax+komax

kount $=2 *($ kxm 1 *kymax + kxmax $)$

countam $=3$ * $(\mathrm{kxm} 1$ *kymax $+\mathrm{k}$ oxmax $)$

lount $=0$

lountm $=\mathrm{kx} \times \mathrm{m} I$ *kymax $+\mathrm{k}$ xemax

lount $=2$ * (kom 1 *kymax +loxmax $)$

lountan=3* (kaml*kymax+kxmax)

$a b=1 . /(1 .-\operatorname{sqrt}($ epsi) $)$

call invert $(y, u, v)$

c process

$\mathrm{mI}=1$

$\mathrm{m} 2=\mathrm{kxmax}$

$\mathrm{nI}=1$

$2=k y m 2$

i $1=1$

$i 2=$ lomax

$j 1=1$

55

do $60 \mathrm{~m}=\mathrm{mI}, \mathrm{m} 2$

$k x=(m-10 \pi(2) * k \times n o r n$

do $50 \mathrm{n}=\mathrm{n} 1, \mathrm{n} 2$

$\mathrm{k}=(\mathrm{n}-1) \star \mathrm{km}$

ky $=(n-1)$

lount $=$ lount +1

$\mathrm{kxl}=(i-\mathrm{kom} 2) \star \mathrm{kmorm}$

do $20 j=j 1, j 2$

$\mathrm{kyl}=(j-1) * \mathrm{kynorm}$

kount=kount +1

kxarr $=\mathrm{m}$

kyarr $=\mathrm{n}+$ kymax

kxlarr $=1$

kylarr $=j+k y$ max

kxxarr=bxarr-bxlarr

$a b l=a b /\left(1 .+\left(k x^{\star \star 2+k y} y^{\star \star 2}\right) \star r_{h o s} \star \star 2\right)$

if (iabs (ky) .le. kyg and. iabs (kx) .le. $k x g$ ) then

$\mathrm{g}=$ gamal $=$ dfirst ${ }^{\star}$ nue

elseif (iabs (ky) gt. kys .or. iabs (kx) .gt. kxs) then $\mathrm{g}=$ gama $3 *(\mathrm{ky} *$ (

else

$g=\operatorname{gama2}{ }^{\star}\left(k y^{\star \star} 2+k x^{\star \star 2}\right) \star \star 2$

endif

$\mathrm{akl}=\mathrm{k} \times 1 \star \star 2+\mathrm{ky} 1 \star \star 2$

$a k=(k x-k x l) \star \star 2+(k y-k y l) \star \star 2$

if ( $(m$.eq. i) .and. (n .eq. j)) then

pd (lount, kount $)=\left(-1 .{ }^{*} g-\right.$ nue $\operatorname{sqrt}($ epsi $\left.)\right) \star a b l$

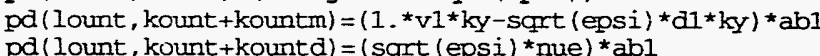


pd (lount, kount + kount $d m)=0$

$\operatorname{pd}($ lount + lount d, kount $)=(-1, * \mathrm{v} 1 * \mathrm{ky}+\mathrm{sqrt}($ epsi) $* \mathrm{dl} * \mathrm{ky}) * a b 1$ pd (lount+lountd, kount + kountm $)=\left(-1 .{ }^{*} g-\right.$ nue ${ }^{\star}$ sqrt (epsi) $) * a b$ pd (lount +lountd, kount + kount $)=0$.

pd $($ lount + lountd, kount + kountdm $)=($ sqrt $($ epsi $) *$ nue $) * a b 1$

pa (lount +lountm, kount $)=1 .{ }^{*}$ nue

pa (lount+lountm, kount +koun $(m)=1$. * $\mathrm{vl}{ }^{*} \mathrm{~d}{ }^{*} \mathrm{ky} /$ emass

pd(lount + lountm, kount + kounta) $=-1 . *$ nue/emas

$\operatorname{pd}($ lount + lount $d m$, kount $)=-1 . \star v 1 * d \star k y /$ emass

pd (lount + lountam, kount + kountm) $=1$.

作

\section{else}

if ((kxoarr .le. 0) .or. (kxoarr .gt. kxml) .or
(kyyarr.le. 0) or. (kyyarr gt. kyml)) then

pd (lount, kount $)=0$

pd (lount, kount + kountm) $=0$

pd (lount, kount + kount $)=0$.

pd (lount, kount + kount $d m)=0$

pd (lount +lountm, kount $)=0$.

pd (lount+lountm, kount + kountm $)=0$.

pd(lount + lountrn, kount + kount $d)=0$.

pd (lount + lountm, kount + kount $\mathrm{c} m)=0$

pd (lount + lountd, kount $)=0$.

pd (lount+lountd, kount + kountdm) $=0$.

pd (lount+lountom, kount $)=0$.

pd (lount + lount dm, kount + kountm) $=0$.

pd (lount + lounted, kount + kounted $)=0$.

else

pd (lount, kount) $=($

$++10^{\star} d^{\star} \cdot 5^{\star}\left(k x^{\star} k y-k y I^{*} k x\right) * r e a l(u(k 0 x a r r, k y y a r r))$

$+*(a k l-a k)) * a b l$

pd (lount, kount + kountm $)=($

$+10^{\star} \mathrm{d}^{\star} .5^{\star}\left(\mathrm{kx} 1^{\star} \mathrm{ky}-\mathrm{ky} 1^{\star} \mathrm{kx}\right)$ *aimag (u (kxxarr, kyyarr) $)$

$+\quad *(a k l-a k)) * a b I$

pa (lount, kount + kount $d)=0$

pd (lount, kount + kountdm) $=0$ pd (lount+lountm, kount $)=($

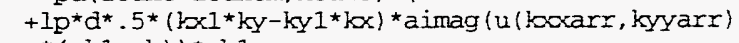

$\star(a k l-a k)) * a b l$

pd (lount + lountm, kount + kountm $)=1$

$+1 p^{\star} d^{\star} \cdot 5^{\star}\left(k x I^{*} k y-k y 1^{*} k x\right)$ * real (u (boxarr, kyyarr)

*(akl-ak))*abl

pd (lount +lountm, kount + kount $d)=0$.

pd (lount+lountm, kount + kount $d m$ ) $=0$.

pd (lount+lountd, kount $)=\left(-1 .{ }^{\star} l e^{\star} d^{\star} .5^{\star}(k \times 1 * k y-k y 1 * k x)\right.$

*real (v (boxarr, kyyarr) )*2.)

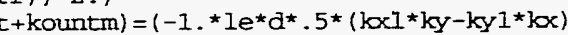

*aimag $(v($ koxarr, kyyarr $)) \star 2$.)

(1)

* real (u (loorar , leyarr) $\star 2$

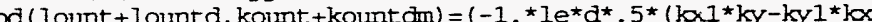

*aimag (u (kxxarr, kyyarr) )*2.)

$p d($ lount + lountdm, kount $)=\left(-1 .{ }^{*} l e^{\star} d^{\star} \cdot 5 *(k x l * k y-k y l \star k x)\right.$

*aimag (v (kocxarr, kyyarr) )*2.

pd (lount+lount dre, kount + kountm $)=\left(-1 .{ }^{\star} i e^{\star} d^{\star} .5^{\star}\left(k x l^{\star} k y-k y l^{\star} k x\right)\right.$

*real ( $\mathrm{v}(\mathrm{kxc}$ arr, kyyarr $)) * 2$.)

pd (lount+lountam, kount+kountd) $=\left(-1 .{ }^{\star} l e^{\star} \mathrm{d}^{\star} \cdot 5^{\star}\left(\mathrm{kxl} \mathrm{l}^{\star} \mathrm{ky}-\mathrm{ky} 1^{\star} \mathrm{kx}\right)\right.$

*aimag (u (boxarr, kyyarr))*2.)

pd (lount+lountdm, kount $+\mathrm{kount} d \mathrm{~m})=\left(-1 .{ }^{*} \mathrm{e}^{\star} \mathrm{d}^{\star} .5 *\left(\mathrm{kxl}{ }^{*} \mathrm{ky}-\mathrm{ky} 1 * \mathrm{kor}\right)\right.$

$+\quad$ *real (u (koxarr, kyyarr) )*2.)

endif

continue

continue

.and. (kylarr .eq. kyml)) goto 35

$i 1=\mathrm{kxm} 2$

$2=\mathrm{kom}$

goto 25

35 if ((kxarr .eq. kxml) and. (kyarr .eq. kyml)) goto 70

iI $=1$ =kmax

$j 1=1$

50 continue

continue

$m 2=k m$

$n]=2$

goto 55

70 return 


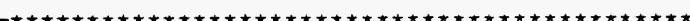

subrout ine timead

parameter $(\mathrm{kxmax}=10, \mathrm{kymax}=10, \mathrm{kcml}=2 \star \mathrm{kxmax}+1, \mathrm{kyml}=2 \star k y \max +1$

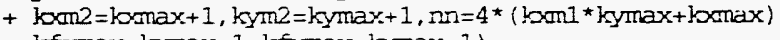

, flowpx (kfxmax),

+ flowpy (kfymax)

f flowpxn (kfxmax), flowpyl (kfymax), flowpyn (kfymax),

flower (kfxmax), floweyl (kfymax), floweyn (kfymax)

+ ennflwy (kfymax) enlflwy (kfymax), ennflwx (kfxmaxi, enlflwx (kfxmax)

+ eneflowx (kfxmax), eneflowy (kfymax), enneflwy (kfymax),

real kxnorm, kynorm, ekkold (kcml, kyml), le, Ip, leout, lemid, lpout.

+ lpmid, ratio2, edenold (koml, kyml), nue, emass, dfirst, rhos

common /parms/

+ v1, gamal, gama2, epsi, le, lp, kxg, kyg , kxnorm, kynorm, loss, kys

+ nwrt, nwrs, t, dt, tol, ek, gamk, flux, ncl, ratio, psio, d, dnid, dout, loc

+ init,flreal, flima, ekkold, emkold, flexold, flowex, flowey, gama3,

+ flowexl, flowexn, floweyl, floweyn, lpmid, lpout, lemid, leout, phase,

+ flpxold, flowpx, flowpy, flowpxl, flowpxn, flowpyl, edenold, nue,

+ amp, flowpyn, en, gamen, ed, et, ekold, enold, enflowx, enflowy, emass,

+ emeflwy, enleflwy, enneflwx, enleflwx

comon /arrays/

psi.den

common /arrdim/

nt, ntstep, pesu

real $y(n n), t f$, rwork $(22+9 * n n+n n * \star 2)$

integer iwork $(20+n n), l i w, l r w$

$c$ intialization$$
\text { liw }=n+20
$$

$1 r w=22+9 * n n+n n \star \star 2$

if $(t$.eq. 0.0$)$ then

itask $=1$

istate 10

iopt $=0$

itol $=1$

tola $=$ tol *amp

endif

$h=d t / 100$

$t f=t+d t$

c convert psi to $y$

$$
\text { call convert (psi, den, } y \text { ) }
$$

c timestep

call lsode ( $f \mathrm{cnl}, \mathrm{nn}, \mathrm{y}, t, t f$, itol, tol, tola, itask, istate, iopt, rwork,

+ Irw, iwork, liw, fen $2, \mathrm{mf}$ )

$c$ check for problems

o) call endit (istate, rwork:(11)

write $(9,60)$ iwork (11), iwork (12), iwork (13)

write $(9, *)$ ' istate $=$ ", istate c convert y back to psi

$$
\text { call invert (y,psi, den) }
$$

format $i / 12 h$ no. steps $=, i 4$, IIh no. $f-s=, i 4$

+ lih no. j-s $=, i 4$ i

rets

$C^{\star \star \star \star \star \star *}$

subroutine conv (u, v, kxo, kyy, w)

parameter $(\mathrm{kxmax}=10, \mathrm{kymax}=10, \mathrm{kxml}=2+\mathrm{k}$ max $+1, \mathrm{kyml}=2 \star \mathrm{k} k \mathrm{mmax}+1$

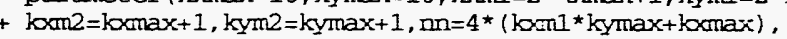

$+\mathrm{kfxmax}=\mathrm{kxmax}-1, \mathrm{kfymax}=\mathrm{ky}$ max -1

complex psi (kxml, kyml), den (kxmi, : vyml) , flowpx (kfxmax)

+ flowpy (kfymax)

+ flowpxI (kfxmax), flowpxn (kfxmax), flowpyl (kfymax), flowpyn (kfymax),

+ flowexl (kfxmax), flowexn (kfxmax), floweyl (kfymax), floweyn (kfymax)

+ flowex (kfxmax), flowey (kfymax), enflowx (kfxmax), enflowy (kfymax),

+ ennflwy (kfymax), enlflwy (kfymax), ennflwx (kfxmax), enlflwx (kfxmax)

+ eneflowx (kfxmax), eneflowy (kfymax), enneflwy (kfymax)

+ enleflwy (kfymax), enneflwx (kfxmax), enleflwx(kfxmax)

real konorm, kynorm, ekkola (kxml, kyml), le, lp, leout, lemid, lpout.

+ lpmid, ratiu2, edenold ( $\mathrm{kxml}$, kyml), nue, emass, dfirst, rhos

common /parms/

+ v1, gamal, gama2, epsi, 1e, lp, kxg, kyg, lonorm, kynorm, kxs, kys,

+ nwrt, nwrs, t, dt, tol, ek, gank, flux, ncl, ratio, psio, d, dnid, dout, loc

+ init, flreal, flima, ekkold, enkold, flexold, flowex, flowey, gama,

+ flowexl, flowexn, floweyl, floweyn, lpmid, lpout, lemid, leout, phase.

+ flpxold, flowpx, flowpy, flowpxl, flowpxn, flowpyl, edenold, nue,

+ amp, flowpyn, en, gamen, ed, et, ekold, enold, enflowx, enflowy, emass,

+ emflwy, enlflwy, ennflwx, enlflwx, eneflowx, eneflowy, dfirst, rhos.

+ enneflwy, enleflwy, enneflwx, enleflwx

common /arrays/

psi,den

common / arrdim

comolex u(koml, kyml) , v (kxml, kyml) , w, wl

$c$ intialization

c process

$w=10$
$i 1=1$

$\mathrm{i} 2=\mathrm{k} \times \mathrm{mm} 1+\mathrm{k} \times \mathrm{x}$

if $(\mathrm{k} x \mathrm{x} \cdot \mathrm{gt}$

0) then

il $=\mathrm{k} \infty \mathrm{x}+\mathrm{x}$

$i 2=\operatorname{loxml}$

$$
\begin{aligned}
& \text { do } 20 i=i 1, i 2 \\
& \text { do } 20 \quad j=k y y+1, k y m 1 \\
& k x 1=i-k x m 2 \\
& k y 1=j-k y m 2 \\
& k x m o d=k 0 x-k x 1 \\
& k y m o d=k y y-k y 1 \\
& \text { bxm=kxm2 }+k x m o d \\
& k y m=k y m 2+k y m o d
\end{aligned}
$$


c convolute

$w 1=(u(i, j) * v($ lom, $k y m)-u(k 0 m, k y m) * v(i, j))$

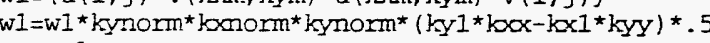

20 continue

return

end

\section{C}

subrout ine conv2 ( $\mathrm{u}, \mathrm{v}, \mathrm{k}, \mathrm{x}, \mathrm{kyy}, \mathrm{w})$

parameter ( $\mathrm{kxmax}=10, \mathrm{kymax}=10, \mathrm{kxml}=2{ }^{*} \mathrm{kxmax}+1, \mathrm{kyml}=2$ * $\mathrm{kymax}+1$

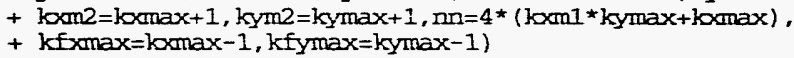

complex psi (kxml, kyml), den (kxml, kyml), flowpx (kfxmax),

+ flowpy (kfymax)

+ flowpxl (kfumax), fllowpxn (kfxmax), flowpyl (kfymax), flowpyn (kfymax)

+ flowexl (kfxmax), flowexn (kfxmax), floweyl (kfymax), floweyn (kfymax),

+ flowex (kfxmax), flowey (kfymax), enflowx (kfxmax), enflowy (kfymax),

+ ennflwy (kfymax), enlflwy (kfymax), ennflwx (kfxmax), enlflwx (kfxmax)

+ eneflowx (kfxmax), eneflowy (kfymax), enneflwy (kfymax)

+ enleflwy (kfymax), enneflwx (kfxmax), enleflwx (kfxmax)

+ lpmid, ratio2, edenold ( $\mathrm{kml}$, kyml), nue, emass, dfirst, rhos

common /parms/

+ v1, gamal, gama2, epsi, le, lp, loxg, kyg, kxnorm, kynorm, kxs, kys,

+ nwri, nwrs, $t$, dt, tol, ek, gamk, flux, ncl, ratio,psio, d, dnid, dout, loc,

+ init, flreal, fl ima, ekkold, emkold, flexold, flowex, flowey, gama3,

+ flowexl, flowexn, floweyl, floweyn, lpmid, lpout, lemid, leout, phase

+ flpxold, flowpx, flowpy, flowpxl, flowpxn, flowpyl, edenold, nue,

+ amp, flowpyn, en, gamen, ed, et, ekold, enold, enflowx, enflowy, emass,

+ enneflwy, enleflwy, enneflwx, enleflwx

common /arrays

+ psi,den

common /arrdim/

nt, ntstep, pesu

$c$ intialization

c process

$w=(0,0,0.0)$
$i 1=1$
$i 2=\operatorname{kxm} I++\infty 0 x$

i2 $=k x m I+k 0 x$
if $(k x x . g t .0)$ then

il $=\mathrm{k} 0 \mathrm{x} x+1$

i2 $=\operatorname{lom} 1$

$$
\begin{aligned}
& \text { do } 20 i=i 1, i 2 \\
& \text { do } 20 j=k y y+1, k y m 1 \\
& k \times 1=i-k x m 2 \\
& k y 1=j-k y m 2 \\
& k x m o d=k x x-k \times 1 \\
& k y m o d=k y y-k y 1
\end{aligned}
$$

$k x m=b x m 2+$ lexmod

$k y m=k y m 2+k y m o d$

$\mathrm{ak} 1=\mathrm{k} \times 1 \star \star 2+\mathrm{ky} 1 * \star 2$

ak $=\mathrm{komod}^{\star \star} 2+\mathrm{kyym} \mathrm{d}^{\star \star 2}$

c convolute

$w I=(u(i, j) * *$ (tomorm*kynorm

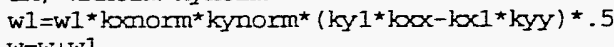

continue

end

subroutine invert $(\mathrm{y}, \mathrm{u}, \mathrm{v})$

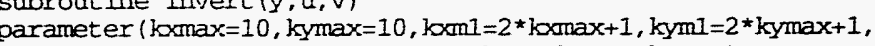

$+\operatorname{kxm} 2=\mathrm{kxmax}+1, \mathrm{kym} 2=\mathrm{kymax}+1, \mathrm{nn}=4 *(\mathrm{kxml} * \mathrm{kymax}+\mathrm{kxmax})$

kfxmax=kamax-1, kfymax-kymax-1)

complex psi (kxml, kyml), den (koml, kyml), flowpx (kfxmax)

+ flowpy (kfymax),

+ flowpxl (kfxmax), flowpxn (kfxmax), flowpyl (kfymax), flowpyn (kfymax),

+ flowexl (kfxmax), flowexn (kfxmax), floweyl (kfymax), floweyn (kfymax)

+ Hlowex (kfxmax), flowey (kfymax), enflowx (kxmax), enflowy (kfymax),

+ eneflowx (kfxmaxi, eneflowy (kfymax), enneflwy (kfymax),

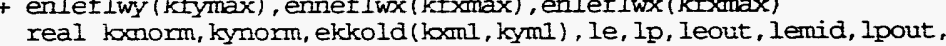

lpmid, ratio2, edenold (baml, kyml), nue, enass, dfirst, rhos

$\begin{aligned} & \text { common / parms/ } \\ + & \text { v1, gamal, gama2, epsi. le, lp, kog, kyg, bonorm, kynorm, kxs, kys, }\end{aligned}$

+ nwrt, nwrs, $t$, dt, tol, ek, gamk, flux, ncl, ratio, psio, d, dmid, dout, loc,

+ init,flreal, flima, ekkold, enkold, flexold, flowex, flowey, gama3,

+ flowexl, flowexn, floweyl, floweyn, Ipmid, lpout, lemid, Ieout, phase,

+ Elpxold, flowpx, flowpy, flowpxl, flowpxn, flowpyl, edenold, nue,

+ amp, flowpyn, en, gamen, ed, et, ekold, enold, enflowx, enflowy, emass,

+ ennflwy, enlflwy, ennflwx, enlflwx, eneflowx, eneflowy, dfirst, rhos,

+ enneflwy, enleflwy, ennefiwx, enleflwx

common /arrays/

+ psi,den

common /arrdim

nt, ntstep, pesu

complex $\mathrm{u}(\mathrm{kxm}, \mathrm{kyml}), \mathrm{ul}, \mathrm{v}(\mathrm{kxml}, \mathrm{kyml})$, v2 real $y(n n)$

c initialization

kount $=0$

kountm $=\mathrm{kxm} 1$ *kymax $+\mathrm{k}$ oxmax

kount $=2 *(\mathrm{kxdl}$ *kymax + kxmax $)$

c process

$$
\text { i1 }=1
$$


$j 1=1$

10 do $20 \quad i=i 1, i 2$

kxarr $=i$

do $20 j=j 1, j 2$

kyarr $=$ kymax $+j$

$\mathrm{ul}=\mathrm{cmpl} \mathrm{x}$ ( $\mathrm{y}$ (kount), $\mathrm{y}$ (kount + kountm)

$\mathrm{u}($ kxarr, kyarr $)=\mathrm{u}]$

v $2=$ cmplx $(y$ (kount + kount $d), y$ (kount + kount $d m)$

$\mathrm{v}$ (kxarr, kyarr) $=\mathrm{v} 2$

\section{$i x=\operatorname{lom} 1-i+1$}

$u(i x, j x)=$ conjg $(u 1)$

20

$v(i x, j x)=$ conjg $(v 2)$

continue

if (kxarr .eq. kxml .and. kyarr .eq. kyml)goto 30

$i 1=\mathrm{k} x \mathrm{~m} 2$

$j 1=2$

goto 10

c

$$
\text { retu }
$$



subroutine convert $(u, v, y)$

parameter (kxmax =10, kymax $=10, \mathrm{kxml}=2$ *komax $+1, \mathrm{kyml}=2$ *kymax +1

$+\mathrm{kom} 2=\mathrm{kom} \max +1, \mathrm{kym} 2=\mathrm{kymax}+1, \mathrm{nn}=4 *(\mathrm{kom} 1 * \mathrm{kymax}+\mathrm{kxmax})$,

$+\mathrm{kfxmax}=\mathrm{kxmax}-1, \mathrm{kfymax}=\mathrm{ky}$ max -1$)$

complex psi (kxml, kyml), den (kxml, kyml), flowpx (kfxmax),

flowpxl (kfxmax), flowpxn (kfxmax), flowpyl (kfymax), flowpyn (kfymax),

flowexl (kfxmax), flowexn (kfxmax), flowey l (kfymax), floweyn (kfymax),

+ flowex (kfxmax), flowey (kfymax), enflowx (kfxmax), enflowy (kfymax).

+ ennflwy (kfymax), enlflwy (kfymax), ennflwx (kfxmax), enlflwx (kfxmax)

+ eneflowx (kfxmax), eneflowy (kfymax), enneflwy (kfymax),

real kxnorm, kynorm, ekkold (koml, kyml), le, lp, leout, lemid, lpout,

+ Ipmid, ratio2, edenold ( $\mathrm{koml}$, kyml) , nue, emass, dfirst, rhos

common /parms/

+ v1, gamal, gama2, epsi, le, lp, kxg, kyg, konom, kynorm, kxs, kys,

+ nwrt, nwrs, $t$, dt, tol, ek, gamk, flux, ncl, ratio, psio, d, dmid, dout, loc

+ init, flreal, flima, ekkold, enkold, flexold, flowex, flowey, gama3.

+ flowexl, flowexn, floweyl, floweyn, lpmid, lpout, lemid, leout, phase,

+ flpxold, flowpx, flowpy, flowpxl, flowpxn, flowpyl, edenold, nue,

+ amp, flowpyn, en, gamen, ed, et, ekold, enold, enflowx, enflowy, emass.

+ ennflwy, enlflwy, ennflwx, enlflwx, eneflowx, eneflowy, dfirst, rhos,

+ enneflwy, enlefl'vy, enneflwx, enleflwx

common /arrays/

+ psi, den

comon /arrdim

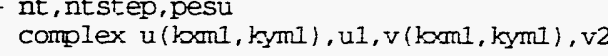

real $y(n n$

C initialization

kount $=0$

kountm $=$ bomI 1 kymax + loxmax

kouncd $=2 * 1$ (bent:

c process

i2 $=$ kxmax

$i 2=k x m a x$
$j 1=1$

10

do $20 i=i 1, i 2$

oxarr=

do $20 j=j 1, j 2$

kyarr $=$ kymax $+j$

$y$ (kount) $=$ real (u (kxarr, kyarr) )

(kount +kountm) =aimag ( $\mathrm{u}$ (kxarr, kyarr $)$

$y($ kount + kountd $)=$ real $(v($ kxarr, kyarr $))$
$y($ kount+kountdm) $=$ aimag (v (kxarr, kyarr $))$

20

continue

if (kxarr .eq. kxml .and. kyarr .eq. kyml) goto 30

il $=\mathrm{k} \times \mathrm{m} 2$

$\mathrm{i} 2=\mathrm{kxm}$

$j 1=2$

goto 10

c

return

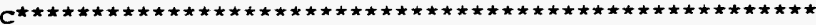

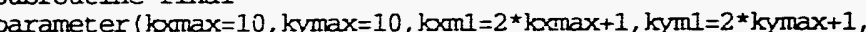

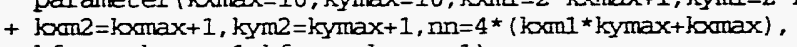

kf

complex psi (kxml, kyml), den (kxml, kyml), flowpx (kfxmax),

+ flowpy (kfymax)

+ flowpxl (kfxmax), flowpxn (kfxmax), flowpyl (kfymax), flowpyn (kfymax)

+ flowexl (kfxmax), flowexn (kfxmax), floweyl (kfymax), floweyn (kfymax)

+ flowex (kfxmax), flowey (kfymax), enflowx (kfxmax), enflowy (kfyax),

+ eneflowx ( $k f x m a x)$, eneflowy (kfymax), enneflwy (kfymax)

+ enleflwy (kfymax), enneflwx (kfxmax), enleflwx (kfxmax)

real bxnorm, kynorm, ekkold ( $\mathrm{kmm}$, kyml), le, Ip, leout, lenud, Ipout.

+ lpmid, ratio2, edenold ( kaml, kyml) , nue, emass, dfirst, rhos

common /parms/

+ v1, gama1, gama2, epsi, le, lp, kxg, kyg, kxnorm, kynorm, kxs, kys,

+ nwrt, nwrs, $t$, dt, tol, ek, gamk, flux, ncl, ratio, psio, d, dmid, dout, loc

+ init, flreal, flima, ekkold, emkold, flexold, flowex, flowey, gama3,

+ flowexl, flowexn, floweyl, floweyn, lpmid, lpout, lemid, leout, phase,

+ flpxold, flowpx, flowpy, flowpxl, flowpxn, flowpyl, edenold, nue.

+ amp, flowpyn, en, gamen, ed, et, ekold, enold, enflowx, enflowy, emass 
+ ennflwy, enlflwy, ennflwx, enlflwx, eneflowx, eneflowy, dfirst, rhos, + enneflwy, enleflwy, enneflwx, enleflwx

coninon /arrays

psi,den

common /arrdim

write $(6,100)$

write $(10,100)$

100 format (4x,' das ende')

return

end

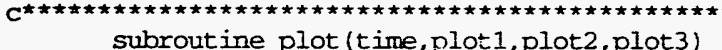

parameter $(\mathrm{kxmax}=10, \mathrm{kymax}=10, \mathrm{kxml}=2 \star \mathrm{kxmax}+1, \mathrm{kyml}=2$ *kymax +1 ,

$+\operatorname{loxn2} 2=\mathrm{kxmax}+1, \mathrm{kym} 2=\mathrm{kymax}+1, \mathrm{nn}=4$ * $(\mathrm{kxml}$ *kymax+komax),

$+\mathrm{kf}$ xmax $=\mathrm{kxmax}-1, \mathrm{kfy}$ max $=\mathrm{ky}$ max -1 )

complex psi (koml, kyml), den (koml, kyml), flowpx (kfxmax) ,

+ flowpy (kfymax)

+ flowpxl (kfxmax), flowpxn (kfxmax), flowpyl (kfymax), flowpyn (kfymax)

+ flowexl (kfxmax), flowexn (kfxmax), floweyl (kfymax), floweyn (kfymax)

+ flowex (kfxmax), flowey (kfymax), enflowx (kfomax), enflowy (kf

+ ennflwy (kfymax), enlflwy (kfymax), ennflwx (kffmax), enlflwx (kfxmax)

+ eneflowx (kfxmax), eneflowy (kfymax), enneflwy (kfymax)

real kxnorm, kynom, ekkold (kxml, kyml), le, lp, leout, lemid, lpout,

Ipmid, ratio2, edenold (kxml, kyml), mue, emass, dfirst, rhos

common /parms/

+ v1, gama1, gama2, epsi, le, lp, kxg, kyg, kxnorm, kynorm, kxs, kys,

+ mwrt, nwrs, $t$, dt, tol, ek, gamk, flux, ncl, ratio, psio, d, dmid, dout, loc

+ init,flreal, flima, ekkold, enkold, flexold, flowex, flowey, gama3.

+ flowexl, flowexn, floweyl, floweyn, lpmid, lpout, lemid, leout, phase.

+ flpxold, flowpx, flowpy, flowpxl, flowpxn, flowpyl, edenold, nue,

+ ennflwy, enlflwy, ennflwx, enlflwx, eneflowx, eneflowy, dfirst, shos.

+ enneflwy, enleflwy, enneflwx, enleflwx

common /arrays/

psi,den

common /arrdim

+ nt, ntstep, pesu

real time (4000), plot $1(4000)$, plot $2(4000)$
do $30 i=1$, ntstep
$\operatorname{ploty}(i, 1)=\operatorname{plot} 1(i)$
$\operatorname{ploty}(i, 2)=\operatorname{plot} 2(i)$

$\operatorname{ploty}(i, 3)=\operatorname{plot} 3(i)$

continue

c replacing the old vax plotting progam with nmfecc's quikdraw routines call opngks

call exmxy (t ime, ploty, 4000, 3,4000,

c + 'energy vs times' 


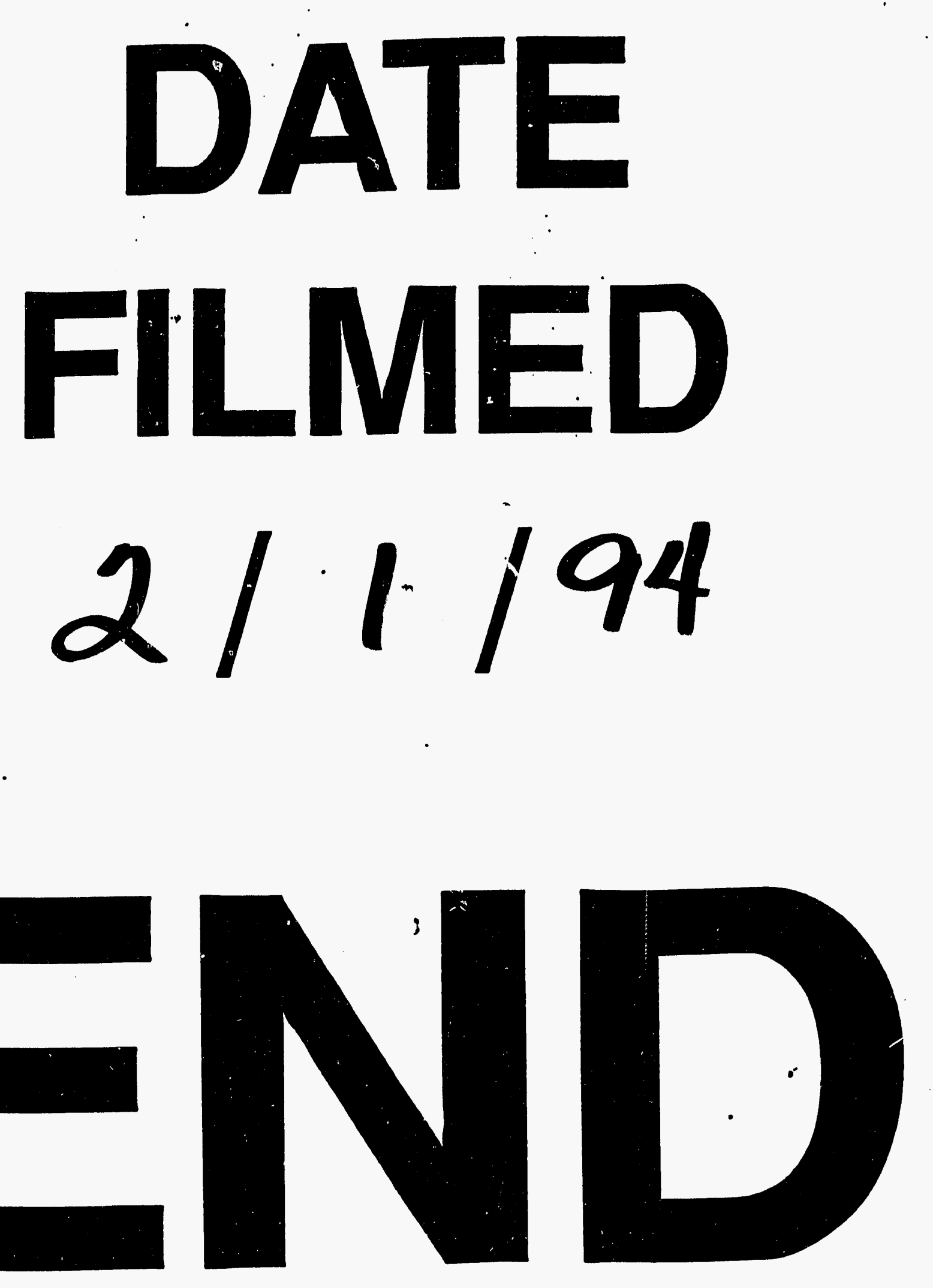


